Supplementary Information

\title{
Metallaphotoredox Perfluoroalkylation of Organobromides
}

\author{
Xiangbo Zhao and David W. C. MacMillan* \\ Merck Center for Catalysis at Princeton University, Princeton, New Jersey 08544, USA
}

${ }^{*}$ Corresponding author.Email: dmacmill@princeton.edu 


\section{Table of Contents}

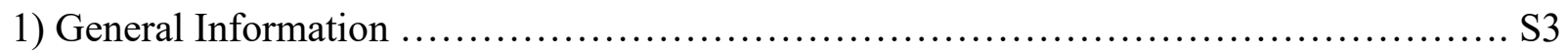

2) Synthesis and Characterization of Related Materials...................................... S4

3) Reaction Optimization and Control Experiments.................................... 7

4) General Procedures and Experimental Data..............................................

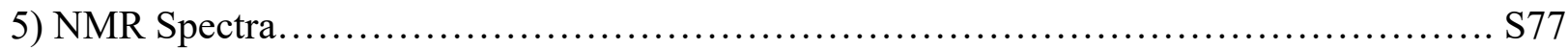

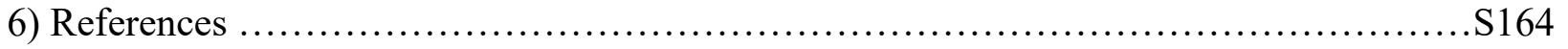




\section{1) General information}

Commercial reagents were used without prior purification unless otherwise indicated. All organic reaction solvents were purified according to the method of Grubbs. ${ }^{1}$ Unless otherwise indicated, filtration of heterogeneous mixtures was performed using ChemRus $20 \mathrm{~mL}$ or $60 \mathrm{~mL}$ disposable filters. Organic solutions were concentrated under reduced pressure on a Büchi rotary evaporator using a water bath. Chromatographic purification of products was accomplished on an automated Biotage Isolera ${ }^{\mathrm{TM}}$ Spektra System with Silicycle SiliaSep ${ }^{\mathrm{TM}}$ cartridges (or SiliaSep ${ }^{\mathrm{TM}} \mathrm{C} 18$ cartridges in the case of reverse phase chromatography). Thin-layer chromatography (TLC) was performed on Analtech Uniplate $0.25 \mathrm{~mm}$ or Supelco $0.20 \mathrm{~mm}$ silica gel F-254 plates. Visualization of the developed chromatogram was performed by fluorescence quenching, $\mathrm{KMnO}_{4}$ stain, or iodine stain. ${ }^{1} \mathrm{H}$ and ${ }^{13} \mathrm{C}$ NMR spectra were recorded on a Bruker Avance III NMR 500 $\mathrm{MHz}$ instrument or a Bruker NanoBay Avance III HD NMR $400 \mathrm{MHz}$ instrument, ${ }^{19} \mathrm{~F}$ NMR was recorded on NanoBay 300 (282 MHz) or Varian Inova (376 MHz) spectrometers, and are internally referenced to the residual proteo-solvent signals (note: $\mathrm{CDCl}_{3}$ referenced at $7.26 \mathrm{ppm}$ and 77.16 ppm, respectively; DMSO-d 6 referenced at 2.50 and $39.52 \mathrm{ppm}$, respectively). Data for ${ }^{1} \mathrm{H}$ are reported as follows: chemical shift $(\delta \mathrm{ppm})$, multiplicity $(\mathrm{s}=$ singlet, $\mathrm{d}=$ doublet, $\mathrm{t}=$ triplet, $\mathrm{q}=$ quartet, $\mathrm{p}=$ pentet, $\mathrm{h}=$ hextet, hept $=$ heptet, $\mathrm{m}=$ multiplet, $\mathrm{br}=$ broad $)$, coupling constant $(\mathrm{Hz})$, and integration. Data for ${ }^{13} \mathrm{C}$ NMR are reported in terms of chemical shift; multiplicity and coupling constants are included only in the case of coupling with ${ }^{19} \mathrm{~F}$ nuclei. IR spectra were recorded on a Perkin Elmer Spectrum 100 FTIR spectrometer and are reported in wavenumbers $\left(\mathrm{cm}^{-1}\right)$. High Resolution Mass Spectra (HRMS) were obtained from the Princeton University Mass Spectral Facility. Gas chromatography (GC) was performed on an Agilent 6850 Series chromatograph with split less capillary injection and FID detection. 


\section{2) Synthesis and characterization of photocatalysts and aminosilane reagents}

(a) The photocatalysts $\operatorname{Ir}\left[(\mathrm{F}-\mathrm{mppy})_{2}(\mathrm{phen})\right]\left(\mathrm{PF}_{6}\right)(\mathbf{1}),{ }^{2} \operatorname{Ir}\left[(\mathrm{ppy})_{2}(\mathrm{bpy})\right]\left(\mathrm{PF}_{6}\right)(\mathbf{1 5}),{ }^{3}$ were prepared according to literature procedures.

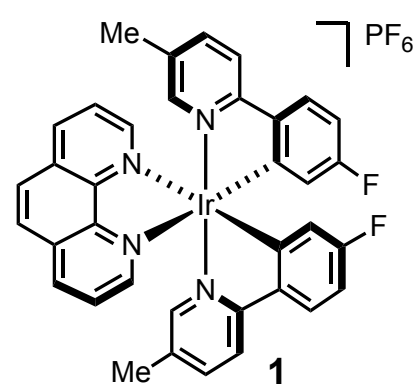

$\operatorname{Ir}\left[(\text { F-mppy })_{2}(\right.$ phen $\left.)\right] \mathrm{PF}_{6}$<smiles></smiles>

$\operatorname{Ir}\left[(\text { ppy })_{2}(\mathrm{bpy})\right] \mathrm{PF}_{6}$

(b) The silane reagent AdNHSi(TMS) 3 (4) was prepared according to a literature procedure. ${ }^{4}$

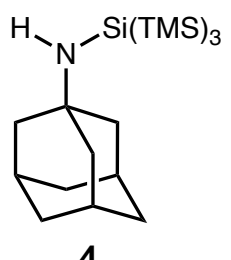

Cyclic Voltammogram: $E_{\mathrm{pa}}=+0.66 \mathrm{~V}$ (vs Standard calomel electrode (SCE) in MeCN).

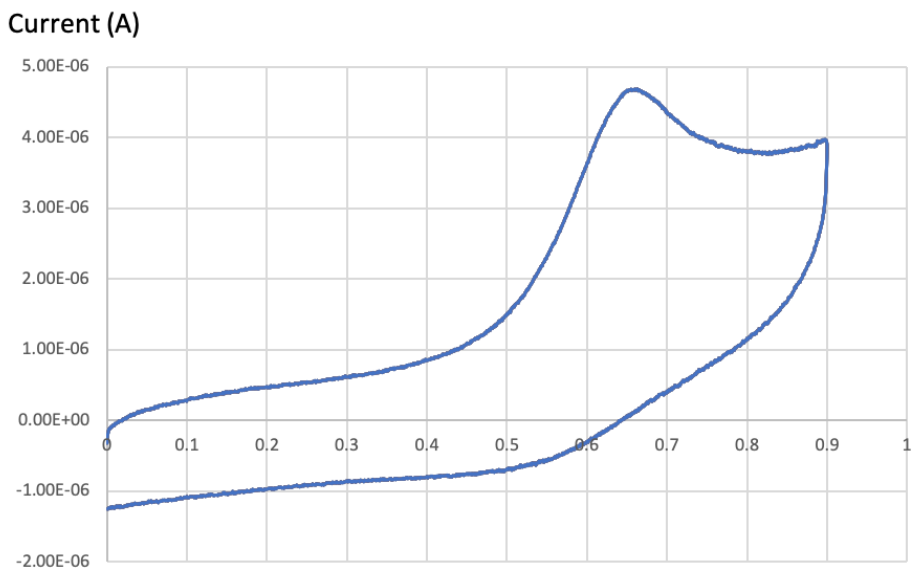

Potential (V vs SCE)

Figure S1. Cyclic voltammogram of $\mathrm{AdNHSi}(\mathrm{TMS})_{3}$ (4) in $\mathrm{MeCN}$. 
(c) Preparation of (hetero)aryl bromides

The following compounds were prepared according to literature procedures: 4-bromo-N-(2,6dimethoxypyrimidin-4-yl)-N-methylbenzenesulfonamide, ${ }^{5}$ methyl 2-(1-(4-bromobenzoyl)-5methoxy-2-methyl-1H-indol-3-yl)acetate, ${ }^{5}$ and $(2 R, 3 R, 4 R, 5 R)$-2-(acetoxymethyl)-5-(6-bromo$9 H$-purin-9-yl)- tetrahydrofuran-3,4-diyl diacetate. ${ }^{6}$

The syntheses of previously unreported aryl bromides are included below.

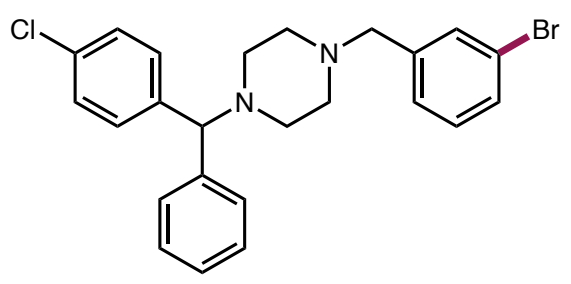

\section{1-(3-bromobenzyl)-4-((4-chlorophenyl)(phenyl)methyl)piperazine (S1)}

To a $50 \mathrm{~mL}$ round bottom flask equipped with a stir bar was added sodium borohydride $(0.38 \mathrm{~g}$, 10 mmol, 2 equiv). The flask was capped and the headspace sparged with $\mathrm{N}_{2}$, then chloroform (16 $\mathrm{mL})$ was added. The solution was stirred and cooled to $0{ }^{\circ} \mathrm{C}$ in an ice bath, and acetic acid $(2.6 \mathrm{~mL}$, 46 mmol, 9.2 equiv) was added dropwise over 10 minutes, producing $\mathrm{H}_{2}$ gas. After bubbling ceased, a solution of 1-((4-chlorophenyl)(phenyl)methyl)piperazine (1.43 g, $5.0 \mathrm{mmol}, 1$ equiv) in chloroform $(4 \mathrm{~mL})$ was added, followed by 3-bromobenzaldehyde $(0.615 \mathrm{~mL}, 5.25 \mathrm{mmol}, 1.05$ equiv). The reaction then became homogenous and turned light yellow. The reaction was allowed to proceed for 16 hours at room temperature.

The reaction mixture was alkalized to $\mathrm{pH} 8$ with saturated $\mathrm{K}_{2} \mathrm{CO}_{3}(8 \mathrm{~mL})$. The mixture was transferred to a separatory funnel containing water $(50 \mathrm{~mL})$ and EtOAc $(50 \mathrm{~mL})$. The aqueous layer was extracted with EtOAc $(3 \times 50 \mathrm{~mL})$. The combined organics were washed with brine, dried over $\mathrm{MgSO}_{4}$, filtered, and concentrated in vacuo affording a thick yellow oil. Purification by flash column chromatography $(0-7 \%$ EtOAc/hexanes) provided the title compound as a thick yellow oil (1.56 g, $3.5 \mathrm{mmol}$, 70\% yield). 
${ }^{1}$ H NMR (500 MHz, CDCl $) \delta 7.49(\mathrm{~s}, 1 \mathrm{H}), 7.41-7.35(\mathrm{~m}, 5 \mathrm{H}), 7.35-7.10(\mathrm{~m}, 7 \mathrm{H}), 4.23(\mathrm{~s}$, $1 \mathrm{H}), 3.49(\mathrm{~s}, 2 \mathrm{H}), 2.63-2.27(\mathrm{~m}, 8 \mathrm{H})$.

${ }^{13}$ C NMR (125 MHz, CDCl $\left.\mathbf{3}\right) \delta 142.24,141.45,140.65,132.59,132.10,130.23,129.85,129.30$, $128.72,128.67,127.95,127.81,127.23,122.50,75.52,62.41,53.34,51.84$.

IR (film) $v_{\max } 3027,2933,2808,2765,1670,1594,1569,1487,1452,1336,1298,1135,1088$, $1069,1007,852,802,757,718,697,671 \mathrm{~cm}^{-1}$.

HRMS (GC-EI-TOF) $\mathrm{m} / z$ calcd. for $\mathrm{C}_{24} \mathrm{H}_{25} \mathrm{BrClN}_{2}\left([\mathrm{M}+\mathrm{H}]^{+}\right) 455.0884$, found 455.0878 . 


\section{3) Reaction Optimization and Control Experiments}

Photocatalyst stock solution: Prepared fresh before each screen by dissolving photocatalyst (5.0 $\mu \mathrm{mol}$ ) in $10 \mathrm{~mL}$ of anhydrous $\mathrm{MeCN}$. This solution was sonicated for 5 minutes before use.

General Procedures: To an oven-dried 8-mL vial equipped with a magnetic stir bar was added 4bromobenzonitrile ( $18.2 \mathrm{mg}, 0.1 \mathrm{mmol}, 1.0$ equiv.), base ( $0.4 \mathrm{mmol}, 4.0$ equiv.), and silane reagent (0.18 mmol, 1.8 equiv.), copper source ( $0.02 \mathrm{mmol}, 0.2$ equiv.), and ligand ( $0.025 \mathrm{mmol}, 0.25$ equiv). To this reaction vial was added photocatalyst stock solution ( $1.0 \mathrm{~mL}, 0.5 \mu \mathrm{mol}, 0.5 \mathrm{~mol} \%)$ via syringe, followed by $\mathrm{TMSR}_{\mathrm{F}}\left(\mathrm{R}_{\mathrm{F}}=\mathrm{CF}_{3}, \mathrm{C}_{2} \mathrm{~F}_{5}, \mathrm{C}_{3} \mathrm{~F}_{7}\right)(0.3 \mathrm{mmol}, 3.0$ equiv.). After the reaction mixture was stirred for 10 min, the vial septum was pierced by an $18 \mathrm{G}$ needle, which was left in the septum for the duration of the reaction to allow for air flow without solvent evaporation. The reaction vial was then irradiated with two $34 \mathrm{~W}$ Kessil H150-Blue (maximum blue with maximum intensity setting) from $6 \mathrm{~cm}$ away with fan cooling or placed in the Integrated Photoreactor. ${ }^{7}$ Internal standard 1,4-difluorobenzene (10.2 $\mu \mathrm{L}, 0.1 \mathrm{mmol}, 1.0$ equiv.) was added and an aliquot was taken for ${ }^{1} \mathrm{H}$ NMR and ${ }^{19} \mathrm{~F}$ NMR analysis in DMSO- $d_{6}$. Formation of the desired product was confirmed by comparing the NMR \& GC-MS data with sample of authentic product.

\section{Typical Reaction Set-Up}

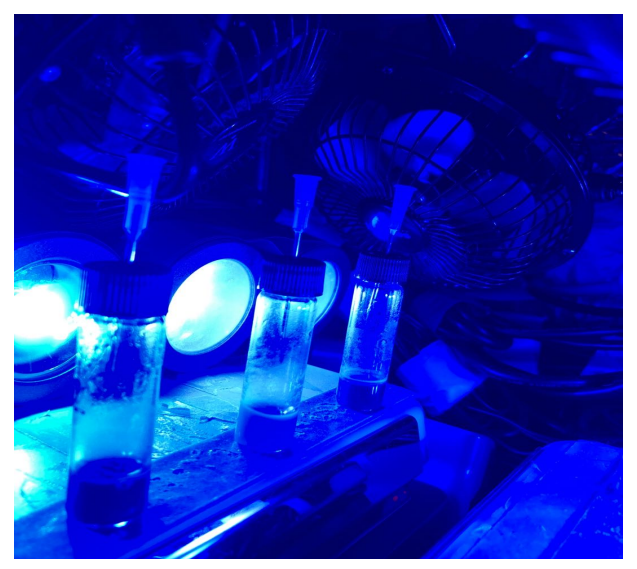

Kessil lights set-up

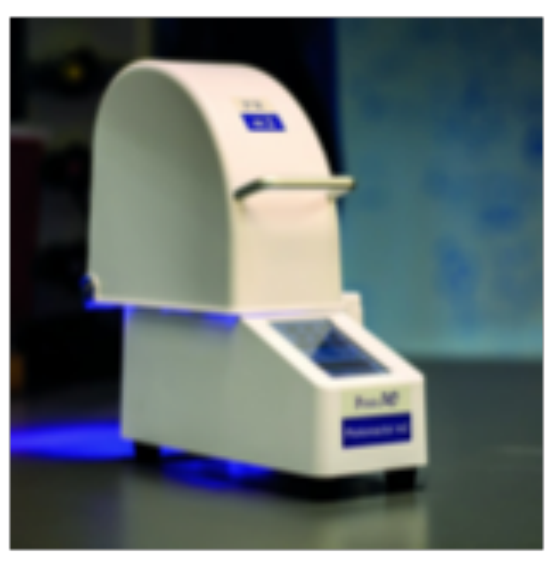

Merck Integrated photoreactor 
(a) Optimization tables of trifluoromethylation of aryl bromides

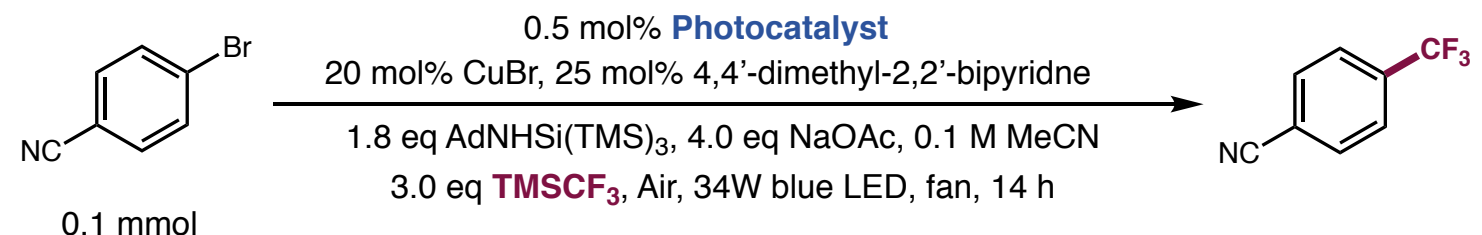<smiles>CC(C)C(C)(C)C(C)(C)C</smiles>

$\operatorname{Ir}(\mathrm{ppy})_{2}(\mathrm{bpy}) \mathrm{PF}_{6}$

$64 \%$ yield

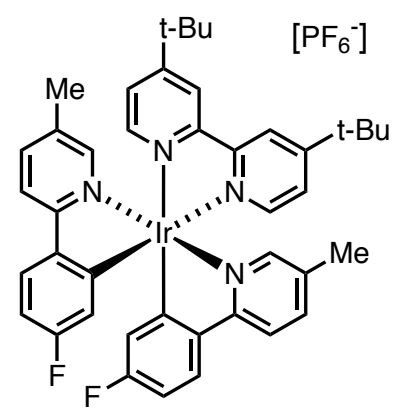

$\operatorname{Ir}(\mathrm{F}-\mathrm{mppy})_{2}(\mathrm{dtbbpy}) \mathrm{PF}_{6}$

$78 \%$ yield<smiles>CC(C(C)(C)C)C(C)(C)C</smiles>

$\operatorname{Ir}(\text { ppy })_{2}(\mathrm{dtbbpy}) \mathrm{PF}_{6}$

$66 \%$ yield

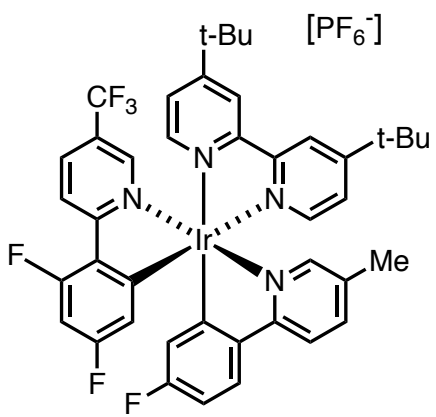

$\operatorname{Ir}\left(\mathrm{dF}\left(\mathrm{CF}_{3}\right) \mathrm{ppy}\right)_{2}(\mathrm{dtbbpy}) \mathrm{PF}_{6}$ $65 \%$ yield

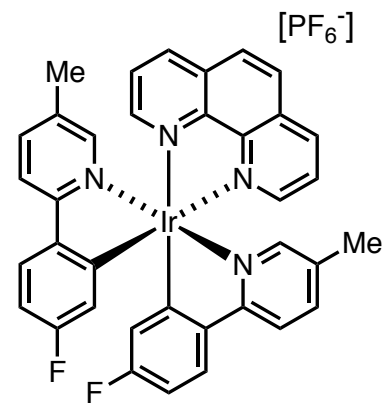

$\operatorname{Ir}(\mathrm{F}-\mathrm{mppy})_{2}($ phen $) \mathrm{PF}_{6}$

$87 \%$ yield

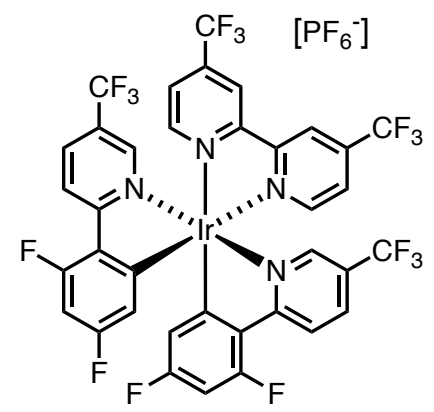

$\operatorname{Ir}\left(\mathrm{dF}\left(\mathrm{CF}_{3}\right) \mathrm{ppy}\right)_{2}\left(\mathrm{dCF}_{3} \mathrm{bpy}\right) \mathrm{PF}_{6}$ $70 \%$ yield

Figure S2. Evaluation of different photocatalysts. Yields determined by ${ }^{1} \mathrm{H}$ NMR and ${ }^{19} \mathrm{~F}$ NMR. 


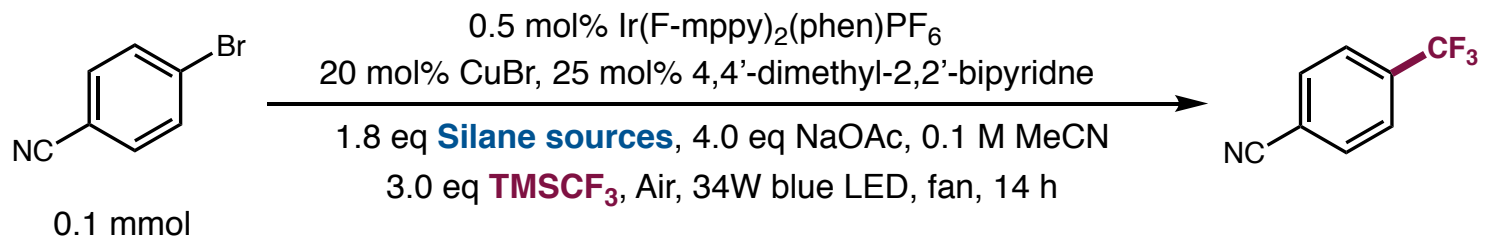

\begin{tabular}{cc} 
Silane & Yield \\
\hline $\mathrm{TMS}_{3} \mathrm{SiH}$ & $15 \%$ \\
$\mathrm{TMS}_{3} \mathrm{SiOH}$ & $35 \%$ \\
$t$-BuNHSi(TMS) & $85 \%$ \\
AdNHSi $(\mathrm{TMS})_{3}$ & $87 \%$
\end{tabular}

Figure S3. Evaluation of different silane reagents. Yields determined by ${ }^{19} \mathrm{~F}$ NMR.

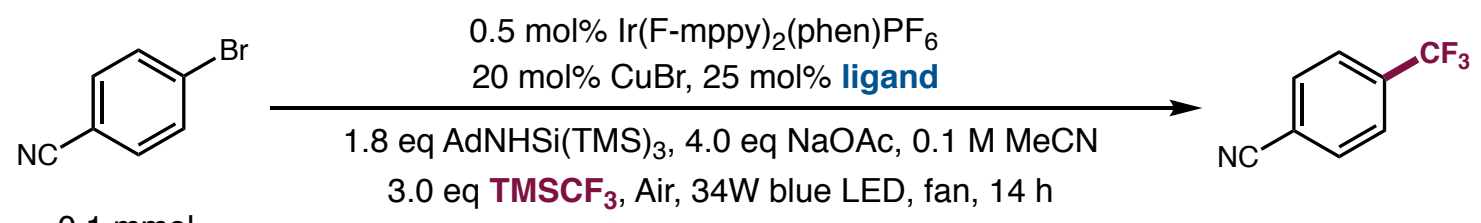

$0.1 \mathrm{mmol}$

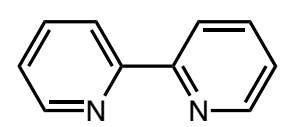

$78 \%$ yield<smiles>CC(=O)c1ccnc(-c2cc(C(C)=O)ccn2)c1</smiles>

$10 \%$ yield<smiles>Cc1ccnc(-c2cc(C)ccn2)c1</smiles>

$87 \%$ yield<smiles>c1cnc2c(c1)ccc1cccnc12</smiles>

$58 \%$ yield<smiles>CC(C)(C)c1ccnc(-c2cc(C(C)(C)C)ccn2)c1</smiles>

$82 \%$ yield<smiles>Cc1ccnc2c1ccc1c(C)ccnc12</smiles>

$50 \%$ yield<smiles>COc1ccnc(-c2cc(OC)ccn2)c1</smiles>

$58 \%$ yield

$48 \%$ yield

Figure S4. Evaluation of different ligands. Yields determined by ${ }^{19} \mathrm{~F}$ NMR. 


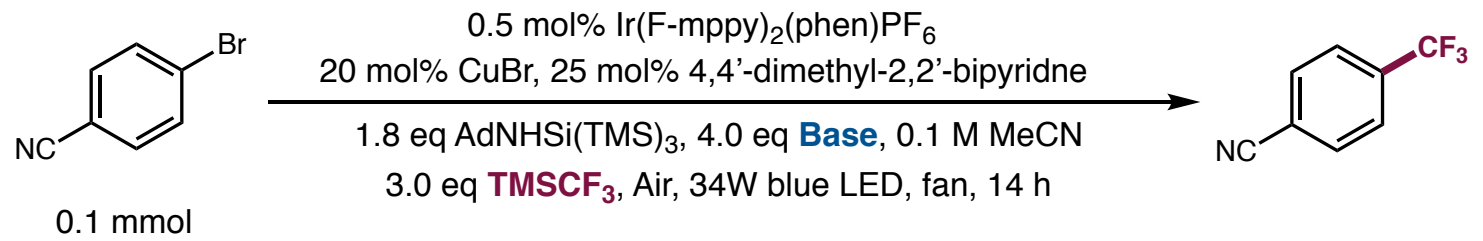

\begin{tabular}{cc} 
Base & Yield \\
\hline $\mathrm{NaOAc}$ & $87 \%$ \\
$\mathrm{LiOAc}$ & $65 \%$ \\
$\mathrm{KOAC}$ & $33 \%$ \\
$\mathrm{Na}_{2} \mathrm{CO}_{3}$ & $72 \%$ \\
$\mathrm{~K}_{3} \mathrm{PO}_{4}$ & $54 \%$ \\
$\mathrm{CsF}$ & $30 \%$ \\
$\mathrm{t}-\mathrm{BuOK}$ & $0 \%$ \\
$\mathrm{DBU}$ & $35 \%$ \\
$\mathrm{BTMG}$ & $38 \%$ \\
TMG & $50 \%$ \\
Pyridine & $68 \%$ \\
$2,6-$ lutidine & $65 \%$
\end{tabular}

Figure S5. Evaluation of different bases. Yields determined by ${ }^{19} \mathrm{~F}$ NMR. 


\section{(b) Optimization of pentafluoroethylation of aryl bromides}

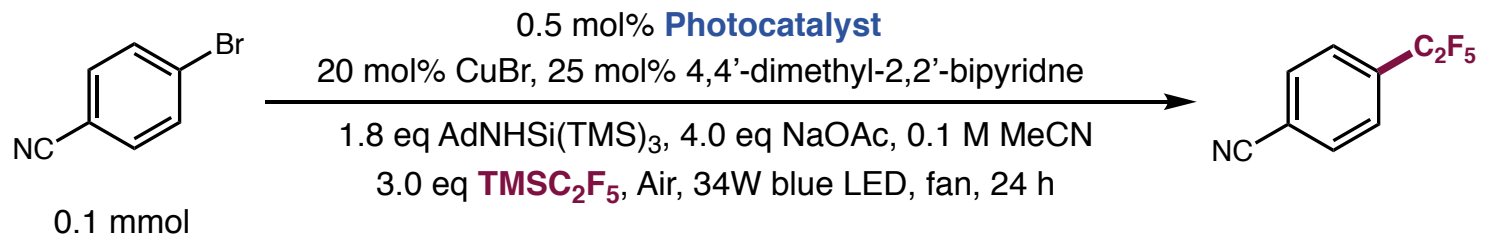

\begin{tabular}{cc} 
Photocatalyst & Yield \\
\hline $\operatorname{Ir}(\mathrm{ppy})_{2}(\mathrm{bpy}) \mathrm{PF}_{6}$ & $68 \%$ \\
$\operatorname{Ir}(\mathrm{ppy})_{2}(\mathrm{dtbbpy}) \mathrm{PF}_{6}$ & $55 \%$ \\
$\operatorname{Ir}(\mathrm{F}-\mathrm{mppy})_{2}(\mathrm{phen}) \mathrm{PF}_{6}$ & $48 \%$ \\
$\operatorname{Ir}\left(\mathrm{F}-\mathrm{mppy}_{2}(\mathrm{dtbbpy}) \mathrm{PF}_{6}\right.$ & $53 \%$ \\
$\operatorname{Ir}\left(\mathrm{dF}\left(\mathrm{CF}_{3}\right) \mathrm{ppy}\right)_{2}(\mathrm{dtbbpy}) \mathrm{PF}_{6}$ & $35 \%$ \\
$\operatorname{Ir}\left(\mathrm{dF}\left(\mathrm{CF}_{3}\right) \mathrm{ppy}_{2}\left(\mathrm{dCF}_{3} \mathrm{bpy}\right) \mathrm{PF}_{6}\right.$ & $42 \%$
\end{tabular}

Figure S6. Evaluation of different photocatalysts. Yields determined by ${ }^{19} \mathrm{~F}$ NMR.

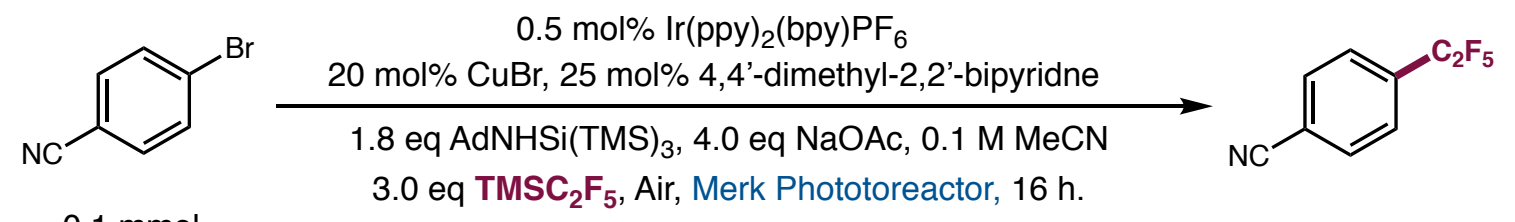

$0.1 \mathrm{mmol}$ Fan speed: $5200 \mathrm{rpm}$, Stir rate: 500 rpm, light intensity: $\mathbf{X X} \%$

\begin{tabular}{ccccc} 
Intensity & Yield & $A r-B r$ & $A r-H$ & $A r-A r$ \\
\hline $25 \%$ & $52 \%$ & $28 \%$ & $15 \%$ & $0 \%$ \\
$35 \%$ & $68 \%$ & $20 \%$ & $12 \%$ & $0 \%$ \\
$45 \%$ & $85 \%$ & $10 \%$ & $3 \%$ & $0 \%$ \\
$55 \%$ & $78 \%$ & $8 \%$ & $12 \%$ & $0 \%$ \\
$65 \%$ & $66 \%$ & $2 \%$ & $20 \%$ & $5 \%$ \\
$80 \%$ & $43 \%$ & $0 \%$ & $30 \%$ & $14 \%$ \\
$100 \%$ & $35 \%$ & $0 \%$ & $35 \%$ & $22 \%$
\end{tabular}

Figure S7. Evaluation of light intensity on the Penn PhD Photoreactor. Yields determined by ${ }^{19} \mathrm{~F}$ NMR analysis. 


\section{(c) Optimization of heptafluoropropylation of aryl bromides}

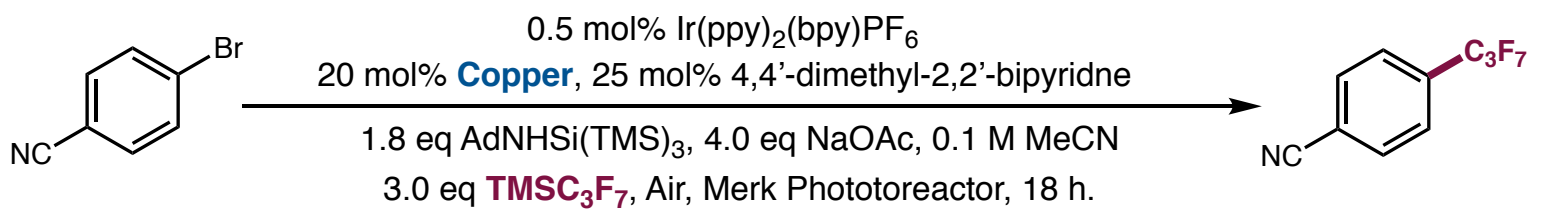

$0.1 \mathrm{mmol}$

Fan speed: $5200 \mathrm{rpm}$, Stir rate: $500 \mathrm{rpm}$, light intensity: 40\%

\begin{tabular}{cc} 
Copper & Yield \\
\hline $\mathrm{CuBr}$ & $33 \%$ \\
$\mathrm{Cul}$ & $35 \%$ \\
$\mathrm{Cu}(\mathrm{MeCN})_{4} \mathrm{BF}_{4}$ & $44 \%$ \\
$\mathrm{CuTc}$ & $27 \%$
\end{tabular}

Figure S8. Evaluation of copper source. Yields determined by ${ }^{19} \mathrm{~F}$ NMR analysis.

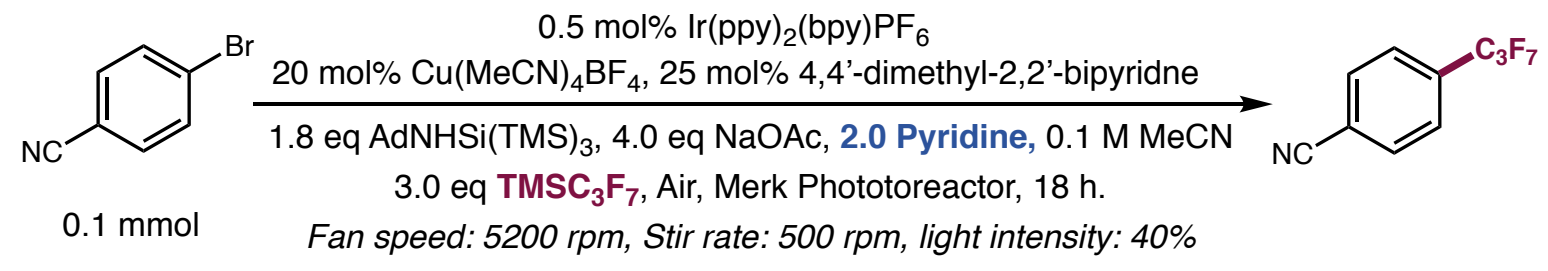

\begin{tabular}{cc} 
Deviation & Yield \\
\hline None & $55 \%$ \\
No pyridine & $44 \%$ \\
4.0 pyridine instead of NaOAc & $13 \%$ \\
2.0 pyridine instead of ligand & $25 \%$
\end{tabular}

Figure S9. Evaluation of pyridine as an additive. Yields determined by ${ }^{19} \mathrm{~F}$ NMR analysis. 


\section{4) General Procedures and Experimental Data}

(a) General procedure for trifluoromethylation: To an oven-dried 40-mL vial equipped with a cross shaped stir bar were added AdNHSi(TMS $)_{3}(0.9 \mathrm{mmol}, 358 \mathrm{mg}, 1.8$ equiv.), $\mathrm{NaOAc}(2.0$ mmol, $164 \mathrm{mg}, 4.0$ equiv.), aryl bromide ( $0.5 \mathrm{mmol}, 1.0$ equiv.), $\mathrm{CuBr}(0.1 \mathrm{mmol}, 14.3 \mathrm{mg}, 0.2$ eq), ligand (0.125 mmol, 0.25 equiv.) and photocatalyst $\operatorname{Ir}\left[(\mathrm{F}-\mathrm{mppy})_{2}(\mathrm{phen}) \mathrm{PF}_{6}(2.2 \mathrm{mg}, 2.5\right.$ $\mu \mathrm{mol}, 0.005$ equiv.). Then, $\mathrm{MeCN}(2.5 \mathrm{~mL}, 0.2 \mathrm{M}$ ) was added via syringe, followed by addition of $\mathrm{TMSCF}_{3}(220 \mu \mathrm{L}, 1.5 \mathrm{mmol}, 3.0$ equiv.). After the reaction mixture was stirred for $5 \mathrm{~min}$, the vial septum was pierced by an $18 \mathrm{G}$ needle, which was left in the septum for the duration of the reaction to allow for air flow without solvent evaporation. The reaction vial was then irradiated with two 36W Kessil H150-Blue (maximum blue with maximum intensity setting) from $6 \mathrm{~cm}$ away with fan cooling for $16 \mathrm{~h}$, or Integrated Photoreactor for $14 \mathrm{~h}$ under the following conditions: $40 \%$ LED intensity, $500 \mathrm{rpm}$ stirring rate and $5200 \mathrm{rpm}$ fan speed. Work up and purification for each substrate are described below.

When scaling up the reaction, we found that the concentration was very important for this reaction to preserve high reaction efficiency (Figure S10).

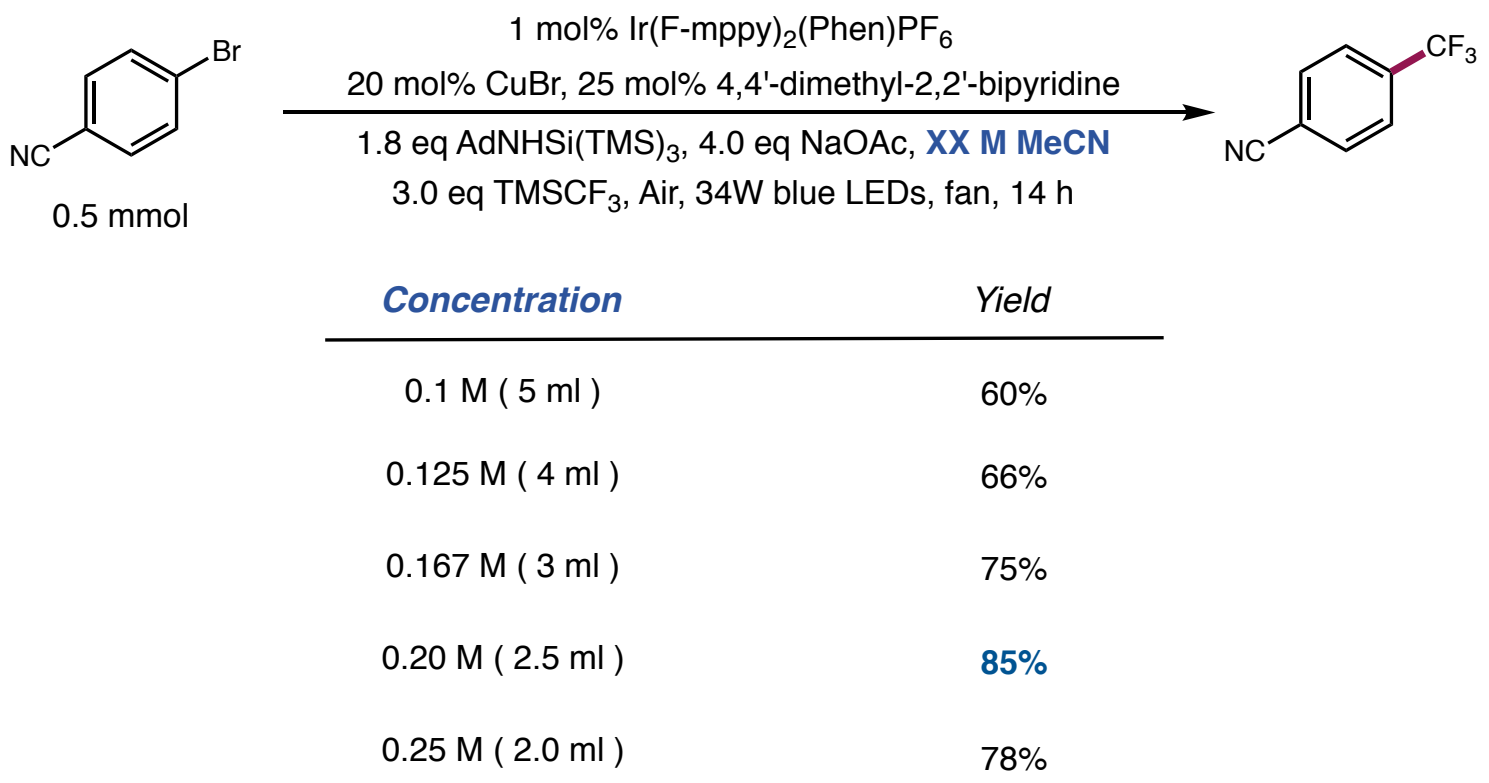

Figure S10. Evaluation of concentration. Yields determined by ${ }^{19} \mathrm{~F}$ NMR analysis. 


\section{4-(trifluoromethyl)benzonitrile (14)}

$\mathrm{NCF}_{3}^{\mathrm{CF}_{3}}$

Prepared following the general procedure outlined above using $\operatorname{Ir}\left[(\mathrm{F}-\mathrm{mppy})_{2}(\mathrm{phen})\right] \mathrm{PF}_{6}(2.2 \mathrm{mg}$, $2.5 \mu \mathrm{mol}, 0.005$ equiv.), $\mathrm{CuBr}$ (14.3 mg, $0.1 \mathrm{mmol}, 0.20$ equiv.), 4,4'-dimethyl-2,2'-bipyridine (23 $\mathrm{mg}, 0.125 \mathrm{mmol}, 0.25$ equiv.), AdNHSi(TMS) 3 ( $358 \mathrm{mg}, 0.9 \mathrm{mmol}, 1.8$ equiv.), $\mathrm{NaOAc}(164 \mathrm{mg}$, $2.0 \mathrm{mmol}, 4.0$ equiv.), 4-bromobenzonitrile (91.0 mg, $0.5 \mathrm{mmol}, 1.0$ equiv.), $\mathrm{TMSCF}_{3}$ reagent $(220 \mu \mathrm{L}, 1.5 \mathrm{mmol}, 3.0$ equiv.) and $\mathrm{MeCN}(2.5 \mathrm{~mL}, 0.2 \mathrm{M})$.

The final reaction mixture was diluted with EtOAc $(20 \mathrm{~mL})$ and then filtered over celite. After washing the celite with EtOAc three times, the organic solution was concentrated to yield the crude product as an oil. The product was purified via silica gel column chromatography (gradient 5 to $20 \%$ DCM in pentane) to yield the white solid ( $72 \mathrm{mg}, 85 \%$ yield).

${ }^{1} \mathbf{H}$ NMR (500 MHz, CDCl $) \delta 7.81(\mathrm{~d}, \mathrm{~J}=8.3 \mathrm{~Hz}, 2 \mathrm{H}), 7.75(\mathrm{~d}, J=8.2 \mathrm{~Hz}, 2 \mathrm{H})$.

${ }^{13}$ C NMR (125 MHz, CDCl $)$ ) $\delta 134.61(\mathrm{q}, J=33.3 \mathrm{~Hz}), 132.79,126.27(\mathrm{q}, J=3.8 \mathrm{~Hz}), 123.15(\mathrm{q}$, $J=273.0 \mathrm{~Hz}), 117.55,116.15$.

${ }^{19}$ F NMR (282 MHz, $\left.\mathbf{C D C l}_{3}\right) \delta-63.59(\mathrm{~s}, 3 \mathrm{~F})$.

Data are consistent with those reported in the literature: Shi G.; Shao, C.; Pan, S.; Yu, J.; Zhang, Y. Org. Lett. 2015, 17, 38. 


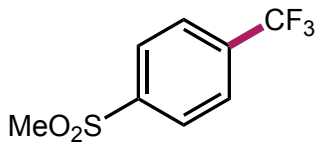

1-(methylsulfonyl)-4-(trifluoromethyl)benzene (16)

Prepared following the general procedure outlined above using $\operatorname{Ir}\left[(\mathrm{F}-\mathrm{mppy})_{2}(\mathrm{phen}) \mathrm{PF}_{6}(2.2 \mathrm{mg}\right.$, $2.5 \mu \mathrm{mol}, 0.005$ equiv.), $\mathrm{CuBr}$ (14.3 mg, $0.1 \mathrm{mmol}, 0.20$ equiv.), 4,4'-dimethyl-2,2'-bipyridine (23 mg, 0.125 mmol, 0.25 equiv.), AdNHSi(TMS) 3 (358 mg, 0.9 mmol, 1.8 equiv.), NaOAc (164 mg, $2.0 \mathrm{mmol}, 4.0$ equiv.), 1-bromo-4-(methylsulfonyl)benzene (120 mg, $0.5 \mathrm{mmol}, 1.0$ equiv.), $\mathrm{TMSCF}_{3}$ reagent $(220 \mu \mathrm{L}, 1.5 \mathrm{mmol}, 3.0$ equiv.) and $\mathrm{MeCN}(2.5 \mathrm{~mL}, 0.2 \mathrm{M})$.

The final reaction mixture was diluted with EtOAc $(20 \mathrm{~mL})$ and then filtered over celite. After washing the celite with EtOAc three times, the organic solution was concentrated to yield the crude product as an oil. The product was purified via silica gel column chromatography (gradient 5 to $40 \%$ DCM in pentane) to yield the white solid ( $89 \mathrm{mg}, 80 \%$ yield).

${ }^{1}$ H NMR (500 MHz, CDCl $) \delta 8.09(\mathrm{~d}, J=8.0 \mathrm{~Hz}, 2 \mathrm{H}), 7.85(\mathrm{~d}, J=8.1 \mathrm{~Hz}, 2 \mathrm{H}), 3.09$ (s, 3H).

${ }^{13}$ C NMR (125 MHz, $\left.\mathbf{C D C l}_{3}\right) \delta 144.08,135.61$ (q, $\left.J=33.1 \mathrm{~Hz}\right), 128.22,126.70(\mathrm{q}, J=3.8 \mathrm{~Hz})$, $123.20(\mathrm{q}, J=273.1 \mathrm{~Hz}), 44.45$.

${ }^{19}$ F NMR (282 MHz, CDCl 3$) \delta-63.25$ (s, 3F).

Data are consistent with those reported in the literature: Shavnya, A.; Coffey, S. B.; Smith, A. C.; Mascitti, V. Org. Lett. 2013, 15, 6226. 


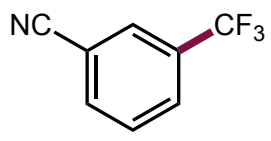

\section{3-(trifluoromethyl)benzonitrile (17)}

Prepared following the general procedure outlined above using $\operatorname{Ir}\left[(\mathrm{F}-\mathrm{mppy})_{2}(\mathrm{phen}) \mathrm{PF}_{6}(2.2 \mathrm{mg}\right.$, $2.5 \mu \mathrm{mol}, 0.005$ equiv.), $\mathrm{CuBr}$ (14.3 mg, $0.1 \mathrm{mmol}, 0.20$ equiv.), 4,4'-dimethyl-2,2'-bipyridine (23 mg, 0.125 mmol, 0.25 equiv.), AdNHSi(TMS) 3 (358 mg, 0.9 mmol, 1.8 equiv.), NaOAc (164 mg, $2.0 \mathrm{mmol}, 4.0$ equiv.), 3-bromobenzonitrile (91 mg, $0.5 \mathrm{mmol}, 1.0$ equiv.), $\mathrm{TMSCF}_{3}$ reagent (220 $\mu \mathrm{L}, 1.5 \mathrm{mmol}, 3.0$ equiv.) and $\mathrm{MeCN}(2.5 \mathrm{~mL}, 0.2 \mathrm{M})$.

The final reaction mixture was diluted with $\mathrm{Et}_{2} \mathrm{O}(20 \mathrm{~mL})$ and then filtered over celite. After washing the celite with $\mathrm{Et}_{2} \mathrm{O}$ three times, the organic solution was concentrated to yield the crude product as an oil. The product was purified via silica gel column chromatography (gradient 1 to $10 \% \mathrm{Et}_{2} \mathrm{O}$ in pentane) to yield the pure oil (71 $\mathrm{mg}, 84 \%$ yield).

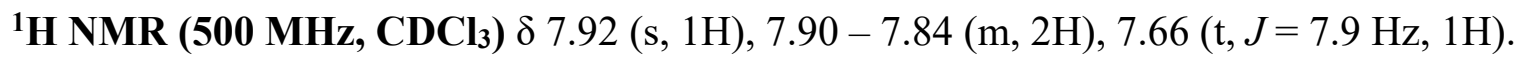

${ }^{13}$ C NMR (125 MHz, CDCl $) \delta 135.42(\mathrm{~d}, J=1.4 \mathrm{~Hz}), 132.12$ (q, $\left.J=33.8 \mathrm{~Hz}\right), 130.10,129.62$ $(\mathrm{q}, J=3.6 \mathrm{~Hz}), 129.14(\mathrm{q}, J=3.9 \mathrm{~Hz}), 123.00$ (q, $J=272.7 \mathrm{~Hz}), 117.45,113.59$.

${ }^{19}$ F NMR (376 MHz, CDCl 3$) \delta-63.25(\mathrm{~s}, 3 \mathrm{~F})$.

Data are consistent with those reported in the literature: Shi, S.; Szostak, M. Org. Lett. 2017, 19, 3095 . 


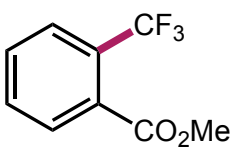

methyl 2-(trifluoromethyl)benzoate (18)

Prepared following the general procedure outlined above using $\operatorname{Ir}\left[(\mathrm{F}-\mathrm{mppy})_{2}(\mathrm{phen})\right] \mathrm{PF}_{6}(2.2 \mathrm{mg}$, $2.5 \mu \mathrm{mol}, 0.005$ equiv.), $\mathrm{CuBr}$ ( $14.3 \mathrm{mg}, 0.1 \mathrm{mmol}, 0.20$ equiv.), 4,4'-dimethyl-2,2'-bipyridine (23 mg, 0.125 mmol, 0.25 equiv.), AdNHSi(TMS) 3 (358 mg, 0.9 mmol, 1.8 equiv.), NaOAc (164 mg, $2.0 \mathrm{mmol}$, 4.0 equiv.), methyl 2-bromobenzoate (108 mg, $0.5 \mathrm{mmol}, 1.0$ equiv.), $\mathrm{TMSCF}_{3}$ reagent $(220 \mu \mathrm{L}, 1.5 \mathrm{mmol}, 3.0$ equiv.) and $\mathrm{MeCN}(2.5 \mathrm{~mL}, 0.2 \mathrm{M})$.

The final reaction mixture was diluted with $\mathrm{Et}_{2} \mathrm{O}(20 \mathrm{~mL})$ and then filtered over celite. After washing the celite with $\mathrm{Et}_{2} \mathrm{O}$ three times, the organic solution was concentrated to yield the crude product as an oil. The product was purified via silica gel column chromatography (gradient 5 to $30 \%$ DCM in pentane) to yield the pure product as an oil (71 $\mathrm{mg}, 70 \%$ yield).

${ }^{1}$ H NMR (500 MHz, CDCl $) \delta 7.82-7.69(\mathrm{~m}, 2 \mathrm{H}), 7.66-7.56(\mathrm{~m}, 2 \mathrm{H}), 3.94(\mathrm{~s}, 3 \mathrm{H})$.

${ }^{13}$ C NMR (125 MHz, CDCl $) \delta$ 167.42, 131.85, 131.31, 131.20 (q, $\left.J=2.1 \mathrm{~Hz}\right), 130.29,128.92$ (q, $J=32.4 \mathrm{~Hz}), 126.82$ (q, $J=5.3 \mathrm{~Hz}), 123.47$ (q, $J=273.3 \mathrm{~Hz}), 52.97$.

${ }^{19}$ F NMR (376 MHz, $\left.\mathbf{C D C l}_{3}\right) \delta-59.75(\mathrm{~s}, 3 \mathrm{~F})$.

Data are consistent with those reported in the literature: Lishchynskyi, A.; Novikov, M. A.; Martin, E.; Grushin, V. V. J. Org. Chem. 2013, 78, 11126. 


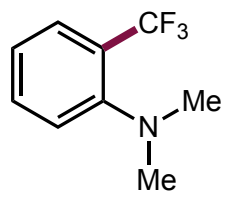

\section{$N, N$-dimethyl-2-(trifluoromethyl)aniline (19)}

Prepared following the general procedure outlined above using $\operatorname{Ir}\left[(\mathrm{F}-\mathrm{mppy})_{2}\right.$ (phen) $] \mathrm{PF}_{6}(2.2 \mathrm{mg}$, $2.5 \mu \mathrm{mol}, 0.005$ equiv.), $\mathrm{CuBr}$ (14.3 mg, $0.1 \mathrm{mmol}, 0.20$ equiv.), 4,4'-dimethyl-2,2'-bipyridine (23 mg, 0.125 mmol, 0.25 equiv.), AdNHSi(TMS) 3 (358 mg, 0.9 mmol, 1.8 equiv.), NaOAc (164 mg, $2.0 \mathrm{mmol}, 4.0$ equiv.), 2-bromo- $N, N$-dimethylaniline (100 mg, $75 \mu \mathrm{l}, 0.5 \mathrm{mmol}, 1.0$ equiv.), $\mathrm{TMSCF}_{3}$ reagent $(220 \mu \mathrm{L}, 1.5 \mathrm{mmol}, 3.0$ equiv.) and $\mathrm{MeCN}(2.5 \mathrm{ml}, 0.2 \mathrm{M})$.

***Yield was determined by ${ }^{19} \mathrm{~F}$ NMR in duplicate due to the high volatility of the desired product.***

1,4-Difluorobenzene ( $52 \mu \mathrm{L}, 0.5 \mathrm{mmol}, 1.0$ equiv.) was added as the internal standard for ${ }^{19} \mathrm{~F}$ NMR analysis (DMSO- $d_{6}$ ). (65\% yield - average of two trials: $67 \%$ yield, $63 \%$ yield).

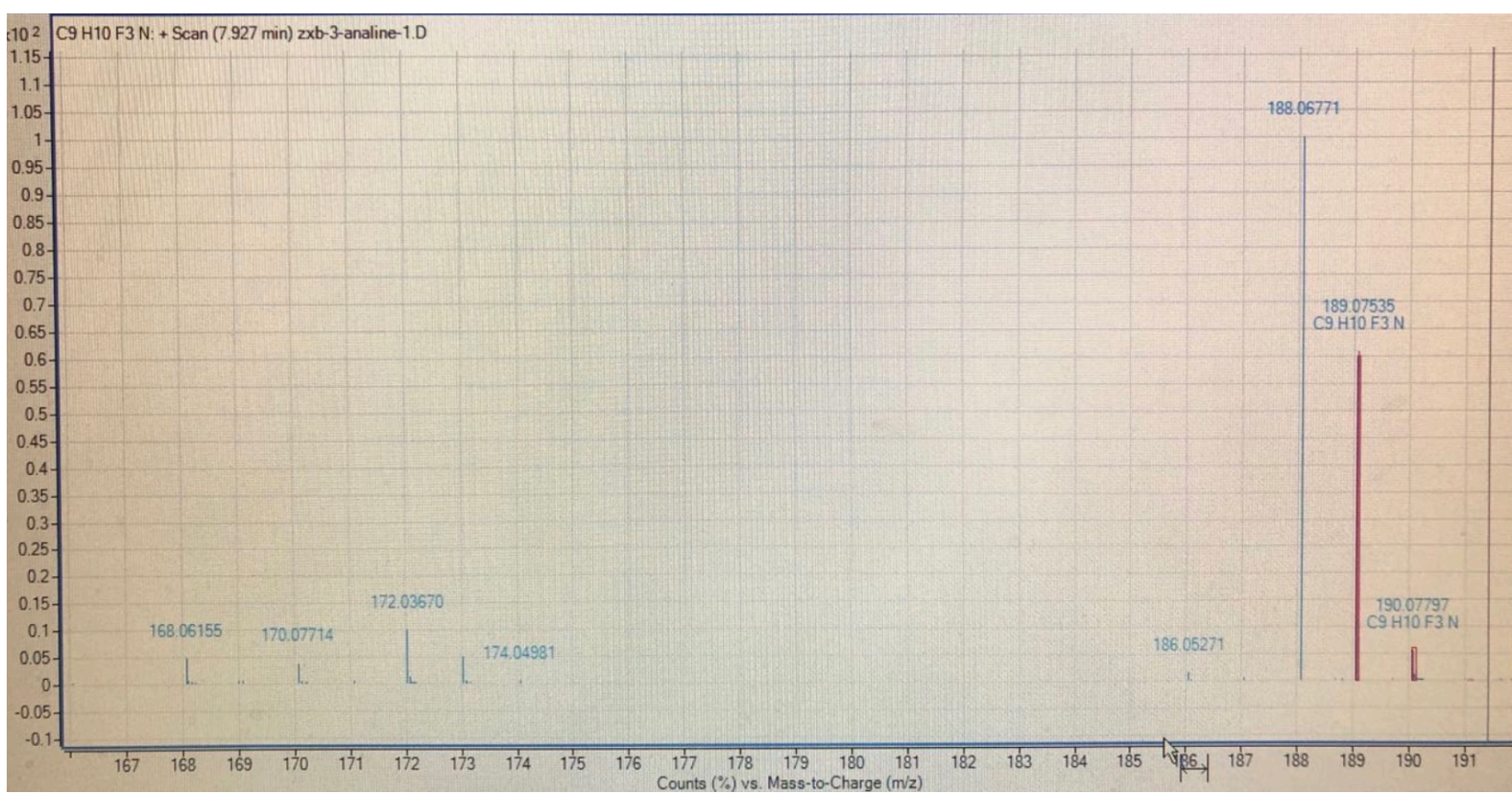

HRMS (GC-EI-TOF) m/z calcd. for $\mathrm{C}_{9} \mathrm{H}_{10} \mathrm{~F}_{3} \mathrm{~N}\left(\left[\mathrm{M}^{*}\right]^{+}\right)$189.0759, found 189.0754 . 


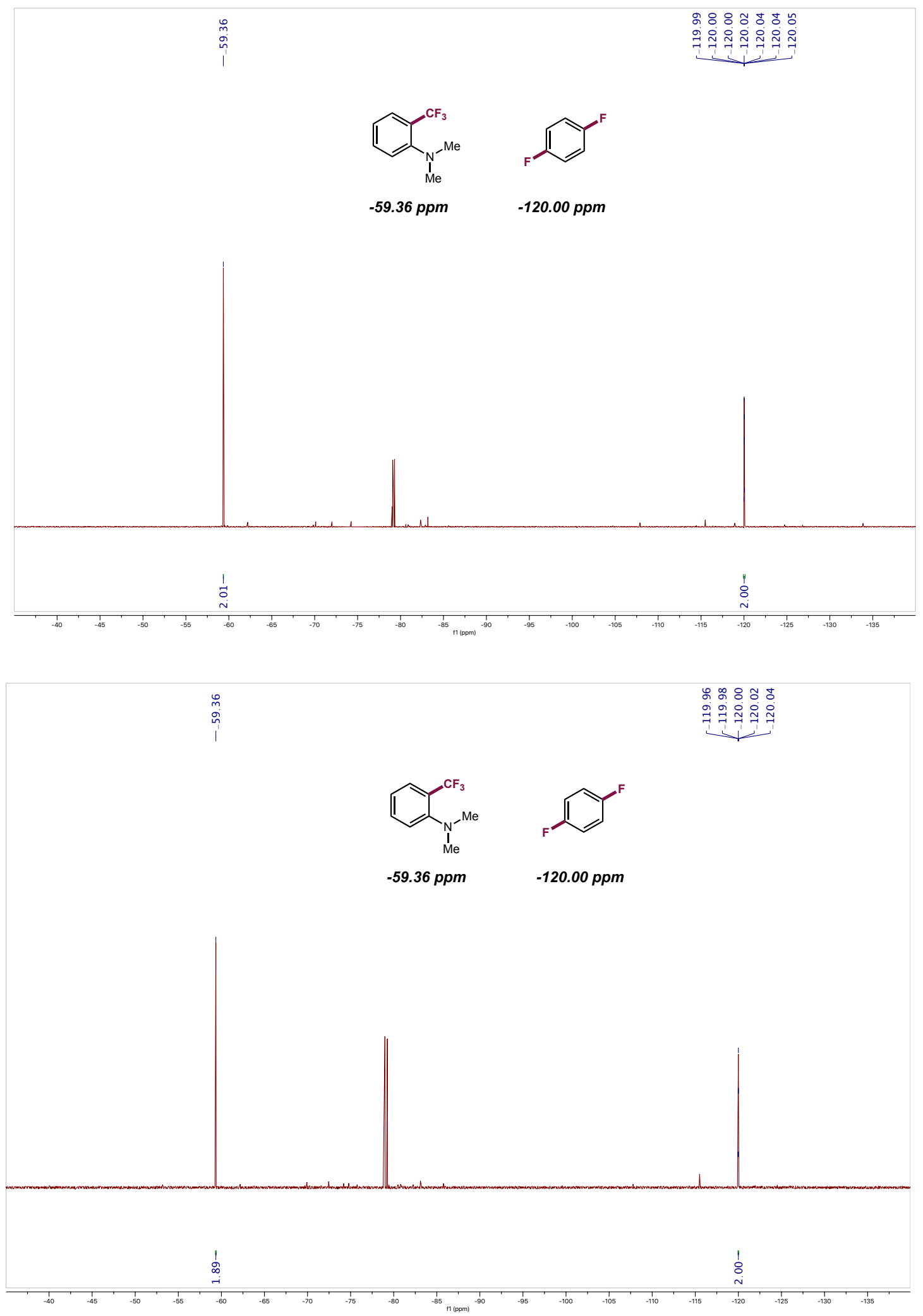

Figure S11. ${ }^{19} \mathrm{~F}$ NMR assay for $N, N$-dimethyl-2-(trifluoromethyl)aniline (19) 


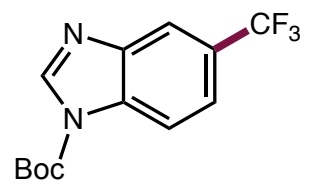

tert-butyl 5-(trifluoromethyl)-1H-benzo[d]imidazole-1-carboxylate (20)

Prepared following the general procedure outlined above using $\operatorname{Ir}\left[(\mathrm{F}-\mathrm{mppy})_{2}(\mathrm{phen})\right] \mathrm{PF}_{6}(2.2 \mathrm{mg}$, $2.5 \mu \mathrm{mol}, 0.005$ equiv.), $\mathrm{CuBr}$ (14.3 mg, $0.1 \mathrm{mmol}, 0.20$ equiv.), 4,4'-dimethyl-2,2'-bipyridine (23 mg, 0.125 mmol, 0.25 equiv.), AdNHSi(TMS) $)_{3}(358 \mathrm{mg}, 0.9$ mmol, 1.8 equiv.), NaOAc (164 mg, $2.0 \mathrm{mmol}, 4.0$ equiv.), tert-butyl 5-bromo- $1 H$-benzo[ $d]$ imidazole-1-carboxylate (150 mg, 0.5 mmol, 1.0 equiv.), $\mathrm{TMSCF}_{3}$ reagent $(220 \mu \mathrm{L}, 1.5 \mathrm{mmol}, 3.0$ equiv.) and $\mathrm{MeCN}(2.5 \mathrm{~mL}, 0.2 \mathrm{M})$.

The final reaction mixture was diluted with EtOAc $(20 \mathrm{~mL})$ and then filtered over celite. After washing the celite with EtOAc three times, the organic solution was concentrated to yield the crude product as an oil. The product was purified via silica gel column chromatography (gradient 5 to $40 \%$ DCM in pentane) to yield the product a white solid (97 $\mathrm{mg}, 68 \%$ yield).

H NMR (500 MHz, DMSO-d $) \delta 8.84(\mathrm{~s}, 1 \mathrm{H}), 8.15-8.11(\mathrm{~m}, 2 \mathrm{H}), 7.78(\mathrm{dd}, J=8.6,1.3 \mathrm{~Hz}$, $1 \mathrm{H}), 1.66(\mathrm{~s}, 9 \mathrm{H})$.

${ }^{13}$ C NMR (125 MHz, DMSO-d6) $\delta$ 147.22, 145.09, 143.46, 133.58, 124.50 (q, $\left.J=271.8 \mathrm{~Hz}\right)$, 124.95 (q, $J=31.9 \mathrm{~Hz}), 121.87$ (q, $J=3.6 \mathrm{~Hz}), 117.55$ (q, $J=4.1 \mathrm{~Hz}), 115.23,86.19,27.54$.

${ }^{19}$ F NMR (282 MHz, DMSO-d $\left.{ }_{6}\right) \delta-59.49(\mathrm{~s}, 3 \mathrm{~F})$.

Data are consistent with those reported in the literature: Le, C.; Chen, T. Q.; Liang, T.; Zhang, P.; MacMillan, D. W. C. Science 2018, 360, 1010. 


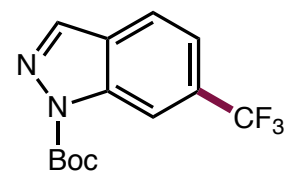

tert-butyl 6-(trifluoromethyl)-1H-indazole-1-carboxylate (21)

Prepared following the general procedure outlined above using $\operatorname{Ir}\left[(\mathrm{F}-\mathrm{mppy})_{2}\right.$ (phen) $] \mathrm{PF}_{6}(2.2 \mathrm{mg}$, $2.5 \mu \mathrm{mol}, 0.005$ equiv.), $\mathrm{CuBr}$ (14.3 mg, $0.1 \mathrm{mmol}, 0.20$ equiv.), 4,4'-dimethyl-2,2'-bipyridine (23 mg, 0.125 mmol, 0.25 equiv.), AdNHSi(TMS) 3 (358 mg, 0.9 mmol, 1.8 equiv.), NaOAc (164 mg, $2.0 \mathrm{mmol}, 4.0$ equiv.), tert-butyl 6-bromo- $1 H$-indazole-1-carboxylate (150 mg, $0.5 \mathrm{mmol}, 1.0$ equiv.), $\mathrm{TMSCF}_{3}$ reagent $(220 \mu \mathrm{L}, 1.5 \mathrm{mmol}, 3.0$ equiv.) and $\mathrm{MeCN}(2.5 \mathrm{~mL}, 0.2 \mathrm{M})$.

The final reaction mixture was diluted with EtOAc $(20 \mathrm{~mL})$ and then filtered over celite. After washing the celite with EtOAc three times, the organic solution was concentrated to yield the crude product as an oil. The product was purified via silica gel column chromatography (gradient 1 to $10 \%$ DCM in pentane) to yield the product as a white solid (100 $\mathrm{mg}, 72 \%$ yield).

${ }^{1}$ H NMR (500 MHz, CDCl$) ~ \delta 8.56(s, 1 H), 8.25(\mathrm{~s}, 1 \mathrm{H}), 7.86(\mathrm{~d}, J=8.3 \mathrm{~Hz}, 1 \mathrm{H}), 7.57(\mathrm{dd}, J=$ 8.4, $1.5 \mathrm{~Hz}, 1 \mathrm{H}), 1.74(\mathrm{~s}, 9 \mathrm{H})$.

${ }^{13}$ C NMR (125 MHz, $\left.\mathbf{C D C l}_{3}\right) \delta$ 148.93, 139.21, 139.12, 131.04 (q, $\left.J=32.3 \mathrm{~Hz}\right), 127.80,124.26$ $(\mathrm{q}, J=272.8 \mathrm{~Hz}), 122.01,120.53(\mathrm{q}, J=3.3 \mathrm{~Hz}), 112.62(\mathrm{q}, J=4.7 \mathrm{~Hz}), 85.96,28.25$.

${ }^{19}$ F NMR (376 MHz, CDCl 3$) \delta-61.84(\mathrm{~s}, 3 \mathrm{~F})$.

Data are consistent with those reported in the literature: Le, C.; Chen, T. Q.; Liang, T.; Zhang, P.; MacMillan, D. W. C. Science 2018, 360, 1010. 


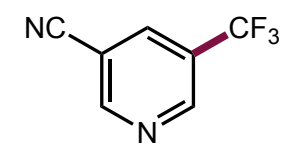

\section{5-(trifluoromethyl)nicotinonitrile (22)}

Prepared following the general procedure outlined above using $\operatorname{Ir}\left[(\mathrm{F}-\mathrm{mppy})_{2}(\mathrm{phen})\right] \mathrm{PF}_{6}(2.2 \mathrm{mg}$, $2.5 \mu \mathrm{mol}, 0.005$ equiv.), $\mathrm{CuBr}$ (14.3 mg, $0.1 \mathrm{mmol}, 0.20$ equiv.), 4,4'-dimethyl-2,2'-bipyridine (23 mg, 0.125 mmol, 0.25 equiv.), AdNHSi(TMS) 3 ( $358 \mathrm{mg}, 0.9$ mmol, 1.8 equiv.), NaOAc (164 mg, $2.0 \mathrm{mmol}, 4.0$ equiv.), 5-bromonicotinonitrile ( $92 \mathrm{mg}, 0.5 \mathrm{mmol}, 1.0$ equiv.), $\mathrm{TMSCF}_{3}$ reagent $(220 \mu \mathrm{L}, 1.5 \mathrm{mmol}, 3.0$ equiv.) and $\mathrm{MeCN}(2.5 \mathrm{~mL}, 0.2 \mathrm{M})$.

The final reaction mixture was diluted with $\mathrm{Et}_{2} \mathrm{O}(20 \mathrm{~mL})$ and then filtered over celite. After washing the celite with $\mathrm{Et}_{2} \mathrm{O}$ three times, the organic solution was concentrated to yield the crude product as an oil. The product was purified via silica gel column chromatography (gradient 5 to $40 \%$ DCM in pentane) to yield the pure product ( $60 \mathrm{mg}, 70 \%$ yield).

${ }^{1}$ H NMR (400 MHz, CDCl $) \delta 9.11-9.08(\mathrm{~m}, 2 \mathrm{H}), 8.23(\mathrm{~s}, 1 \mathrm{H})$.

${ }^{13} \mathbf{C}$ NMR (125 MHz, $\left.\mathbf{C D C l}_{3}\right) \delta 155.37,149.92(\mathrm{q}, J=3.8 \mathrm{~Hz}), 136.63(\mathrm{t}, J=3.7 \mathrm{~Hz}), 127.37$ (q, $J=34.5 \mathrm{~Hz}), 122.40$ (q, $J=273.0 \mathrm{~Hz}), 115.21,110.60$.

${ }^{19}$ F NMR (376 MHz, $\left.\mathbf{C D C l}_{3}\right) \delta-62.79(\mathrm{~s}, 3 \mathrm{~F})$.

Data are consistent with those reported in the literature: Lin, X.; Hou, C.; Li, H.; Weng, Z. Chem. Eur. J. 2016, 22, 2075. 


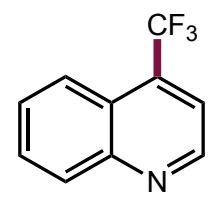

\section{4-(trifluoromethyl)quinoline (23)}

Prepared following the general procedure outlined above using $\operatorname{Ir}\left[(\mathrm{F}-\mathrm{mppy})_{2}(\mathrm{phen})\right] \mathrm{PF}_{6}(2.2 \mathrm{mg}$, $2.5 \mu \mathrm{mol}, 0.005$ equiv.), $\mathrm{CuBr}$ (14.3 mg, $0.1 \mathrm{mmol}, 0.20$ equiv.), 4,4'-dimethyl-2,2'-bipyridine (23 mg, $0.125 \mathrm{mmol}, 0.25$ equiv.), AdNHSi(TMS) 3 ( $358 \mathrm{mg}, 0.9 \mathrm{mmol}, 1.8$ equiv.), NaOAc (164 mg, $2.0 \mathrm{mmol}, 4.0$ equiv.), 4-bromoquinoline (104 mg, $0.5 \mathrm{mmol}, 1.0$ equiv.), $\mathrm{TMSCF}_{3}$ reagent (220 $\mu \mathrm{L}, 1.5 \mathrm{mmol}, 3.0$ equiv.) and $\mathrm{MeCN}(2.5 \mathrm{~mL}, 0.2 \mathrm{M})$.

The final reaction mixture was diluted with $\mathrm{Et}_{2} \mathrm{O}(20 \mathrm{~mL})$ and then filtered over celite. After washing the celite with $\mathrm{Et}_{2} \mathrm{O}$ three times, the organic solution was concentrated to yield the crude

product as an oil. The product was purified via silica gel column chromatography (gradient 5 to $30 \%$ DCM in pentane) to yield the pure product ( $77 \mathrm{mg}, 78 \%$ yield).

${ }^{1} \mathbf{H}$ NMR (400 MHz, CDCl $) \delta 9.03(\mathrm{~d}, J=4.4 \mathrm{~Hz}, 1 \mathrm{H}), 8.22(\mathrm{dd}, J=8.6,1.3 \mathrm{~Hz}, 1 \mathrm{H}), 8.14$ (dt, $J=8.6,2.0 \mathrm{~Hz}, 1 \mathrm{H}), 7.81(\mathrm{t}, J=7.7, \mathrm{~Hz}, 1 \mathrm{H}), 7.73-7.63(\mathrm{~m}, 2 \mathrm{H})$.

${ }^{13}$ C NMR (100 MHz, CDCl 3$) \delta$ 149.67, 149.09, 134.39 (q, $\left.J=31.8 \mathrm{~Hz}\right), 130.56,130.34,128.45$, 124.15 (d, $J=2.3 \mathrm{~Hz}), 123.56$ (q, $J=274.6 \mathrm{~Hz}), 123.08,118.06$ (q, $J=5.3 \mathrm{~Hz})$.

${ }^{19}$ F NMR (376 MHz, $\left.\mathbf{C D C l}_{3}\right) \delta-61.46(\mathrm{~s}, 3 \mathrm{~F})$.

Data are consistent with those reported in the literature: Nagase, M.; Kuninobu, Y.; Kanai, M. J. Am. Chem. Soc. 2016, 138, 6103. 


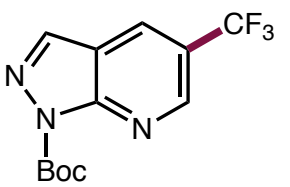

tert-butyl 5-(trifluoromethyl)-1 $H$-pyrazolo[3,4-b]pyridine-1-carboxylate (24)

Prepared following the general procedure outlined above using $\operatorname{Ir}\left[(\mathrm{F}-\mathrm{mppy})_{2}(\mathrm{phen})\right] \mathrm{PF}_{6}(2.2 \mathrm{mg}$, $2.5 \mu \mathrm{mol}, 0.005$ equiv.), $\mathrm{CuBr}$ (14.3 mg, $0.1 \mathrm{mmol}, 0.20$ equiv.), 4,4'-dimethyl-2,2'-bipyridine (23 mg, 0.125 mmol, 0.25 equiv.), AdNHSi(TMS) 3 (358 mg, 0.9 mmol, 1.8 equiv.), NaOAc (164 mg, $2.0 \mathrm{mmol}, 4.0$ equiv.), tert-butyl 5-bromo-1H-pyrazolo[3,4-b]pyridine-1-carboxylate (150 mg, 0.5 mmol, 1.0 equiv.), $\mathrm{TMSCF}_{3}$ reagent $(220 \mu \mathrm{L}, 1.5 \mathrm{mmol}, 3.0$ equiv.) and $\mathrm{MeCN}(2.5 \mathrm{~mL}, 0.2 \mathrm{M})$.

The final reaction mixture was diluted with EtOAc $(20 \mathrm{~mL})$ and then filtered over celite. After washing the celite with EtOAc three times, the organic solution was concentrated to yield the crude product as an oil. The product was purified via silica gel column chromatography (gradient 0 to $3 \%$ acetone in DCM) to yield the pure product as a white crystalline solid (92 $\mathrm{mg}, 65 \%$ yield).

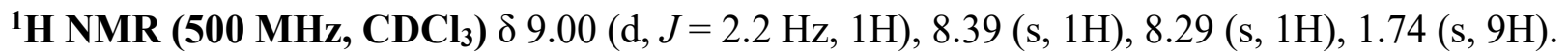

${ }^{13}$ C NMR (125 MHz, CDCl 3$) \delta 152.94,147.83$ (q, $\left.J=3.6 \mathrm{~Hz}\right), 147.47,137.59,128.38$ (q, $J=$ $3.9 \mathrm{~Hz}), 123.77$ (q, $J=272.2 \mathrm{~Hz}), 122.85$ (q, $J=33.4 \mathrm{~Hz}), 116.94,86.46,28.18$.

${ }^{19}$ F NMR (376 MHz, CDCl 3$) \delta-60.88(\mathrm{~s}, 3 \mathrm{~F})$.

Data are consistent with those reported in the literature: Le, C.; Chen, T. Q.; Liang, T.; Zhang, P.; MacMillan, D. W. C. Science 2018, 360, 1010. 


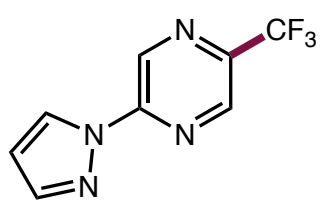

\section{2-(1H-pyrazol-1-yl)-5-(trifluoromethyl)pyrazine (25)}

Prepared following the general procedure outlined above using $\operatorname{Ir}\left[(\mathrm{F}-\mathrm{mppy})_{2}\right.$ (phen) $\mathrm{PF}_{6}(2.2 \mathrm{mg}$, $2.5 \mu \mathrm{mol}, 0.005$ equiv.), $\mathrm{CuBr}$ (14.3 mg, $0.1 \mathrm{mmol}, 0.20$ equiv.), 4,4'-dimethoxy-2,2'-bipyridine (27 mg, 0.125 mmol, 0.25 equiv.), AdNHSi(TMS) $)_{3}$ (358 mg, 0.9 mmol, 1.8 equiv.), NaOAc (164 mg, 2.0 mmol, 4.0 equiv.), 2-bromo-5-(1H-pyrazol-1yl)pyrazine (113 mg, 0.5 mmol, 1.0 equiv.), $\mathrm{TMSCF}_{3}$ reagent $(220 \mu \mathrm{L}, 1.5 \mathrm{mmol}, 3.0$ equiv. $)$ and $\mathrm{MeCN}(2.5 \mathrm{~mL}, 0.2 \mathrm{M})$.

The final reaction mixture was diluted with $\mathrm{Et}_{2} \mathrm{O}(20 \mathrm{~mL})$ and then filtered over celite. After washing the celite with $\mathrm{Et}_{2} \mathrm{O}$ three times, the organic solution was concentrated to yield the crude

product as an oil. The product was purified via silica gel column chromatography (gradient 1 to $10 \%$ DCM in pentane) to yield the pure product as a white crystalline solid ( $71 \mathrm{mg}, 66 \%$ yield).

${ }^{1}$ H NMR (500 MHz, $\left.\mathbf{C D C l}_{3}\right) \delta 9.42(\mathrm{~s}, 1 \mathrm{H}), 8.71(\mathrm{~s}, 1 \mathrm{H}), 8.55(\mathrm{~d}, J=2.7 \mathrm{~Hz}, 1 \mathrm{H}), 7.85(\mathrm{~d}, J=$ $1.7 \mathrm{~Hz}, 1 \mathrm{H}), 6.57(\mathrm{dd}, J=2.8,1.6 \mathrm{~Hz}, 1 \mathrm{H})$.

${ }^{13}$ C NMR (125 MHz, $\left.\mathbf{C D C l}_{3}\right) \delta 148.98,144.41,140.58$ (q, $\left.J=36.0 \mathrm{~Hz}\right), 139.53(\mathrm{~d}, J=3.3 \mathrm{~Hz})$, $135.44,128.23,121.34$ (q, $J=273.6 \mathrm{~Hz}), 109.79$.

${ }^{19}$ F NMR (376 MHz, CDCl 3$) \delta-67.13(\mathrm{~s}, 3 \mathrm{~F})$.

Data are consistent with those reported in the literature: Le, C.; Chen, T. Q.; Liang, T.; Zhang, P.; MacMillan, D. W. C. Science 2018, 360, 1010. 


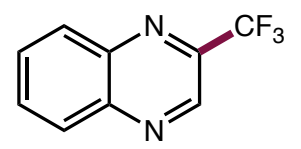

\section{2-(trifluoromethyl)quinoxaline (26)}

Prepared following the general procedure outlined above using $\operatorname{Ir}\left[(\mathrm{F}-\mathrm{mppy})_{2}(\mathrm{phen}) \mathrm{PF}_{6}(2.2 \mathrm{mg}\right.$, $2.5 \mu \mathrm{mol}, 0.005$ equiv.), $\mathrm{CuBr}$ (14.3 mg, $0.1 \mathrm{mmol}, 0.20$ equiv.), 4,4'-dimethoxy-2,2'-bipyridine (27 mg, 0.125 mmol, 0.25 equiv.), AdNHSi(TMS) $)_{3}$ (358 mg, 0.9 mmol, 1.8 equiv.), NaOAc (164 mg, 2.0 mmol, 4.0 equiv.), 2-bromoquinoxaline (105 mg, $0.5 \mathrm{mmol}, 1.0$ equiv.), $\mathrm{TMSCF}_{3}$ reagent (220 $\mu \mathrm{L}, 1.5 \mathrm{mmol}, 3.0$ equiv.) and $\mathrm{MeCN}(2.5 \mathrm{~mL}, 0.2 \mathrm{M})$.

The final reaction mixture was diluted with $\mathrm{Et}_{2} \mathrm{O}(20 \mathrm{~mL})$ and then filtered over celite. After washing the celite with $\mathrm{Et}_{2} \mathrm{O}$ three times, the organic solution was concentrated to yield the crude product as an oil. The product was purified via silica gel column chromatography (gradient 5 to $40 \% \mathrm{DCM}$ in pentane) to yield the pure product as a white crystalline solid ( $81 \mathrm{mg}, 82 \%$ yield).

${ }^{1}$ H NMR (500 MHz, CDCl $) \delta 9.19(\mathrm{~s}, 1 \mathrm{H}), 8.24$ (td, $\left.J=8.1,1.6 \mathrm{~Hz}, 2 \mathrm{H}\right), 7.96-7.90$ (m, 2H).

${ }^{13}$ C NMR (125 MHz, $\left.\mathbf{C D C l}_{3}\right) \delta 143.91,142.95$ (q, $J=35.4$ Hz), 141.07, 141.05, 132.53,131.73, $130.21,129.68,121.27$ (q, $J=275.5 \mathrm{~Hz})$.

${ }^{19}$ F NMR (376 MHz, CDCl 3$) \delta-67.00(\mathrm{~s}, 3 \mathrm{~F})$.

Data are consistent with those reported in the literature: Mormino, M. G.; Fier, P. S.; Hartwig, J. F. Org. Lett. 2014, 16, 1744. 


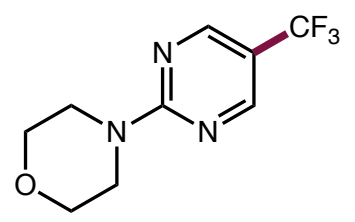

\section{4-(5-(trifluoromethyl)pyrimidin-2-yl)morpholine (27)}

Prepared following the general procedure outlined above using $\operatorname{Ir}\left[(\mathrm{F}-\mathrm{mppy})_{2}(\mathrm{phen})\right] \mathrm{PF}_{6}(2.2 \mathrm{mg}$, $2.5 \mu \mathrm{mol}, 0.005$ equiv.), $\mathrm{CuBr}$ ( $14.3 \mathrm{mg}, 0.1 \mathrm{mmol}, 0.20$ equiv.), 4,4'-dimethyl-2,2'-bipyridine (23 $\mathrm{mg}, 0.125 \mathrm{mmol}, 0.25$ equiv.), AdNHSi(TMS) 3 (358 mg, $0.9 \mathrm{mmol}, 1.8$ equiv.), NaOAc (164 mg, $2.0 \mathrm{mmol}, 4.0$ equiv.), 4-(5-bromopyrimidin-2-yl)morpholine (122 $\mathrm{mg}, 0.5 \mathrm{mmol}, 1.0$ equiv.), $\mathrm{TMSCF}_{3}$ reagent $(220 \mu \mathrm{L}, 1.5 \mathrm{mmol}, 3.0$ equiv.) and $\mathrm{MeCN}(2.5 \mathrm{~mL}, 0.2 \mathrm{M})$.

The final reaction mixture was diluted with EtOAc $(20 \mathrm{~mL})$ and then filtered over celite. After washing the celite with EtOAc three times, the organic solution was concentrated to yield the crude

product as an oil. The product was purified via silica gel column chromatography (gradient 1 to $4 \%$ EA in DCM) to yield the pure product as a white crystalline solid (78 $\mathrm{mg}, 67 \%$ yield).

${ }^{1}$ H NMR (500 MHz, $\left.\mathbf{C D C l}_{3}\right) \delta 8.50(\mathrm{~s}, 2 \mathrm{H}), 3.89(\mathrm{t}, J=4.9 \mathrm{~Hz}, 4 \mathrm{H}), 3.76(\mathrm{t}, J=4.8 \mathrm{~Hz}, 4 \mathrm{H})$.

${ }^{13}$ C NMR (125 MHz, CDCl $) \delta$ 162.30, 155.52 (q, $\left.J=3.5 \mathrm{~Hz}\right), 124.20$ (q, $\left.J=270.0 \mathrm{~Hz}\right), 113.45$ (q, $J=33.9 \mathrm{~Hz}$ ), 66.83, 44.38 .

${ }^{19}$ F NMR (376 MHz, $\left.\mathbf{C D C l}_{3}\right) \delta-61.14$ (s, 3F).

Data are consistent with those reported in the literature: Ge, H. M.; Shen, Q. L. Org. Chem. Front. 2019, 6, 2205. 


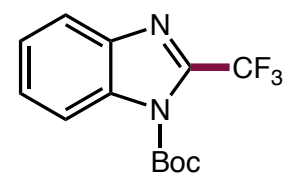

tert-butyl 2-(trifluoromethyl)-1 $H$-benzo[d]imidazole-1-carboxylate (28)

Prepared following the general procedure outlined above using $\operatorname{Ir}\left[(\mathrm{F}-\mathrm{mppy})_{2}(\mathrm{phen}) \mathrm{PF}_{6}(2.2 \mathrm{mg}\right.$, $2.5 \mu \mathrm{mol}, 0.005$ equiv.), $\mathrm{CuBr}$ (14.3 mg, $0.1 \mathrm{mmol}, 0.20$ equiv.), 1,10-phenanthroline (23 mg, 0.125 mmol, 0.25 equiv.), AdNHSi(TMS) 3 (358 mg, 0.9 mmol, 1.8 equiv.), NaOAc (164 mg, 2.0 mmol, 4.0 equiv.), tert-butyl 2-bromo-1H-benzo[d]imidazole-1-carboxylate (149 mg, $0.5 \mathrm{mmol}$, 1.0 equiv.), $\mathrm{TMSCF}_{3}$ reagent $(220 \mu \mathrm{L}, 1.5 \mathrm{mmol}, 3.0$ equiv. $)$ and $\mathrm{MeCN}(2.5 \mathrm{~mL}, 0.2 \mathrm{M})$.

The final reaction mixture was diluted with EtOAc $(20 \mathrm{~mL})$ and then filtered over celite. After washing the celite with EtOAc three times, the organic solution was concentrated to yield the crude product as an oil. The product was purified via silica gel column chromatography (gradient 10 to $50 \%$ DCM in hexane) to yield the pure product ( $92 \mathrm{mg}, 65 \%$ yield).

${ }^{1}$ H NMR (500 MHz, $\left.\mathbf{C D C l}_{3}\right) \delta 8.12(\mathrm{~d}, J=8.3 \mathrm{~Hz}, 1 \mathrm{H}), 7.88(\mathrm{~d}, J=8.1 \mathrm{~Hz}, 1 \mathrm{H}), 7.54-7.49$ $(\mathrm{m}, 1 \mathrm{H}), 7.46-7.41(\mathrm{~m}, 1 \mathrm{H}), 1.70(\mathrm{~s}, 9 \mathrm{H})$.

${ }^{13}$ C NMR (125 MHz, CDCl $) \delta 146.74,140.62$ (q, $\left.J=40.3 \mathrm{~Hz}\right), 140.40,133.88,127.69,125.44$, $121.86,118.61(\mathrm{q}, J=271.1 \mathrm{~Hz}), 115.55,87.62,27.90$.

${ }^{19}$ F NMR (282 MHz, $\left.\mathrm{CDCl}_{3}\right) \delta-61.88(\mathrm{~s}, 3 \mathrm{~F})$.

Data are consistent with those reported in the literature: Le, C.; Chen, T. Q.; Liang, T.; Zhang, P.; MacMillan, D. W. C. Science 2018, 360, 1010. 


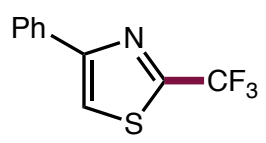

\section{4-phenyl-2-(trifluoromethyl)thiazole (29)}

Prepared following the general procedure outlined above using $\operatorname{Ir}\left[(\mathrm{F}-\mathrm{mppy})_{2}\right.$ (phen) $] \mathrm{PF}_{6}(2.2 \mathrm{mg}$, $2.5 \mu \mathrm{mol}, 0.005$ equiv.), $\mathrm{CuBr}$ ( $14.3 \mathrm{mg}, 0.1 \mathrm{mmol}, 0.20$ equiv.), 1,10-phenanthroline ( $23 \mathrm{mg}$, $0.125 \mathrm{mmol}, 0.25$ equiv.), AdNHSi(TMS) 3 (358 mg, $0.9 \mathrm{mmol}, 1.8$ equiv.), $\mathrm{NaOAc}(164 \mathrm{mg}, 2.0$ mmol, 4.0 equiv.), 2-bromo-4-phenylthiazole (120 mg, $0.5 \mathrm{mmol}, 1.0$ equiv.), TMSCF 3 reagent $(220 \mu \mathrm{L}, 1.5 \mathrm{mmol}, 3.0$ equiv.) and $\mathrm{MeCN}(2.5 \mathrm{~mL}, 0.2 \mathrm{M})$.

The final reaction mixture was diluted with EtOAc $(20 \mathrm{~mL})$ and then filtered over celite. After washing the celite with EtOAc three times, the organic solution was concentrated to yield the crude

product as an oil. The product was purified via silica gel column chromatography (gradient 1 to $10 \% \mathrm{DCM}$ in hexane) to yield the pure product ( $78 \mathrm{mg}, 68 \%$ yield).

${ }^{1}$ H NMR (500 MHz, CDCl $) \delta 7.96-7.88(\mathrm{~m}, 2 \mathrm{H}), 7.69(\mathrm{~s}, 1 \mathrm{H}), 7.46(\mathrm{dd}, J=8.3,6.8 \mathrm{~Hz}, 2 \mathrm{H})$, $7.38-7.42(\mathrm{~m}, 1 \mathrm{H})$.

${ }^{13}$ C NMR (125 MHz, $\left.\mathbf{C D C l}_{3}\right) \delta$ 157.23, 155.72 (q, $\left.J=40.9 \mathrm{~Hz}\right), 133.17,129.19,129.08,126.74$, $119.93(\mathrm{q}, J=272.1 \mathrm{~Hz}), 115.72(\mathrm{q}, J=1.5 \mathrm{~Hz})$.

${ }^{19}$ F NMR (376 MHz, $\left.\mathbf{C D C l}_{3}\right) \delta-61.17$ (s, 3F).

Data are consistent with those reported in the literature: Le, C.; Chen, T. Q.; Liang, T.; Zhang, P.; MacMillan, D. W. C. Science 2018, 360, 1010. 


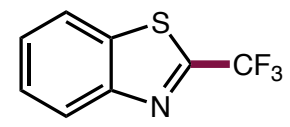

\section{2-(trifluoromethyl)benzo[d] thiazole (30)}

Prepared following the general procedure outlined above using $\operatorname{Ir}\left[(\mathrm{F}-\mathrm{mppy})_{2}\right.$ (phen) $\mathrm{PF}_{6}(2.2 \mathrm{mg}$, $2.5 \mu \mathrm{mol}, 0.005$ equiv.), $\mathrm{CuBr}$ (14.3 mg, $0.1 \mathrm{mmol}, 0.20$ equiv.), 1,10-phenanthroline (23 mg, 0.125 mmol, 0.25 equiv.), AdNHSi(TMS) 3 (358 mg, 0.9 mmol, 1.8 equiv.), NaOAc (164 mg, 2.0 mmol, 4.0 equiv.), 2-bromobenzo[d]thiazole (107 mg, $0.5 \mathrm{mmol}, 1.0$ equiv.), TMSCF 3 reagent (220 $\mu \mathrm{L}, 1.5 \mathrm{mmol}, 3.0$ equiv.) and $\mathrm{MeCN}(2.5 \mathrm{~mL}, 0.2 \mathrm{M})$.

The final reaction mixture was diluted with EtOAc $(20 \mathrm{~mL})$ and then filtered over celite. After washing the celite with EtOAc three times, the organic solution was concentrated to yield the crude product as an oil. The product was purified via silica gel column chromatography (gradient 10 to $40 \%$ DCM in pentane) to yield the pure product ( $74 \mathrm{mg}, 73 \%$ yield).

${ }^{1}$ H NMR (400 MHz, CDCl$) \delta 8.21(\mathrm{dd}, J=8.0,1.4 \mathrm{~Hz}, 1 \mathrm{H}), 8.00(\mathrm{dd}, J=8.0,1.4 \mathrm{~Hz}, 1 \mathrm{H})$, $7.62(\mathrm{t}, J=7.7, \mathrm{~Hz}, 1 \mathrm{H}), 7.57(\mathrm{t}, J=7.6 \mathrm{~Hz}, 1 \mathrm{H})$.

${ }^{13}$ C NMR (125 MHz, CDCl $)$ ) $\delta 156.12(\mathrm{q}, J=40.4$ Hz), 152.24, 135.12, 127.65, 127.52, 125.17, $122.20,119.94(\mathrm{q}, J=273.3 \mathrm{~Hz})$.

${ }^{19}$ F NMR (376 MHz, $\left.\mathrm{CDCl}_{3}\right) \delta-61.72(\mathrm{~s}, 3 \mathrm{~F})$.

Data are consistent with those reported in the literature: Bayarmagnai, B.; Matheis, C.; Risto, E.; Goossen, L. Adv. Synth. Catal. 2014, 356, 2343. 


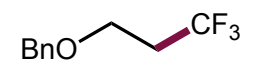

\section{3-Benzyloxy-1,1,1-trifluoropropane (31)}

Prepared following the general procedure outlined above using $\operatorname{Ir}\left[(\mathrm{F}-\mathrm{mppy})_{2}(\mathrm{phen})\right] \mathrm{PF}_{6}(2.2 \mathrm{mg}$, $2.5 \mu \mathrm{mol}, 0.005$ equiv.), $\mathrm{CuBr}$ (14.3 mg, $0.1 \mathrm{mmol}, 0.20$ equiv.), 2,2'-bipyridine (20 mg, 0.125 mmol, 0.25 equiv.), AdNHSi(TMS) 3 (358 mg, 0.9 mmol, 1.8 equiv.), NaOAc (164 mg, 2.0 mmol, 4.0 equiv.), benzyl 2-bromoethylether ( $80 \mu \mathrm{L}, 0.5 \mathrm{mmol}, 1.0$ equiv.), $\mathrm{TMSCF}_{3}$ reagent $(220 \mu \mathrm{L}$, $1.5 \mathrm{mmol}, 3.0$ equiv.) and $\mathrm{MeCN}(2.5 \mathrm{~mL}, 0.2 \mathrm{M})$.

The final reaction mixture was diluted with $\mathrm{Et}_{2} \mathrm{O}(20 \mathrm{~mL})$ and then filtered over celite. After washing the celite with $\mathrm{Et}_{2} \mathrm{O}$ three times, the organic solution was concentrated to yield the crude product as an oil. The product was purified via silica gel column chromatography (gradient 2 to $20 \%$ DCM in pentane) to yield the pure product ( $85 \mathrm{mg}, 84 \%$ yield).

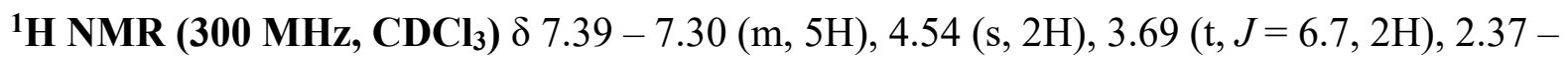
$2.52(\mathrm{~m}, 2 \mathrm{H})$.

${ }^{13}$ C NMR (125 MHz, CDCl $) \delta 137.73,128.63,128.00,127.79,126.27$ (q, $J=276.6$ Hz), 73.40, $63.16(\mathrm{q}, J=3.6 \mathrm{~Hz}), 34.59(\mathrm{q}, J=28.4 \mathrm{~Hz})$.

${ }^{19}$ F NMR (282 MHz, $\left.\mathbf{C D C l}_{3}\right) \delta-64.76(\mathrm{t}, J=10.7 \mathrm{~Hz}, 3 \mathrm{~F})$.

Data are consistent with those reported in the literature: Kornfilt, D. J. P.; MacMillan, D. W. C. J. Am. Chem. Soc. 2019, 141, 6853. 


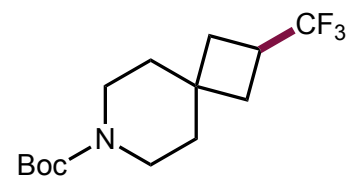

tert-butyl 2-(trifluoromethyl)-7-azaspiro[3.5]nonane-7-carboxylate (32)

Prepared following the general procedure outlined above using $\operatorname{Ir}\left[(\mathrm{F}-\mathrm{mppy})_{2}(\mathrm{phen})\right] \mathrm{PF}_{6}(2.2 \mathrm{mg}$, $2.5 \mu \mathrm{mol}, 0.005$ equiv.), $\mathrm{CuBr}$ (14.3 mg, $0.1 \mathrm{mmol}, 0.20$ equiv.), 2,2'-bipyridine (20 mg, 0.125 mmol, 0.25 equiv.), AdNHSi(TMS) 3 (358 mg, 0.9 mmol, 1.8 equiv.), NaOAc (164 mg, 2.0 mmol, 4.0 equiv.), tert-butyl 2-bromo-7-azaspiro[3.5]nonane-7-carboxylate (152 mg, $0.5 \mathrm{mmol}, 1.0$ equiv.), $\mathrm{TMSCF}_{3}$ reagent $(220 \mu \mathrm{L}, 1.5 \mathrm{mmol}, 3.0$ equiv. $)$ and $\mathrm{MeCN}(2.5 \mathrm{~mL}, 0.2 \mathrm{M})$.

The final reaction mixture was diluted with $\mathrm{Et}_{2} \mathrm{O}(20 \mathrm{~mL})$ and then filtered over celite. After washing the celite with $\mathrm{Et}_{2} \mathrm{O}$ three times, the organic solution was concentrated to yield the crude product as an oil. The product was purified via silica gel column chromatography (gradient 10 to $50 \% \mathrm{DCM}$ in hexane) to yield the pure product (110 $\mathrm{mg}, 75 \%$ yield).

${ }^{1}$ H NMR (300 MHz, CDCl$)$ ) $\delta 3.40$ - 3.29 (m, 2H), 3.32 - 3.22 (m, 2H), 2.89 (dq, J= 18.2, 9.1 $\mathrm{Hz}, 1 \mathrm{H}), 2.03-1.87(\mathrm{~m}, 4 \mathrm{H}), 1.61-1.48(\mathrm{~m}, 4 \mathrm{H}), 1.44$ (s, 9H).

${ }^{13}$ C NMR (125 MHz, CDCl $) \delta 155.00,127.62$ (q, $J=275.4$ Hz), 79.59, 41.30 - 31.76 (br, 2C), 38.05 (br), 36.29 (br), 35.32, 33.71, 31.14 (q, $J=3.2 \mathrm{~Hz}), 31.12$ (q, $J=31.1 \mathrm{~Hz}), 28.56$.

${ }^{19}$ F NMR (282 MHz, CDCl $) \delta-73.67(\mathrm{~d}, J=9.5 \mathrm{~Hz}, 3 \mathrm{~F})$.

Data are consistent with those reported in the literature: Kautzky, J. A.; Wang, T.; Evans, R. W.; MacMillan, D. W. C. J. Am. Chem. Soc. 2018, 140, 6522. 


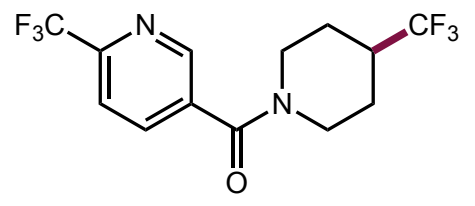

\section{(4-(trifluoromethyl)piperidin-1-yl)(6-(trifluoromethyl)pyridin-3-yl)methanone (33)}

Prepared following the general procedure outlined above using $\operatorname{Ir}\left[(\mathrm{F}-\mathrm{mppy})_{2}(\mathrm{phen})\right] \mathrm{PF}_{6}(2.2 \mathrm{mg}$, $2.5 \mu \mathrm{mol}, 0.005$ equiv.), $\mathrm{CuBr}$ ( $14.3 \mathrm{mg}, 0.1 \mathrm{mmol}, 0.20$ equiv.), 2,2'-bipyridine ( $20 \mathrm{mg}, 0.125$ mmol, 0.25 equiv.), AdNHSi(TMS) 3 (400 mg, 2.0 mmol, 2.0 equiv.), NaOAc (164 mg, 2.0 mmol, 4.0 equiv.), (4-bromopiperidin-1-yl)(6-(trifluoromethyl)pyridin-3-yl)methanone (168 mg, 0.5 mmol, 1.0 equiv.), $\mathrm{TMSCF}_{3}$ reagent $(220 \mu \mathrm{L}, 1.5 \mathrm{mmol}, 3.0$ equiv.) and $\mathrm{MeCN}(3.0 \mathrm{~mL}, 0.17 \mathrm{M})$.

The final reaction mixture was diluted with $\mathrm{MeCN}(20 \mathrm{~mL})$ and then filtered over celite. After washing the celite with $\mathrm{MeCN}$ three times, the organic solution was concentrated to yield the crude product as an oil. The product was purified via silica gel column chromatography (gradient 1 to $10 \%$ acetone in toluene) to yield the pure product ( $110 \mathrm{mg}, 68 \%$ yield).

${ }^{1}$ H NMR (500 MHz, CDCl $) \delta 8.76(\mathrm{~d}, J=2.1 \mathrm{~Hz}, 1 \mathrm{H}), 7.94(\mathrm{dd}, J=8.0,2.1 \mathrm{~Hz}, 1 \mathrm{H}), 7.77(\mathrm{~d}, J$ $=8.0 \mathrm{~Hz}, 1 \mathrm{H}), 4.84(\mathrm{br}, 1 \mathrm{H}), 3.76(\mathrm{br}, 1 \mathrm{H}), 3.13(\mathrm{br}, 1 \mathrm{H}), 2.62(\mathrm{br}, 1 \mathrm{H}), 2.41-2.29(\mathrm{~m}, 1 \mathrm{H})$, $2.12-1.87(\mathrm{~m}, 2 \mathrm{H}), 1.72-1.47(\mathrm{~m}, 2 \mathrm{H})$.

${ }^{13}$ C NMR (125 MHz, $\left.\mathbf{C D C l}_{3}\right) \delta$ 166.54, 149.28 (q, $\left.J=35.2 \mathrm{~Hz}\right), 148.05,136.56,134.36,126.82$ (q, $J=278.3 \mathrm{~Hz}), 121.23$ (q, $J=274.7 \mathrm{~Hz}), 120.68$ (q, $J=2.7 \mathrm{~Hz}), 46.73$ (br), 41.38 (br), 40.54 (q, $J=27.9 \mathrm{~Hz}$ ), 25.41 (br), 24.30 (br).

${ }^{19}$ F NMR (282 MHz, CDCl $) \delta-68.17(\mathrm{~s}, 3 \mathrm{~F}),-73.81(\mathrm{~d}, J=8.0 \mathrm{~Hz}, 3 \mathrm{~F})$.

IR (film) $\mathbf{v}_{\max } 2930,2857,1632,1444,1334,1283,1175,1125,1082,986,856 \mathrm{~cm}^{-1}$.

HRMS (ESI-TOF) m/z calcd. for $\mathrm{C}_{13} \mathrm{H}_{13} \mathrm{~F}_{6} \mathrm{~N}_{2}\left([\mathrm{M}+\mathrm{H}]^{+}\right)$327.0927, found 327.0932. 
b) General procedures for pentafluoroethylation: To an oven-dried 40-mL vial equipped with a cross shaped stir bar were added AdNHSi(TMS) 3 (0.9 mmol, $358 \mathrm{mg}, 1.8$ equiv.), NaOAc (2.0 mmol, $164 \mathrm{mg}, 4.0$ equiv.), substrate ( $0.5 \mathrm{mmol}, 1.0$ equiv.), $\mathrm{CuBr}$ ( $0.1 \mathrm{mmol}, 14.3 \mathrm{mg}, 0.2$ equiv.), ligand (0.125 mmol, 0.25 equiv.) and photocatalyst $\operatorname{Ir}\left[(\text { ppy })_{2}(\mathrm{bpy})\right] \mathrm{PF}_{6}(2.0 \mathrm{mg}, 2.5 \mu \mathrm{mol}, 0.005$ equiv.). Then $2.5 \mathrm{~mL} \mathrm{MeCN}$ was added via syringe, followed by addition of $\mathrm{TMSC}_{2} \mathrm{~F}_{5}(260 \mu \mathrm{L}$, $1.5 \mathrm{mmol}, 3.0$ equiv.). After the reaction mixture was stirred for $5 \mathrm{~min}$, the vial septum was pierced by an $18 \mathrm{G}$ needle, which was left in the septum for the duration of the reaction to allow for air flow without solvent evaporation. The reaction vial was then placed in the Integrated Photoreactor for $16 \mathrm{~h}$ under the following conditions: 45\% LED intensity, $500 \mathrm{rpm}$ stirring and $5200 \mathrm{rpm}$ fan speed. Work up and purification for each substrate are described below. 


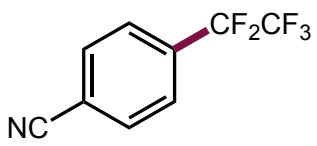

\section{4-(perfluoroethyl)benzonitrile (34)}

Prepared following the general procedure outlined above using $\operatorname{Ir}\left[(\mathrm{ppy})_{2}(\mathrm{bpy})\right] \mathrm{PF}_{6}(2.0 \mathrm{mg}, 2.5$ $\mu$ mol, 0.005 equiv.), $\mathrm{CuBr}$ (14.3 mg, $0.1 \mathrm{mmol}, 0.20$ equiv.), 4,4'-dimethyl-2,2'-bipyridine (23 mg, 0.125 mmol, 0.25 equiv.), AdNHSi(TMS) 3 (358 mg, 0.9 mmol, 1.8 equiv.), NaOAc (164 mg, 2.0 mmol, 4.0 equiv.), 4-bromobenzonitrile ( $91.0 \mathrm{mg}, 0.5 \mathrm{mmol}, 1.0$ equiv.), $\mathrm{TMSC}_{2} \mathrm{~F}_{5}$ reagent (260 $\mu \mathrm{L}, 1.5 \mathrm{mmol}, 3.0$ equiv.) and $\mathrm{MeCN}(2.5 \mathrm{~mL}, 0.2 \mathrm{M})$.

The final reaction mixture was diluted with EtOAc $(20 \mathrm{~mL})$ and then filtered over celite. After washing the celite with EtOAc three times, the organic solution was concentrated to yield the crude product as an oil. The product was purified via silica gel column chromatography (gradient 1 to $20 \%$ DCM in pentane) to yield the pure product (92 $\mathrm{mg}, 84 \%$ yield).

${ }^{1}$ H NMR (400 MHz, $\left.\mathbf{C D C l}_{3}\right) \delta 7.83(\mathrm{~d}, J=8.8 \mathrm{~Hz}, 2 \mathrm{H}), 7.74(\mathrm{~d}, J=8.3 \mathrm{~Hz}, 2 \mathrm{H})$.

${ }^{13}$ C NMR (125 MHz, $\left.\mathbf{C D C l}_{3}\right) \delta 133.17(\mathrm{t}, J=24.4 \mathrm{~Hz}), 132.72,127.57(\mathrm{td}, J=6.3,1.2 \mathrm{~Hz})$, $118.83(\mathrm{dt}, J=286.1,38.4 \mathrm{~Hz}), 117.54,116.41(\mathrm{t}, J=1.8 \mathrm{~Hz}), 112.74(\mathrm{tq}, J=255.1,38.7 \mathrm{~Hz})$.

${ }^{19}$ F NMR (282 MHz, CDCl $) \delta-84.51(\mathrm{~s}, 3 \mathrm{~F}),-115.75(\mathrm{~s}, 2 \mathrm{~F})$.

Data are consistent with those reported in the literature: Li, L. C.; Ni, C. F.; Xie, Q. Q.; Hu, M. Y.; Wang, F.; Hu, J. B. Angew. Chem. Int. Ed. 2017, 56, 9971. 


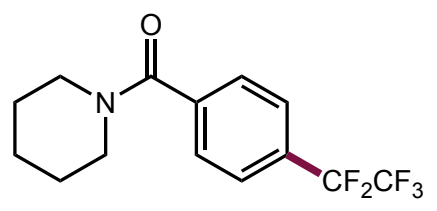

(4-(perfluoroethyl)phenyl)(piperidin-1-yl)methanone (35)

Prepared following the general procedure outlined above using $\operatorname{Ir}\left[(\mathrm{ppy})_{2}(\mathrm{bpy})\right] \mathrm{PF}_{6}(2.0 \mathrm{mg}, 2.5$ $\mu$ mol, 0.005 equiv.), $\mathrm{CuBr}$ (14.3 mg, $0.1 \mathrm{mmol}, 0.20$ equiv.), 4,4'-dimethyl-2,2'-bipyridine (23 mg, 0.125 mmol, 0.25 equiv.), AdNHSi(TMS) 3 (358 mg, 0.9 mmol, 1.8 equiv.), NaOAc (164 mg, 2.0 mmol, 4.0 equiv.), (4-bromophenyl)(piperidin-1-yl)methanone (134 mg, 0.5 mmol, 1.0 equiv.), $\mathrm{TMSC}_{2} \mathrm{~F}_{5}$ reagent $(260 \mu \mathrm{L}, 1.5 \mathrm{mmol}, 3.0$ equiv.) and $\mathrm{MeCN}(2.5 \mathrm{~mL}, 0.2 \mathrm{M})$.

The final reaction mixture was diluted with $\mathrm{MeCN}(20 \mathrm{~mL})$ and then filtered over celite. After washing the celite with MeCN three times, the organic solution was concentrated to yield the crude product as an oil. The product was purified via silica gel column chromatography (gradient 5 to $20 \% \mathrm{Et}_{2} \mathrm{O}$ in DCM) to yield the pure product (114 $\mathrm{mg}, 75 \%$ yield).

${ }^{1}$ H NMR (400 MHz, CDCl$)_{3} \delta 7.64(\mathrm{~d}, J=8.1 \mathrm{~Hz}, 2 \mathrm{H}), 7.51(\mathrm{~d}, J=8.5 \mathrm{~Hz}, 2 \mathrm{H}), 3.73$ (br, 2H), 3.31 (br, 2H), 1.70 (br, 4H), 1.53 (br, 2H).

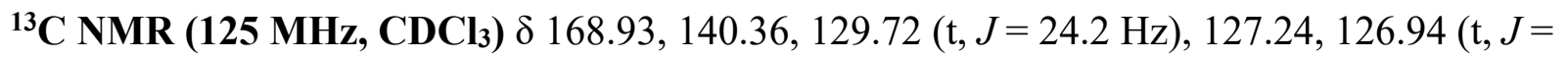
$6.3 \mathrm{~Hz}), 119.10$ (qt, $J=286.0,39.1 \mathrm{~Hz}), 113.27$ (tq, $J=254.5,38.3 \mathrm{~Hz}), 48.82$ (br), 43.30 (br), 26.69 (br), 25.70 (br), 24.62.

${ }^{19}$ F NMR (376 MHz, CDCl 3$) \delta-84.65(\mathrm{~s}, 3 \mathrm{~F}),-115.00(\mathrm{~s}, 2 \mathrm{~F})$.

IR (film) $v_{\max } 2802,1655,1472,1345,1290,1217,1150,1084,1005,1001,963,864,735 \mathrm{~cm}^{-1}$.

HRMS (ESI-TOF) m/z calcd. for $\mathrm{C}_{14} \mathrm{H}_{15} \mathrm{~F}_{5} \mathrm{NO}\left([\mathrm{M}+\mathrm{H}]^{+}\right)$308.1068, found 308.1069. 


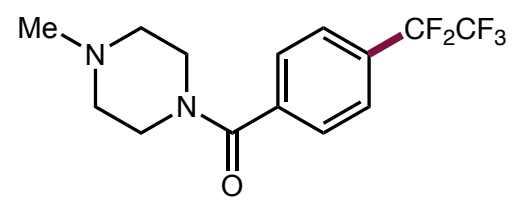

\section{(4-methylpiperazin-1-yl)(4-(perfluoroethyl)phenyl)methanone (36)}

Prepared following the general procedure outlined above using $\operatorname{Ir}\left[(\mathrm{ppy})_{2}(\mathrm{bpy})\right] \mathrm{PF}_{6}(2.0 \mathrm{mg}, 2.5$ $\mu$ mol, 0.005 equiv.), $\mathrm{CuBr}$ (14.3 mg, $0.1 \mathrm{mmol}, 0.20$ equiv.), 4,4'-dimethyl-2,2'-bipyridine (23 mg, 0.125 mmol, 0.25 equiv.), AdNHSi(TMS) 3 (358 mg, 0.9 mmol, 1.8 equiv.), NaOAc (164 mg, 2.0 mmol, 4.0 equiv.), (4-bromophenyl)(4-methylpiperazin-1-yl)methanone (142 mg, $0.5 \mathrm{mmol}, 1.0$ equiv.), $\mathrm{TMSC}_{2} \mathrm{~F}_{5}$ reagent $(260 \mu \mathrm{L}, 1.5 \mathrm{mmol}, 3.0$ equiv.) and $\mathrm{MeCN}(2.5 \mathrm{~mL}, 0.2 \mathrm{M})$.

The final reaction mixture was diluted with $\mathrm{MeCN}(20 \mathrm{~mL})$ and then filtered over celite. After washing the celite with $\mathrm{MeCN}$ three times, the organic solution was concentrated to yield the crude product as an oil. The product was purified via silica gel column chromatography (gradient 1 to $5 \% \mathrm{MeOH}$ in DCM) to yield the pure product (104 mg, 65\% yield).

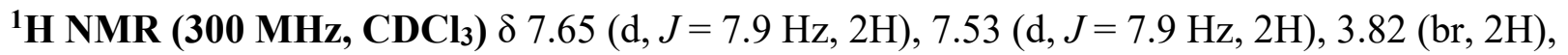
3.40 (br, 2H), 2.51 (br, 2H), 2.36 (br, 2H), 2.33 (s, 3H).

${ }^{13}$ C NMR (125 MHz, $\left.\mathbf{C D C l}_{3}\right) \delta 168.89,139.57,131.85,130.03(\mathrm{t}, J=24.1 \mathrm{~Hz}), 128.91,127.47$, $126.99(\mathrm{t}, J=6.2 \mathrm{~Hz}), 119.04(\mathrm{qt}, J=285.9,39.1 \mathrm{~Hz}), 112.17(\mathrm{tq}, J=254.2,38.4 \mathrm{~Hz}), 55.26$, $54.65,47.58,46.04,42.11$.

${ }^{19}$ F NMR (282 MHz, $\left.\mathbf{C D C l}_{3}\right) \delta-84.64(\mathrm{~s}, 3 \mathrm{~F}),-115.07(\mathrm{~s}, 2 \mathrm{~F})$.

IR (film) $v_{\max } 2797,1635,1436,1340,1288,1204,1146,1094,1018,1001,973,844,720 \mathrm{~cm}^{-1}$.

HRMS (ESI-TOF) m/z calcd. for $\mathrm{C}_{14} \mathrm{H}_{15} \mathrm{~F}_{5} \mathrm{~N}_{2} \mathrm{O}\left([\mathrm{M}+\mathrm{H}]^{+}\right) 323.1177$, found 323.1178 . 


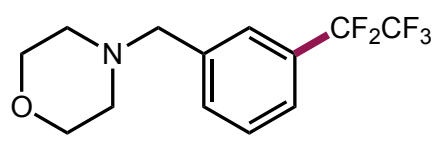

\section{4-(3-(perfluoroethyl)benzyl)morpholine (37)}

Prepared following the general procedure outlined above using $\operatorname{Ir}\left[(\mathrm{ppy})_{2}(\mathrm{bpy})\right] \mathrm{PF}_{6}(2.0 \mathrm{mg}, 2.5$ $\mu \mathrm{mol}, 0.005$ equiv.), $\mathrm{CuBr}$ (14.3 mg, $0.1 \mathrm{mmol}, 0.20$ equiv.), 4,4'-dimethyl-2,2'-bipyridine ( $23 \mathrm{mg}$, $0.125 \mathrm{mmol}, 0.25$ equiv.), AdNHSi(TMS) 3 (358 mg, 0.9 mmol, 1.8 equiv.), NaOAc (164 mg, 2.0 mmol, 4.0 equiv.), 4-(3-bromobenzyl)morpholine (128 mg, $100 \mu \mathrm{L}, 0.5 \mathrm{mmol}, 1.0$ equiv.), $\mathrm{TMSC}_{2} \mathrm{~F}_{5}$ reagent $(260 \mu \mathrm{L}, 1.5 \mathrm{mmol}, 3.0$ equiv.) and $\mathrm{MeCN}(2.5 \mathrm{~mL}, 0.2 \mathrm{M})$.

The final reaction mixture was diluted with $\mathrm{MeCN}(20 \mathrm{~mL})$ and then filtered over celite. After washing the celite with $\mathrm{MeCN}$ three times, the organic solution was concentrated to yield the crude product as an oil. The product was purified via silica gel column chromatography (gradient 1 to $5 \%$ acetone in DCM) to yield the pure product (103 $\mathrm{mg}, 70 \%$ yield).

${ }^{1} \mathbf{H}$ NMR (500 MHz, $\left.\mathbf{C D C l}_{3}\right) \delta 7.57(\mathrm{~s}, 1 \mathrm{H}), 7.55(\mathrm{~d}, J=7.4 \mathrm{~Hz}, 1 \mathrm{H}), 7.50-7.43(\mathrm{~m}, 2 \mathrm{H}), 3.71$ (t, $J=4.6 \mathrm{~Hz}, 4 \mathrm{H}), 3.55(\mathrm{~s}, 2 \mathrm{H}), 2.44(\mathrm{t}, J=4.6 \mathrm{~Hz}, 4 \mathrm{H})$.

${ }^{13}$ C NMR (125 MHz, $\left.\mathbf{C D C l}_{3}\right) \delta$ 139.15, $132.64(\mathrm{~d}, J=1.7 \mathrm{~Hz}), 128.91(\mathrm{t}, 23.9 \mathrm{~Hz}), 128.85$, $126.97(\mathrm{t}, J=6.3 \mathrm{~Hz}), 125.38(\mathrm{t}, J=6.3 \mathrm{~Hz}), 119.25(\mathrm{qt}, J=285.9,39.5 \mathrm{~Hz}), 113.57$ (tq, $J=$ 254.5, 38.1 Hz), 67.10, 62.94, 53.69 .

${ }^{19}$ F NMR (282 MHz, CDCl 3$) ~ \delta-84.75(\mathrm{t}, J=2.8 \mathrm{~Hz}, 3 \mathrm{~F}),-114.74(\mathrm{~s}, 2 \mathrm{~F})$.

IR (film) $v_{\max } 2811,1447,1336,1293,1203,1141,1117,1084,1007,914,868,809,787,750$, $705 \mathrm{~cm}^{-1}$.

HRMS (ESI-TOF) $\mathrm{m} / \mathrm{z}$ calcd. for $\mathrm{C}_{13} \mathrm{H}_{15} \mathrm{~F}_{5} \mathrm{NO}\left([\mathrm{M}+\mathrm{H}]^{+}\right)$296.1068, found 296.1064 . 


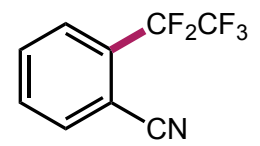

\section{2-(perfluoroethyl)benzonitrile (38)}

Prepared following the general procedure outlined above using $\operatorname{Ir}\left[(\mathrm{ppy})_{2}(\mathrm{bpy})\right] \mathrm{PF}_{6}(2.0 \mathrm{mg}, 2.5$ $\mu$ mol, 0.005 equiv.), $\mathrm{CuBr}$ (14.3 mg, $0.1 \mathrm{mmol}, 0.20$ equiv.), 4,4'-dimethyl-2,2'-bipyridine (23 mg, 0.125 mmol, 0.25 equiv.), AdNHSi(TMS) 3 (358 mg, 0.9 mmol, 1.8 equiv.), NaOAc (164 mg, 2.0 mmol, 4.0 equiv.), 2-bromobenzonitrile ( $92 \mathrm{mg}, 0.5 \mathrm{mmol}, 1.0$ equiv.), $\mathrm{TMSC}_{2} \mathrm{~F}_{5}$ reagent (260 $\mu \mathrm{L}$, $1.5 \mathrm{mmol}, 3.0$ equiv.) and $\mathrm{MeCN}(2.5 \mathrm{~mL}, 0.2 \mathrm{M})$.

The final reaction mixture was diluted with $\mathrm{Et}_{2} \mathrm{O}(20 \mathrm{~mL})$ and then filtered over celite. After washing the celite with $\mathrm{Et}_{2} \mathrm{O}$ three times, the organic solution was concentrated to yield the crude product as an oil. The product was purified via silica gel column chromatography (gradient 5 to $20 \%$ DCM in pentane) to yield the pure product (77 $\mathrm{mg}, 70 \%$ yield).

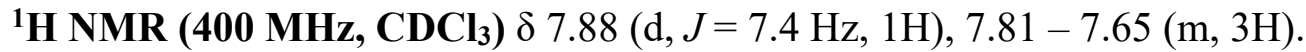

${ }^{13}$ C NMR (125 MHz, CDCl 3$) \delta 135.41,133.01,132.56(\mathrm{~d}, J=1.4 \mathrm{~Hz}), 130.95(\mathrm{t}, J=23.7 \mathrm{~Hz})$, $128.82(\mathrm{t}, J=7.3 \mathrm{~Hz}), 118.91$ (qt, $J=286.7,38.2 \mathrm{~Hz}), 115.86,112.59$ (tq, $J=256.9,39.4 \mathrm{~Hz}$ ), $111.47(\mathrm{t}, J=3.7 \mathrm{~Hz})$.

${ }^{19}$ F NMR (376 MHz, CDCl 3$) \delta-84.07(\mathrm{t}, J=3.5 \mathrm{~Hz}, 3 \mathrm{~F}),-112.70(\mathrm{~s}, 2 \mathrm{~F})$.

IR (film) $v_{\max } 1448,1334,1294,1264,1206,1157,1118,1080,1048,974,960,843,766,749$, $661 \mathrm{~cm}^{-1}$.

HRMS (GC-EI-TOF) m/z calcd. for $\mathrm{C}_{9} \mathrm{H}_{4} \mathrm{~F}_{5} \mathrm{~N}\left(\left[\mathrm{M}^{*}\right]^{+}\right)$221.0258, found 221.0254 . 


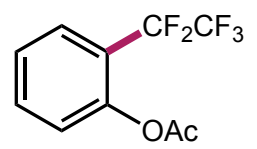

\section{2-(perfluoroethyl)phenyl acetate (39)}

Prepared following the general procedure outlined above using $\operatorname{Ir}\left[(\mathrm{ppy})_{2}(\mathrm{bpy})\right] \mathrm{PF}_{6} \quad(2.0 \mathrm{mg}, 2.5$ $\mu$ mol, 0.005 equiv.), $\mathrm{CuBr}$ (14.3 mg, $0.1 \mathrm{mmol}, 0.20$ equiv.), 4,4'-dimethyl-2,2'-bipyridine (23 mg, 0.125 mmol, 0.25 equiv.), AdNHSi(TMS) 3 (358 mg, 0.9 mmol, 1.8 equiv.), NaOAc (164 mg, 2.0 mmol, 4.0 equiv.), 2-bromophenyl acetate (108 mg, $75 \mu 1,0.5 \mathrm{mmol}, 1.0$ equiv.), $\mathrm{TMSC}_{2} \mathrm{~F}_{5}$ reagent (260 $\mu \mathrm{L}, 1.5 \mathrm{mmol}, 3.0$ equiv.) and $\mathrm{MeCN}(2.5 \mathrm{~mL}, 0.2 \mathrm{M})$.

The final reaction mixture was diluted with $\mathrm{Et}_{2} \mathrm{O}(20 \mathrm{~mL})$ and then filtered over celite. After washing the celite with $\mathrm{Et}_{2} \mathrm{O}$ three times, the organic solution was concentrated to yield the crude product as an oil. The product was purified via silica gel column chromatography (gradient 1 to $10 \%$ acetone in DCM) to yield the pure product ( $82 \mathrm{mg}, 65 \%$ yield).

${ }^{1}$ H NMR (500 MHz, CDCl $) \delta 7.67-7.56(\mathrm{~m}, 2 \mathrm{H}), 7.37(\mathrm{t}, J=7.7 \mathrm{~Hz}, 1 \mathrm{H}), 7.22(\mathrm{dd}, J=8.2$, $1.1 \mathrm{~Hz}, 1 \mathrm{H}), 2.31(\mathrm{~s}, 3 \mathrm{H})$.

${ }^{13}$ C NMR (125 MHz, CDCl $) \delta 169.06,148.81(\mathrm{~d}, J=3.1 \mathrm{~Hz}), 133.33(\mathrm{t}, J=1.4 \mathrm{~Hz}), 128.82(\mathrm{t}$, $J=7.9 \mathrm{~Hz}), 126.29,125.02,120.85(\mathrm{t}, J=23.0 \mathrm{~Hz}), 119.20(\mathrm{qt}, J=286.5,38.7 \mathrm{~Hz}), 113.19(\mathrm{tq}$, $J=255.3,39.8 \mathrm{~Hz}), 20.85$.

${ }^{19}$ F NMR (376 MHz, CDCl 3$) \delta-84.34(\mathrm{t}, J=2.8 \mathrm{~Hz}, 3 \mathrm{~F}),-112.82(\mathrm{~s}, 2 \mathrm{~F})$.

IR (film) $v_{\max } 1753,1625,1507,1487,1435,1367,1300,1275,746,701,669 \mathrm{~cm}^{-1}$.

HRMS (GC-EI-TOF) m/z calcd. for $\mathrm{C}_{9} \mathrm{H}_{4} \mathrm{~F}_{5} \mathrm{~N}\left([\mathrm{M}+\mathrm{H}-\mathrm{Ac}]^{+}\right)$212.0255, found 212.0259. 


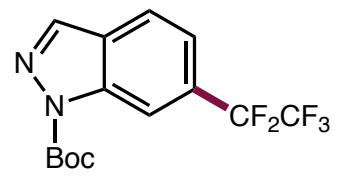

tert-butyl 6-(perfluoroethyl)-1H-indazole-1-carboxylate (40)

Prepared following the general procedure outlined above using $\operatorname{Ir}\left[(\mathrm{ppy})_{2}(\mathrm{bpy})\right] \mathrm{PF}_{6}(2.0 \mathrm{mg}, 2.5$ $\mu \mathrm{mol}, 0.005$ equiv.), $\mathrm{CuBr}$ (14.3 mg, $0.1 \mathrm{mmol}, 0.20$ equiv.), 4,4'-dimethyl-2,2'-bipyridine (23 mg, 0.125 mmol, 0.25 equiv.), AdNHSi(TMS) 3 (358 mg, 0.9 mmol, 1.8 equiv.), NaOAc (164 mg, 2.0 mmol, 4.0 equiv.), tert-butyl 6-bromo- $1 H$-indazole-1-carboxylate (150 mg, $0.5 \mathrm{mmol}, 1.0$ equiv.), $\mathrm{TMSC}_{2} \mathrm{~F}_{5}$ reagent $(260 \mu \mathrm{L}, 1.5 \mathrm{mmol}, 3.0$ equiv.) and $\mathrm{MeCN}(2.5 \mathrm{~mL}, 0.2 \mathrm{M})$.

The final reaction mixture was diluted with EtOAc $(20 \mathrm{~mL})$ and then filtered over celite. After washing the celite with EtOAc three times, the organic solution was concentrated to yield the crude product as an oil. The product was purified via silica gel column chromatography (gradient 1 to $15 \%$ DCM in hexane) to yield the pure product as a white crystalline solid (117 $\mathrm{mg}, 70 \%$ yield).

${ }^{1}$ H NMR (500 MHz, $\left.\mathbf{C D C l}_{3}\right) \delta 8.53(\mathrm{~s}, 1 \mathrm{H}), 8.26(\mathrm{~s}, 1 \mathrm{H}), 7.88(\mathrm{~d}, J=8.4 \mathrm{~Hz}, 1 \mathrm{H}), 7.54(\mathrm{~d}, J=$ $8.4 \mathrm{~Hz}, 1 \mathrm{H}), 1.74(\mathrm{~s}, 9 \mathrm{H})$.

${ }^{13}$ C NMR (125 MHz, $\left.\mathbf{C D C l}_{3}\right) \delta$ 148.85, 139.19, 139.16, 129.26 (t, $\left.J=23.7 \mathrm{~Hz}\right), 128.00,121.87$, $121.44(\mathrm{t}, J=5.9 \mathrm{~Hz}), 119.19(\mathrm{qt}, J=286.1,39.2 \mathrm{~Hz}), 113.96(\mathrm{t}, J=7.6 \mathrm{~Hz}), 113.65(\mathrm{tq}, J=$ 254.5, 38.2 Hz), 85.97, 28.24.

${ }^{19}$ F NMR (376 MHz, CDCl 3$) \delta-84.37(\mathrm{~s}, 3 \mathrm{~F}),-113.55(\mathrm{~s}, 2 \mathrm{~F})$.

IR (film) $v_{\max } 2984,1742,1487,1415,1372,1352,1302,1198,1138,1089,1032,994,913$, $887,846,815,785,762,744,698,665 \mathrm{~cm}^{-1}$.

HRMS (ESI-TOF) m/z calcd. for $\mathrm{C}_{9} \mathrm{H}_{6} \mathrm{~F}_{5} \mathrm{~N}_{2}\left([\mathrm{M}+2 \mathrm{H}-\mathrm{Boc}]^{+}\right)$237.0446, found 237.0448. 


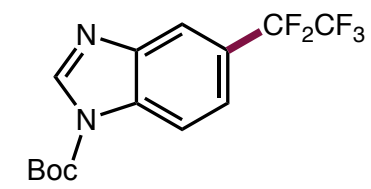

tert-butyl 5-(perfluoroethyl)-1 $H$-benzo[d]imidazole-1-carboxylate (41)

Prepared following the general procedure outlined above using $\operatorname{Ir}\left[(\mathrm{ppy})_{2}(\mathrm{bpy})\right] \mathrm{PF}_{6}(2.0 \mathrm{mg}, 2.5$ $\mu$ mol, 0.005 equiv.), $\mathrm{CuBr}$ (14.3 mg, $0.1 \mathrm{mmol}, 0.20$ equiv.), 4,4'-dimethyl-2,2'-bipyridine (23 mg, 0.125 mmol, 0.25 equiv.), AdNHSi(TMS) 3 (358 mg, 0.9 mmol, 1.8 equiv.), NaOAc (164 mg, 2.0 mmol, 4.0 equiv.), tert-butyl 5-bromo- $1 H$-benzo[d]imidazole-1-carboxylate $(150 \mathrm{mg}, 0.5 \mathrm{mmol}$, 1.0 equiv.), $\mathrm{TMSC}_{2} \mathrm{~F}_{5}$ reagent $(260 \mu \mathrm{L}, 1.5 \mathrm{mmol}, 3.0$ equiv.) and $\mathrm{MeCN}(2.5 \mathrm{~mL}, 0.2 \mathrm{M})$.

The final reaction mixture was diluted with EtOAc $(20 \mathrm{~mL})$ and then filtered over celite. After washing the celite with EtOAc three times, the organic solution was concentrated to yield the crude product as an oil. The product was purified via silica gel column chromatography (gradient 10 to $50 \%$ DCM in hexane) to yield the pure product as a white crystalline solid (98 $\mathrm{mg}$, 68\% yield).

${ }^{1}$ H NMR (500 MHz, DMSO-d 6 ) $\delta 8.86(\mathrm{~s}, 1 \mathrm{H}), 8.17$ (d, $\left.J=8.6 \mathrm{~Hz}, 1 \mathrm{H}\right), 8.10$ (s, 1H), 7.73 (d, $J$ $=8.6 \mathrm{~Hz}, 1 \mathrm{H}), 1.66(\mathrm{~s}, 9 \mathrm{H})$.

${ }^{13}$ C NMR (125 MHz, DMSO-d 6 ) $\delta$ 147.19, 145.17, 143.64, 133.79, 122.78 (t, $\left.J=6.3 \mathrm{~Hz}\right)$, $122.73(\mathrm{t}, J=23.9 \mathrm{~Hz}), 119.86(\mathrm{t}, J=287.3,40.4 \mathrm{~Hz}), 118.71(\mathrm{t}, J=6.7 \mathrm{~Hz}), 115.34,113.57(\mathrm{dq}$, $J=253.6,37.8 \mathrm{~Hz}), 86.20,27.53$.

${ }^{19}$ F NMR (282 MHz, DMSO-d $) \delta-84.02(\mathrm{~s}, 3 \mathrm{~F}),-112.03(\mathrm{~s}, 2 \mathrm{~F})$

IR (film) $v_{\max } 2985,1753,1625,1507,1487,1435,1367,1300,1275,1232,1198,1147,1080$, $1062,1036,993,902,890,842,818,806,769,755,746,701,669 \mathrm{~cm}^{-1}$.

HRMS (ESI-TOF) m/z calcd. for $\mathrm{C}_{9} \mathrm{H}_{6} \mathrm{~F}_{5} \mathrm{~N}_{2}\left([\mathrm{M}+2 \mathrm{H}-\mathrm{Boc}]^{+}\right)$237.0446, found 237.0450. 


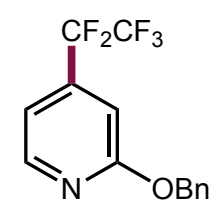

\section{2-(benzyloxy)-4-(perfluoroethyl)pyridine (42)}

Prepared following the general procedure outlined above using $\operatorname{Ir}\left[(\mathrm{ppy})_{2}(\mathrm{bpy}) \mathrm{PF}_{6} \quad(2.0 \mathrm{mg}, 2.5\right.$ $\mu \mathrm{mol}, 0.005$ equiv.), $\mathrm{CuBr}$ ( $14.3 \mathrm{mg}, 0.1 \mathrm{mmol}, 0.20$ equiv.), 4,4'-dimethyl-2,2'-bipyridine ( $23 \mathrm{mg}$, $0.125 \mathrm{mmol}, 0.25$ equiv.), AdNHSi(TMS) 3 (358 mg, $0.9 \mathrm{mmol}, 1.8$ equiv.), NaOAc (164 mg, 2.0 mmol, 4.0 equiv.), 2-(benzyloxy)-4-bromopyridine ( $132 \mathrm{mg}, 0.5 \mathrm{mmol}, 1.0$ equiv.), $\mathrm{TMSC}_{2} \mathrm{~F}_{5}$ reagent (260 $\mu \mathrm{L}, 1.5 \mathrm{mmol}, 3.0$ equiv.) and $\mathrm{MeCN}(2.5 \mathrm{~mL}, 0.2 \mathrm{M})$.

The final reaction mixture was diluted with EtOAc $(20 \mathrm{~mL})$ and then filtered over celite. After washing the celite with EtOAc three times, the organic solution was concentrated to yield the crude product as an oil. The product was purified via silica gel column chromatography (gradient 5 to $25 \%$ DCM in hexane) to yield the pure product as a white crystalline solid (124mg, $82 \%$ yield).

${ }^{1}$ H NMR (500 MHz, $\left.\mathbf{C D C l}_{3}\right) \delta 8.35(\mathrm{~d}, J=5.3 \mathrm{~Hz}, 1 \mathrm{H}), 7.48(\mathrm{~d}, J=7.5 \mathrm{~Hz}, 2 \mathrm{H}), 7.43-7.33$ (m, 3H), $7.08(\mathrm{~d}, J=5.4 \mathrm{~Hz}, 1 \mathrm{H}), 7.05(\mathrm{~s}, 1 \mathrm{H}), 5.44(\mathrm{~s}, 2 \mathrm{H})$.

${ }^{13}$ C NMR (125 MHz, CDCl $) \delta$ ) 164.08, 148.23, 139.77 (t, $\left.J=24.9 \mathrm{~Hz}\right), 136.73,128.69,128.26$, 128.24, 118.84 (qt, $J=286.2,37.9 \mathrm{~Hz}), 113.59$ (t, $J=5.6 \mathrm{~Hz}$ ), 111.31 (tq, $J=254.6,38.7 \mathrm{~Hz}$ ), $109.51(\mathrm{t}, J=6.6 \mathrm{~Hz}), 68.45$.

${ }^{19}$ F NMR (282 MHz, CDCl $) \delta-84.48$ (s, 3F), -116.85 (s, 2F).

Data are consistent with those reported in the literature: Mormino, M. G.; Fier, P. S.; Hartwig, J. F. Org. Lett. 2014, 16, 1744. 


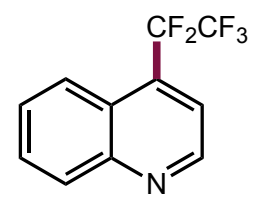

\section{4-(perfluoroethyl)quinoline (43)}

Prepared following the general procedure outlined above using $\operatorname{Ir}\left[(\mathrm{ppy})_{2}(\mathrm{bpy}) \mathrm{PF}_{6} \quad(2.0 \mathrm{mg}, 2.5\right.$ $\mu \mathrm{mol}, 0.005$ equiv.), $\mathrm{CuBr}$ (14.3 mg, $0.1 \mathrm{mmol}, 0.20$ equiv.), 4,4'-dimethyl-2,2'-bipyridine ( $23 \mathrm{mg}$, $0.125 \mathrm{mmol}, 0.25$ equiv.), AdNHSi(TMS) 3 (358 mg, $0.9 \mathrm{mmol}, 1.8$ equiv.), NaOAc (164 mg, 2.0 mmol, 4.0 equiv.), 4-bromoquinoline (104 $\mathrm{mg}, 0.5 \mathrm{mmol}, 1.0$ equiv.), $\mathrm{TMSC}_{2} \mathrm{~F}_{5}$ reagent ( $260 \mu \mathrm{L}$, $1.5 \mathrm{mmol}, 3.0$ equiv.) and $\mathrm{MeCN}(2.5 \mathrm{~mL}, 0.2 \mathrm{M})$.

The final reaction mixture was diluted with EtOAc $(20 \mathrm{~mL})$ and then filtered over celite. After washing the celite with EtOAc three times, the organic solution was concentrated to yield the crude product as an oil. The product was purified via silica gel column chromatography (gradient 10 to $50 \%$ DCM in hexane) to yield the pure product as a white crystalline solid ( $86 \mathrm{mg}, 70 \%$ yield).

${ }^{1} \mathbf{H}$ NMR (400 MHz, $\left.\mathbf{C D C l}_{3}\right) \delta 9.06(\mathrm{dd}, J=4.5,1.0 \mathrm{~Hz}, 1 \mathrm{H}), 8.26-8.22(\mathrm{~m}, 1 \mathrm{H}), 8.20(\mathrm{~d}, J=$ $9.0 \mathrm{~Hz}, 1 \mathrm{H}), 7.82(\mathrm{~m}, 1 \mathrm{H}), 7.72-7.64(\mathrm{~m}, 2 \mathrm{H})$.

${ }^{13}$ C NMR (100 MHz, CDCl $)$ ) $\delta$ 149.42, 149.27, 133.23 (t, $\left.J=22.8 \mathrm{~Hz}\right), 130.84,130.28,128.48$, 124.65 (t, $J=5.7$ ), 124.07, 120.65 (t, $J=8.4 \mathrm{~Hz}$ ), 119.63 (qt, $J=287.8,37.6 \mathrm{~Hz}$ ), 114.26 (tq, $J=$ 257.6, 39.7 Hz).

${ }^{19}$ F NMR (376 MHz, CDCl $) \delta-83.24$ (s, 3F), -110.55 (s, 2F).

Data are consistent with those reported in the literature: Nagase, M.; Kuninobu, Y.; Kanai, M. J. Am. Chem. Soc. 2016, 138, 6103. 


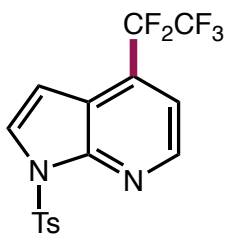

\section{4-(perfluoroethyl)-1-tosyl-1H-pyrrolo[2,3-b]pyridine (44)}

Prepared following the general procedure outlined above using $\operatorname{Ir}\left[(\mathrm{ppy})_{2}(\mathrm{bpy})\right] \mathrm{PF}_{6}(2.0 \mathrm{mg}, 2.5$ $\mu$ mol, 0.005 equiv.), $\mathrm{CuBr}$ (14.3 mg, $0.1 \mathrm{mmol}, 0.20$ equiv.), 4,4'-dimethyl-2,2'-bipyridine (23 mg, 0.125 mmol, 0.25 equiv.), AdNHSi(TMS) 3 (358 mg, 0.9 mmol, 1.8 equiv.), NaOAc (164 mg, 2.0 mmol, 4.0 equiv.), 4-bromo-1-tosyl-1H-pyrrolo[2,3-b]pyridine (176 mg, $0.5 \mathrm{mmol}, 1.0$ equiv.), $\mathrm{TMSC}_{2} \mathrm{~F}_{5}$ reagent $(260 \mu \mathrm{L}, 1.5 \mathrm{mmol}, 3.0$ equiv.) and $\mathrm{MeCN}(2.5 \mathrm{~mL}, 0.2 \mathrm{M})$.

The final reaction mixture was diluted with EtOAc $(20 \mathrm{~mL})$ and then filtered over celite. After washing the celite with EtOAc three times, the organic solution was concentrated to yield the crude product as an oil. The product was purified via silica gel column chromatography (gradient 10 to $50 \%$ DCM in hexane) to yield the pure product as a white crystalline solid (123 $\mathrm{mg}$, 63\% yield).

${ }^{1}$ H NMR (500 MHz, $\left.\mathbf{C D C l}_{3}\right) \delta 8.56(\mathrm{~d}, J=5.0 \mathrm{~Hz}, 1 \mathrm{H}), 8.10(\mathrm{~d}, J=8.4 \mathrm{~Hz}, 2 \mathrm{H}), 7.90(\mathrm{~d}, J=$ $4.1 \mathrm{~Hz}, 1 \mathrm{H}), 7.38(\mathrm{~d}, J=5.1 \mathrm{~Hz}, 1 \mathrm{H}), 7.31(\mathrm{~d}, J=8.1 \mathrm{~Hz}, 2 \mathrm{H}), 6.74(\mathrm{dd}, J=4.3,2.2 \mathrm{~Hz}, 1 \mathrm{H})$, $2.39(\mathrm{~s}, 3 \mathrm{H})$.

${ }^{13}$ C NMR (125 MHz, $\left.\mathbf{C D C l}_{3}\right) \delta 147.69,145.88,144.81,135.02,129.96,129.50(\mathrm{t}, J=25.5 \mathrm{~Hz})$, $128.72(\mathrm{~d}, J=1.6 \mathrm{~Hz}), 128.48,120.07$ (t, $J=3.6 \mathrm{~Hz}), 119.06(\mathrm{qt}, J=287.3,38.0 \mathrm{~Hz}), 116.46$ (t, $J=6.6 \mathrm{~Hz}), 113.25(\mathrm{tq}, J=255.8,39.4 \mathrm{~Hz}), 104.03(\mathrm{t}, J=3.1 \mathrm{~Hz}), 21.85$.

${ }^{19}$ F NMR (376 MHz, CDCl $) \delta-84.25(\mathrm{~s}, J=2.1 \mathrm{~Hz}, 3 \mathrm{~F}),-114.90(\mathrm{~d}, J=2.6 \mathrm{~Hz}, 2 \mathrm{~F})$.

IR (film) $v_{\max } 1595,1518,1371,1331,1292,1265,1210,1192,1174,1163,1142,1107,1089$, $1040,985,899,838,813,793,731,687,672,658 \mathrm{~cm}^{-1}$.

HRMS (ESI-TOF) m/z calcd. for $\mathrm{C}_{16} \mathrm{H}_{12} \mathrm{~F}_{5} \mathrm{~N}_{2} \mathrm{O}_{2} \mathrm{~S}\left([\mathrm{M}+\mathrm{H}]^{+}\right)$391.0534, found 391.0536. 


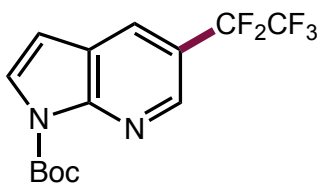

tert-butyl 5-(perfluoroethyl)-1 $H$-pyrrolo[2,3-b]pyridine-1-carboxylate (45)

Prepared following the general procedure outlined above using $\operatorname{Ir}\left[(\mathrm{ppy})_{2}(\mathrm{bpy})\right] \mathrm{PF}_{6}(2.0 \mathrm{mg}, 2.5$ $\mu$ mol, 0.005 equiv.), $\mathrm{CuBr}$ (14.3 mg, $0.1 \mathrm{mmol}, 0.20$ equiv.), 4,4'-dimethyl-2,2'-bipyridine (23 mg, 0.125 mmol, 0.25 equiv.), AdNHSi(TMS) 3 (358 mg, 0.9 mmol, 1.8 equiv.), NaOAc (164 mg, 2.0 mmol, 4.0 equiv.), tert-butyl 5-bromo-1H-pyrrolo[2,3-b]pyridine-1-carboxylate (150 mg, 0.5 mmol, 1.0 equiv.), $\mathrm{TMSC}_{2} \mathrm{~F}_{5}$ reagent $(260 \mu \mathrm{L}, 1.5 \mathrm{mmol}, 3.0$ equiv.) and $\mathrm{MeCN}(2.5 \mathrm{~mL}, 0.2 \mathrm{M})$.

The final reaction mixture was diluted with EtOAc $(20 \mathrm{~mL})$ and then filtered over celite. After washing the celite with EtOAc three times, the organic solution was concentrated to yield the crude product as an oil. The product was purified via silica gel column chromatography (gradient 5 to $35 \%$ DCM in hexane) to yield the pure product as a crystalline solid (106 $\mathrm{mg}, 64 \%$ yield).

${ }^{1}$ H NMR (500 MHz, $\left.\mathbf{C D C l}_{3}\right) \delta 8.71(\mathrm{~d}, J=2.2 \mathrm{~Hz}, 1 \mathrm{H}), 8.11(\mathrm{~d}, J=2.2 \mathrm{~Hz}, 1 \mathrm{H}), 7.78(\mathrm{~d}, J=$ $4.1 \mathrm{~Hz}, 1 \mathrm{H}), 6.61(\mathrm{~d}, J=4.1 \mathrm{~Hz}, 1 \mathrm{H}), 1.69(\mathrm{~s}, 9 \mathrm{H})$.

${ }^{13}$ C NMR (125 MHz, CDCl $) \delta 149.78(\mathrm{t}, J=1.7 \mathrm{~Hz}), 147.68,143.04(\mathrm{t}, J=6.3 \mathrm{~Hz}), 128.82$, $127.85(\mathrm{t}, J=6.3 \mathrm{~Hz}), 122.53,119.76(\mathrm{t}, J=24.3 \mathrm{~Hz}), 119.13(\mathrm{qt}, J=286.0,39.5 \mathrm{~Hz}), 113.61$ (tq, $J=254.5,38.8 \mathrm{~Hz}), 104.69,85.19,28.19$.

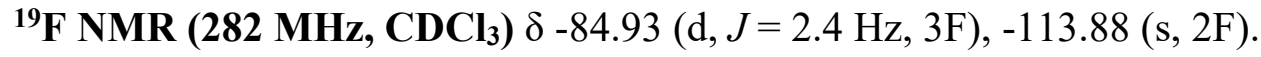

IR (film) $v_{\max } 2926,1739,1611,1572,1531,1480,1395,1372,1315,1274,1242,1201,1152$, $1097,1087,1010,917,898,790,768,735,696,661 \mathrm{~cm}^{-1}$.

HRMS (ESI-TOF) m/z calcd. for $\mathrm{C}_{9} \mathrm{H}_{6} \mathrm{~F}_{5} \mathrm{~N}_{2}\left([\mathrm{M}+2 \mathrm{H}-\mathrm{Boc}]^{+}\right)$237.0446, found 237.0444 . 


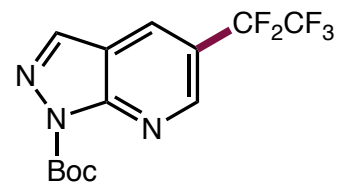

tert-butyl 5-(perfluoroethyl)-1 $H$-pyrazolo[3,4-b]pyridine-1-carboxylate (46)

Prepared following the general procedure outlined above using $\operatorname{Ir}\left[(\mathrm{ppy})_{2}(\mathrm{bpy})\right] \mathrm{PF}_{6}(2.0 \mathrm{mg}, 2.5$ $\mu$ mol, 0.005 equiv.), $\mathrm{CuBr}$ (14.3 mg, $0.1 \mathrm{mmol}, 0.20$ equiv.), 4,4'-dimethyl-2,2'-bipyridine (23 mg, 0.125 mmol, 0.25 equiv.), AdNHSi(TMS) 3 (358 mg, 0.9 mmol, 1.8 equiv.), NaOAc (164 mg, 2.0 mmol, 4.0 equiv.), tert-butyl 5-bromo-1H-pyrazolo[3,4-b]pyridine-1-carboxylate (150 mg, 0.5 mmol, 1.0 equiv.), $\mathrm{TMSC}_{2} \mathrm{~F}_{5}$ reagent $(260 \mu \mathrm{L}, 1.5 \mathrm{mmol}, 3.0$ equiv.) and $\mathrm{MeCN}(2.5 \mathrm{~mL}, 0.2 \mathrm{M})$.

The final reaction mixture was diluted with EtOAc $(20 \mathrm{~mL})$ and then filtered over celite. After washing the celite with EtOAc three times, the organic solution was concentrated to yield the crude product as an oil. The product was purified via silica gel column chromatography ( $1 \%$ acetone in DCM) to yield the pure product as a white crystalline solid (105 mg, 63\% yield).

${ }^{1}$ H NMR (500 MHz, CDCl $) \delta 8.95(\mathrm{~d}, J=2.2 \mathrm{~Hz}, 1 \mathrm{H}), 8.37(\mathrm{~d}, J=2.2 \mathrm{~Hz}, 1 \mathrm{H}), 8.30(\mathrm{~s}, 1 \mathrm{H})$, $1.74(\mathrm{~s}, 9 \mathrm{H})$.

${ }^{13}$ C NMR (125 MHz, $\left.\mathbf{C D C l}_{3}\right) \delta 153.08,148.51(\mathrm{td}, J=6.3,1.5 \mathrm{~Hz}), 147.45,137.54,129.76(\mathrm{t}, J$ $=6.4 \mathrm{~Hz}), 120.84(\mathrm{t}, J=24.6 \mathrm{~Hz}), 118.94(\mathrm{qt}, J=285.9,38.9 \mathrm{~Hz}), 117.13,113.17(\mathrm{tq}, J=255.8$ $39.1 \mathrm{~Hz}), 86.51,28.17$.

${ }^{19}$ F NMR (376 MHz, CDCl 3$) \delta-84.81(\mathrm{~s}, 3 \mathrm{~F}),-113.86(\mathrm{~s}, 2 \mathrm{~F})$.

IR (film) $v_{\max } 1760,1395,1372,1334,1286,1245,1208,1183,1150,1094,1053,1012,911$, $847,779,734,702 \mathrm{~cm}^{-1}$.

HRMS (ESI-TOF) m/z calcd. for $\mathrm{C}_{8} \mathrm{H}_{5} \mathrm{~F}_{5} \mathrm{~N}_{3}\left([\mathrm{M}+2 \mathrm{H}-\mathrm{Boc}]^{+}\right)$238.0398, found 238.0400. 


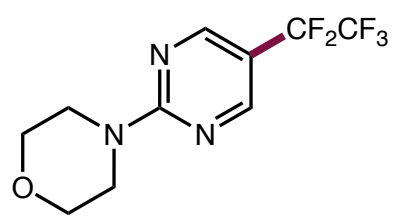

\section{4-(5-(perfluoroethyl)pyrimidin-2-yl)morpholine (47)}

Prepared following the general procedure outlined above using $\operatorname{Ir}\left[(\mathrm{ppy})_{2}(\mathrm{bpy})\right] \mathrm{PF}_{6} \quad(2.0 \mathrm{mg}, 2.5$ $\mu \mathrm{mol}, 0.005$ equiv.), $\mathrm{CuBr}$ (14.3 mg, $0.1 \mathrm{mmol}, 0.20$ equiv.), 4,4'-dimethyl-2,2'-bipyridine (23 mg, 0.125 mmol, 0.25 equiv.), AdNHSi(TMS) 3 (358 mg, 0.9 mmol, 1.8 equiv.), NaOAc (164 mg, 2.0 mmol, 4.0 equiv), 4-(5-bromopyrimidin-2-yl)morpholine (122 mg, $0.5 \mathrm{mmol}, 1.0$ equiv.), $\mathrm{TMSC}_{2} \mathrm{~F}_{5}$ reagent $(260 \mu \mathrm{L}, 1.5 \mathrm{mmol}, 3.0$ equiv.) and $\mathrm{MeCN}(2.5 \mathrm{~mL}, 0.2 \mathrm{M})$.

The final reaction mixture was diluted with EtOAc $(20 \mathrm{~mL})$ and then filtered over celite. After washing the celite with EtOAc three times, the organic solution was concentrated to yield the crude product as an oil. The product was purified via silica gel column chromatography (gradient 1 to $5 \%$ EA in DCM) to yield the pure product ( $87 \mathrm{mg}, 62 \%$ yield).

${ }^{1}$ H NMR (400 MHz, CDCl $) \delta 8.45(\mathrm{~s}, 2 \mathrm{H}), 3.89-3.91(\mathrm{~m}, 4 \mathrm{H}), 3.76-3.78(\mathrm{~m}, 4 \mathrm{H})$.

${ }^{13}$ C NMR (125 MHz, $\left.\mathbf{C D C l}_{3}\right) \delta 162.38,156.37$ (t, $\left.J=6.3 \mathrm{~Hz}\right), 119.06$ (qt, $\left.J=286.0,40.3 \mathrm{~Hz}\right)$, $113.20(\mathrm{tq}, J=254.5,39.1 \mathrm{~Hz}), 111.01(\mathrm{t}, J=25.2 \mathrm{~Hz}), 66.84,44.36$.

${ }^{19}$ F NMR (376 MHz, CDCl $) \delta-85.53(\mathrm{t}, J=2.6 \mathrm{~Hz}, 3 \mathrm{~F}),-114.98(\mathrm{~s}, J=2.7 \mathrm{~Hz}, 2 \mathrm{~F})$.

IR (film) $v_{\max } 2863,1614,1540,1447,1300,1260,1214,1111,1098,1038,949,846,798,739$, $656 \mathrm{~cm}^{-1}$.

HRMS (ESI-TOF) m/z calcd. for $\mathrm{C}_{10} \mathrm{H}_{11} \mathrm{~F}_{5} \mathrm{~N}_{3} \mathrm{O}\left([\mathrm{M}+\mathrm{H}]^{+}\right)$284.0817, found 284.0813. 


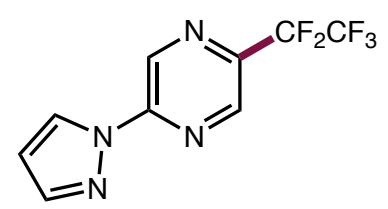

\section{2-(1H-pyrazol-1-yl)-5-(perfluoroethyl)pyrazine (48)}

Prepared following the general procedure outlined above using $\operatorname{Ir}\left[(\mathrm{ppy})_{2}(\mathrm{bpy})\right] \mathrm{PF}_{6} \quad(2.0 \mathrm{mg}, 2.5$ $\mu$ mol, 0.005 equiv.), $\mathrm{CuBr}$ ( $14.3 \mathrm{mg}, 0.1 \mathrm{mmol}, 0.20$ equiv.), 4,4'-dimethyl-2,2'-bipyridine ( $23 \mathrm{mg}$, $0.125 \mathrm{mmol}, 0.25$ equiv.), AdNHSi(TMS) 3 (358 mg, 0.9 mmol, 1.8 equiv.), NaOAc (164 mg, 2.0 mmol, 4.0 equiv.), 2-bromo-5-(1H-pyrazol-1yl)pyrazine (113 mg, $0.5 \mathrm{mmol}, 1.0$ equiv.), $\mathrm{TMSC}_{2} \mathrm{~F}_{5}$ reagent $(260 \mu \mathrm{L}, 1.5 \mathrm{mmol}, 3.0$ equiv. $)$ and $\mathrm{MeCN}(2.5 \mathrm{~mL}, 0.2 \mathrm{M})$.

The final reaction mixture was diluted with EtOAc $(20 \mathrm{~mL})$ and then filtered over celite. After washing the celite with EtOAc three times, the organic solution was concentrated to yield the crude

product as an oil. The product was purified via silica gel column chromatography (gradient 5 to $25 \%$ DCM in pentane) to yield the pure product as a crystalline solid ( $86 \mathrm{mg}, 65 \%$ yield).

${ }^{1}$ H NMR (300 MHz, CDCl $) \delta 9.44(\mathrm{~d}, J=1.4 \mathrm{~Hz}, 1 \mathrm{H}), 8.71(\mathrm{~d}, J=1.4 \mathrm{~Hz}, 1 \mathrm{H}), 8.55(\mathrm{~d}, J=$ $2.7 \mathrm{~Hz}, 1 \mathrm{H}), 7.85$ (d, $J=1.6 \mathrm{~Hz}, 1 \mathrm{H}), 6.56$ (dd, $J=2.7,1.7 \mathrm{~Hz}, 1 \mathrm{H})$.

${ }^{13}$ C NMR (125 MHz, CDCl $) \delta$ 148.97, 144.47, 140.95 (t, $\left.J=5.3 \mathrm{~Hz}\right), 139.90$ (t, $\left.J=26.5 \mathrm{~Hz}\right)$, 135.44, 128.24, 118.81 (qt, $J=286.6,37.4 \mathrm{~Hz}), 111.15$ (tq, $J=255.5,38.7 \mathrm{~Hz}$ ), 109.82.

${ }^{19} \mathbf{F}$ NMR (282 MHz, $\left.\mathbf{C D C l}_{3}\right) \delta-83.41(\mathrm{t}, J=2.0 \mathrm{~Hz}, 3 \mathrm{~F}),-117.48(\mathrm{q}, J=2.3 \mathrm{~Hz}, 2 \mathrm{~F})$.

IR (film) $v_{\max } 2916,1591,1548,1529,1499,1436,1398,1331,1305,1237,1190,1146,1128$, $1104,1042,1020,984,937,912,844,774,739 \mathrm{~cm}^{-1}$.

HRMS (GC-EI-TOF) $\mathrm{m} / \mathrm{z}$ calcd. for $\mathrm{C}_{9} \mathrm{H}_{5} \mathrm{~F}_{5} \mathrm{~N}_{4}\left(\left[\mathrm{M}^{*}\right]^{+}\right)$264.0429, found 264.0432. 


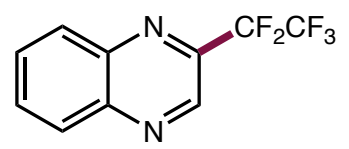

\section{2-(perfluoroethyl)quinoxaline (49)}

Prepared following the general procedure outlined above using $\operatorname{Ir}\left[(\mathrm{ppy})_{2}(\mathrm{bpy})\right] \mathrm{PF}_{6}(2.0 \mathrm{mg}, 2.5$ $\mu$ mol, 0.005 equiv.), $\mathrm{CuBr}$ (14.3 mg, $0.1 \mathrm{mmol}, 0.20$ equiv.), 4,4'-dimethyl-2,2'-bipyridine (23 mg, 0.125 mmol, 0.25 equiv.), AdNHSi(TMS) 3 (358 mg, 0.9 mmol, 1.8 equiv.), NaOAc (164 mg, 2.0 mmol, 4.0 equiv), 2-bromoquinoxaline ( $105 \mathrm{mg}, 0.5 \mathrm{mmol}, 1.0$ equiv.), $\mathrm{TMSC}_{2} \mathrm{~F}_{5}$ reagent ( $260 \mu \mathrm{L}$, $1.5 \mathrm{mmol}, 3.0$ equiv.) and $\mathrm{MeCN}(2.5 \mathrm{~mL}, 0.2 \mathrm{M})$.

The final reaction mixture was diluted with EtOAc $(20 \mathrm{~mL})$ and then filtered over celite. After washing the celite with EtOAc three times, the organic solution was concentrated to yield the crude product as an oil. The product was purified via silica gel column chromatography (gradient 10 to $50 \%$ DCM in pentane) to yield the pure product as a white crystalline solid (82 $\mathrm{mg}, 66 \%$ yield).

${ }^{1}$ H NMR (500 MHz, $\left.\mathbf{C D C l}_{3}\right) \delta 9.18(\mathrm{~s}, 1 \mathrm{H}), 8.24(\mathrm{ddd}, J=12.1,8.0,2.0 \mathrm{~Hz}, 2 \mathrm{H}), 7.97$ - 7.90 $(\mathrm{m}, 2 \mathrm{H})$.

${ }^{13}$ C NMR (125 MHz, CDCl $) \delta 143.69$ (t, $\left.J=1.4 \mathrm{~Hz}\right), 142.64(\mathrm{t}, J=25.9 \mathrm{~Hz}), 141.85(\mathrm{t}, J=4.2$ $\mathrm{Hz}), 141.24,132.68,131.70,130.34,129.66,118.87$ (qt, $J=286.8,36.8 \mathrm{~Hz}), 111.22(\mathrm{tq}, J=$ $256.3,38.6 \mathrm{~Hz})$.

${ }^{19}$ F NMR (376 MHz, CDCl 3$) \delta-82.89$ (s, 3F), -116.96 (s, 2F).

IR (film) $v_{\max } 2863,1614,1540,1447,1300,1260,1214,1111,1098,1038,949,846,798,739$, $656 \mathrm{~cm}^{-1}$.

Data are consistent with those reported in the literature: Huang, Y. J.; Ajitha, M. J.; Huang, K. W.; Zhang, Z. X; Weng, Z. Q. Dalton Trans. 2016. 45. 8468. 


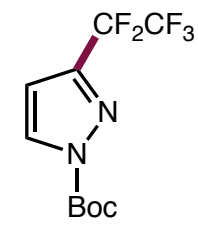

tert-butyl 3-(perfluoroethyl)-1H-pyrazole-1-carboxylate (50)

Prepared following the general procedure outlined above using $\operatorname{Ir}\left[(\mathrm{ppy})_{2}(\mathrm{bpy})\right] \mathrm{PF}_{6}(2.0 \mathrm{mg}, 2.5$ $\mu \mathrm{mol}, 0.005$ equiv.), $\mathrm{CuBr}$ (14.3 mg, $0.1 \mathrm{mmol}, 0.20$ equiv.), 4,4'-dimethyl-2,2'-bipyridine (23 mg, 0.125 mmol, 0.25 equiv.), AdNHSi(TMS) 3 (358 mg, 0.9 mmol, 1.8 equiv.), NaOAc (164 mg, 2.0 mmol, 4.0 equiv.), tert-butyl 3-bromo-1H-pyrazole-1-carboxylate (125 mg, 0.5 mmol, 1.0 equiv.), $\mathrm{TMSC}_{2} \mathrm{~F}_{5}$ reagent $(260 \mu \mathrm{L}, 1.5 \mathrm{mmol}, 3.0$ equiv.) and $\mathrm{MeCN}(2.5 \mathrm{~mL}, 0.2 \mathrm{M})$.

***Y Yeld was determined by ${ }^{19} \mathrm{~F}$ NMR in duplicate due to the high volatility of the desired product. The desired product was isolated for full characterizations. ${ }^{* *}$

1,4-difluorobenzene $\left(52 \mu \mathrm{L}, 0.5 \mathrm{mmol}, 1.0\right.$ equiv.) was added as the internal standard for ${ }^{19} \mathrm{~F} \mathrm{NMR}$ analysis (DMSO- $\left.d_{6}\right)$. ( $50 \%$ yield - average of two trials: $49 \%$ yield, $51 \%$ yield).

The final reaction mixture was diluted with $\mathrm{Et}_{2} \mathrm{O}(20 \mathrm{~mL})$ and then filtered over celite. After washing the celite with $\mathrm{Et}_{2} \mathrm{O}$ three times, the organic solution was concentrated to yield the crude product as an oil. The product was purified via silica gel column chromatography (gradient 10 to $50 \%$ DCM in pentane) to yield the pure product.

${ }^{1}$ H NMR (500 MHz, $\left.\mathbf{C D C l}_{3}\right) \delta 8.16(\mathrm{~d}, J=2.9 \mathrm{~Hz}, 1 \mathrm{H}), 6.64(\mathrm{~d}, J=2.8 \mathrm{~Hz}, 1 \mathrm{H}), 1.66(\mathrm{~s}, 9 \mathrm{H})$.

${ }^{13}$ C NMR (125 MHz, CDCl 3$) ~ \delta 146.56,145.28$ (t, $\left.J=28.8 \mathrm{~Hz}\right), 132.40,118.75$ (qt, $J=286.0$, $37.1 \mathrm{~Hz}), 110.30$ (tq, $J=252.4,39.6 \mathrm{~Hz}), 107.61$ (td, $J=3.1,1.5 \mathrm{~Hz}), 87.22,27.94$.

${ }^{19}$ F NMR (376 MHz, CDCl 3$) \delta-84.28(\mathrm{t}, J=2.7 \mathrm{~Hz}, 3 \mathrm{~F}),-113.95(\mathrm{~s}, 2 \mathrm{~F})$.

IR (film) $v_{\max } 1758,1478,1380,1275,1235,1180,1130,1100,1048,964,948,780,745 \mathrm{~cm}^{-1}$.

HRMS (ESI-TOF) m/z calcd. for $\mathrm{C}_{5} \mathrm{H}_{4} \mathrm{~F}_{5} \mathrm{~N}_{2}\left([\mathrm{M}+2 \mathrm{H}-\mathrm{Boc}]^{+}\right) 187.0289$, found 187.0286 


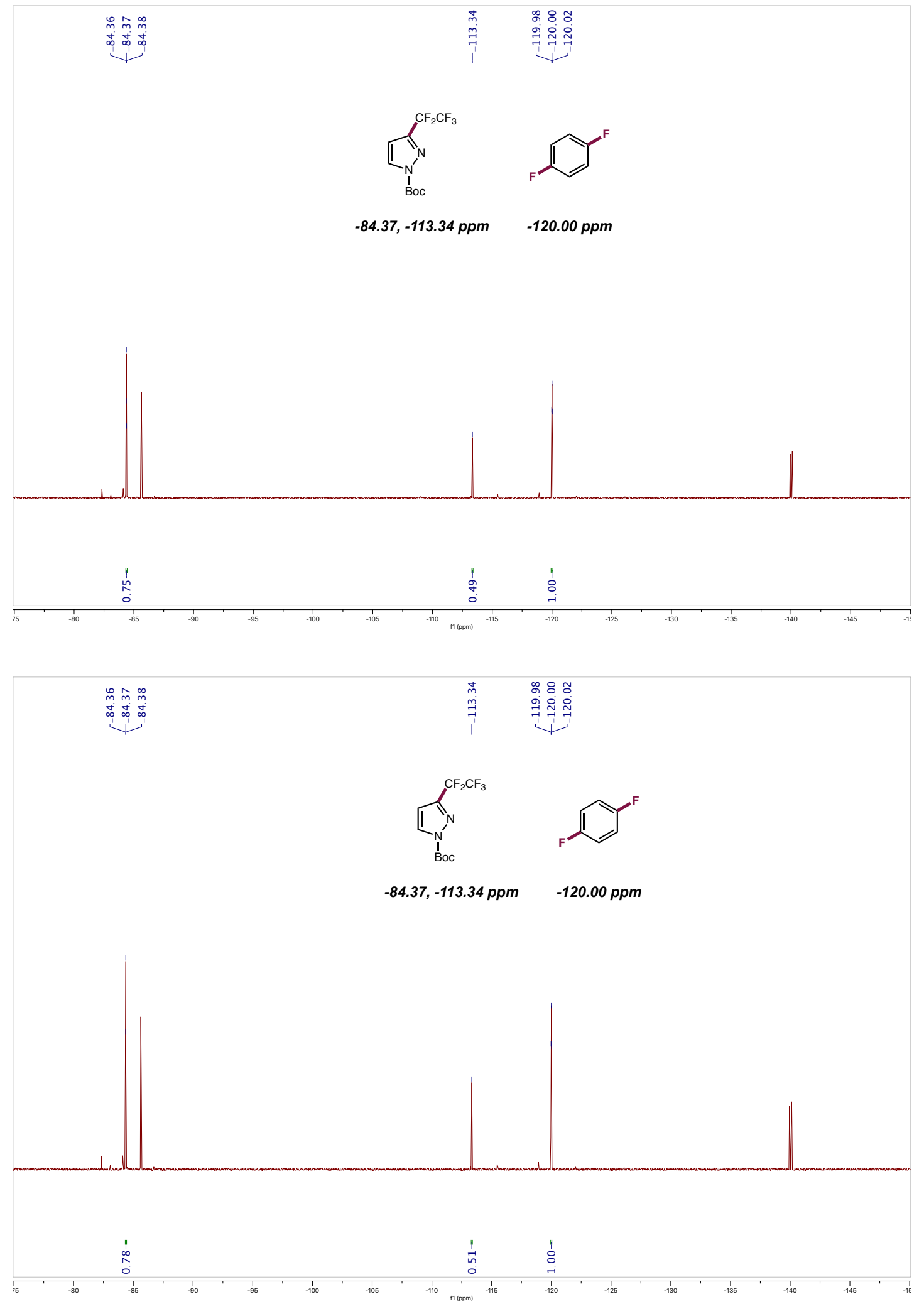

Figure S12. ${ }^{19}$ F NMR assay for tert-butyl 3-(perfluoroethyl)-1H-pyrazole-1-carboxylate (50) 


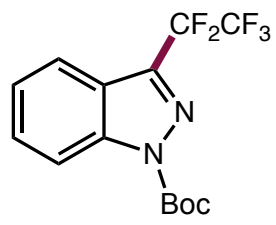

tert-butyl 3-(perfluoroethyl)-1 $H$-indazole-1-carboxylate (51)

Prepared following the general procedure outlined above using $\operatorname{Ir}\left[(\mathrm{ppy})_{2}(\mathrm{bpy})\right] \mathrm{PF}_{6}(2.0 \mathrm{mg}, 2.5$ $\mu$ mol, 0.005 equiv.), $\mathrm{CuBr}$ (14.3 mg, $0.1 \mathrm{mmol}, 0.20$ equiv.), 4,4'-dimethyl-2,2'-bipyridine (23 mg, 0.125 mmol, 0.25 equiv.), AdNHSi(TMS) 3 (358 mg, 0.9 mmol, 1.8 equiv.), NaOAc (164 mg, 2.0 mmol, 4.0 equiv.), tert-butyl 3-bromo- $1 H$-indazole-1-carboxylate (150 mg, $0.5 \mathrm{mmol}, 1.0$ equiv.), $\mathrm{TMSC}_{2} \mathrm{~F}_{5}$ reagent $(260 \mu \mathrm{L}, 1.5 \mathrm{mmol}, 3.0$ equiv.) and $\mathrm{MeCN}(2.5 \mathrm{~mL}, 0.2 \mathrm{M})$.

The final reaction mixture was diluted with EtOAc $(20 \mathrm{~mL})$ and then filtered over celite. After washing the celite with EtOAc three times, the organic solution was concentrated to yield the crude product as an oil. The product was purified via silica gel column chromatography (gradient 5 to $25 \%$ DCM in hexane) to yield the pure product ( $80 \mathrm{mg}, 48 \%$ yield).

${ }^{1}$ H NMR (500 MHz, CDCl$\left.)_{3}\right) \delta 8.21(\mathrm{~d}, J=8.6 \mathrm{~Hz}, 1 \mathrm{H}), 7.84(\mathrm{~d}, J=8.2 \mathrm{~Hz}, 1 \mathrm{H}), 7.61$ (ddd, $J=$ 8.3, 7.0, $1.0 \mathrm{~Hz}, 1 \mathrm{H}), 7.42(\mathrm{t}, J=7.6 \mathrm{~Hz}, 1 \mathrm{H}), 1.74(\mathrm{~s}, 9 \mathrm{H})$.

${ }^{13}$ C NMR (125 MHz, $\left.\mathbf{C D C l}_{3}\right) \delta 148.41,140.84,138.76(\mathrm{t}, J=29.0 \mathrm{~Hz}), 129.88,125.03,122.82$, $120.77,118.90(\mathrm{qt}, J=286.4,37.0 \mathrm{~Hz}), 115.08,111.49$ (tq, $J=253.3,40.0 \mathrm{~Hz}), 86.47,28.20$.

${ }^{19}$ F NMR (376 MHz, CDCl 3$) \delta-83.68(\mathrm{t}, J=2.6 \mathrm{~Hz}, 3 \mathrm{~F}),-113.60$ (q, $\left.J=3.0 \mathrm{~Hz}, 2 \mathrm{~F}\right)$.

IR (film) $v_{\max } 2956,1765,1515,1371,1326,1304,1251,1204,1148,1121,1078,1027,936$, 920, 839, 776, 741, $675 \mathrm{~cm}^{-1}$.

HRMS (ESI-TOF) m/z calcd. for $\mathrm{C}_{9} \mathrm{H}_{6} \mathrm{~F}_{5} \mathrm{~N}_{2}\left([\mathrm{M}+2 \mathrm{H}-\mathrm{Boc}]^{+}\right)$237.0446, found 237.0443 . 


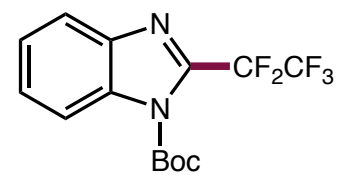

tert-butyl 2-(perfluoroethyl)-1H-benzo[ $[d]$ imidazole-1-carboxylate (52)

Prepared following the general procedure outlined above using $\operatorname{Ir}\left[(\mathrm{ppy})_{2}(\mathrm{bpy})\right] \mathrm{PF}_{6}(2.0 \mathrm{mg}, 2.5$ $\mu \mathrm{mol}, 0.005$ equiv.), $\mathrm{CuBr}$ (14.3 mg, $0.1 \mathrm{mmol}, 0.20$ equiv.), 4,4'-dimethyl-2,2'-bipyridine (23 mg, 0.125 mmol, 0.25 equiv.), AdNHSi(TMS) 3 (358 mg, 0.9 mmol, 1.8 equiv.), NaOAc (164 mg, 2.0 mmol, 4.0 equiv), tert-butyl 2-bromo-1H-benzo[d]imidazole-1-carboxylate (149 mg, $0.5 \mathrm{mmol}$, 1.0 equiv.), $\mathrm{TMSC}_{2} \mathrm{~F}_{5}$ reagent $(260 \mu \mathrm{L}, 1.5 \mathrm{mmol}, 3.0$ equiv.) and $\mathrm{MeCN}(2.5 \mathrm{~mL}, 0.2 \mathrm{M})$.

The final reaction mixture was diluted with EtOAc $(20 \mathrm{~mL})$ and then filtered over celite. After washing the celite with EtOAc three times, the organic solution was concentrated to yield the crude product as an oil. The product was purified via silica gel column chromatography (gradient 2 to $20 \%$ DCM in hexane) to yield the pure product (108 $\mathrm{mg}, 64 \%$ yield).

${ }^{1}$ H NMR (500 MHz, $\left.\mathbf{C D C l}_{3}\right) \delta 8.11(\mathrm{~d}, J=8.3 \mathrm{~Hz}, 1 \mathrm{H}), 7.89(\mathrm{~d}, J=8.1 \mathrm{~Hz}, 1 \mathrm{H}), 7.56-7.49$ (m, 1H), $7.45-7.42(\mathrm{~m}, 1 \mathrm{H}), 1.71(\mathrm{~s}, 9 \mathrm{H})$.

${ }^{13}$ C NMR (125 MHz, $\left.\mathbf{C D C l}_{3}\right) \delta 146.90,140.72,139.64(\mathrm{t}, J=29.6 \mathrm{~Hz}), 133.66,127.83,125.35$, 121.98, 118.89 (qt, $J=286.8,34.9 \mathrm{~Hz}), 115.39,109.48$ (tq, $J=255.2,37.6 \mathrm{~Hz}), 87.51,27.91$.

${ }^{19}$ F NMR (282 MHz, $\left.\mathbf{C D C l}_{3}\right) \delta-80.63(\mathrm{~s}, 3 \mathrm{~F}),-108.72(\mathrm{~s}, 2 \mathrm{~F})$.

IR (film) $v_{\max } 2987,1758,1529,1450,1348,1317,1265,1234,1203,1149,1129,1014,1002$, $906,847,765,704 \mathrm{~cm}^{-1}$.

HRMS (ESI-TOF) m/z calcd. for $\mathrm{C}_{9} \mathrm{H}_{6} \mathrm{~F}_{5} \mathrm{~N}_{2}\left([\mathrm{M}+2 \mathrm{H}-\mathrm{Boc}]^{+}\right)$237.0446, found 237.0445. 


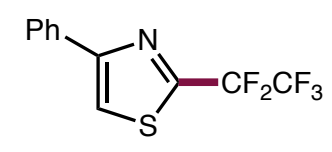

\section{2-(perfluoroethyl)-4-phenylthiazole (53)}

Prepared following the general procedure outlined above using $\operatorname{Ir}\left[(\mathrm{ppy})_{2}(\mathrm{bpy})\right] \mathrm{PF}_{6} \quad(2.0 \mathrm{mg}, 2.5$ $\mu$ mol, 0.005 equiv.), $\mathrm{CuBr}$ ( $14.3 \mathrm{mg}, 0.1 \mathrm{mmol}, 0.20$ equiv.), 4,4'-dimethyl-2,2'-bipyridine ( $23 \mathrm{mg}$, $0.125 \mathrm{mmol}, 0.25$ equiv.), AdNHSi(TMS) 3 (358 mg, $0.9 \mathrm{mmol}, 1.8$ equiv.), $\mathrm{NaOAc}(164 \mathrm{mg}, 2.0$ mmol, 4.0 equiv.), .), 2-bromo-4-phenylthiazole ( $120 \mathrm{mg}, 0.5 \mathrm{mmol}, 1.0$ equiv.), $\mathrm{TMSC}_{2} \mathrm{~F}_{5}$ reagent $(260 \mu \mathrm{L}, 1.5 \mathrm{mmol}, 3.0$ equiv.) and $\mathrm{MeCN}(2.5 \mathrm{~mL}, 0.2 \mathrm{M})$.

The final reaction mixture was diluted with EtOAc $(20 \mathrm{~mL})$ and then filtered over celite. After washing the celite with EtOAc three times, the organic solution was concentrated to yield the crude

product as an oil. The product was purified via silica gel column chromatography (gradient 5 to $15 \%$ DCM in pentane) to yield the pure product as a solid (90 $\mathrm{mg}, 65 \%$ yield).

${ }^{1}$ H NMR (500 MHz, $\left.\mathbf{C D C l}_{3}\right) \delta 7.96-7.92(\mathrm{~m}, 2 \mathrm{H}), 7.75(\mathrm{~s}, 1 \mathrm{H}), 7.50-7.44(\mathrm{~m}, 2 \mathrm{H}), 7.42-$ $7.37(\mathrm{~m}, 1 \mathrm{H})$.

${ }^{13}$ C NMR (125 MHz, $\left.\mathbf{C D C l}_{3}\right) \delta$ 157.73, $155.24(\mathrm{t}, J=30.8 \mathrm{~Hz}), 133.16,129.21,129.09,126.75$, 118.49 (qt, $J=286.6,37.0 \mathrm{~Hz}), 116.41$ (t, $J=1.7 \mathrm{~Hz}), 110.30$ (tq, $J=254.7,39.6 \mathrm{~Hz})$.

${ }^{19}$ F NMR (376 MHz, CDCl $) \delta-83.11(\mathrm{t}, J=2.6 \mathrm{~Hz}, 3 \mathrm{~F}),-108.53(\mathrm{~d}, J=2.7 \mathrm{~Hz}, 2 \mathrm{~F})$.

IR (film) $v_{\max } 1740,1456,1264,1211,1138,1073,1065,703,690,685 \mathrm{~cm}^{-1}$.

HRMS (GC-EI-TOF) $\mathrm{m} / \mathrm{z}$ calcd. for $\mathrm{C}_{11} \mathrm{H}_{6} \mathrm{~F}_{5} \mathrm{NS}\left(\left[\mathrm{M}^{*}\right]^{+}\right)$279.0135, found 279.0130. 


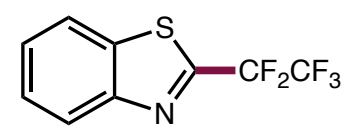

\section{2-(perfluoroethyl)benzo[d]thiazole (54)}

Prepared following the general procedure outlined above using $\operatorname{Ir}\left[(\mathrm{ppy})_{2}(\mathrm{bpy})\right] \mathrm{PF}_{6} \quad(2.0 \mathrm{mg}, 2.5$ $\mu \mathrm{mol}, 0.005$ equiv.), $\mathrm{CuBr}$ (14.3 mg, $0.1 \mathrm{mmol}, 0.20$ equiv.), 4,4'-dimethyl-2,2'-bipyridine ( $23 \mathrm{mg}$, $0.125 \mathrm{mmol}, 0.25$ equiv.), AdNHSi(TMS) 3 (358 mg, 0.9 mmol, 1.8 equiv.), NaOAc (164 mg, 2.0 mmol, 4.0 equiv.), 2-bromobenzo[d]thiazole ( $107 \mathrm{mg}, 0.5 \mathrm{mmol}, 1.0$ equiv.), $\mathrm{TMSC}_{2} \mathrm{~F}_{5}$ reagent $(260 \mu \mathrm{L}, 1.5 \mathrm{mmol}, 3.0$ equiv.) and $\mathrm{MeCN}(2.5 \mathrm{~mL}, 0.2 \mathrm{M})$.

The final reaction mixture was diluted with $\mathrm{Et}_{2} \mathrm{O}(20 \mathrm{~mL})$ and then filtered over celite. After washing the celite with $\mathrm{Et}_{2} \mathrm{O}$ three times, the organic solution was concentrated to yield the crude product as an oil. The product was purified via silica gel column chromatography (gradient 5 to $30 \%$ DCM in pentane) to yield the pure product ( $78 \mathrm{mg}, 62 \%$ yield).

${ }^{1} \mathbf{H}$ NMR (400 MHz, CDCl $) \delta 8.24(\mathrm{~d}, J=8.0 \mathrm{~Hz}, 1 \mathrm{H}), 8.01(\mathrm{dd}, J=7.8,1.5 \mathrm{~Hz}, 1 \mathrm{H}), 7.63(\mathrm{t}, J$ $=7.7 \mathrm{~Hz}, 1 \mathrm{H}), 7.57(\mathrm{t}, J=7.6 \mathrm{~Hz}, 1 \mathrm{H})$.

${ }^{13}$ C NMR (125 MHz, $\left.\mathbf{C D C l}_{3}\right) \delta 155.89$ (t, $\left.J=30.1 \mathrm{~Hz}\right), 152.80,135.35,127.64,127.49,125.23$, $122.10,118.45$ (qt, $J=286.7,36.5 \mathrm{~Hz}), 110.40$ (tq, $J=255.8,39.6 \mathrm{~Hz})$.

${ }^{19}$ F NMR (376 MHz, CDCl $) \delta-82.89(\mathrm{t}, J=2.4 \mathrm{~Hz}, 3 \mathrm{~F}),-109.24(\mathrm{q}, J=2.6 \mathrm{~Hz}, 2 \mathrm{~F})$.

Data are consistent with those reported in the literature: Zhu, J.; Chen, Z.; Xie, H.; Li, S.; Wu, Y. Org. Lett. 2010, 12, 2434. 


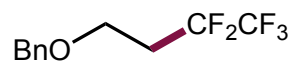

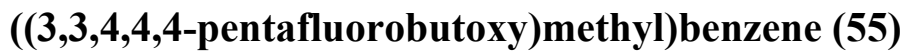

Prepared following the general procedure outlined above using $\operatorname{Ir}\left[(\mathrm{ppy})_{2}(\mathrm{bpy})\right] \mathrm{PF}_{6}(2.0 \mathrm{mg}, 2.5$ $\mu$ mol, 0.005 equiv.), $\mathrm{CuBr}$ (14.3 mg, $0.1 \mathrm{mmol}, 0.20$ equiv.), 2,2'-bipyridine (20 mg, $0.125 \mathrm{mmol}$, 0.25 equiv.), AdNHSi(TMS) 3 (358 mg, 0.9 mmol, 1.8 equiv.), NaOAc (164 mg, $2.0 \mathrm{mmol}, 4.0$ equiv.), benzyl 2-bromoethylether ( $80 \mu \mathrm{L}, 0.5 \mathrm{mmol}, 1.0$ equiv.), $\mathrm{TMSC}_{2} \mathrm{~F}_{5}$ reagent ( $260 \mu \mathrm{L}, 1.5$ mmol, 3.0 equiv.) and $\mathrm{MeCN}(2.5 \mathrm{~mL}, 0.2 \mathrm{M})$.

The final reaction mixture was diluted with $\mathrm{Et}_{2} \mathrm{O}(20 \mathrm{~mL})$ and then filtered over celite. After washing the celite with $\mathrm{Et}_{2} \mathrm{O}$ three times, the organic solution was concentrated to yield the crude

product as an oil. The product was purified via silica gel column chromatography (gradient 1 to $10 \%$ DCM in pentane) to yield the pure product (105 $\mathrm{mg}, 83 \%$ yield).

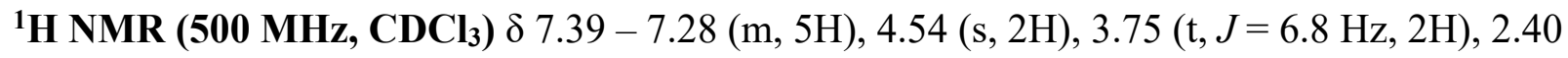
$(\mathrm{ttd}, J=18.1,6.9,1.1 \mathrm{~Hz}, 2 \mathrm{H})$.

${ }^{13}$ C NMR (125 MHz, $\left.\mathbf{C D C l}_{3}\right) \delta 137.70,128.64,128.02,127.80,119.12$ (qt, $\left.J=285.1,35.9 \mathrm{~Hz}\right)$, $115.19(\mathrm{q}, J=253.3,38.2 \mathrm{~Hz}), 73.46,62.25(\mathrm{t}, J=4.5 \mathrm{~Hz}), 31.53(\mathrm{t}, J=21.5 \mathrm{~Hz})$.

${ }^{19}$ F NMR (470 MHz, CDCl 3$) \delta-85.74(\mathrm{~s}, 3 \mathrm{~F}),-117.17(\mathrm{t}, J=18.1 \mathrm{~Hz}, 2 \mathrm{~F})$.

IR (film) $v_{\max } 2917,1455,1364,1190,1107,1034,995,847,736,719,696 \mathrm{~cm}^{-1}$.

HRMS (GC-EI-TOF) m/z calcd. for $\mathrm{C}_{11} \mathrm{H}_{11} \mathrm{~F}_{5} \mathrm{O}\left(\left[\mathrm{M}^{*}\right]^{+}\right)$254.0724, found 254.0718. 


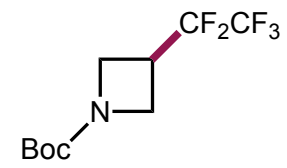

tert-butyl 3-(perfluoroethyl)azetidine-1-carboxylate (56)

Prepared following the general procedure outlined above using $\operatorname{Ir}\left[(\mathrm{ppy})_{2}(\mathrm{bpy})\right] \mathrm{PF}_{6}(2.0 \mathrm{mg}, 2.5$ $\mu$ mol, 0.005 equiv.), $\mathrm{CuBr}$ (14.3 mg, $0.1 \mathrm{mmol}, 0.20$ equiv.), 2,2'-bipyridine (20 mg, $0.125 \mathrm{mmol}$, 0.25 equiv.), AdNHSi(TMS) 3 (358 mg, 0.9 mmol, 1.8 equiv.), NaOAc (164 mg, 2.0 mmol, 4.0 equiv.), 3-bromo-N-Boc-azetidine (118 mg, $0.5 \mathrm{mmol}, 1.0$ equiv.), $\mathrm{TMSC}_{2} \mathrm{~F}_{5}$ reagent $(260 \mu \mathrm{L}, 1.5$ mmol, 3.0 equiv.) and $\mathrm{MeCN}(2.5 \mathrm{~mL}, 0.2 \mathrm{M})$.

The final reaction mixture was diluted with $\mathrm{Et}_{2} \mathrm{O}(20 \mathrm{~mL})$ and then filtered over celite. After washing the celite with $\mathrm{Et}_{2} \mathrm{O}$ three times, the organic solution was concentrated to yield the crude product as an oil. The product was purified via silica gel column chromatography (gradient 15 to $50 \%$ DCM in pentane) to yield the pure product ( $99 \mathrm{mg}, 72 \%$ yield).

${ }^{1}$ H NMR (500 MHz, CDCl $) \delta 4.06(\mathrm{~d}, J=7.6 \mathrm{~Hz}, 4 \mathrm{H}), 3.22(\mathrm{tp}, J=15.1,7.5 \mathrm{~Hz}, 1 \mathrm{H}), 1.44(\mathrm{~s}$, 9H).

${ }^{13}$ C NMR (125 MHz, CDCl 3$) \delta 155.77,119.00$ (qt, $J=285.8,36.3$ Hz), 114.24 (tq, $J=252.1$, $37.7 \mathrm{~Hz}), 80.33,48.04$ (br), 29.37 (t, $J=24.1 \mathrm{~Hz}), 28.30$.

${ }^{19}$ F NMR (282 MHz, CDCl $) \delta-84.43(\mathrm{~s}, 3 \mathrm{~F}),-125.76(\mathrm{~d}, J=15.4 \mathrm{~Hz}, 2 \mathrm{~F})$.

IR (film) $v_{\max } 1706,1368,1335,1199,1159,1034,955,831,696 \mathrm{~cm}^{-1}$.

HRMS (ESI-TOF) m/z calcd. for $\mathrm{C}_{6} \mathrm{H}_{7} \mathrm{~F}_{5} \mathrm{NO}\left([\mathrm{M}+2 \mathrm{H}-(t-\mathrm{Bu})]^{+}\right) 220.0392$, found 220.0391 . 


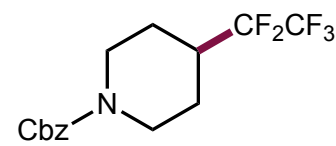

\section{benzyl 4-(perfluoroethyl)piperidine-1-carboxylate (57)}

Prepared following the general procedure outlined above using $\operatorname{Ir}\left[(\mathrm{ppy})_{2}(\mathrm{bpy})\right] \mathrm{PF}_{6}(2.0 \mathrm{mg}, 2.5$ $\mu$ mol, 0.005 equiv.), $\mathrm{CuBr}$ (14.3 mg, $0.1 \mathrm{mmol}, 0.20$ equiv.), 2,2'-bipyridine (20 mg, $0.125 \mathrm{mmol}$, 0.25 equiv.), AdNHSi(TMS) 3 (358 mg, 0.9 mmol, 1.8 equiv.), NaOAc (164 mg, 2.0 mmol, 4.0 equiv.), 4-bromo-N-carboxybenzoylpiperidine ( $149 \mathrm{mg}, 0.5 \mathrm{mmol}, 1.0$ equiv.), $\mathrm{TMSC}_{2} \mathrm{~F}_{5}$ reagent (260 $\mu \mathrm{L}, 1.5 \mathrm{mmol}, 3.0$ equiv.) and $\mathrm{MeCN}(2.5 \mathrm{~mL}, 0.2 \mathrm{M})$.

The final reaction mixture was diluted with EtOAc $(20 \mathrm{~mL})$ and then filtered over celite. After washing the celite with EtOAc three times, the organic solution was concentrated to yield the crude product as an oil. The product was purified via silica gel column chromatography (gradient 10 to $40 \%$ DCM in pentane) to yield the pure product ( $75 \mathrm{mg}, 45 \%$ yield).

${ }^{1}$ H NMR (400 MHz, CDCl 3 ) $\delta 7.42$ - 7.27 (m, 5H), 5.14 (s, 2H), 4.32 (br, 2H), 2.77 (br, 2H), $2.33-2.13(\mathrm{~m}, 1 \mathrm{H}), 1.88(\mathrm{~d}, J=13.2 \mathrm{~Hz}, 2 \mathrm{H}), 1.55(\mathrm{qd}, J=12.6,4.5 \mathrm{~Hz}, 2 \mathrm{H})$.

${ }^{13}$ C NMR (125 MHz, $\left.\mathbf{C D C l}_{3}\right) \delta 155.15,136.65,128.66,128.25,128.10,119.45$ (qt, $J=286.8$, $36.9 \mathrm{~Hz}), 115.72(\mathrm{tq}, J=254.5,36.7 \mathrm{~Hz}), 67.47,43.13,38.66(\mathrm{t}, J=21.5 \mathrm{~Hz}), 23.99$.

${ }^{19}$ F NMR (282 MHz, CDCl 3$) \delta-81.90(\mathrm{~s}, 3 \mathrm{~F}),-121.99(\mathrm{dd}, J=14.3,3.1 \mathrm{~Hz}, 2 \mathrm{~F})$.

IR (film) $\mathbf{v}_{\max } 1701,1432,1305,1234,1189,1141,1021,842,731,696 \mathrm{~cm}^{-1}$.

HRMS (GC-EI-TOF) m/z calcd. for $\mathrm{C}_{15} \mathrm{H}_{16} \mathrm{~F}_{5} \mathrm{NO}_{2}\left(\left[\mathrm{M}^{*}\right]^{+}\right)$337.1096, found 337.1096. 
c) General procedures for heptafluoropropylation: To an oven-dried 40-mL vial equipped with a cross shaped stir bar were added AdNHSi(TMS) 3 (0.9 mmol, $358 \mathrm{mg}, 1.8$ equiv.), NaOAc (2.0 mmol, $164 \mathrm{mg}, 4.0$ equiv.), pyridine (1.0 mmol, $80 \mu \mathrm{L}, 2.0$ equiv.), substrate (0.5 mmol, 1.0 equiv.), $\mathrm{Cu}(\mathrm{MeCN})_{4} \mathrm{BF}_{4}$ (31.5 mg, $0.1 \mathrm{mmol}, 0.2$ equiv.), ligand (0.125 mmol, 0.25 equiv.) and photocatalyst $\operatorname{Ir}\left[(\text { ppy })_{2}(\right.$ bpy) $] \mathrm{PF}_{6}(2.0 \mathrm{mg}, 2.5 \mu \mathrm{mol}, 0.005$ equiv. $)$. Then, $\mathrm{MeCN}(2.5 \mathrm{~mL}, 0.2 \mathrm{M})$ was added via syringe, followed by addition of $\mathrm{TMSC}_{3} \mathrm{~F}_{7}(300 \mu \mathrm{L}, 1.5 \mathrm{mmol}, 3.0$ equiv.). After the reaction mixture was stirred for $10 \mathrm{~min}$, the vial septum was pierced by an $18 \mathrm{G}$ needle, which was left in the septum for the duration of the reaction to allow for air flow without solvent evaporation. The reaction vial was then placed in the Merck Integrated Photoreactor for $18 \mathrm{~h}$ under the following conditions: 45\% LED intensity, $500 \mathrm{rpm}$ stirring and $5200 \mathrm{rpm}$ fan speed. Work up and purification for each substrate are described below.

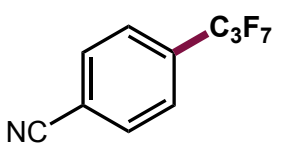

$\mathbf{5 8}, 52 \%$ yield

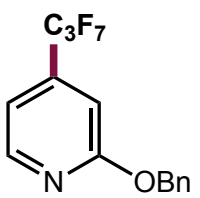

S2, 54\% yield<smiles>FC(F)(F)c1cnc2ccccc2n1</smiles>

S3, $48 \%$ yield

Figure S13. Heptafluoropropylated substrate scope 


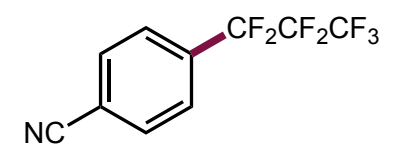

\section{4-(perfluoropropyl)benzonitrile (58)}

Prepared following the general procedure outlined above using $\operatorname{Ir}\left[(\mathrm{ppy})_{2}(\mathrm{bpy})\right] \mathrm{PF}_{6}(2.0 \mathrm{mg}, 2.5$ $\mu$ mol, 0.005 equiv.), $\mathrm{Cu}(\mathrm{MeCN})_{4} \mathrm{BF}_{4}$ (31.5 mg, $0.1 \mathrm{mmol}, 0.20$ equiv.), 4,4'-dimethyl-2,2'bipyridine (23 mg, $0.125 \mathrm{mmol}, 0.25$ equiv.), AdNHSi(TMS)3 (358 mg, $0.9 \mathrm{mmol}, 1.8$ equiv.), $\mathrm{NaOAc}$ (164 mg, $2.0 \mathrm{mmol}, 4.0$ equiv.), 4-bromobenzonitrile (91.0 mg, $0.5 \mathrm{mmol}, 1.0$ equiv.), Pyridine (1.0 mmol, $80 \mu \mathrm{l}, 2.0$ equiv. ) $\mathrm{TMSC}_{3} \mathrm{~F}_{7}$ reagent $(300 \mu \mathrm{L}, 1.5 \mathrm{mmol}, 3.0$ equiv.) and $\operatorname{MeCN}(2.5 \mathrm{~mL}, 0.2 \mathrm{M})$.

The final reaction mixture was diluted with EtOAc $(20 \mathrm{~mL})$ and then filtered over celite. After washing the celite with EtOAc three times, the organic solution was concentrated to yield the crude product as an oil. The product was purified via silica gel column chromatography (gradient 5 to $20 \%$ DCM in pentane) to yield the pure product ( $70 \mathrm{mg}, 52 \%$ yield).

${ }^{1}$ H NMR (500 MHz, $\left.\mathbf{C D C l}_{3}\right) \delta 7.83(\mathrm{dd}, J=8.0,0.9 \mathrm{~Hz}, 2 \mathrm{H}), 7.73(\mathrm{~d}, J=8.0 \mathrm{~Hz}, 2 \mathrm{H})$.

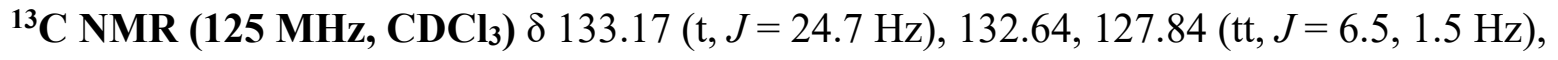
$118.18(\mathrm{qt}, J=288.5,34.1 \mathrm{~Hz}), 117.53,116.46(\mathrm{t}, J=1.9 \mathrm{~Hz}), 114.59(\mathrm{tt}, J=256.5,31.4 \mathrm{~Hz})$, $108.63(\mathrm{tq}, J=264.7,38.0 \mathrm{~Hz})$.

${ }^{19}$ F NMR (282 MHz, CDCl $)$ ) $\delta-79.93(\mathrm{t}, J=9.8 \mathrm{~Hz}, 3 \mathrm{~F}),-112.65(\mathrm{q}, J=9.8 \mathrm{~Hz}, 2 \mathrm{~F}),-126.21(\mathrm{~s}$, 2F).

Data are consistent with those reported in the literature: Huang, Y. J.; Ajitha, M. J.; Huang, K. W.; Zhang, Z. X; Weng, Z. Q. Dalton Trans. 2016, 45, 8468. 


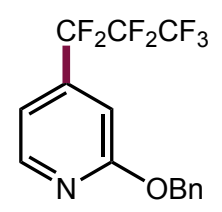

\section{2-(benzyloxy)-4-(perfluoropropyl)pyridine (S2)}

Prepared following the general procedure outlined above using $\operatorname{Ir}\left[(\mathrm{ppy})_{2}(\mathrm{bpy}) \mathrm{PF}_{6} \quad(2.0 \mathrm{mg}, 2.5\right.$ $\mu$ mol, 0.005 equiv.), $\mathrm{Cu}(\mathrm{MeCN})_{4} \mathrm{BF}_{4}$ (31.5 mg, $0.1 \mathrm{mmol}, 0.20$ equiv.), 4,4'-dimethyl-2,2'bipyridine (23 mg, $0.125 \mathrm{mmol}, 0.25$ equiv.), AdNHSi(TMS) 3 (358 mg, $0.9 \mathrm{mmol}, 1.8$ equiv.), $\mathrm{NaOAc}$ (164 mg, $2.0 \mathrm{mmol}, 4.0$ equiv.), 2-(benzyloxy)-4-bromopyridine (132 mg, $0.5 \mathrm{mmol}, 1.0$ equiv.), pyridine ( $1.0 \mathrm{mmol}, 80 \mu \mathrm{L}, 2.0$ equiv. ) $\mathrm{TMSC}_{3} \mathrm{~F}_{7}$ reagent ( $300 \mu \mathrm{L}, 1.5 \mathrm{mmol}, 3.0$ equiv.) and $\mathrm{MeCN}(2.5 \mathrm{~mL}, 0.2 \mathrm{M})$.

The final reaction mixture was diluted with EtOAc $(20 \mathrm{~mL})$ and then filtered over celite. After washing the celite with EtOAc three times, the organic solution was concentrated to yield the crude product as an oil. The product was purified via silica gel column chromatography (gradient 1 to $10 \%$ DCM in pentane) to yield the pure product ( $95 \mathrm{mg}, 54 \%$ yield).

${ }^{1}$ H NMR (400 MHz, CDCl $) \delta 8.35(\mathrm{~d}, J=5.4 \mathrm{~Hz}, 1 \mathrm{H}), 7.50-7.46(\mathrm{~m}, 2 \mathrm{H}), 7.43-7.32(\mathrm{~m}$, $3 \mathrm{H}), 7.07(\mathrm{~d}, J=5.4 \mathrm{~Hz}, 1 \mathrm{H}), 7.04(\mathrm{~s}, 1 \mathrm{H}), 5.44(\mathrm{~s}, 2 \mathrm{H})$.

${ }^{13}$ C NMR (125 MHz, CDCl $) \delta$ ) 164.04, 148.13, 139.77 (t, $\left.J=25.1 \mathrm{~Hz}\right), 136.70,128.70,128.27$, $128.24,117.93$ (qt, $J=287.5,33.9 \mathrm{~Hz}), 114.15(\mathrm{tt}, J=254.5,33.9 \mathrm{~Hz}), 113.86(\mathrm{t}, J=6.0 \mathrm{~Hz})$, $109.78(\mathrm{tt}, J=6.9,1.4 \mathrm{~Hz}), 108.63(\mathrm{tq}, J=265.1,37.8 \mathrm{~Hz}), 68.47$.

${ }^{19} \mathbf{F}$ NMR (376 MHz, CDCl $)$ ) $\delta-80.05(\mathrm{t}, J=9.8 \mathrm{~Hz}, 3 \mathrm{~F}),-113.77(\mathrm{q}, J=9.7 \mathrm{~Hz}, 2 \mathrm{~F}),-126.46$ (s, 2F).

IR (film) $v_{\max } 1610,1583,1471,1403,1375,1321,1245,1208,1175,1031,1089,1029,974$, $854,791,738,712,685 \mathrm{~cm}^{-1}$.

HRMS (ESI-TOF) $\mathrm{m} / \mathrm{z}$ calcd. for $\mathrm{C}_{15} \mathrm{H}_{11} \mathrm{~F}_{7} \mathrm{NO}\left([\mathrm{M}+\mathrm{H}]^{+}\right)$354.0723, found 354.0721. 


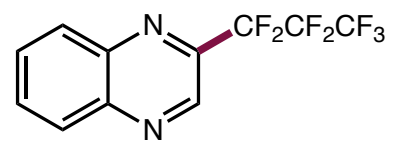

\section{2-(perfluoropropyl)quinoxaline (S3)}

Prepared following the general procedure outlined above using $\operatorname{Ir}\left[(\mathrm{ppy})_{2}(\mathrm{bpy})\right] \mathrm{PF}_{6}(2.0 \mathrm{mg}, 2.5$ umol, 0.005 equiv.), $\mathrm{Cu}(\mathrm{MeCN})_{4} \mathrm{BF}_{4}$ (31.5 mg, $0.1 \mathrm{mmol}, 0.20$ equiv.), 4,4'-dimethyl-2,2'bipyridine (23 mg, $0.125 \mathrm{mmol}, 0.25$ equiv.), AdNHSi(TMS)3 (358 mg, $0.9 \mathrm{mmol}, 1.8$ equiv.), $\mathrm{NaOAc}$ (164 mg, $2.0 \mathrm{mmol}, 4.0$ equiv.), 2-bromoquinoxaline (105 mg, $0.5 \mathrm{mmol}, 1.0$ equiv.), pyridine (1.0 mmol, $80 \mu \mathrm{l}, 2.0$ equiv. ), $\mathrm{TMSC}_{3} \mathrm{~F}_{7}$ reagent (300 $\mu \mathrm{L}, 1.5 \mathrm{mmol}, 3.0$ equiv.) and $\operatorname{MeCN}(2.5 \mathrm{~mL}, 0.2 \mathrm{M})$.

The final reaction mixture was diluted with EtOAc $(20 \mathrm{~mL})$ and then filtered over celite. After washing the celite with EtOAc three times, the organic solution was concentrated to yield the crude product as an oil. The product was purified via silica gel column chromatography (gradient 5 to $25 \%$ DCM in pentane) to yield the pure product ( $71 \mathrm{mg}, 48 \%$ yield).

${ }^{1}$ H NMR (400 MHz, CDCl $) \delta 9.15(\mathrm{~s}, 1 \mathrm{H}), 8.32-8.17(\mathrm{~m}, 2 \mathrm{H}), 8.01-7.85(\mathrm{~m}, 2 \mathrm{H})$.

${ }^{13}$ C NMR (125 MHz, CDCl $) \delta 143.65$ (d, $\left.J=1.5 \mathrm{~Hz}\right), 142.63(\mathrm{t}, J=25.7 \mathrm{~Hz}), 142.05$ (dq, $J=$

$4.5,2.2,1.6 \mathrm{~Hz}$ ), 141.31, 132.72, 131.70, 130.36, 129.66, 117.93 (qt, $J=287.7,33.8 \mathrm{~Hz}$ ), 112.85 (tt, $J=259.4,31.4 \mathrm{~Hz}), 108.99$ (tq, $J=266.9,36.6 \mathrm{~Hz})$.

${ }^{19}$ F NMR (376 MHz, CDCl $) \delta-80.04(\mathrm{t}, J=9.3 \mathrm{~Hz}, 3 \mathrm{~F}),-114.82(\mathrm{q}, J=9.2 \mathrm{~Hz}, 2 \mathrm{~F}),-126.02$ $(\mathrm{s}, 2 \mathrm{~F})$.

Data are consistent with those reported in the literature: Huang, Y. J.; Ajitha, M. J.; Huang, K. W.; Zhang, Z. X; Weng, Z. Q. Dalton Trans. 2016, 45, 8468. 


\section{d) Late-stage functionalization of drug molecules}<smiles>COc1cc(N([O+])S(=O)(=O)c2ccc(C(F)(F)F)cc2)nc(OC)n1</smiles>

\section{$N$-(2,6-dimethoxypyrimidin-4-yl)- $N$-methyl-4-(trifluoromethyl)benzenesulfonamide (59)}

Prepared following the general procedure outlined above using $\operatorname{Ir}\left[(\mathrm{F}-\mathrm{mppy})_{2}\right.$ (phen) $\mathrm{PF}_{6}(2.2 \mathrm{mg}$, $2.5 \mu \mathrm{mol}, 0.005$ equiv.), $\mathrm{CuBr}$ (14.3 mg, $0.1 \mathrm{mmol}, 0.20$ equiv.), 4,4'-dimethyl-2,2'-bipyridine (23 mg, 0.125 mmol, 0.25 equiv.), AdNHSi(TMS) 3 (358 mg, 0.9 mmol, 1.8 equiv.), NaOAc (164 mg, 2.0 mmol, 4.0 equiv.), 4-bromo- $N$-(2,6-dimethoxypyrimidin-4-yl)- $N$-methylbenzenesulfonamide (194 mg, 0.5 mmol, 1.0 equiv.), $\mathrm{TMSCF}_{3}$ reagent $(220 \mu \mathrm{L}, 1.5 \mathrm{mmol}, 3.0$ equiv.) and $\mathrm{MeCN}(3.0$ $\mathrm{ml}, 0.17 \mathrm{M})$. Integrated Photoreactor settings: $5200 \mathrm{rpm}$ fan speed, $500 \mathrm{rpm}$ stir rate, 40\% light intensity, 16 hours.

The final reaction mixture was diluted with EtOAc $(20 \mathrm{~mL})$ and then filtered over celite. After washing the celite with EtOAc three times, the organic solution was concentrated to yield the crude product as an oil. The product was purified via silica gel column chromatography (gradient 20 to $60 \%$ DCM in hexane) to yield the pure product as a white crystalline solid (150 mg, 80\% yield).

${ }^{1}$ H NMR (500 MHz, CDCl $) \delta 7.93(\mathrm{~d}, J=8.4 \mathrm{~Hz}, 2 \mathrm{H}), 7.75(\mathrm{~d}, J=8.4 \mathrm{~Hz}, 2 \mathrm{H}), 6.58(\mathrm{~s}, 1 \mathrm{H})$, $3.94(\mathrm{~s}, 3 \mathrm{H}), 3.83(\mathrm{~s}, 3 \mathrm{H}), 3.45(\mathrm{~s}, 3 \mathrm{H})$.

${ }^{13}$ C NMR (125 MHz, CDCl $) \delta 172.83,164.51,161.24,142.22$ (q, $\left.J=1.3 \mathrm{~Hz}\right), 135.07$ (q, $J=$ $33.2 \mathrm{~Hz}), 127.87,126.42$ (q, $J=3.7 \mathrm{~Hz}), 123.19$ (q, $J=273.1 \mathrm{~Hz}), 90.44,54.94,54.36,34.82$.

${ }^{19}$ F NMR (282 MHz, $\left.\mathbf{C D C l}_{3}\right) \delta-63.24(\mathrm{~s}, 3 \mathrm{~F})$.

IR (film) $v_{\max } 2954,1573,1461,1403,1321,1208,1165,1131,1089,1062,1029,974,836$, $791,738,712,685 \mathrm{~cm}^{-1}$.

HRMS (ESI-TOF) m/z calcd. for $\mathrm{C}_{14} \mathrm{H}_{15} \mathrm{~F}_{3} \mathrm{~N}_{3} \mathrm{O}_{4} \mathrm{~S}\left([\mathrm{M}+\mathrm{H}]^{+}\right)$378.0730, found 378.0730 . 


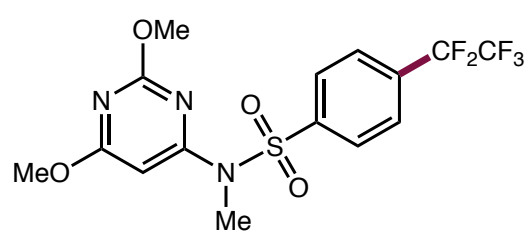

$N$-(2,6-dimethoxypyrimidin-4-yl)- $N$-methyl-4-(perfluoroethyl)benzenesulfonamide (65)

Prepared following the general procedure outlined above using $\operatorname{Ir}\left[(\mathrm{ppy})_{2}(\mathrm{bpy})\right] \mathrm{PF}_{6}(2.0 \mathrm{mg}, 2.5$ $\mu$ mol, 0.005 equiv.), $\mathrm{CuBr}$ (14.3 mg, $0.1 \mathrm{mmol}, 0.20$ equiv.), 4,4'-dimethyl-2,2'-bipyridine (23 mg, 0.125 mmol, 0.25 equiv.), AdNHSi(TMS) 3 (358 mg, 0.9 mmol, 1.8 equiv.), NaOAc (164 mg, 2.0 mmol, 4.0 equiv.), 4-bromo- $N$-(2,6-dimethoxypyrimidin-4-yl)- $N$-methylbenzenesulfonamide (194 $\mathrm{mg}, 0.5 \mathrm{mmol}, 1.0$ equiv.), $\mathrm{TMSC}_{2} \mathrm{~F}_{5}$ reagent $(260 \mu \mathrm{L}, 1.5 \mathrm{mmol}, 3.0$ equiv.) and $\mathrm{MeCN}(3.0 \mathrm{~mL}$, $0.17 \mathrm{M}$ ). Integrated Photoreactor settings: $5200 \mathrm{rpm}$ fan speed, $500 \mathrm{rpm}$ stir rate, 45\% light intensity, 18 hours.

The final reaction mixture was diluted with EtOAc $(20 \mathrm{~mL})$ and then filtered over celite. After washing the celite with EtOAc three times, the organic solution was concentrated to yield the crude product as an oil. The product was purified via silica gel column chromatography (gradient 15 to $50 \%$ DCM in pentane) to yield the pure product (162 mg, $76 \%$ yield).

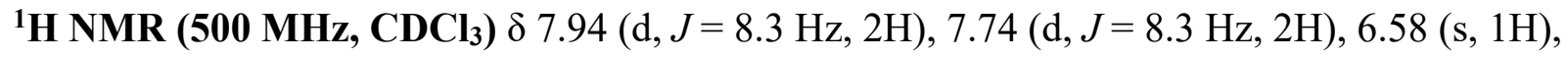
$3.95(\mathrm{~s}, 3 \mathrm{H}), 3.81(\mathrm{~s}, 3 \mathrm{H}), 3.46(\mathrm{~s}, 3 \mathrm{H})$.

${ }^{13}$ C NMR (125 MHz, CDCl $) \delta 172.85,164.51,161.27,142.57(\mathrm{~d}, J=1.7 \mathrm{~Hz}), 133.50(\mathrm{t}, J=$ $24.3 \mathrm{~Hz}), 127.72,127.64(\mathrm{~d}, J=6.5 \mathrm{~Hz}), 118.84$ (qt, $J=286.2,38.4 \mathrm{~Hz}), 112.78$ (tq, $J=254.5$ $38.7 \mathrm{~Hz}), 90.48,54.92,54.38,34.86$.

${ }^{19}$ F NMR (376 MHz, CDCl 3$) \delta-84.45(\mathrm{~s}, 3 \mathrm{~F}),-115.32(\mathrm{~s}, 2 \mathrm{~F})$.

IR (film) $\mathbf{v}_{\max } 2954,1573,1461,1404,1353,1285,1156,1096,1030,972,833,788,765,736$, $717,685 \mathrm{~cm}^{-1}$.

HRMS (ESI-TOF) m/z calcd. for $\mathrm{C}_{15} \mathrm{H}_{15} \mathrm{~F}_{5} \mathrm{~N}_{3} \mathrm{O}_{4} \mathrm{~S}\left([\mathrm{M}+\mathrm{H}]^{+}\right)$428.0698, found 428.0695 . 


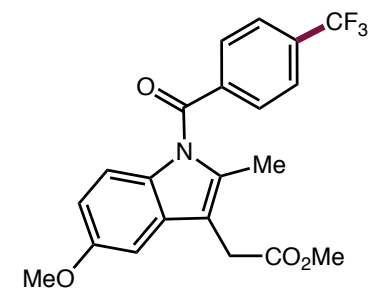

methyl 2-(5-methoxy-2-methyl-1-(4-(trifluoromethyl)benzoyl)-1H-indol-3-yl)acetate (60)

Prepared following the general procedure outlined above using $\operatorname{Ir}\left[(\mathrm{F}-\mathrm{mppy})_{2}(\mathrm{Phen})\right] \mathrm{PF}_{6}(2.2 \mathrm{mg}$, $2.5 \mu \mathrm{mol}, 0.005$ equiv.), $\mathrm{CuBr}$ (14.3 mg, $0.1 \mathrm{mmol}, 0.20$ equiv.), 4,4'-dimethyl-2,2'-bipyridine (23 mg, 0.125 mmol, 0.25 equiv.), AdNHSi(TMS) 3 (358 mg, 0.9 mmol, 1.8 equiv.), NaOAc (164 mg, 2.0 mmol, 4.0 equiv.), methyl 2-(1-(4-bromobenzoyl)-5-methoxy-2-methyl-1 $H$-indol-3-yl)acetate (210 mg, $0.5 \mathrm{mmol}, 1.0$ equiv.), $\mathrm{TMSCF}_{3}$ reagent $(220 \mu \mathrm{L}, 1.5 \mathrm{mmol}, 3.0$ equiv.) and $\mathrm{MeCN}(3.0$ ml, $0.17 \mathrm{M})$. Integrated Photoreactor settings: $5200 \mathrm{rpm}$ fan speed, $500 \mathrm{rpm}$ stir rate, 40\% light intensity, 16 hours.

The final reaction mixture was diluted with EtOAc $(20 \mathrm{~mL})$ and then filtered over celite. After washing the celite with EtOAc three times, the organic solution was concentrated to yield the crude product as an oil. The product was purified via silica gel column chromatography (gradient 1 to $5 \% \mathrm{Et}_{2} \mathrm{O}$ in DCM) to yield the pure solid product (158 $\mathrm{mg}, 78 \%$ yield).

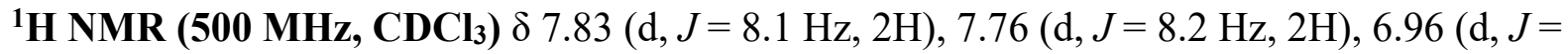
$2.6 \mathrm{~Hz}, 1 \mathrm{H}), 6.86(\mathrm{~d}, J=9.0 \mathrm{~Hz}, 1 \mathrm{H}), 6.68(\mathrm{dd}, J=9.1,2.5 \mathrm{~Hz}, 1 \mathrm{H}), 3.84(\mathrm{~s}, 3 \mathrm{H}), 3.71(\mathrm{~s}, 3 \mathrm{H})$, $3.67(\mathrm{~s}, 2 \mathrm{H}), 2.36(\mathrm{~s}, 3 \mathrm{H})$.

${ }^{13}$ C NMR (125 MHz, CDCl $) \delta 171.37,168.16,156.38,139.14(\mathrm{~d}, J=1.4 \mathrm{~Hz}), 135.98,134.28$ (q, $J=32.9 \mathrm{~Hz}), 130.97,130.79,130.02,125.96(\mathrm{q}, J=3.7 \mathrm{~Hz}), 123.64$ (q, $J=272.8 \mathrm{~Hz})$, $115.21,113.20,111.87,101.63,55.87,52.35,30.26,13.71$.

${ }^{19}$ F NMR (282 MHz, CDCl 3$) \delta-63.02(\mathrm{~s}, 3 \mathrm{~F})$.

IR (film) $\mathbf{v}_{\max } 2954,1737,1682,1608,1203,1108,1478,1437,1409,1358,1315,1214,1165$, $1129,1109,1065,1035,1017,973,926,837,767,735,690,666 \mathrm{~cm}^{-1}$.

HRMS (ESI-TOF) m/z calcd. for $\mathrm{C}_{21} \mathrm{H}_{19} \mathrm{~F}_{3} \mathrm{NO}_{4}\left([\mathrm{M}+\mathrm{H}]^{+}\right) 406.1261$, found 406.1260. 


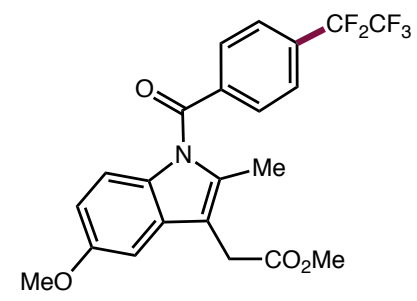

methyl 2-(5-methoxy-2-methyl-1-(4-(perfluoroethyl)benzoyl)-1H-indol-3-yl)acetate (66)

Prepared following the general procedure outlined above using $\operatorname{Ir}\left[(\mathrm{ppy})_{2}(\mathrm{bpy})\right] \mathrm{PF}_{6}(2.0 \mathrm{mg}, 2.5$ $\mu \mathrm{mol}, 0.005$ equiv.), $\mathrm{CuBr}$ (14.3 mg, $0.1 \mathrm{mmol}, 0.20$ equiv.), 4,4'-dimethyl-2,2'-bipyridine (23 mg, 0.125 mmol, 0.25 equiv.), AdNHSi(TMS) 3 (358 mg, 0.9 mmol, 1.8 equiv.), NaOAc (164 mg, 2.0 mmol, 4.0 equiv.), 4-bromo- $N$-(2,6-dimethoxypyrimidin-4-yl)- $N$-methylbenzenesulfonamide (194 mg, $0.5 \mathrm{mmol}, 1.0$ equiv.), $\mathrm{TMSC}_{2} \mathrm{~F}_{5}$ reagent ( $260 \mu \mathrm{L}, 1.5 \mathrm{mmol}, 3.0$ equiv.) and $\mathrm{MeCN}$ (3.0 ml, $0.17 \mathrm{M}$ ). Integrated Photoreactor settings: $5200 \mathrm{rpm}$ fan speed, $500 \mathrm{rpm}$ stir rate, $45 \%$ light intensity, 20 hours.

The final reaction mixture was diluted with EtOAc $(20 \mathrm{~mL})$ and then filtered over celite. After washing the celite with EtOAc three times, the organic solution was concentrated to yield the crude product as an oil. The product was purified via silica gel column chromatography (gradient 1 to $5 \% \mathrm{Et}_{2} \mathrm{O}$ in DCM) to yield the solid product (170 mg, $75 \%$ yield).

${ }^{1}$ H NMR (400 MHz, CDCl$) \delta 7.84(\mathrm{~d}, J=7.6 \mathrm{~Hz}, 2 \mathrm{H}), 7.74(\mathrm{~d}, J=8.2 \mathrm{~Hz}, 2 \mathrm{H}), 6.97(\mathrm{~d}, J=$ $2.5 \mathrm{~Hz}, 1 \mathrm{H}), 6.88(\mathrm{~d}, J=9.0 \mathrm{~Hz}, 1 \mathrm{H}), 6.68(\mathrm{dd}, J=9.0,2.6 \mathrm{~Hz}, 1 \mathrm{H}), 3.84(\mathrm{~s}, 3 \mathrm{H}), 3.71(\mathrm{~s}, 3 \mathrm{H})$, $3.67(\mathrm{~s}, 2 \mathrm{H}), 2.35(\mathrm{~s}, 3 \mathrm{H})$.

${ }^{13}$ C NMR (125 MHz, CDCl $) \delta 171.36,168.16,156.42,139.33$ (d, $\left.J=1.7 \mathrm{~Hz}\right), 135.91,132.63$ (t, $J=24.2 \mathrm{~Hz}), 130.99,130.79,129.89,127.21$ (t, $J=6.2 \mathrm{~Hz}), 119.02$ (qt, $J=285.9,38.7 \mathrm{~Hz})$, $115.22,113.27,113.12$ (tq, $J=254.5,38.5 \mathrm{~Hz}), 111.89,101.66,55.87,52.36,30.27,13.73$.

${ }^{19}$ F NMR (376 MHz, CDCl 3$) \delta-84.53(\mathrm{~s}, 3 \mathrm{~F}),-115.21(\mathrm{~s}, 2 \mathrm{~F})$.

IR (film) $\boldsymbol{v}_{\max } 2955,1739,1684,1610,1478,1410,1359,1323,1286,1207,1148,1096,1070$, $1037,974,927,841,762,707 \mathrm{~cm}^{-1}$.

HRMS (ESI-TOF) m/z calcd. for $\mathrm{C}_{22} \mathrm{H}_{19} \mathrm{~F}_{5} \mathrm{NO}_{4}\left([\mathrm{M}+\mathrm{H}]^{+}\right)$456.1228, found 456.1224. 


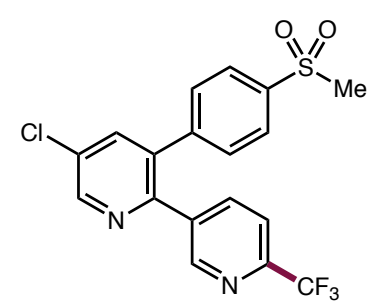

\section{5-chloro-3-(4-(methylsulfonyl)phenyl)-6'-(trifluoromethyl)-2,3'-bipyridine (61)}

Prepared following the general procedure outlined above using $\operatorname{Ir}\left[(\mathrm{F}-\mathrm{mppy})_{2}\right.$ (phen) $\mathrm{PF}_{6}(2.2 \mathrm{mg}$, $2.5 \mu \mathrm{mol}, 0.005$ equiv.), $\mathrm{CuBr}$ (14.3 mg, $0.1 \mathrm{mmol}, 0.20$ equiv.), 4,4'-dimethoxy-2,2'-bipyridine (27 mg, 0.125 mmol, 0.25 equiv.), AdNHSi(TMS) $)_{3}$ (358 mg, 0.9 mmol, 1.8 equiv.), NaOAc (164 mg, 2.0 mmol, 4.0 equiv.), 6'-bromo-5-chloro-3-(4-(methylsulfonyl)phenyl)-2,3'-bipyridine (212 mg, 0.5 mmol, 1.0 equiv.), $\mathrm{TMSCF}_{3}$ reagent $(220 \mu \mathrm{L}, 1.5 \mathrm{mmol}, 3.0$ equiv.) and $\mathrm{MeCN}$ (3.0 mL, $0.17 \mathrm{M}$ ). Integrated Photoreactor settings: $5200 \mathrm{rpm}$ fan speed, $500 \mathrm{rpm}$ stir rate, 40\% light intensity, 16 hours.

The final reaction mixture was diluted with $\mathrm{MeCN}(20 \mathrm{~mL})$ and then filtered over celite. After washing the celite with MeCN three times, the organic solution was concentrated to yield the crude product as an oil. The product was purified via silica gel column chromatography (gradient 1 to $6 \%$ EA in DCM) to yield the pure solid product (130 mg, 63\% yield).

${ }^{1}$ H NMR (500 MHz, CDCl$) \delta 8.75(\mathrm{~d}, J=2.3 \mathrm{~Hz}, 1 \mathrm{H}), 8.61(\mathrm{~d}, J=2.2 \mathrm{~Hz}, 1 \mathrm{H}), 7.93(\mathrm{~d}, J=$ $8.3 \mathrm{~Hz}, 2 \mathrm{H}), 7.89$ (dd, $J=8.2,2.2 \mathrm{~Hz}, 1 \mathrm{H}), 7.79$ (d, $J=2.3 \mathrm{~Hz}, 1 \mathrm{H}), 7.63(\mathrm{dd}, J=8.2,0.8 \mathrm{~Hz}$, 1H), $7.40(\mathrm{~d}, J=8.4 \mathrm{~Hz}, 2 \mathrm{H}), 3.09$ (s, 3H).

${ }^{13}$ C NMR (125 MHz, $\left.\mathbf{C D C l}_{3}\right) \delta 150.78,150.56,148.91,147.68$ (q, $\left.J=35.0 \mathrm{~Hz}\right), 143.10,140.85$, $138.61,138.42,137.08,135.89,132.31,130.47,128.34,121.40$ (q, $J=274.2 \mathrm{~Hz}), 120.19$ (q, $J=$ $2.7 \mathrm{~Hz}), 44.57$.

${ }^{19}$ F NMR (282 MHz, $\left.\mathrm{CDCl}_{3}\right) \delta-67.90(\mathrm{~s}, 3 \mathrm{~F})$.

IR (film) $v_{\max } 1599,1433,1374,1337,1314,1337,1175,1150,1090,1011,958,845,789,768$, $701 \mathrm{~cm}^{-1}$.

HRMS (ESI-TOF) m/z calcd. for $\mathrm{C}_{18} \mathrm{H}_{13} \mathrm{ClF}_{3} \mathrm{~N}_{2} \mathrm{O}_{2} \mathrm{~S}\left([\mathrm{M}+\mathrm{H}]^{+}\right)$413.0333, found 413.0331. 


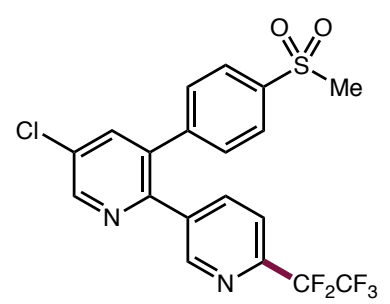

5-chloro-3-(4-(methylsulfonyl)phenyl)-6'-(perfluoroethyl)-2,3'-bipyridine (67)

Prepared following the general procedure outlined above using $\operatorname{Ir}\left[(\mathrm{ppy})_{2}(\mathrm{bpy})\right] \mathrm{PF}_{6}(2.0 \mathrm{mg}, 2.5$ $\mu$ mol, 0.005 equiv.), $\mathrm{CuBr}$ (14.3 mg, $0.1 \mathrm{mmol}, 0.20$ equiv.), 4,4'-dimethoxy-2,2'-bipyridine (27 mg, 0.125 mmol, 0.25 equiv.), AdNHSi(TMS) 3 (358 mg, 0.9 mmol, 1.8 equiv.), NaOAc (164 mg, $2.0 \mathrm{mmol}, 4.0$ equiv.), 6'-bromo-5-chloro-3-(4-(methylsulfonyl)phenyl)-2,3'-bipyridine (212 mg, $0.5 \mathrm{mmol}, 1.0$ equiv.), $\mathrm{TMSC}_{2} \mathrm{~F}_{5}$ reagent $(260 \mu \mathrm{L}, 1.5 \mathrm{mmol}, 3.0$ equiv.) and $\mathrm{MeCN}(3.0 \mathrm{~mL}, 0.17$ M). Integrated Photoreactor settings: $5200 \mathrm{rpm}$ fan speed, $500 \mathrm{rpm}$ stir rate, $45 \%$ light intensity, 18 hours.

The final reaction mixture was diluted with $\mathrm{MeCN}(20 \mathrm{~mL})$ and then filtered over celite. After washing the celite with MeCN three times, the organic solution was concentrated to yield the crude product as an oil. The product was purified via silica gel column chromatography (gradient 1 to $5 \%$ EA in DCM) to yield the solid product (157 mg, $68 \%$ yield).

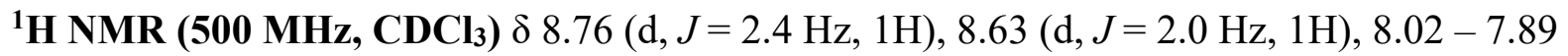
(m, 3H), $7.80(\mathrm{~d}, J=2.2 \mathrm{~Hz}, 1 \mathrm{H}), 7.67(\mathrm{dd}, J=8.2,0.8 \mathrm{~Hz}, 1 \mathrm{H}), 7.40(\mathrm{~d}, J=8.4 \mathrm{~Hz}, 2 \mathrm{H}), 3.09$ $(\mathrm{s}, 3 \mathrm{H})$.

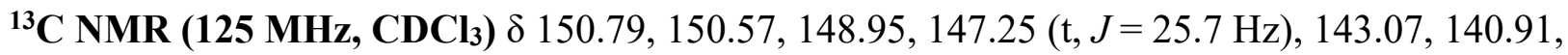
$138.49,138.44,137.03(\mathrm{~d}, J=1.4 \mathrm{~Hz}), 135.95,132.40,130.48,128.34,121.76$ (t, $J=4.4 \mathrm{~Hz})$, $118.88(\mathrm{qt}, J=286.7,37.5 \mathrm{~Hz}), 111.18(\mathrm{tq}, J=255.3,38.1 \mathrm{~Hz}), 44.59$.

${ }^{19}$ F NMR (282 MHz, $\left.\mathbf{C D C l}_{3}\right) \delta-83.18(\mathrm{~s}, 3 \mathrm{~F}),-117.40$ (s, 2F).

IR (film) $\boldsymbol{v}_{\max } 2958,1599,1431,1309,1203,1108,1089,1029,1011,980,957,906,841,768$, $732,707 \mathrm{~cm}^{-1}$.

HRMS (ESI-TOF) m/z calcd. for $\mathrm{C}_{19} \mathrm{H}_{13} \mathrm{ClF}_{5} \mathrm{~N}_{2} \mathrm{O}_{2} \mathrm{~S}\left([\mathrm{M}+\mathrm{H}]^{+}\right) 463.0301$, found 463.0313 . 


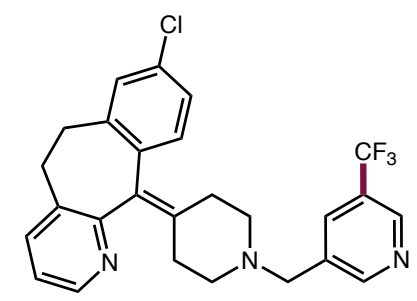

8-chloro-11-(1-((5-(trifluoromethyl)pyridin-3-yl)methyl)piperidin-4-ylidene)-6,11-dihydro$5 H$-benzo[5,6]cyclohepta[1,2-b]pyridine (62)

Prepared following the general procedure outlined above using $\operatorname{Ir}\left[(\mathrm{F}-\mathrm{mppy})_{2}\right.$ (phen) $\mathrm{PF}_{6}(2.2 \mathrm{mg}$, $2.5 \mu \mathrm{mol}, 0.005$ equiv.), $\mathrm{CuBr}$ (14.3 mg, $0.1 \mathrm{mmol}, 0.20$ equiv.), 4,4'-dimethyl-2,2'-bipyridine (23 mg, 0.125 mmol, 0.25 equiv.), AdNHSi(TMS) 3 (358 mg, 0.9 mmol, 1.8 equiv.), NaOAc (164 mg, $2.0 \mathrm{mmol}, 4.0$ equiv.), 11-(1-((5-bromopyridin-3-yl)methyl)piperidin-4-ylidene)-8-chloro-6,11dihydro-5H-benzo[5,6]cyclohepta[1,2-b]pyridine (240 mg, $0.5 \mathrm{mmol}, 1.0$ equiv.), TMSCF 3 reagent $(220 \mu \mathrm{L}, 1.5 \mathrm{mmol}, 3.0$ equiv.) and $\mathrm{MeCN}(3.0 \mathrm{~mL}, 0.17 \mathrm{M})$. Integrated Photoreactor settings: $5200 \mathrm{rpm}$ fan speed, $500 \mathrm{rpm}$ stir rate, 40\% light intensity, 16 hours.

The final reaction mixture was diluted with $\mathrm{MeCN}(20 \mathrm{~mL})$ and then filtered over celite. After washing the celite with MeCN three times, the organic solution was concentrated to yield the crude product as an oil. The product was purified via silica gel column chromatography (gradient 1 to $5 \% \mathrm{MeOH}$ in DCM) to yield the solid product (112 $\mathrm{mg}, 48 \%$ yield).

${ }^{1}$ H NMR (500 MHz, $\left.\mathbf{C D C l}_{3}\right) \delta 8.76(\mathrm{~s}, 1 \mathrm{H}), 8.72(\mathrm{~s}, 1 \mathrm{H}), 8.38(\mathrm{dd}, J=4.8,1.7 \mathrm{~Hz}, 1 \mathrm{H}), 7.92(\mathrm{~s}$, 1H), 7.42 (dd, $J=7.6,1.7 \mathrm{~Hz}, 1 \mathrm{H}), 7.14-7.08$ (m, 3H), 7.07 (dd, $J=7.6,4.8 \mathrm{~Hz}, 1 \mathrm{H}), 3.56$ (s, 2H), $3.45-3.30(\mathrm{~m}, 2 \mathrm{H}), 2.88-2.74(\mathrm{~m}, 2 \mathrm{H}), 2.74-2.67(\mathrm{~m}, 2 \mathrm{H}), 2.53(\mathrm{ddd}, J=14.1,9.8,4.3$ Hz, 1H), 2.44 (ddd, $J=13.8,9.7,4.3 \mathrm{~Hz}, 1 \mathrm{H}), 2.38-2.29$ (m, 2H), 2.20 (qd, $J=10.8,3.4 \mathrm{~Hz}$, 2H).

${ }^{13}$ C NMR (125 MHz, CDCl 3$) ~ \delta 157.48,153.51$ (q, $\left.J=1.5 \mathrm{~Hz}\right), 146.70,145.46$ (q, $\left.J=4.1 \mathrm{~Hz}\right)$, 139.60, 138.37, 137.83, 137.48, 134.59, 133.55 (q, $J=3.6 \mathrm{~Hz}), 133.51,133.10,132.84,130.87$, 129.06, 126.60 (q, $J=32.6 \mathrm{~Hz}), 126.15,123.64$ (q, $J=272.5 \mathrm{~Hz}), 122.28,59.57,54.90,54.84$, 31.90, 31.56, 30.95, 30.72. ${ }^{19}$ F NMR (282 MHz, CDCl $) \delta-62.35$ (s, 3F).

Data are consistent with those reported in the literature: Le, C.; Chen, T. Q.; Liang, T.; Zhang, P.; MacMillan, D. W. C. Science 2018, 360, 1010. 


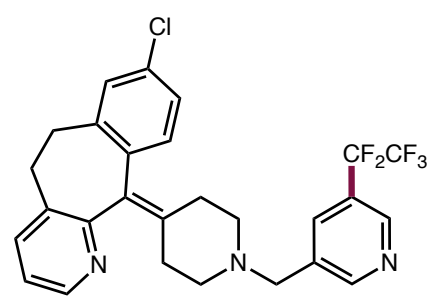

8-chloro-11-(1-((5-(perfluoroethyl)pyridin-3-yl)methyl)piperidin-4-ylidene)-6,11-dihydro$5 H$-benzo[5,6]cyclohepta[1,2-b]pyridine (68)

Prepared following the general procedure outlined above using $\operatorname{Ir}\left[(\mathrm{ppy})_{2}(\mathrm{bpy})\right] \mathrm{PF}_{6}(2.0 \mathrm{mg}, 2.5$ $\mu$ mol, 0.005 equiv.), $\mathrm{CuBr}$ (14.3 mg, $0.1 \mathrm{mmol}, 0.20$ equiv.), 4,4'-dimethyl-2,2'-bipyridine (23 mg, 0.125 mmol, 0.25 equiv.), AdNHSi(TMS) 3 (358 mg, 0.9 mmol, 1.8 equiv.), NaOAc (164 mg, 2.0 mmol, 4.0 equiv.), 11-(1-((5-bromopyridin-3-yl)methyl)piperidin-4-ylidene)-8-chloro-6,11dihydro-5H-benzo[5,6]cyclohepta[1,2-b]pyridine (240 mg, $0.5 \mathrm{mmol}, 1.0$ equiv.), $\mathrm{TMSC}_{2} \mathrm{~F}_{5}$ reagent $(260 \mu \mathrm{L}, 1.5 \mathrm{mmol}, 3.0$ equiv.) and $\mathrm{MeCN}(2.5 \mathrm{~mL}, 0.2 \mathrm{M})$. Integrated Photoreactor settings: $5200 \mathrm{rpm}$ fan speed, $500 \mathrm{rpm}$ stir rate, 45\% light intensity, 18 hours.

The final reaction mixture was diluted with $\mathrm{MeCN}(20 \mathrm{~mL})$ and then filtered over celite. After washing the celite with MeCN three times, the organic solution was concentrated to yield the crude

product as an oil. The product was purified via silica gel column chromatography (gradient 1 to $5 \% \mathrm{MeOH}$ in DCM) to yield the solid product (143 $\mathrm{mg}, 55 \%$ yield).

${ }^{1}$ H NMR (500 MHz, CDCl$) \delta 8.73(\mathrm{~s}, 1 \mathrm{H}), 8.71(\mathrm{~s}, 1 \mathrm{H}), 8.37(\mathrm{dd}, J=4.8,1.7 \mathrm{~Hz}, 1 \mathrm{H}), 7.89(\mathrm{~d}$, $J=2.2 \mathrm{~Hz}, 1 \mathrm{H}), 7.41(\mathrm{dd}, J=7.7,1.7 \mathrm{~Hz}, 1 \mathrm{H}), 7.14-7.09(\mathrm{~m}, 3 \mathrm{H}), 7.06(\mathrm{dd}, J=7.7,4.8 \mathrm{~Hz}$, 1H), $3.56(\mathrm{~s}, 2 \mathrm{H}), 3.43-3.29(\mathrm{~m}, 2 \mathrm{H}), 2.86-2.74(\mathrm{~m}, 2 \mathrm{H}), 2.72-2.66(\mathrm{~m}, 2 \mathrm{H}), 2.52(\mathrm{ddd}, J=$ 14.1, 9.8, 4.3 Hz, 1H), $2.43(\mathrm{ddd}, J=13.8,9.7,4.3 \mathrm{~Hz}, 1 \mathrm{H}), 2.38-2.28(\mathrm{~m}, 2 \mathrm{H}), 2.23-2.15(\mathrm{~m}$, $2 \mathrm{H})$.

${ }^{13}$ C NMR (125 MHz, CDCl $) \delta 157.43,153.59$ (t, $\left.J=1.8 \mathrm{~Hz}\right), 146.64,146.33(\mathrm{t}, J=6.6 \mathrm{~Hz})$, 139.57, 138.30, 137.79, 137.43, 134.64 (t, $J=6.3 \mathrm{~Hz}), 134.58,133.47,133.06,132.78,130.81$, $129.01,126.09,124.74(\mathrm{t}, J=24.2 \mathrm{~Hz}), 122.23,118.87$ (qt, $J=285.9,38.7 \mathrm{~Hz}), 112.86$ (tq, $J=$ 252.5, 38.8 Hz), 59.45, 54.81, 54.73, 31.84, 31.50, 30.90, 30.67.

${ }^{19}$ F NMR (282 MHz, $\left.\mathbf{C D C l}_{3}\right) \delta-84.86(\mathrm{~s}, 3 \mathrm{~F}),-115.41$ (s, 2F).

IR (film) $\mathbf{v}_{\max } 2920,1585,1478,1437,1365,1338,1299,1196,1125,1097,1013,858,828$, $800,734,715 \mathrm{~cm}^{-1}$.

HRMS (ESI-TOF) m/z calcd. for $\mathrm{C}_{27} \mathrm{H}_{24} \mathrm{~F}_{5} \mathrm{~N}_{3} \mathrm{Cl}\left([\mathrm{M}+\mathrm{H}]^{+}\right)$520.1573, found 520.1573. 


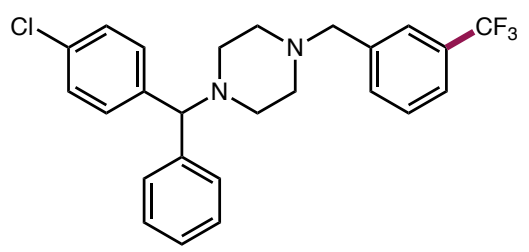

1-((4-chlorophenyl)(phenyl)methyl)-4-(3-(trifluoromethyl)benzyl)piperazine (63)

Prepared following the general procedure outlined above using $\operatorname{Ir}\left[(\mathrm{F}-\mathrm{mppy})_{2}(\mathrm{phen})\right] \mathrm{PF}_{6}(2.2 \mathrm{mg}$, $2.5 \mu \mathrm{mol}, 0.005$ equiv.), $\mathrm{CuBr}$ (14.3 mg, $0.1 \mathrm{mmol}, 0.20$ equiv.), 4,4'-dimethoxy-2,2'-bipyridine (27 mg, 0.125 mmol, 0.25 equiv.), AdNHSi(TMS) $)_{3}$ (358 mg, 0.9 mmol, 1.8 equiv.), NaOAc (164 mg, 2.0 mmol, 4.0 equiv.), 1-(3-bromobenzyl)-4-((4-chlorophenyl)(phenyl)methyl)-piperazine (230 mg, $0.5 \mathrm{mmol}, 1.0$ equiv.), $\mathrm{TMSCF}_{3}$ reagent $(220 \mu \mathrm{L}, 1.5 \mathrm{mmol}, 3.0$ equiv.) and $\mathrm{MeCN}(3.0$ mL, $0.17 \mathrm{M}$ ). Integrated Photoreactor settings: $5200 \mathrm{rpm}$ fan speed, $500 \mathrm{rpm}$ stir rate, 40\% light intensity, 16 hours.

The final reaction mixture was diluted with $\mathrm{MeCN}(20 \mathrm{~mL})$ and then filtered over celite. After washing the celite with MeCN three times, the organic solution was concentrated to yield the crude product as an oil. The product was purified via silica gel column chromatography (gradient 1 to $6 \%$ acetone in DCM) to yield the pure solid product (144 $\mathrm{mg}, 65 \%$ yield).

${ }^{1}$ H NMR (500 MHz, $\left.\mathbf{C D C l}_{3}\right) \delta 7.57(\mathrm{~s}, 1 \mathrm{H}), 7.49(\mathrm{~d}, J=8.4 \mathrm{~Hz}, 2 \mathrm{H}), 7.41(\mathrm{~d}, J=7.7 \mathrm{~Hz}, 1 \mathrm{H})$, $7.38-7.33(\mathrm{~m}, 4 \mathrm{H}), 7.28-7.23(\mathrm{~m}, 4 \mathrm{H}), 7.21-7.16(\mathrm{~m}, 1 \mathrm{H}), 4.22(\mathrm{~s}, 1 \mathrm{H}), 3.56(\mathrm{~s}, 2 \mathrm{H}), 2.50-$ $2.38(\mathrm{~m}, 8 \mathrm{H})$.

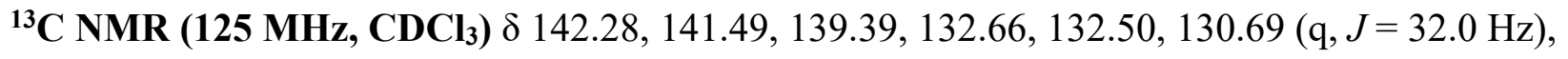
129.33, 128.77, 128.71, 128.38, 127.98, 127.28, 125.81 (q, $J=3.8 \mathrm{~Hz}), 124.36$ (q, $J=273.4 \mathrm{~Hz})$, $124.03(\mathrm{q}, J=3.9 \mathrm{~Hz}), 75.58,62.55,53.44,51.90$.

${ }^{19}$ F NMR (470 MHz, $\left.\mathrm{CDCl}_{3}\right) \delta-62.65$ (s, 3F).

IR (film) $\boldsymbol{v}_{\max } 2811,1669,1487,1452,1326,1162,1089,1071,1007,852,803,791,757,719$, $700,663 \mathrm{~cm}^{-1}$.

HRMS (ESI-TOF) m/z calcd. for $\mathrm{C}_{25} \mathrm{H}_{25} \mathrm{~F}_{3} \mathrm{~N}_{2} \mathrm{Cl}\left([\mathrm{M}+\mathrm{H}]^{+}\right) 445.1653$, found 445.1651 . 


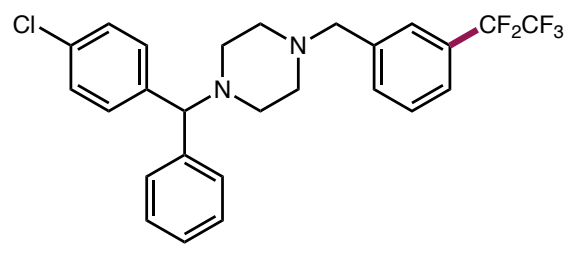

1-((4-chlorophenyl)(phenyl)methyl)-4-(3-(perfluoroethyl)benzyl)piperazine (69)

Prepared following the general procedure outlined above using $\operatorname{Ir}\left[(\mathrm{ppy})_{2}(\mathrm{bpy})\right] \mathrm{PF}_{6}(2.0 \mathrm{mg}, 2.5$ $\mu \mathrm{mol}, 0.005$ equiv.), $\mathrm{CuBr}$ (14.3 mg, $0.1 \mathrm{mmol}, 0.20$ equiv.), 4,4'- dimethoxy-2,2'-bipyridine (27 mg, 0.125 mmol, 0.25 equiv.), AdNHSi(TMS) 3 (358 mg, 0.9 mmol, 1.8 equiv.), NaOAc (164 mg, $2.0 \mathrm{mmol}, 4.0$ equiv.), 1-(3-bromobenzyl)-4-((4-chlorophenyl)(phenyl)methyl)-piperazine (230 mg, 0.5 mmol, 1.0 equiv.), $\mathrm{TMSC}_{2} \mathrm{~F}_{5}$ reagent $(260 \mu \mathrm{L}, 1.5 \mathrm{mmol}, 3.0$ equiv.) and $\mathrm{MeCN}$ (3.0 mL, $0.17 \mathrm{M}$ ). Integrated Photoreactor settings: $5200 \mathrm{rpm}$ fan speed, $500 \mathrm{rpm}$ stir rate, 45\% light intensity, 16 hours.

The final reaction mixture was diluted with $\mathrm{MeCN}(20 \mathrm{~mL})$ and then filtered over celite. After washing the celite with MeCN three times, the organic solution was concentrated to yield the crude product as an oil. The product was purified via silica gel column chromatography (gradient 1 to $5 \%$ acetone in DCM) to yield the solid product (168 $\mathrm{mg}, 68 \%$ yield).

${ }^{1}$ H NMR (500 MHz, $\left.\mathbf{C D C l}_{3}\right) \delta 7.54(\mathrm{~s}, 1 \mathrm{H}), 7.51(\mathrm{~d}, J=7.6 \mathrm{~Hz}, 1 \mathrm{H}), 7.49-7.44(\mathrm{~m}, 1 \mathrm{H}), 7.43$ $(\mathrm{d}, J=7.6 \mathrm{~Hz}, 1 \mathrm{H}), 7.37-7.33(\mathrm{~m}, 4 \mathrm{H}), 7.28-7.22(\mathrm{~m}, 4 \mathrm{H}), 7.20-7.16(\mathrm{~m}, 1 \mathrm{H}), 4.21(\mathrm{~s}, 1 \mathrm{H})$, $3.56(\mathrm{~s}, 2 \mathrm{H}), 2.46(\mathrm{br}, 8 \mathrm{H})$.

${ }^{13}$ C NMR (125 MHz, CDCl $) \delta 142.31,141.51,139.37,132.67,132.65,129.90,129.31,128.77$, $128.76(\mathrm{t}, J=22.5 \mathrm{~Hz}), 128.71,127.96,127.28,126.97$ (t, $J=6.3 \mathrm{~Hz}), 125.25$ (t, $J=6.3 \mathrm{~Hz})$, $119.23(\mathrm{dt}, J=286.0,39.5 \mathrm{~Hz}), 113.56(\mathrm{tq}, J=253.5,38.0 \mathrm{~Hz}), 75.60,62.49,53.38,51.89$.

${ }^{19}$ F NMR (470 MHz, $\left.\mathbf{C D C l}_{3}\right) \delta-82.69(\mathrm{~s}, 3 \mathrm{~F}),-116.36(\mathrm{~s}, 2 \mathrm{~F})$.

IR (film) $\boldsymbol{v}_{\max } 2811,1663,1488,1452,1337,1292,1089,1205,1136,1090,1008,856,803$, $757,719,704 \mathrm{~cm}^{-1}$.

HRMS (ESI-TOF) m/z calcd. for $\mathrm{C}_{26} \mathrm{H}_{25} \mathrm{~F}_{5} \mathrm{~N}_{2} \mathrm{Cl}\left([\mathrm{M}+\mathrm{H}]^{+}\right) 495.1621$, found 495.1620 . 


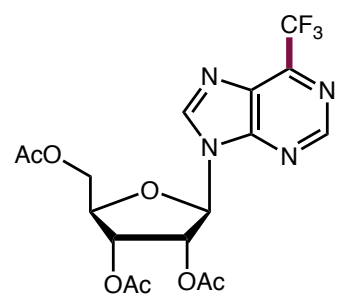

(2R,3R,4R,5R)-2-(acetoxymethyl)-5-(6-(trifluoromethyl)-9H-purin-9-yl)tetrahydrofuran-

\section{3,4-diyl diacetate (64)}

Prepared following the general procedure outlined above using $\operatorname{Ir}\left[(\mathrm{F}-\mathrm{mppy})_{2}\right.$ (phen) $\mathrm{PF}_{6}(2.2 \mathrm{mg}$, $2.5 \mu \mathrm{mol}, 0.005$ equiv.), $\mathrm{CuBr}$ (14.3 mg, $0.1 \mathrm{mmol}, 0.20$ equiv.), 4,4'-dimethoxy-2,2'-bipyridine (27 mg, 0.125 mmol, 0.25 equiv.), AdNHSi(TMS) 3 (358 mg, 0.9 mmol, 1.8 equiv.), NaOAc (164 mg, 2.0 mmol, 4.0 equiv.), (2R,3R,4R,5R)-2-(acetoxymethyl)-5-(6-bromo-9H-purin-9yl)tetrahydrofuran-3,4-diyl diacetate (230 mg, $0.5 \mathrm{mmol}, 1.0$ equiv.), $\mathrm{TMSCF}_{3}$ reagent $(220 \mu \mathrm{L}$, $1.5 \mathrm{mmol}, 3.0$ equiv.) and $\mathrm{MeCN}(3.0 \mathrm{~mL}, 0.17 \mathrm{M})$. Integrated Photoreactor settings: $5200 \mathrm{rpm}$ fan speed, $500 \mathrm{rpm}$ stir rate, $40 \%$ light intensity, 15 hours.

The final reaction mixture was diluted with $\mathrm{MeCN}(20 \mathrm{~mL})$ and then filtered over celite. After washing the celite with MeCN three times, the organic solution was concentrated to yield the crude product as an oil. The product was purified via silica gel column chromatography (gradient 1 to $10 \%$ acetone in DCM) to yield the pure solid product (116 mg, 52\% yield).

${ }^{1}$ H NMR (500 MHz, CDCl $) \delta 9.09$ (s, 1H), $8.43(\mathrm{~s}, 1 \mathrm{H}), 6.28(\mathrm{~d}, J=5.2 \mathrm{~Hz}, 1 \mathrm{H}), 5.95$ (t, $J=$ $5.4 \mathrm{~Hz}, 1 \mathrm{H}), 5.62$ (t, $J=5.2 \mathrm{~Hz}, 1 \mathrm{H}), 4.48$ (q, $J=4.2 \mathrm{~Hz}, 1 \mathrm{H}), 4.40$ (qd, $J=12.4,3.9 \mathrm{~Hz}, 2 \mathrm{H})$, $2.14(\mathrm{~s}, 3 \mathrm{H}), 2.10(\mathrm{~s}, 3 \mathrm{H}), 2.07(\mathrm{~s}, 3 \mathrm{H})$.

${ }^{13}$ C NMR (125 MHz, CDCl $) \delta 170.31,169.66,169.46,153.33,152.33,145.83,145.81$ (q, $J=$ $36.2 \mathrm{~Hz}), 130.89,120.61$ (q, $J=275.0 \mathrm{~Hz}), 86.89,80.70,73.16,70.55,62.96,20.81,20.58$, 20.42 .

${ }^{19}$ F NMR (470 MHz, $\left.\mathrm{CDCl}_{3}\right) \delta-66.25(\mathrm{~s}, 3 \mathrm{~F})$.

Data are consistent with those reported in the literature: Veliz, E. A.; Stephens, O. M.; Beal, P. A. Org. Lett. 2001, 3, 2969. 


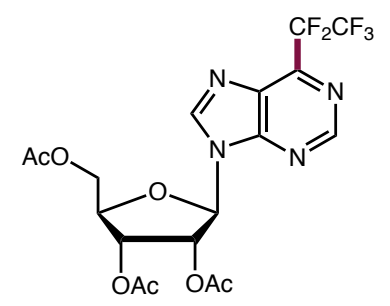

(2R,3R,4R,5R)-2-(acetoxymethyl)-5-(6-(perfluoroethyl)-9H-purin-9-yl)tetrahydrofuran-3,4diyl diacetate (70)

Prepared following the general procedure outlined above using $\operatorname{Ir}\left[(\mathrm{ppy})_{2}(\mathrm{bpy})\right] \mathrm{PF}_{6}(2.0 \mathrm{mg}, 2.5$ $\mu$ mol, 0.005 equiv.), $\mathrm{CuBr}$ (14.3 mg, $0.1 \mathrm{mmol}, 0.20$ equiv.), 4,4'-dimethoxy-2,2'-bipyridine (27 mg, 0.125 mmol, 0.25 equiv.), AdNHSi(TMS) 3 (358 mg, 0.9 mmol, 1.8 equiv.), NaOAc (164 mg, 2.0 mmol, 4.0 equiv.), (2R,3R,4R,5R)-2-(acetoxymethyl)-5-(6-bromo-9H-purin-9-yl)tetrahydrofuran-3,4-diyl diacetate ( $230 \mathrm{mg}, 0.5 \mathrm{mmol}, 1.0$ equiv.), $\mathrm{TMSC}_{2} \mathrm{~F}_{5}$ reagent $(260 \mu \mathrm{L}, 1.5$ mmol, 3.0 equiv.) and $\mathrm{MeCN}(3.0 \mathrm{~mL}, 0.17 \mathrm{M})$. Integrated Photoreactor settings: $5200 \mathrm{rpm}$ fan speed, $500 \mathrm{rpm}$ stir rate, $45 \%$ light intensity, 18 hours.

The final reaction mixture was diluted with $\mathrm{MeCN}(20 \mathrm{~mL})$ and then filtered over celite. After washing the celite with MeCN three times, the organic solution was concentrated to yield the crude product as an oil. The product was purified via silica gel column chromatography (gradient 1 to $8 \%$ acetone in DCM) to yield the solid product (119 $\mathrm{mg}, 48 \%$ yield).

${ }^{1}$ H NMR (500 MHz, CDCl $) \delta 9.13(\mathrm{~s}, 1 \mathrm{H}), 8.43(\mathrm{~s}, 1 \mathrm{H}), 6.29(\mathrm{~d}, J=5.1 \mathrm{~Hz}, 1 \mathrm{H}), 5.96(\mathrm{t}, J=$ $5.3 \mathrm{~Hz}, 1 \mathrm{H}), 5.64$ (t, $J=5.2 \mathrm{~Hz}, 1 \mathrm{H}), 4.49$ (td, $J=4.5,3.1 \mathrm{~Hz}, 1 \mathrm{H}), 4.41$ (qd, $J=14.7,3.8 \mathrm{~Hz}$, 2H), $2.15(\mathrm{~s}, 3 \mathrm{H}), 2.11(\mathrm{~s}, 3 \mathrm{H}), 2.09(\mathrm{~s}, 3 \mathrm{H})$.

${ }^{13}$ C NMR (125 MHz, $\left.\mathbf{C D C l}_{3}\right) \delta 170.33,169.68,169.48,153.17,152.26,145.80(\mathrm{t}, J=27.5 \mathrm{~Hz}$, $1 \mathrm{H}), 145.79,132.49,118.84(\mathrm{qt}, J=287.2,36.1 \mathrm{~Hz}), 111.10(\mathrm{tq}, J=257.7,39.2 \mathrm{~Hz}), 86.94$, $80.72,73.21,70.55,62.98,20.84,20.60,20.47$.

${ }^{19}$ F NMR (470 MHz, CDCl$\left._{3}\right) \delta-82.69$ (s, 3F), -116.36 (s, 2F).

IR (film) $v_{\max } 1745,1595,1498,1373,1328,1204,1125,1032,907,853,808,746 \mathrm{~cm}^{-1}$.

HRMS (ESI-TOF) m/z calcd. for $\mathrm{C}_{18} \mathrm{H}_{18} \mathrm{~F}_{5} \mathrm{~N}_{4} \mathrm{O}_{7}\left([\mathrm{M}+\mathrm{H}]^{+}\right)$497.1090, found 497.1092. 


\section{e) Additional examples \& limitations}

Figure S14. Additional examples \& limitations

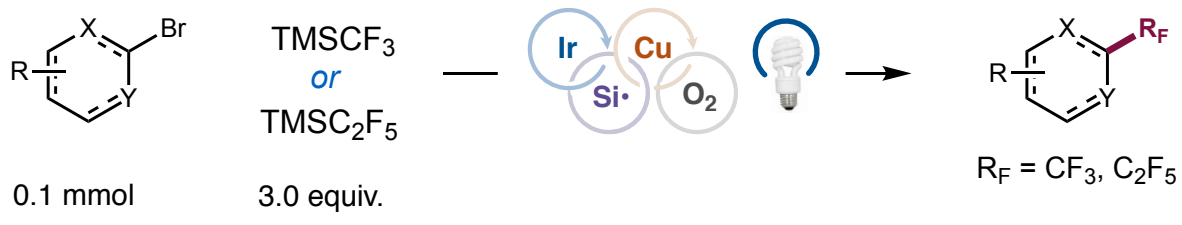<smiles>Nc1cnccc1C(F)(F)F</smiles>

$0 \%$ yield $^{a}$<smiles>FC(F)(F)C(F)(F)C(F)(F)C(F)(F)C(F)(F)F</smiles>

$10 \%$ yield $^{b}$

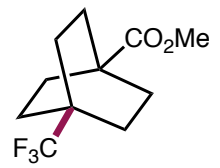

$30 \%$ yield $^{a}$<smiles>CC(C)(C)OC(=O)N1CCn2c(nnc2C(F)(F)F)C1</smiles>

$10 \%$ yield $^{a}$<smiles>Cn1cnc(C(F)(F)F)n1</smiles>

$23 \%$ yield $^{b}$

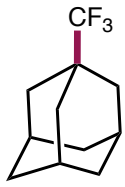

$20 \%$ yield $^{a}$<smiles>Cc1cc(C#N)cc([N+](=O)[O-])c1C(F)(F)F</smiles>

$40 \%$ yield $^{a}$

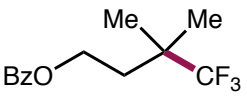

$0 \%$ yield $^{a}$<smiles>COC(=O)c1nc(C(F)(F)C(F)(F)C(F)(F)C(F)(F)C(F)(F)F)cn1C</smiles>

$12 \%$ yield $^{b}$<smiles>CC1(C(F)(F)F)CCN(C(=O)c2ccccc2)CC1</smiles>

$0 \%$ yield $^{a}$

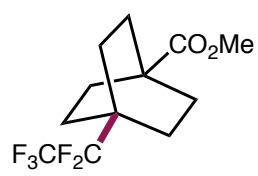

$0 \%$ yield $^{b}$

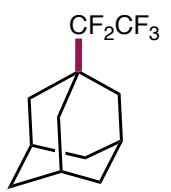

$0 \%$ yield $^{b}$

General reaction conditions: ${ }^{a}$ Performed with photocatalyst $\operatorname{Ir}\left[(\mathrm{F}-\mathrm{mppy})_{2}(\mathrm{phen})\right] \mathrm{PF}_{6}(1 \mathrm{~mol} \%), \mathrm{CuBr}(20 \mathrm{~mol} \%)$, 4,4'-dimethyl-2,2'-bipyridine ( $25 \mathrm{~mol} \%$ ), $\mathrm{TMS}_{3} \mathrm{SiAdNH}$ reagent (1.8 equiv), NaOAc (4 equiv), aryl or alkyl bromide $(0.1 \mathrm{mmol})$, and $\mathrm{TMSCF}_{3}$ (3 equiv) in $\mathrm{MeCN}(0.1 \mathrm{M}) .{ }^{b}$ Performed with photocatalyst $\operatorname{Ir}\left[(\mathrm{ppy})_{2}(\mathrm{bpy})\right] \mathrm{PF}_{6}$ (1 mol\%), CuBr (20 mol\%), 4,4'-dimethyl-2,2'-bipyridine (25 mol\%), TMS 3 SiAdNH reagent (1.8 equiv), $\mathrm{NaOAc}$ ( 4 equiv), aryl or alkyl bromide $(0.1 \mathrm{mmol})$, and $\mathrm{TMSC}_{2} \mathrm{~F}_{5}$ (3 equiv) in $\mathrm{MeCN}(0.1 \mathrm{M})$. Yields determined by ${ }^{19} \mathrm{~F}$ NMR analysis using 1,4-difluorobenzene as internal standard.

Note: In terms of aryl bromide limitations, we have observed diminished yields with certain electron-rich and/or nitrogen-rich heteroarenes. O,o-disubstitution is tolerated, albeit in moderate yields. In terms of alkyl bromide limitations, tertiary substrates are generally not amenable to the transformation; however, useful levels of trifluoromethylation were observed on adamantane and [2,2,2]-bicyclooctane scaffolds. 


\section{5) NMR Spectra}

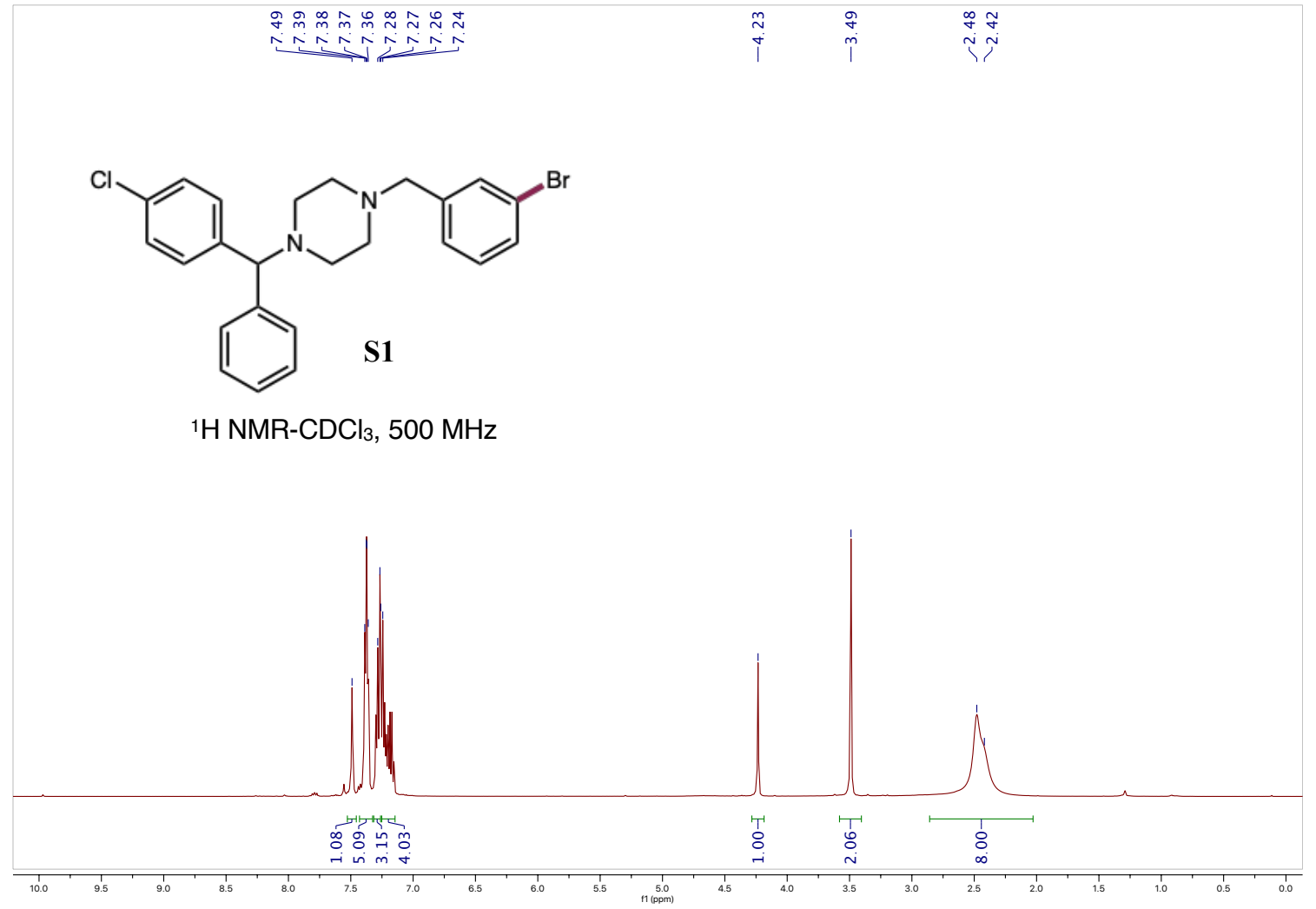

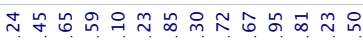

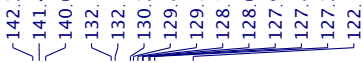

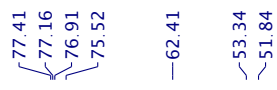

$\mathrm{Cl}$<smiles>CC(=O)NOC(C)=O</smiles>

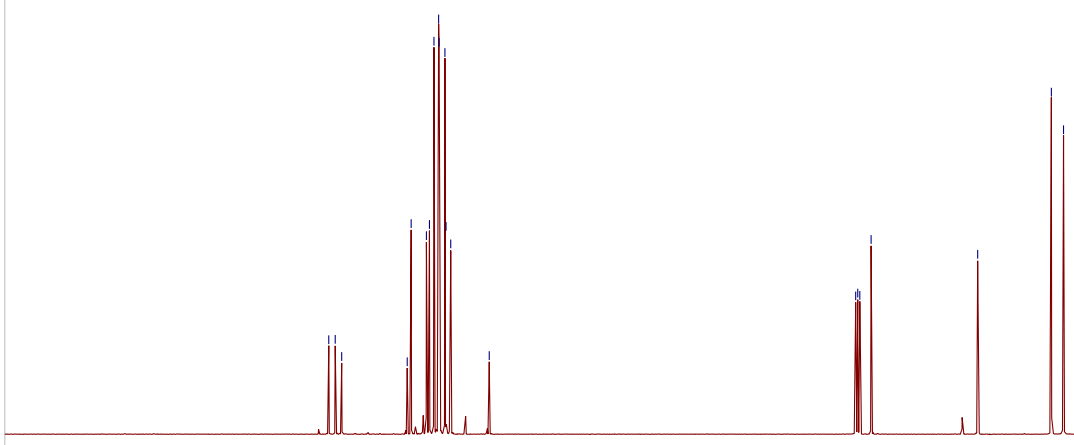

${ }_{180}^{1}$
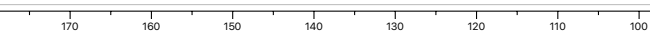

$\underset{\substack{1 \\ 900}}{\operatorname{lppm})}$ 

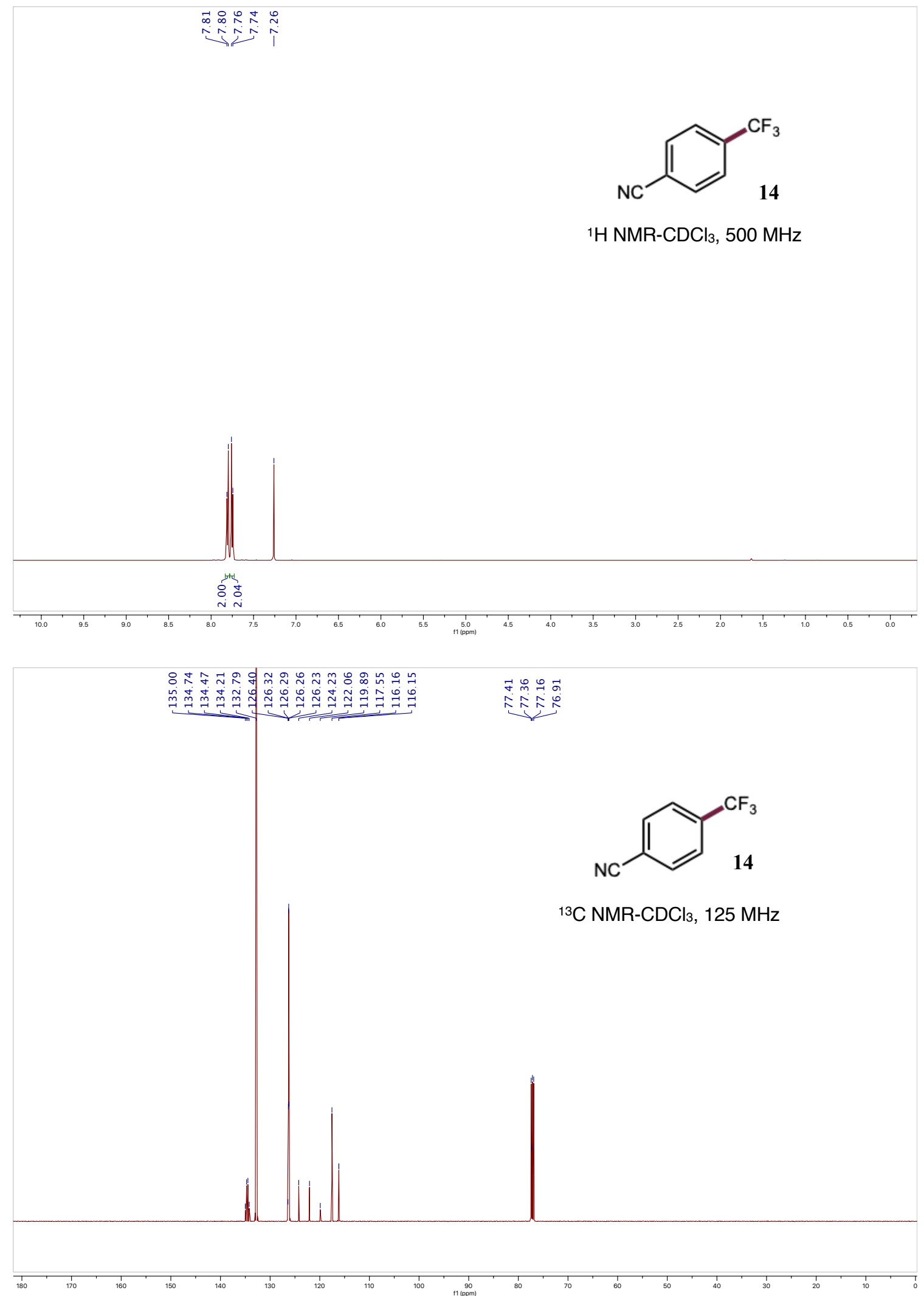


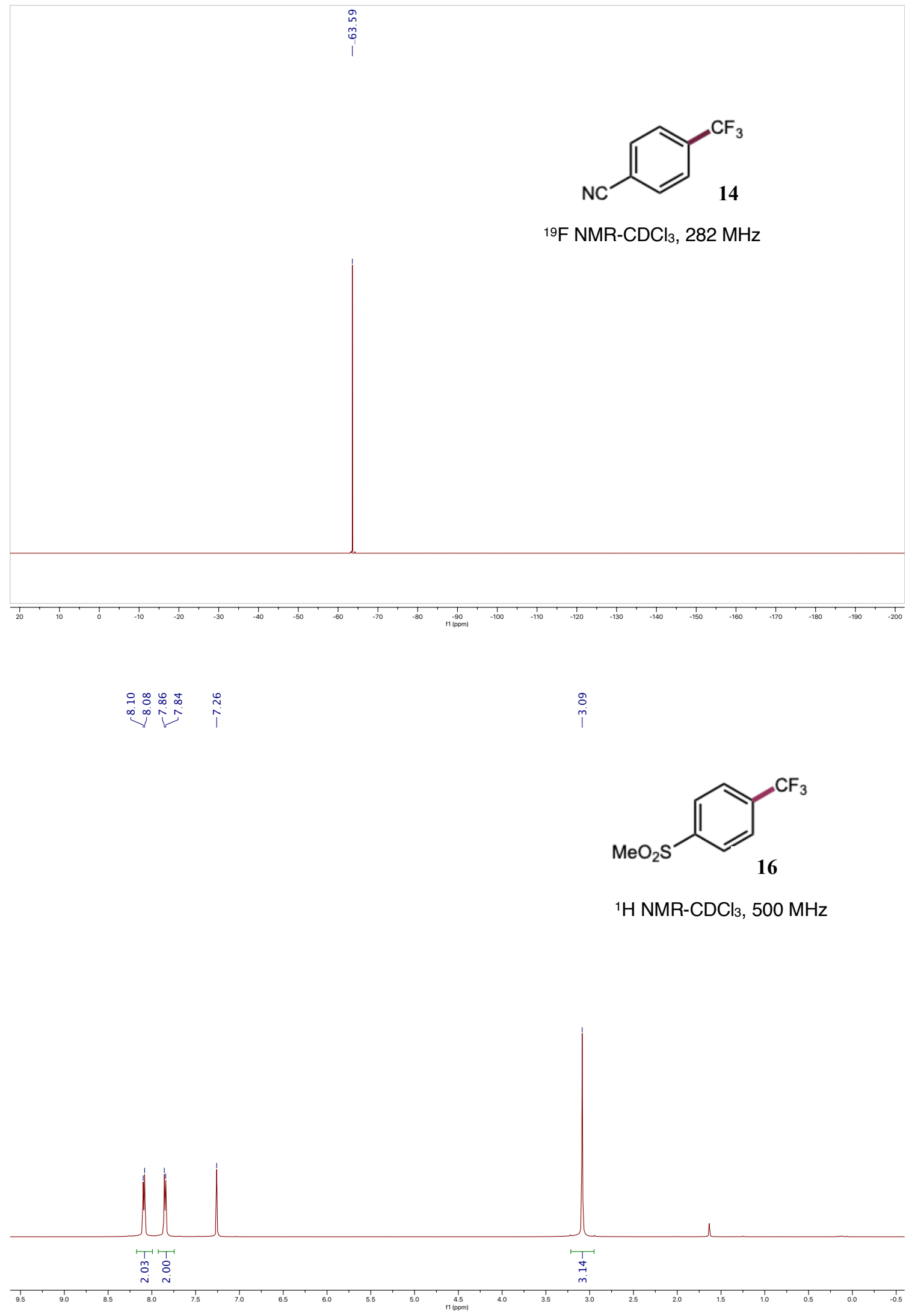



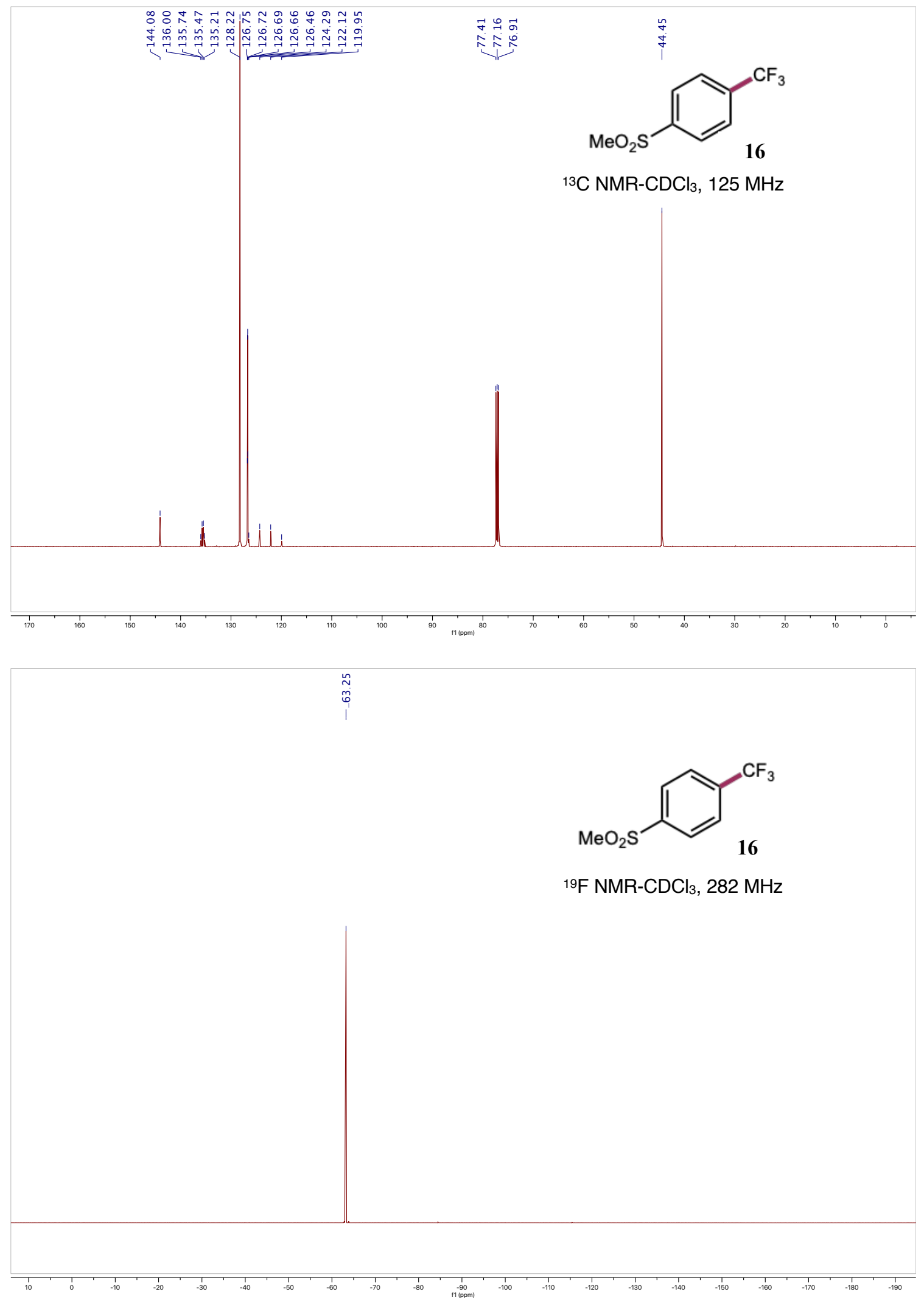


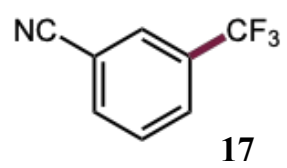

${ }^{1} \mathrm{H} \mathrm{NMR-CDCl} 3,500 \mathrm{MHz}$

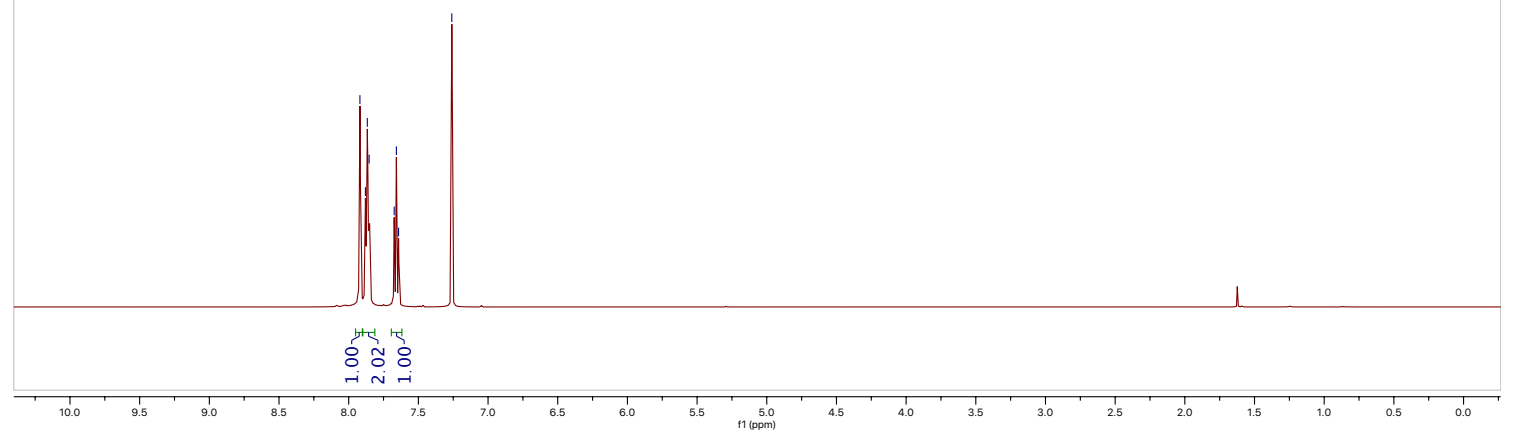

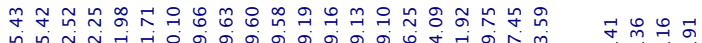

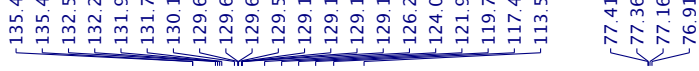
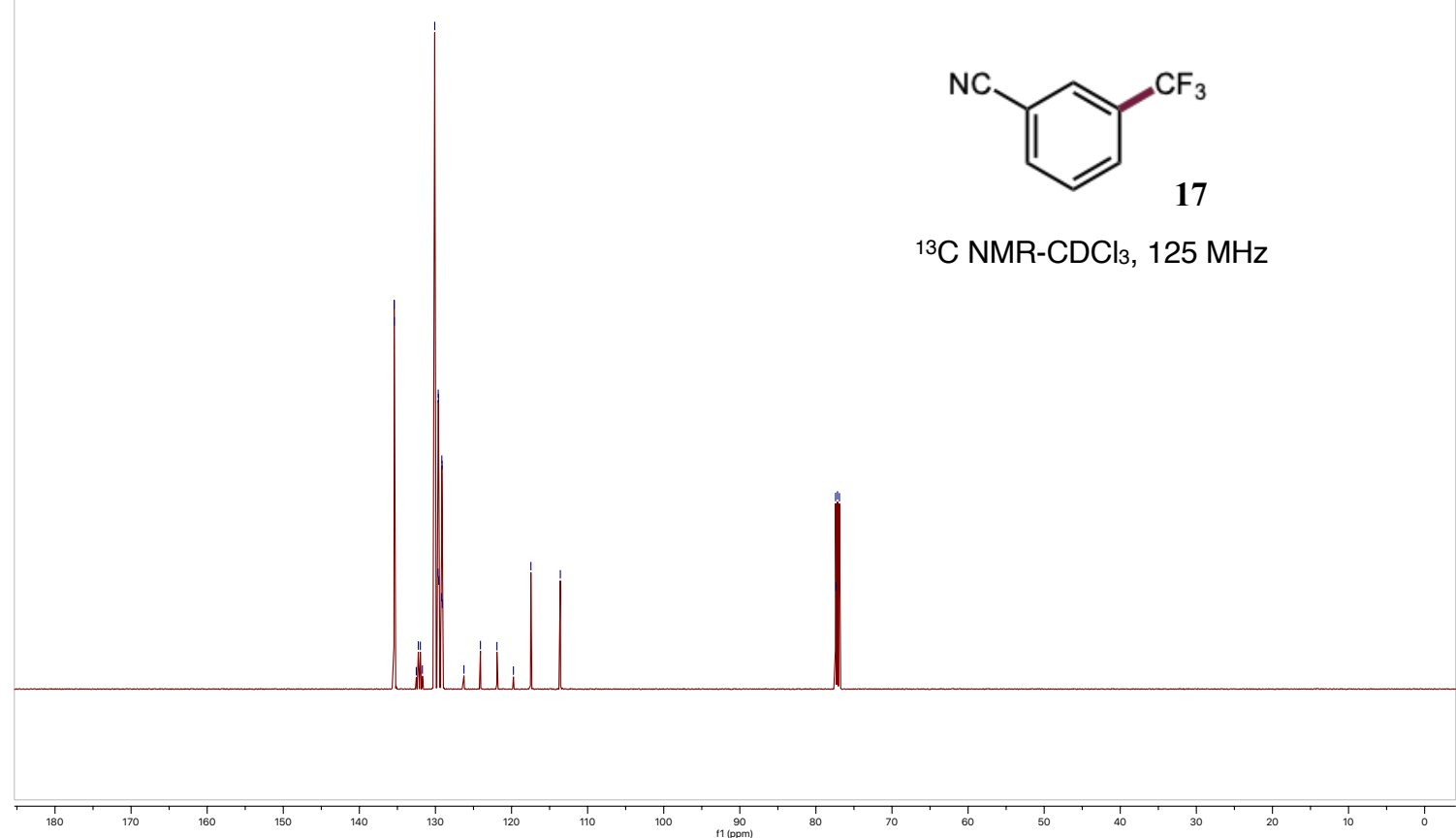


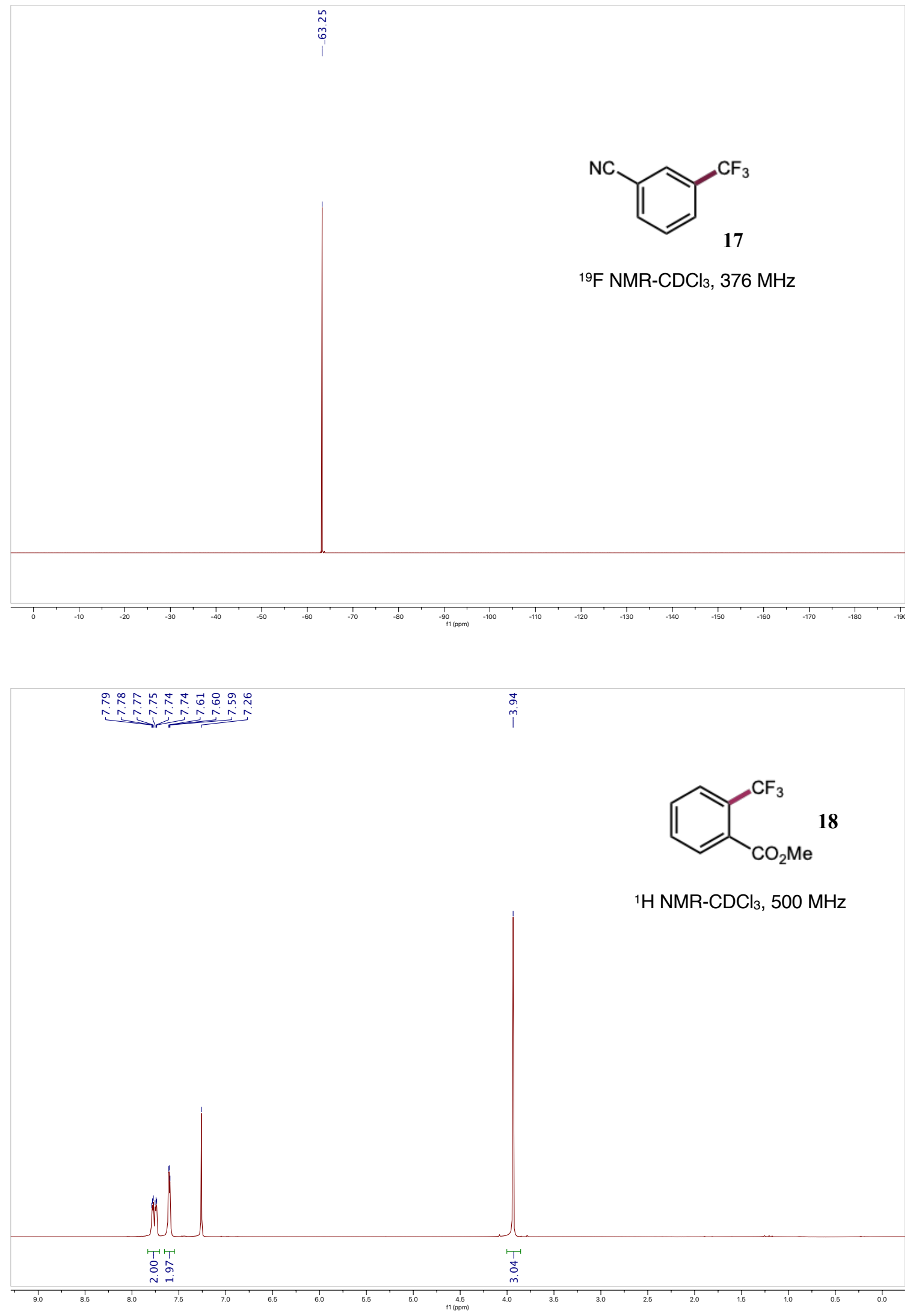



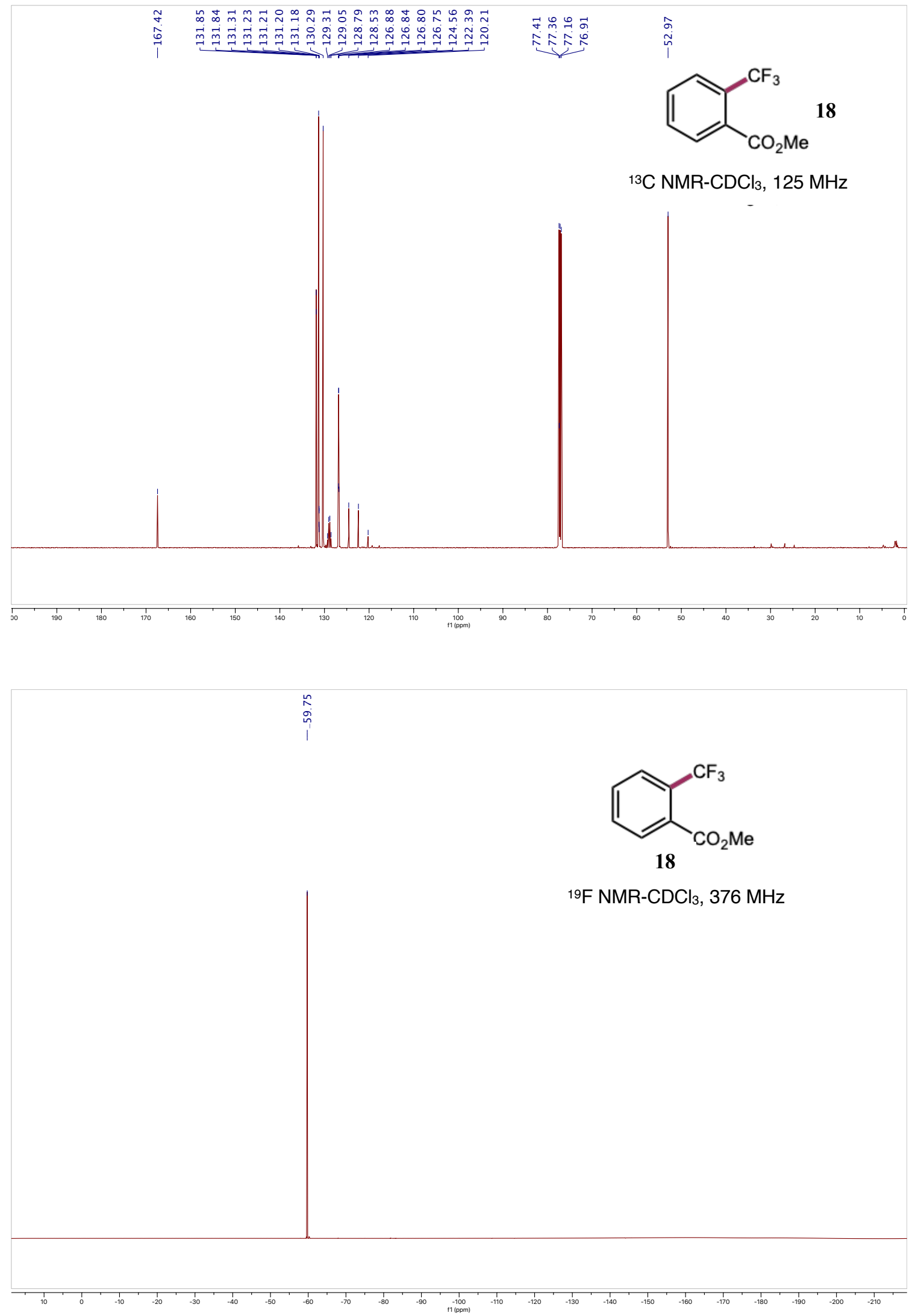


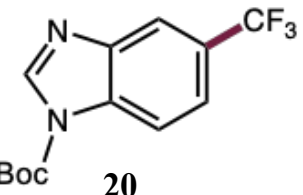

${ }^{1} \mathrm{H}$ NMR $-d_{6}$-DMSO $500 \mathrm{MHz}$

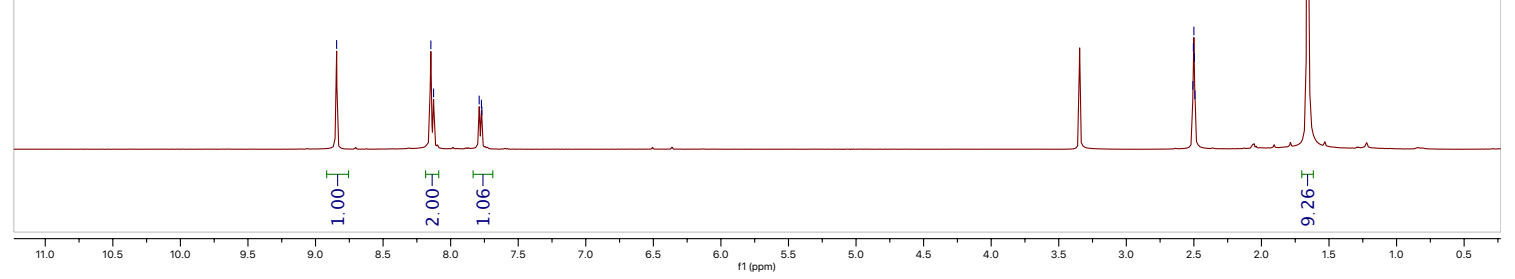

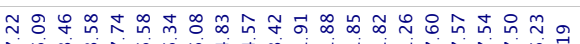

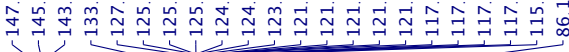

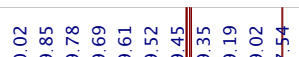

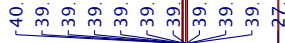<smiles>CC(C)(C)OC(=O)n1cnc2cc(C(F)(F)F)ccc21</smiles>

${ }^{13} \mathrm{C}$ NMR $-d_{6}$-DMSO, $125 \mathrm{MHz}$

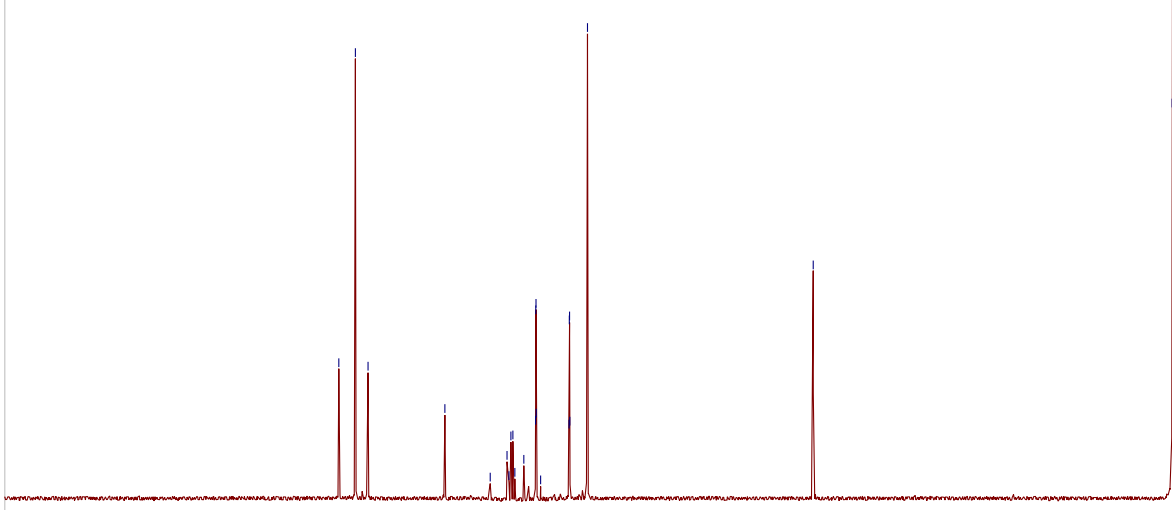



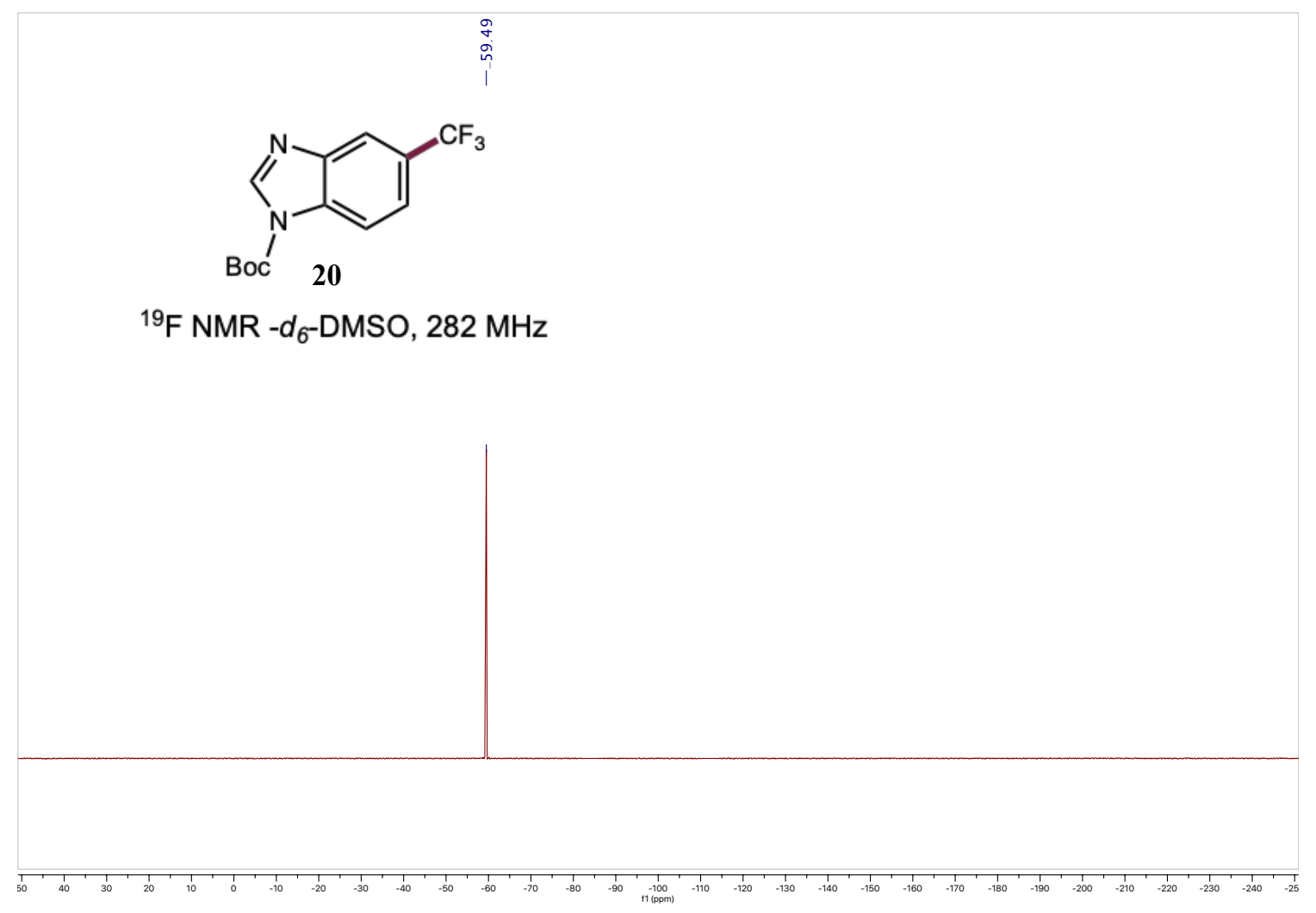

$\underbrace{0}_{0}$

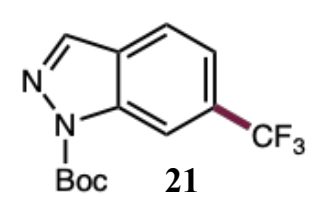

${ }^{1} \mathrm{H}$ NMR-CDCl $3,500 \mathrm{MHz}$

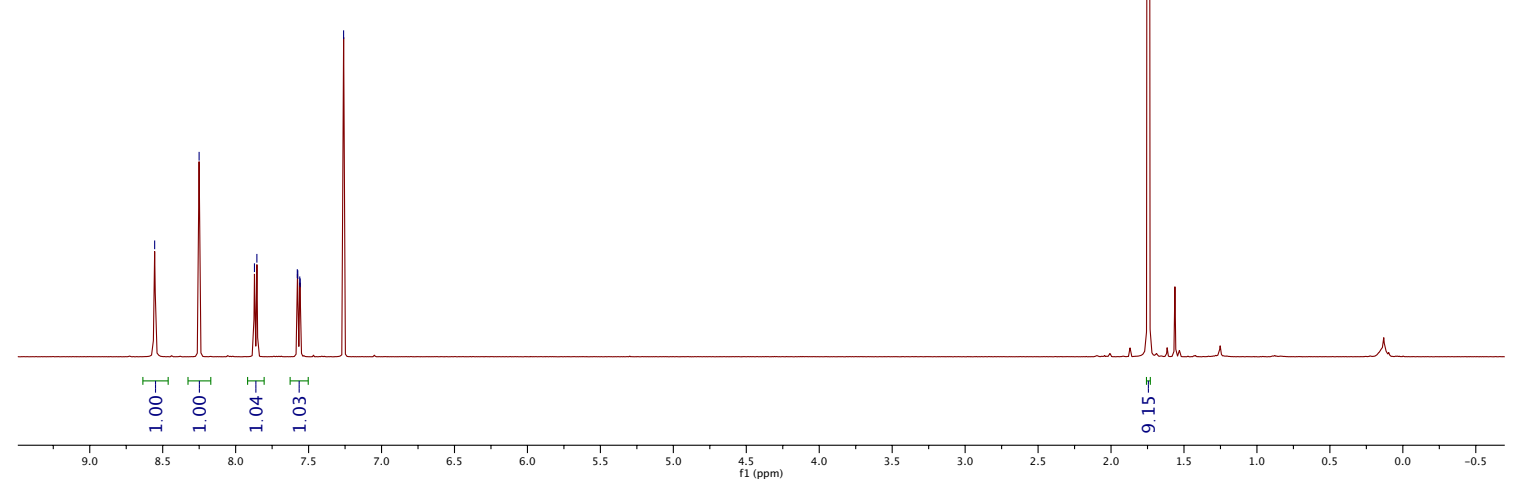



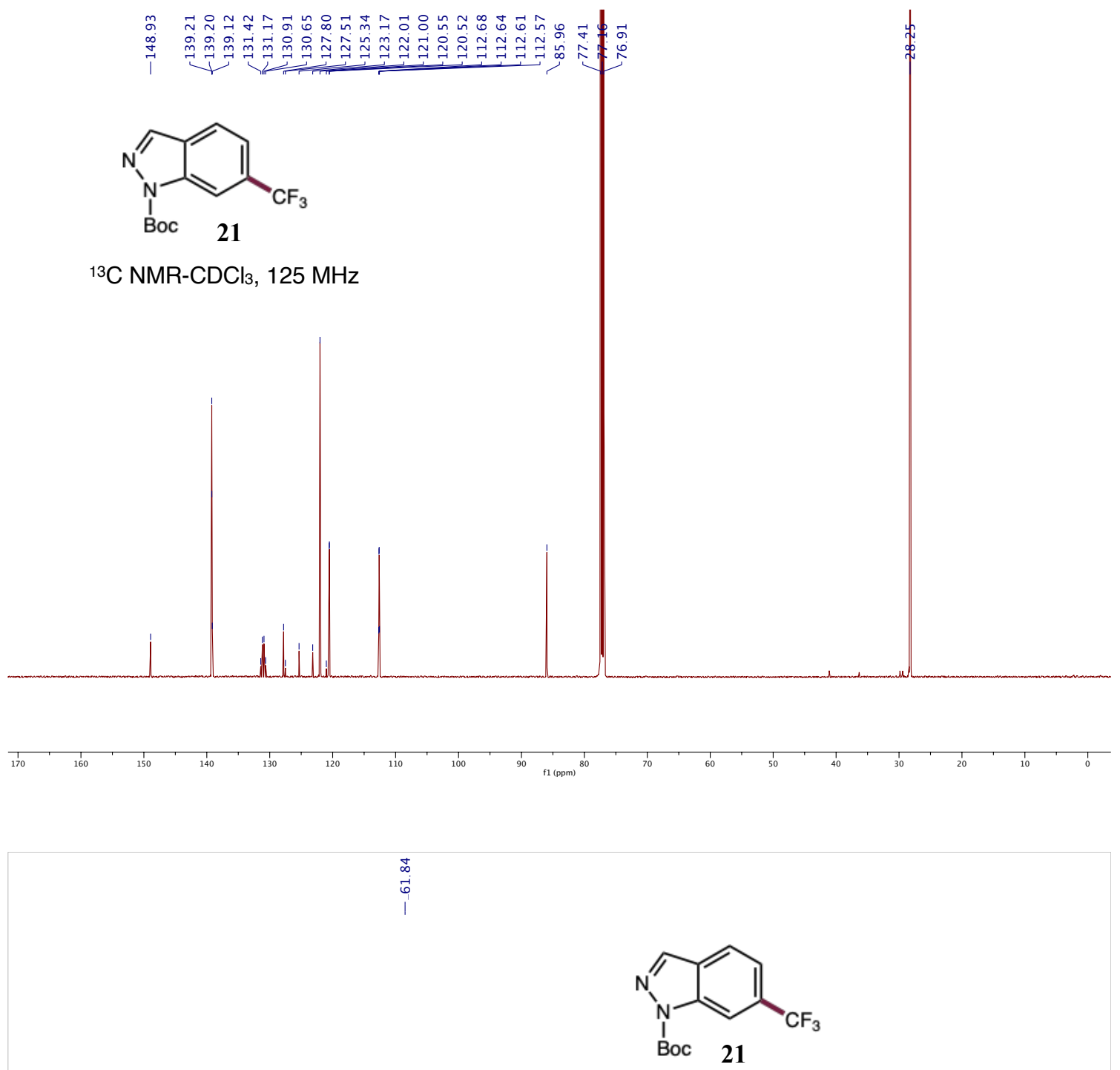

19F NMR-CDCl $3,376 \mathrm{MHz}$
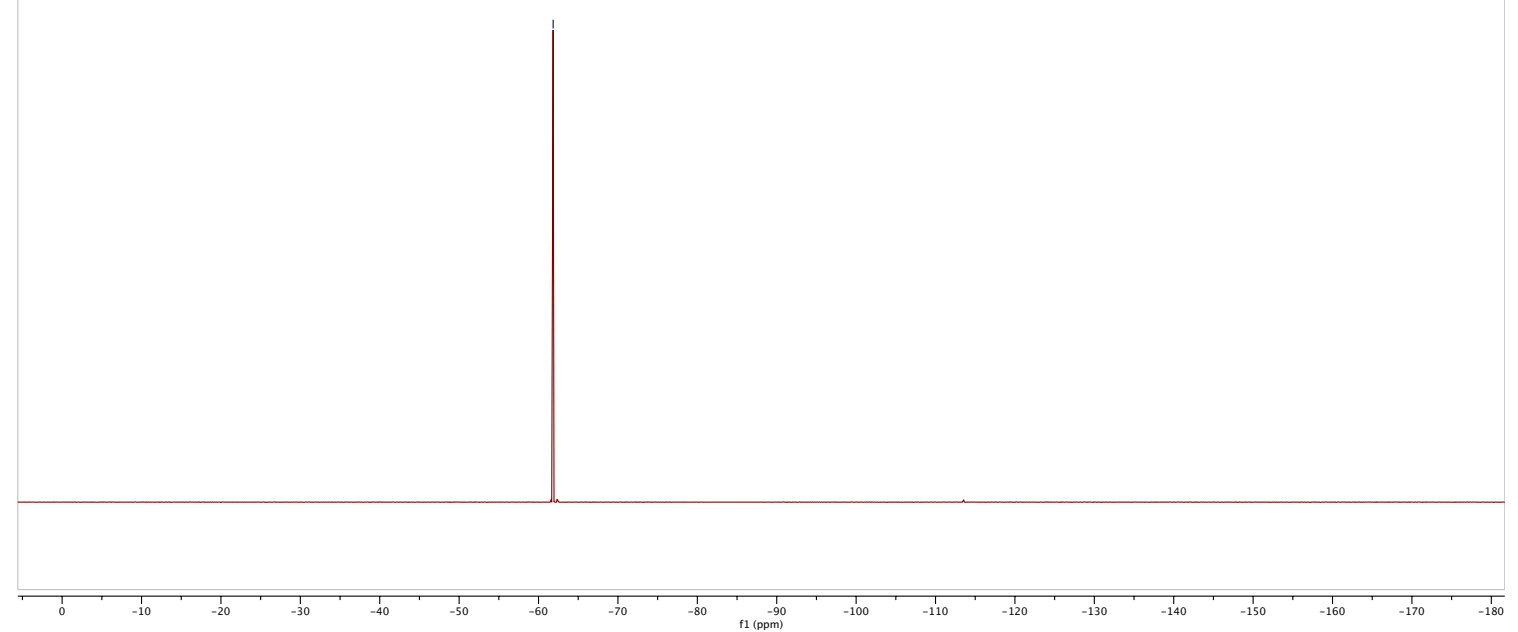

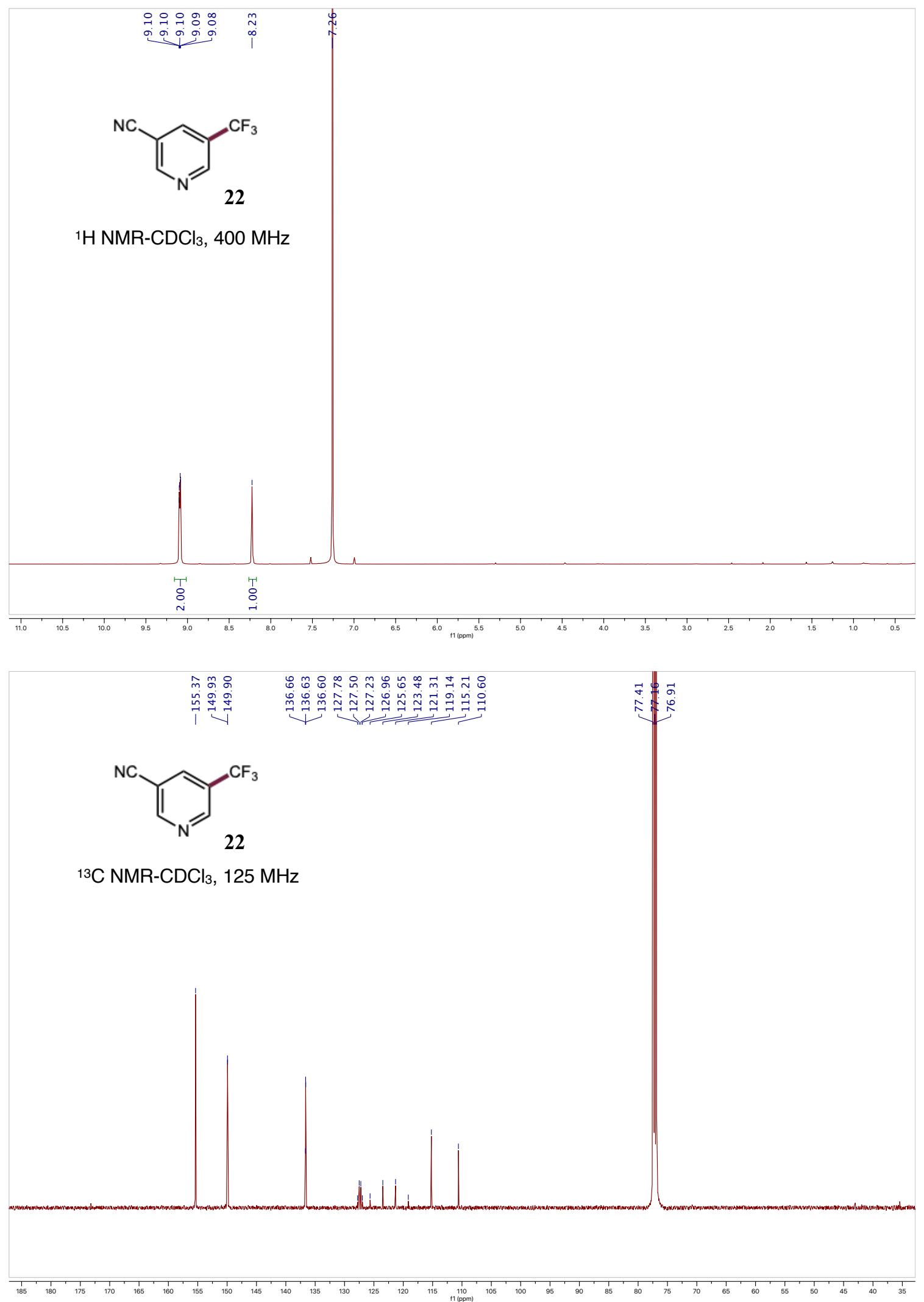


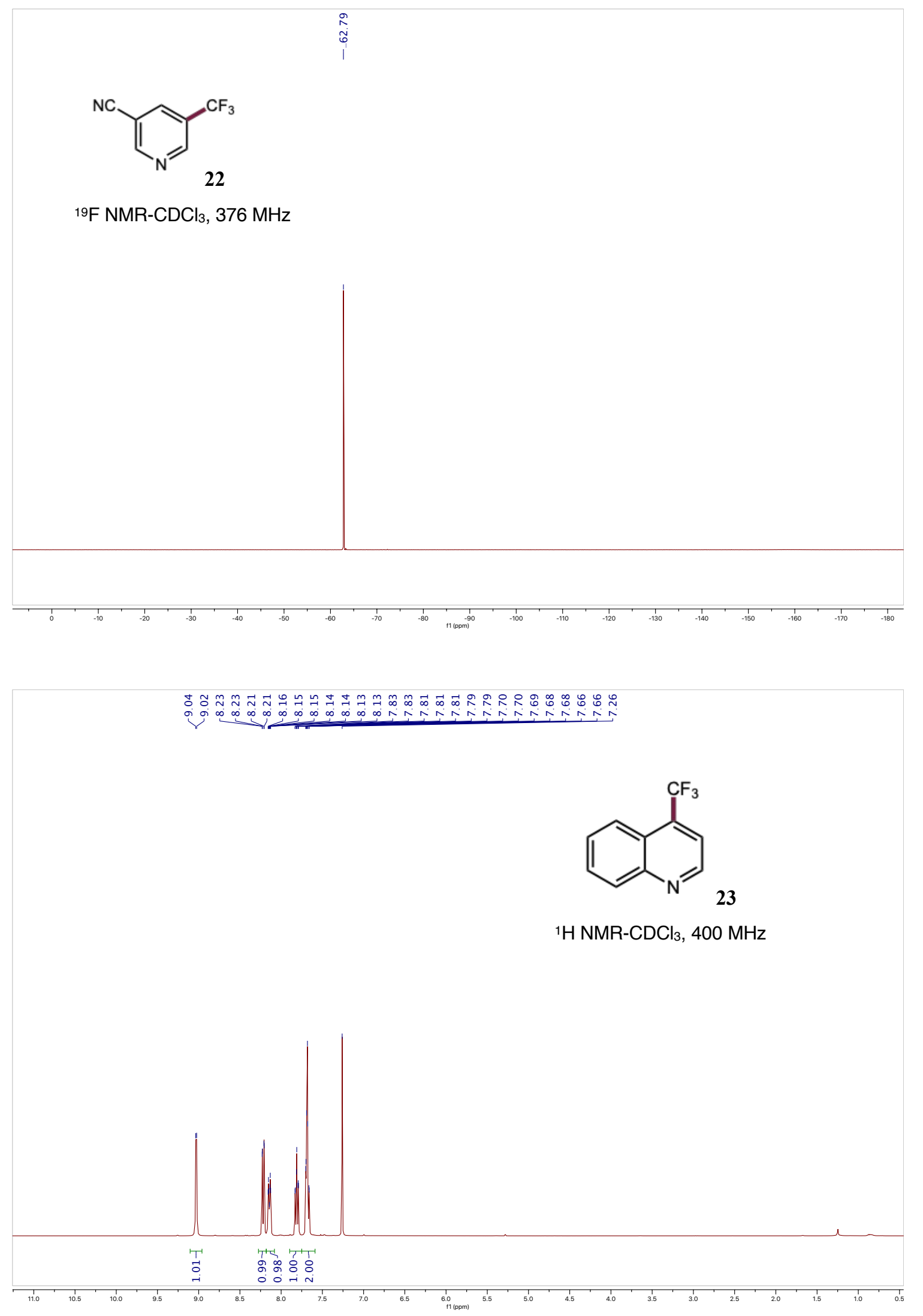



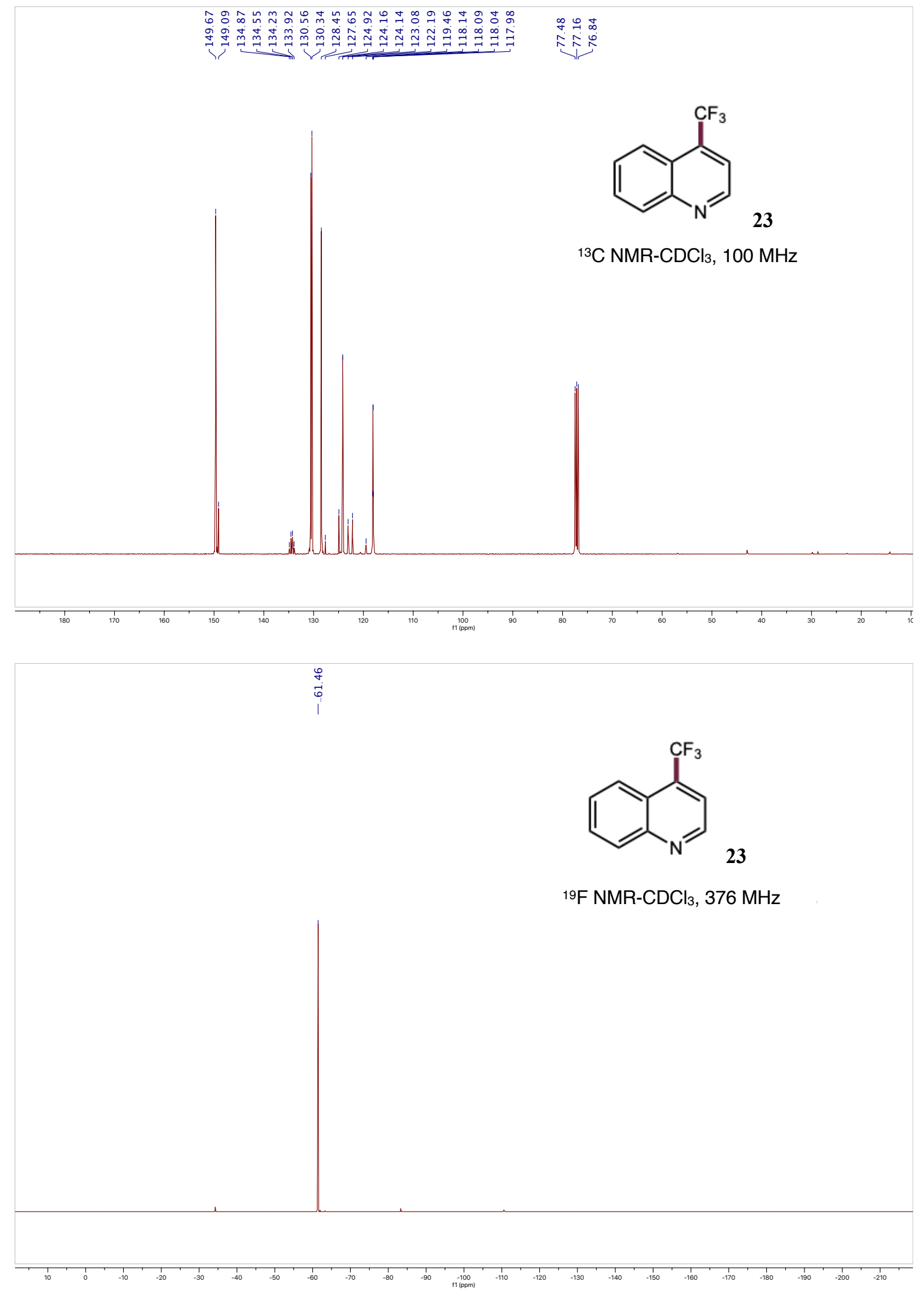

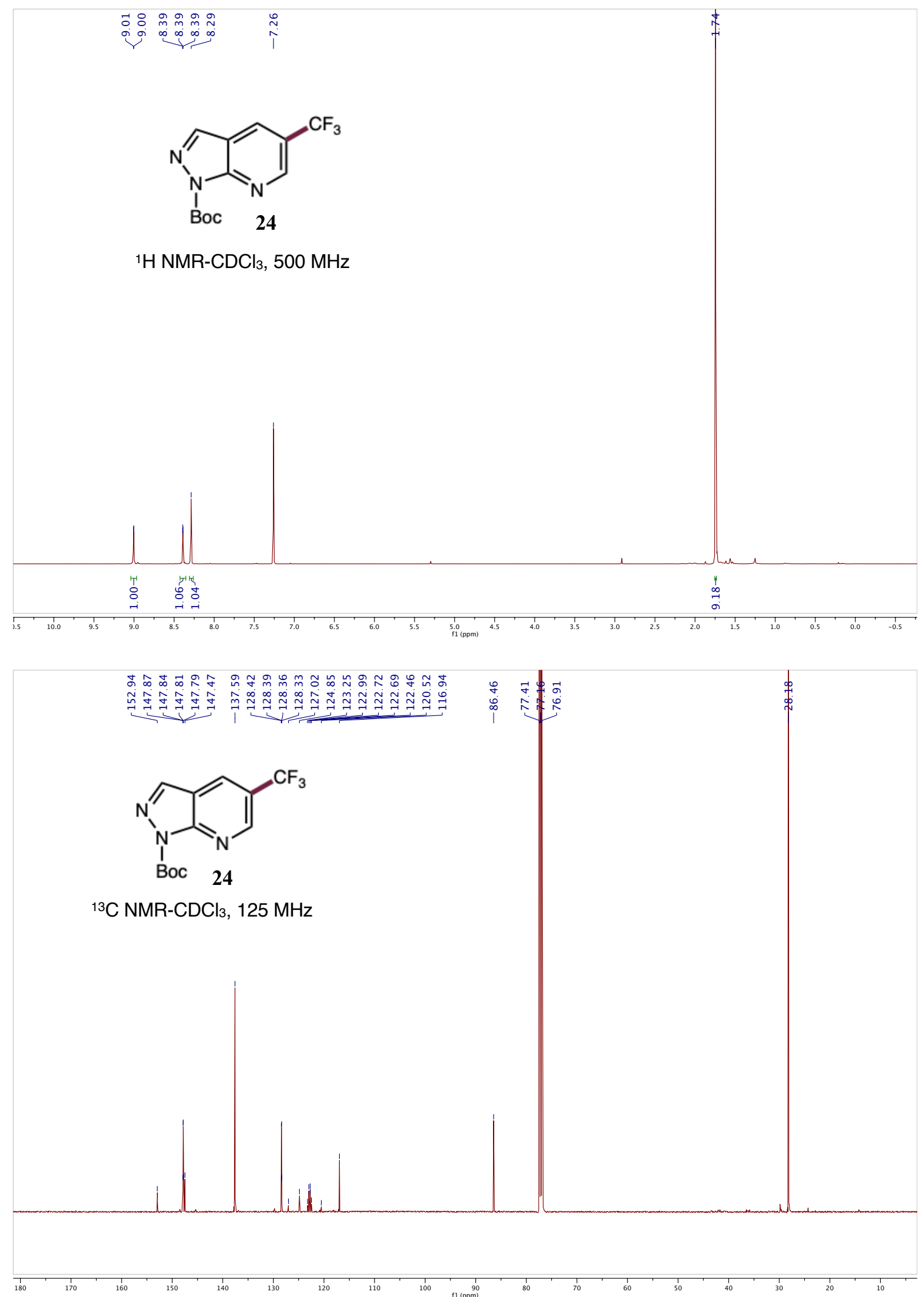

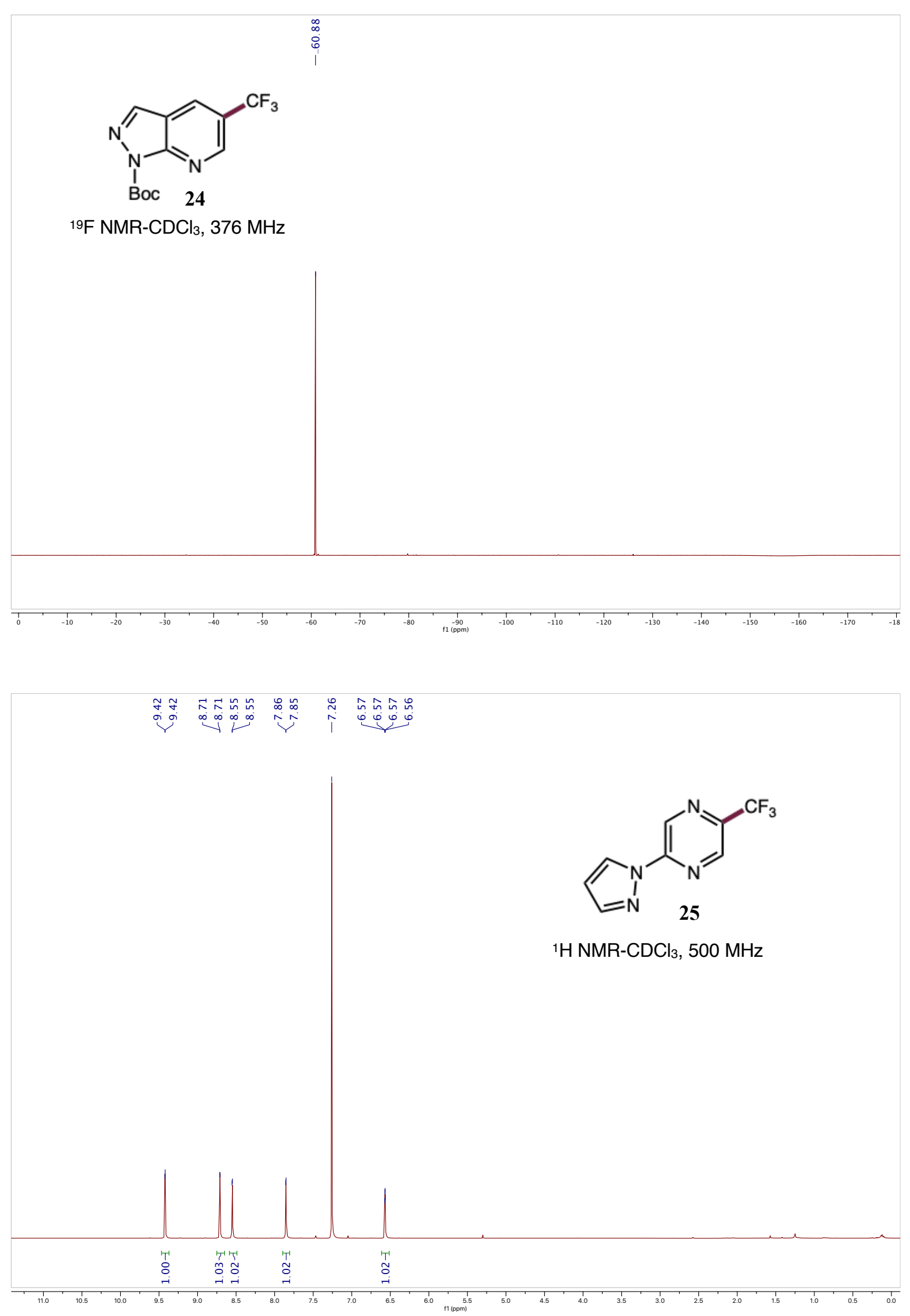

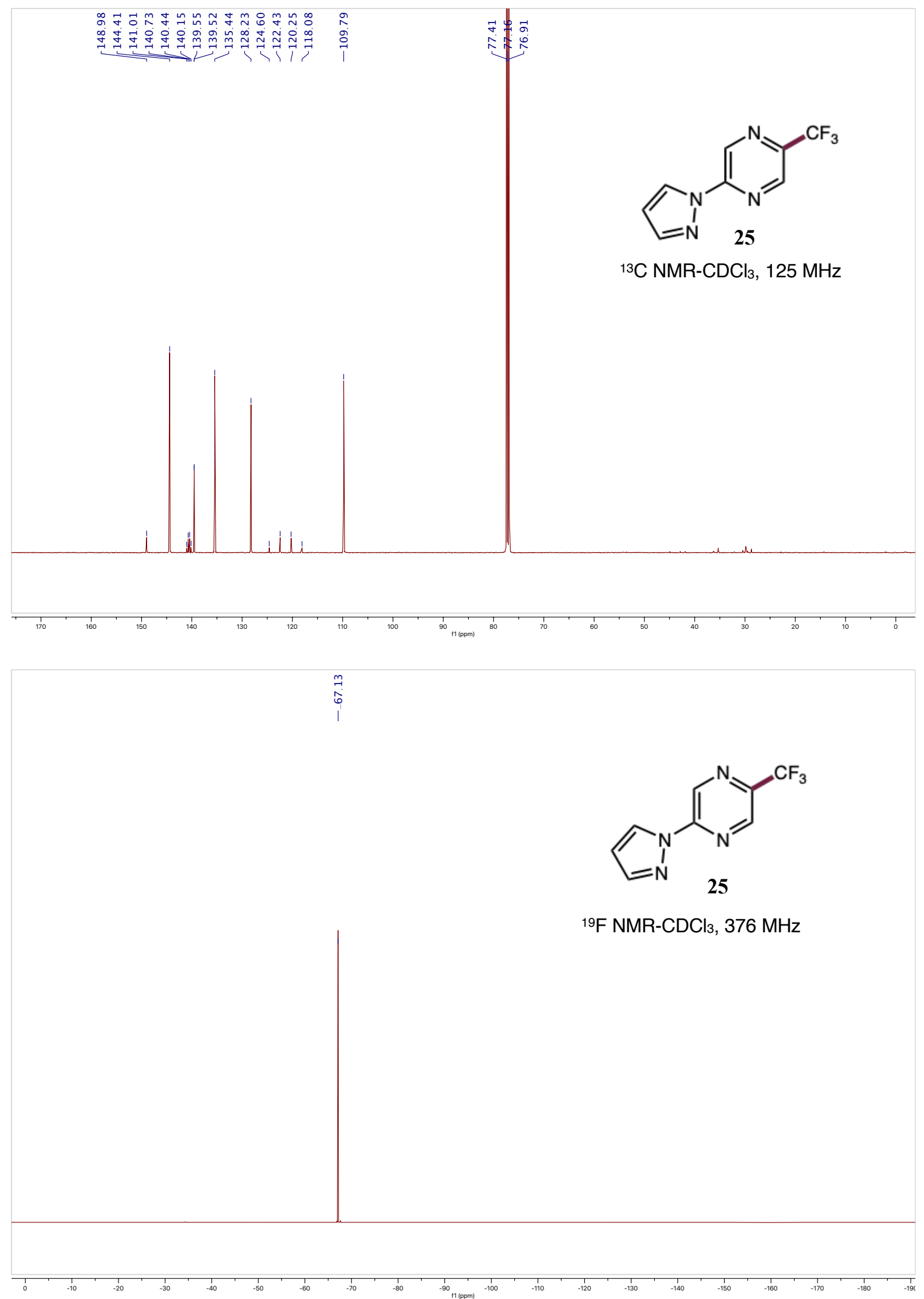


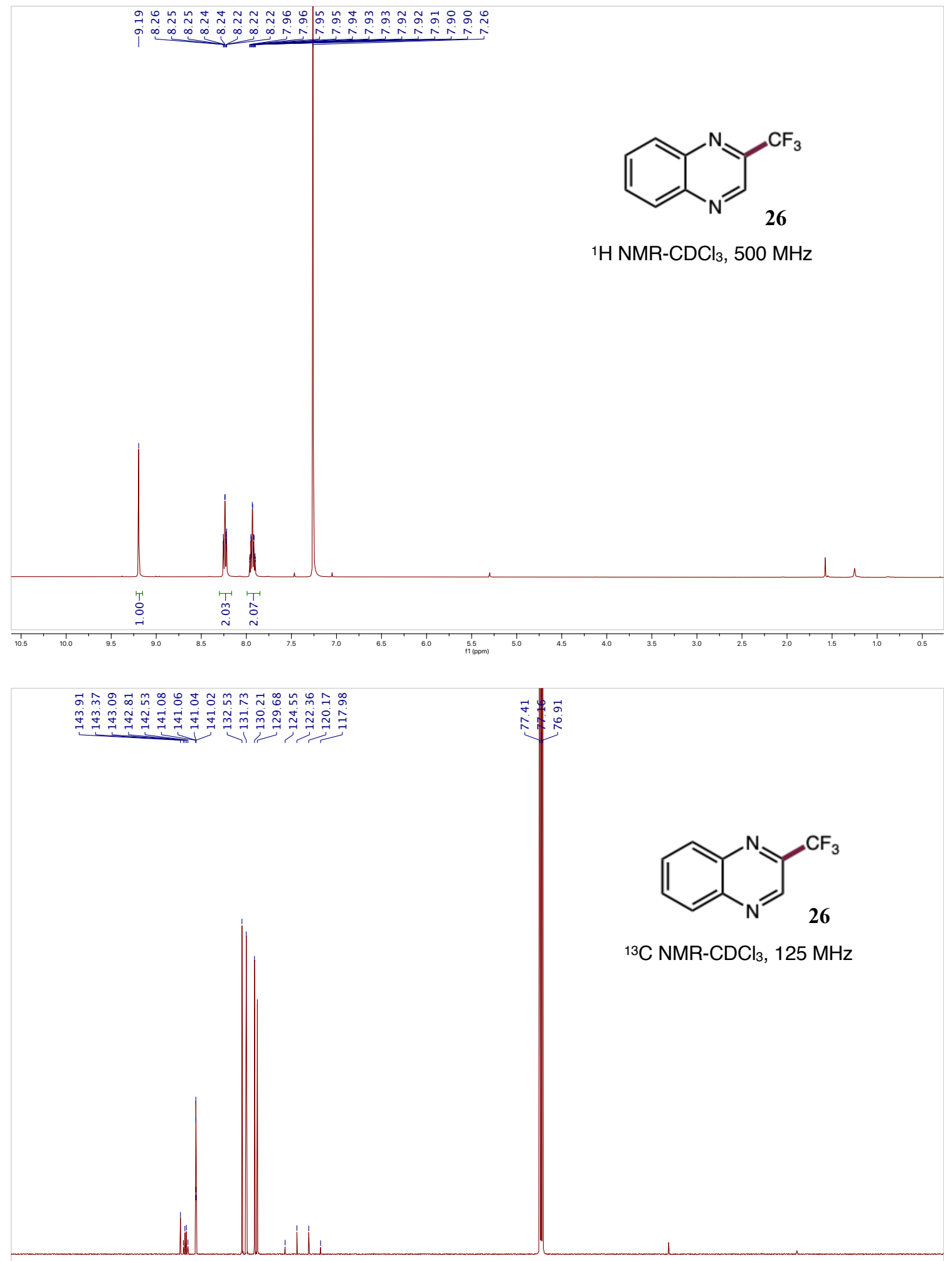




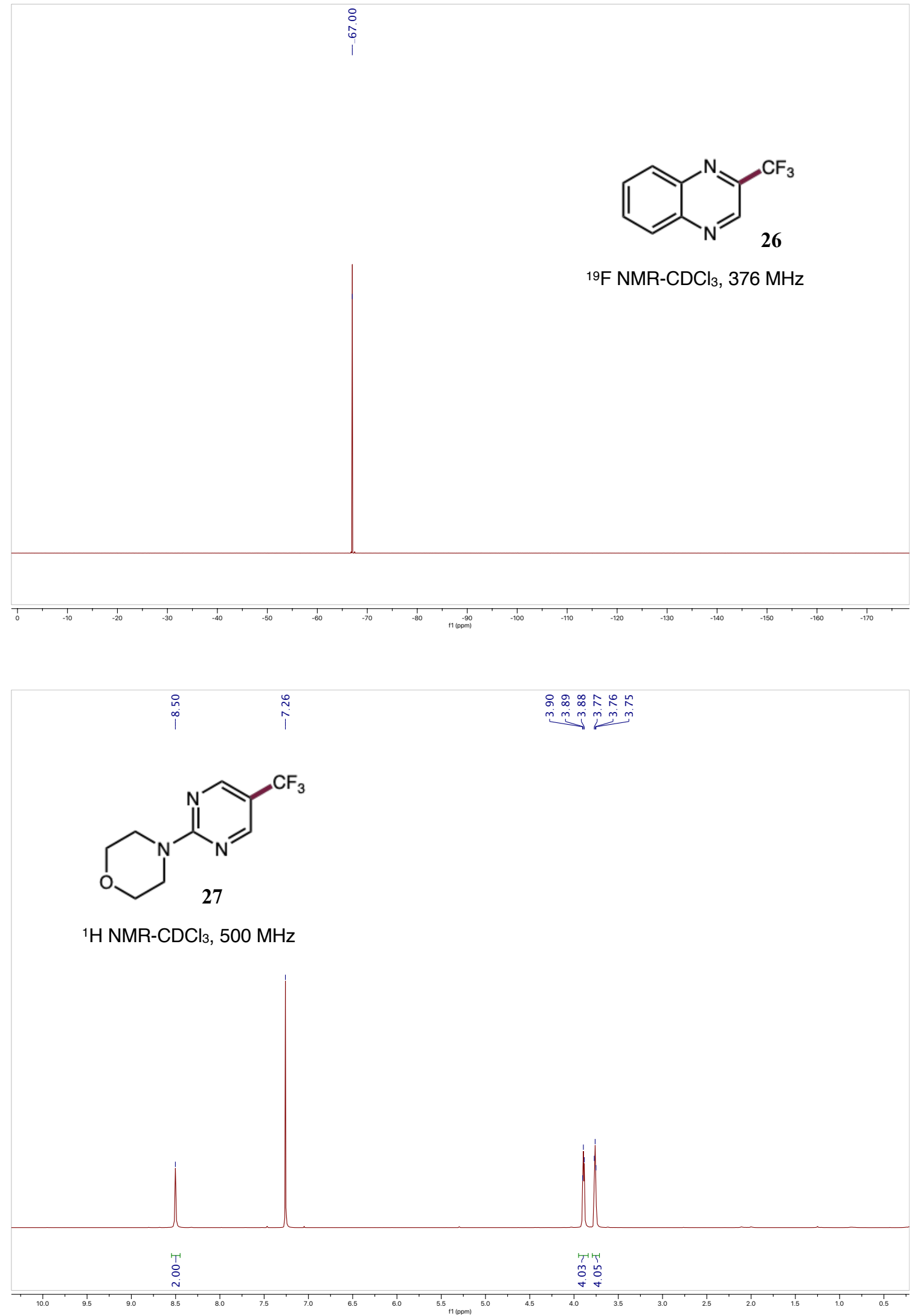



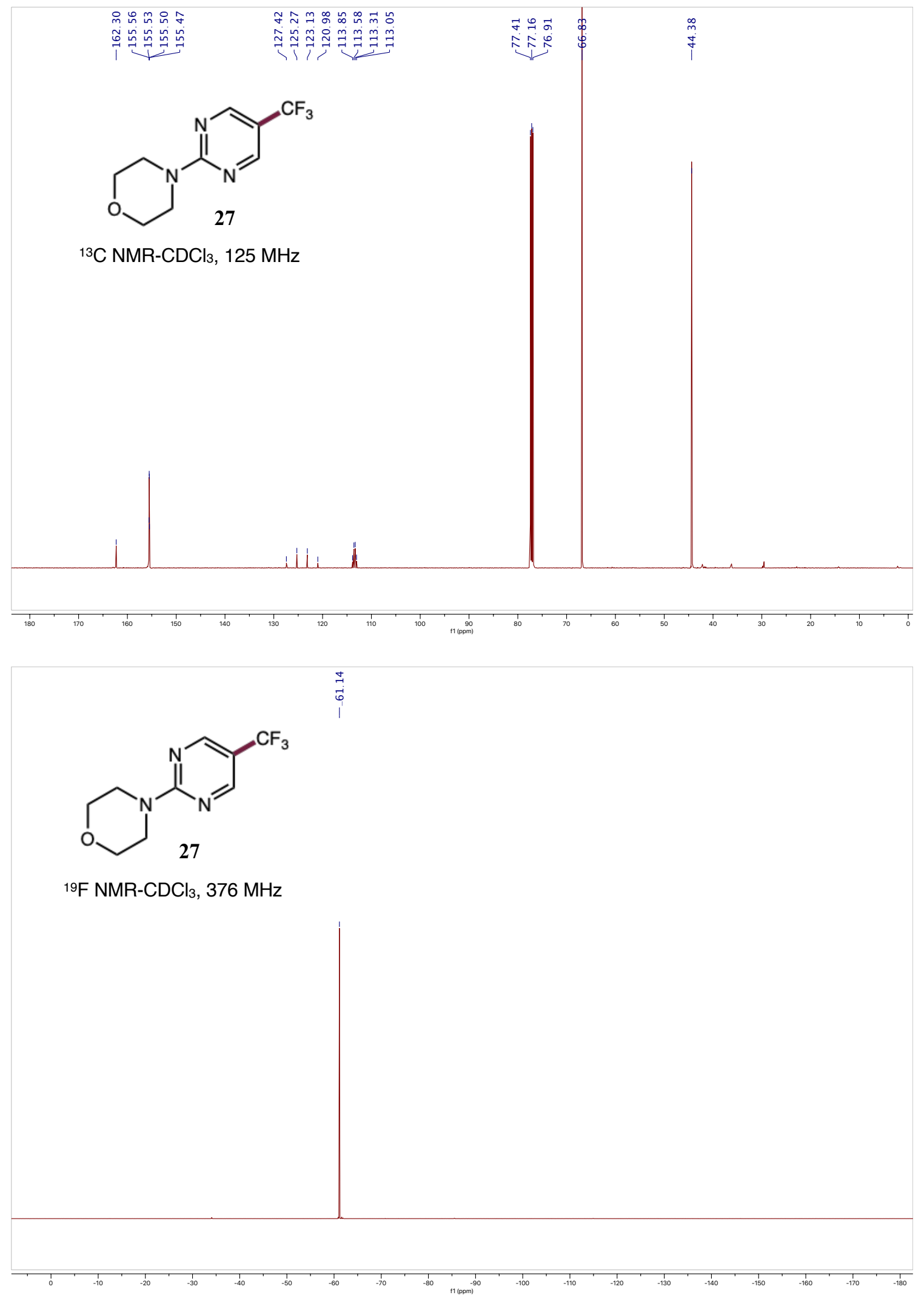

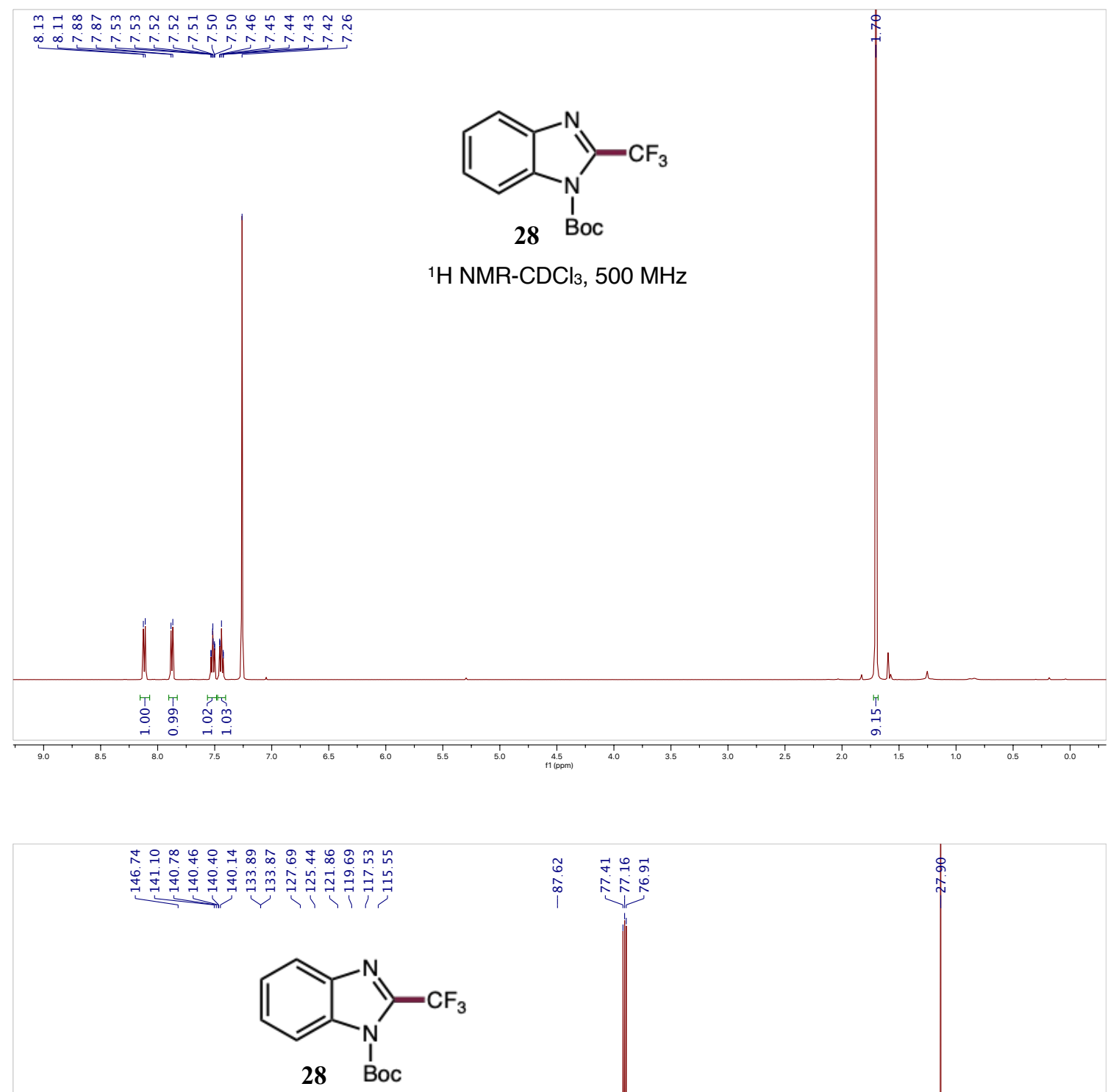

${ }^{13} \mathrm{C} \mathrm{NMR-CDCl} 3,125 \mathrm{MHz}$
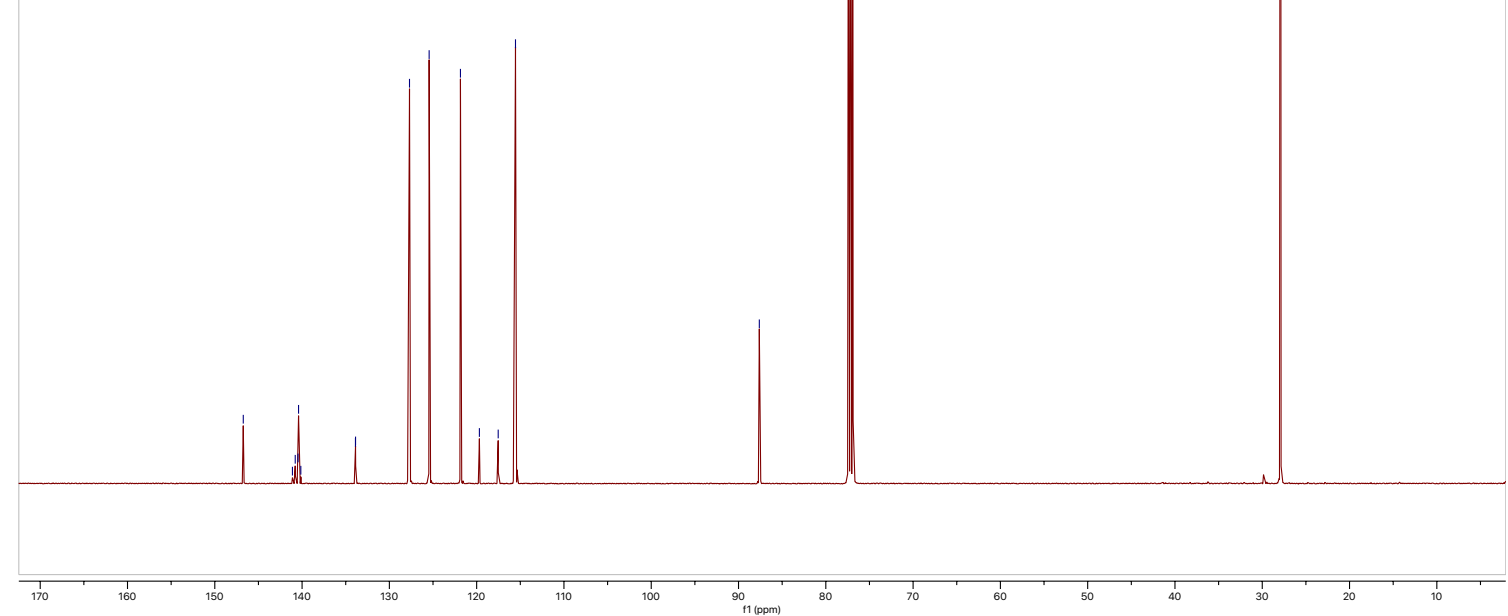

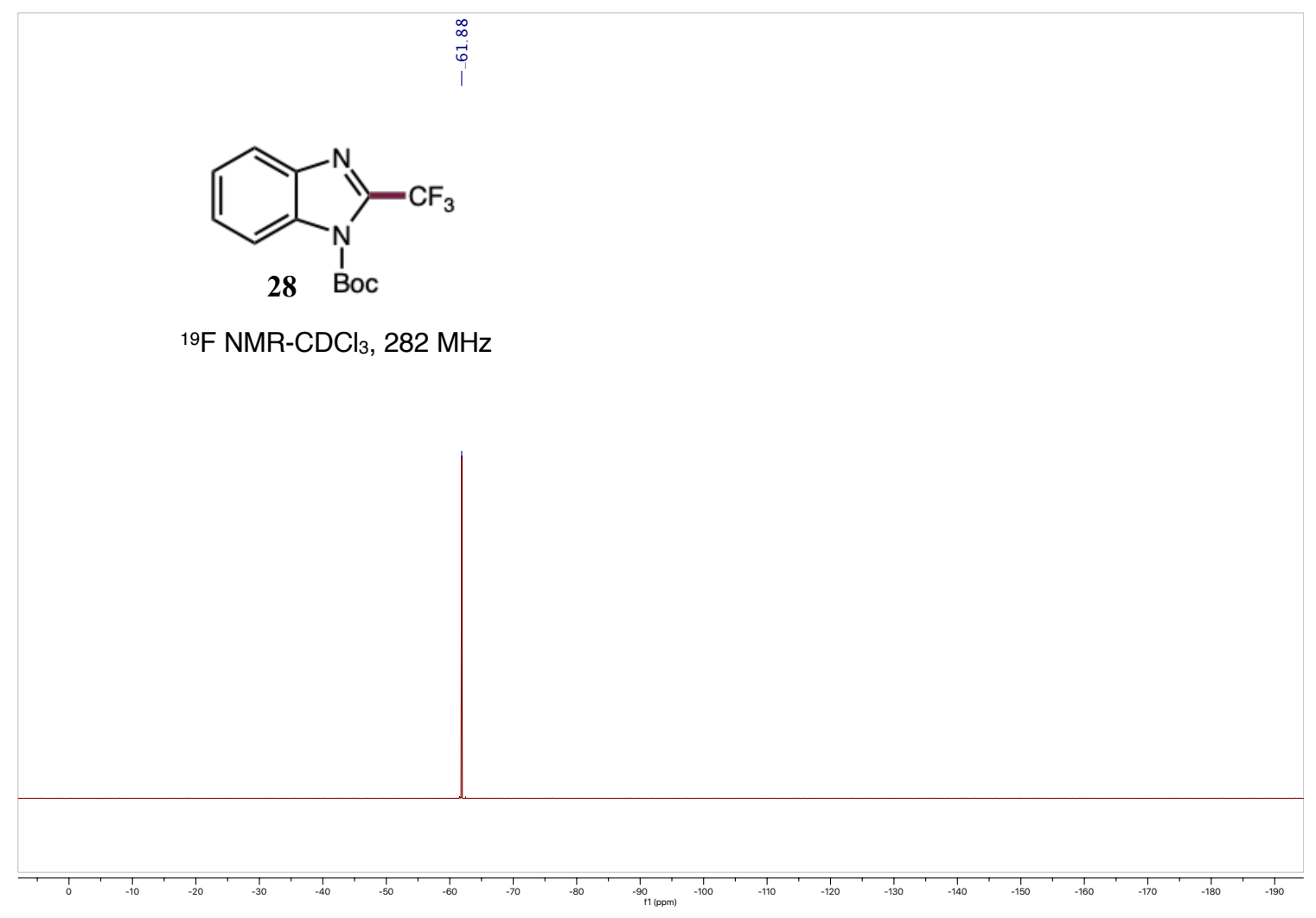

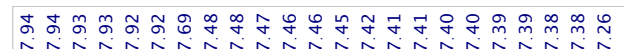

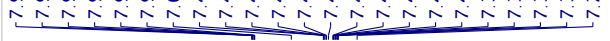

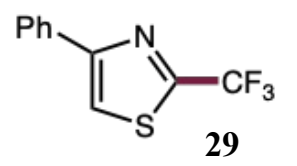

${ }^{1} \mathrm{H} \mathrm{NMR-CDCl} 3,500 \mathrm{MHz}$

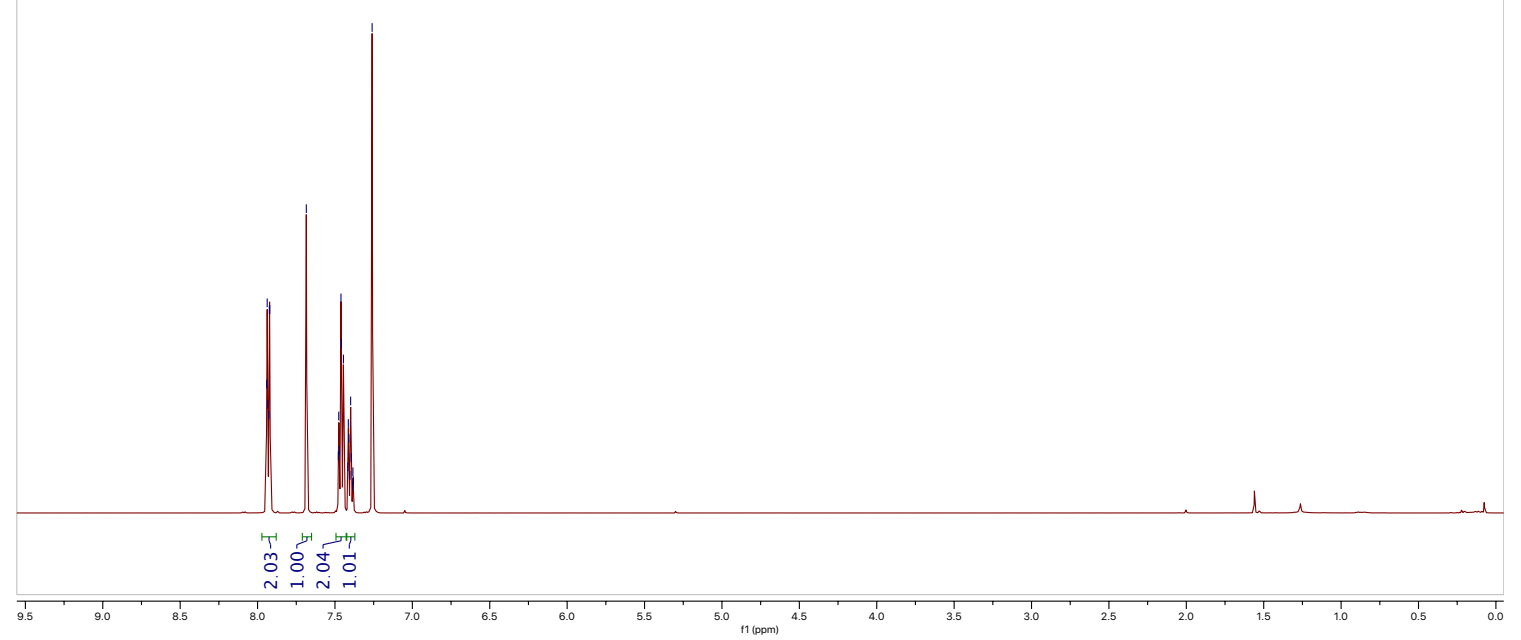



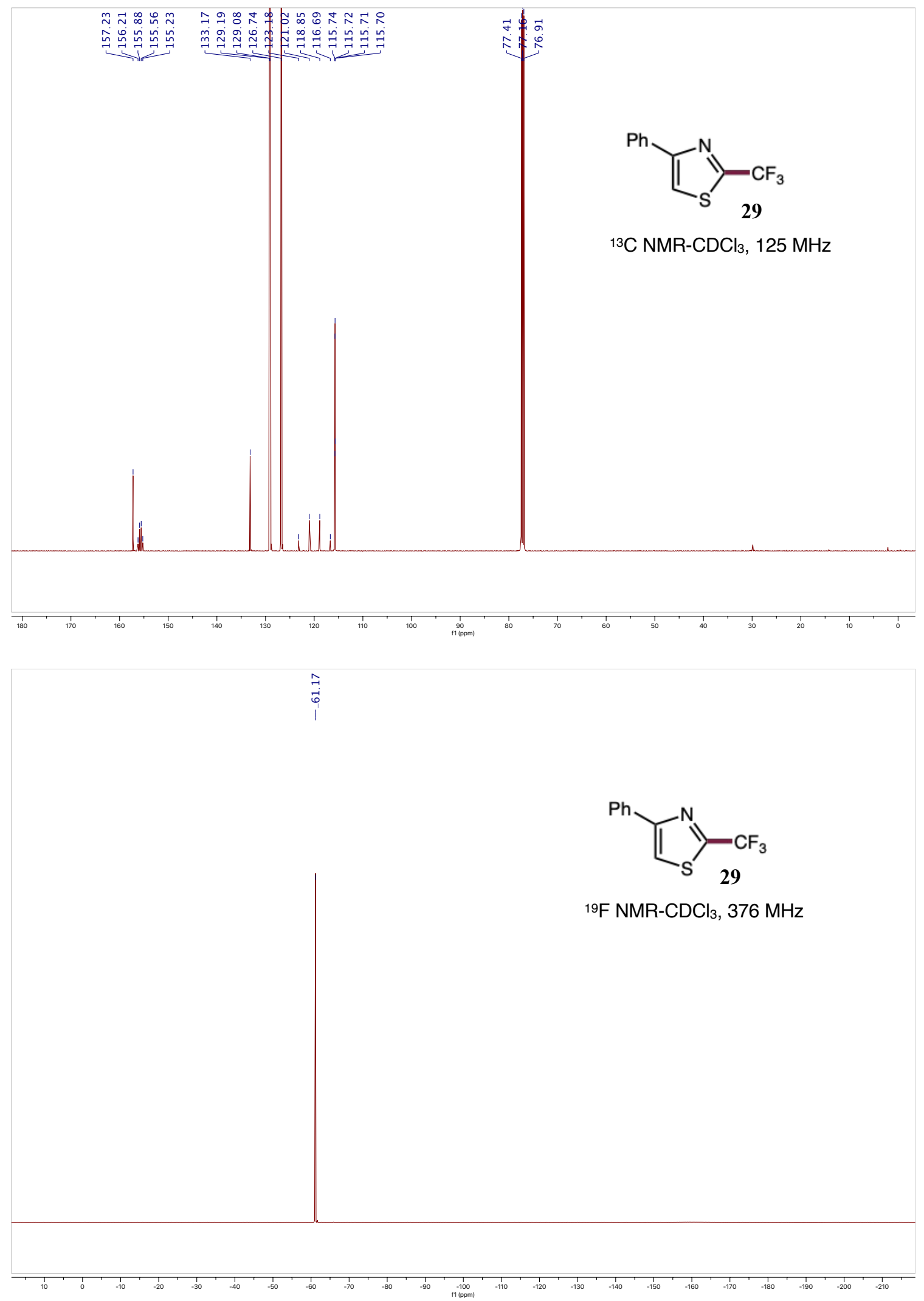

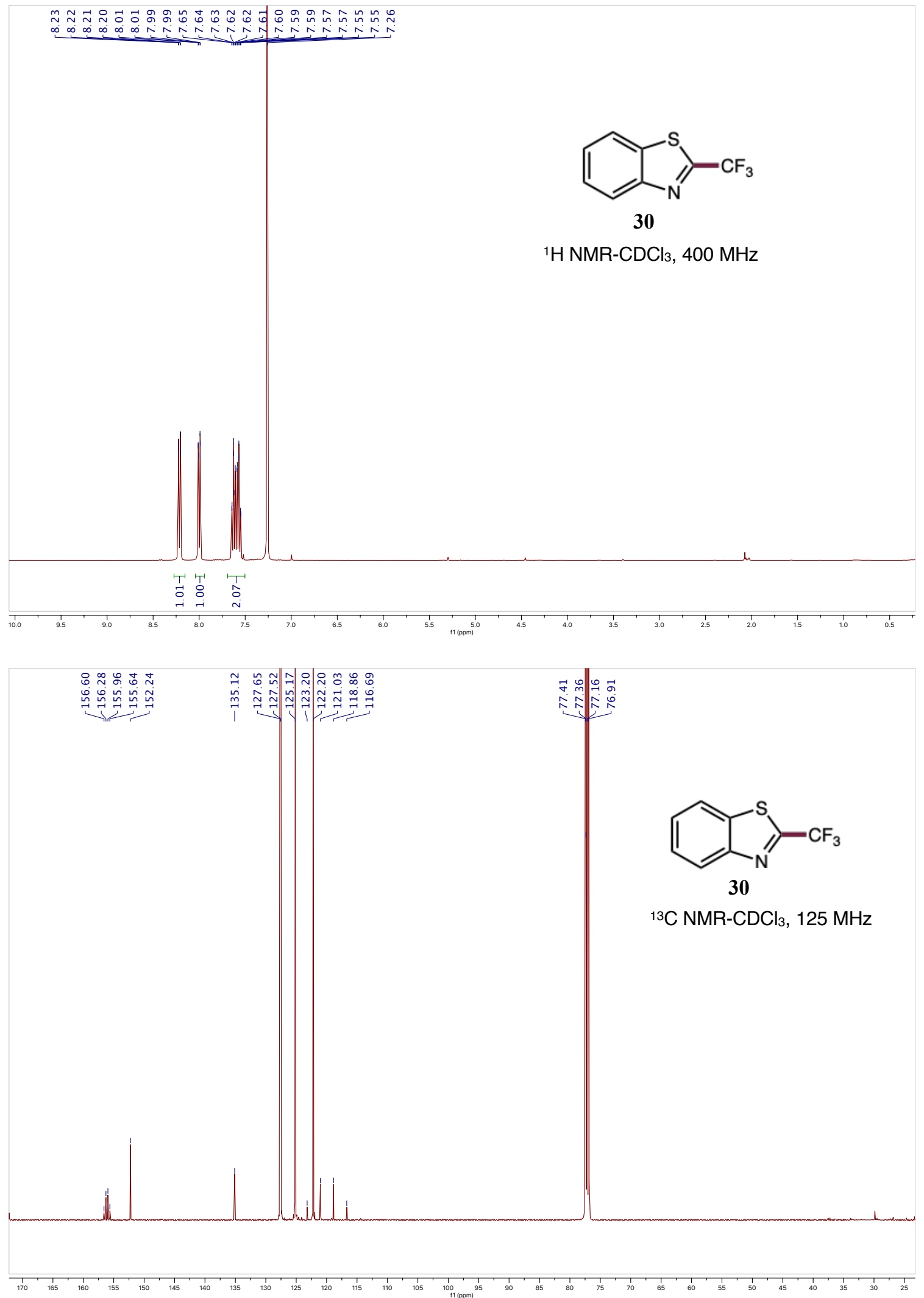


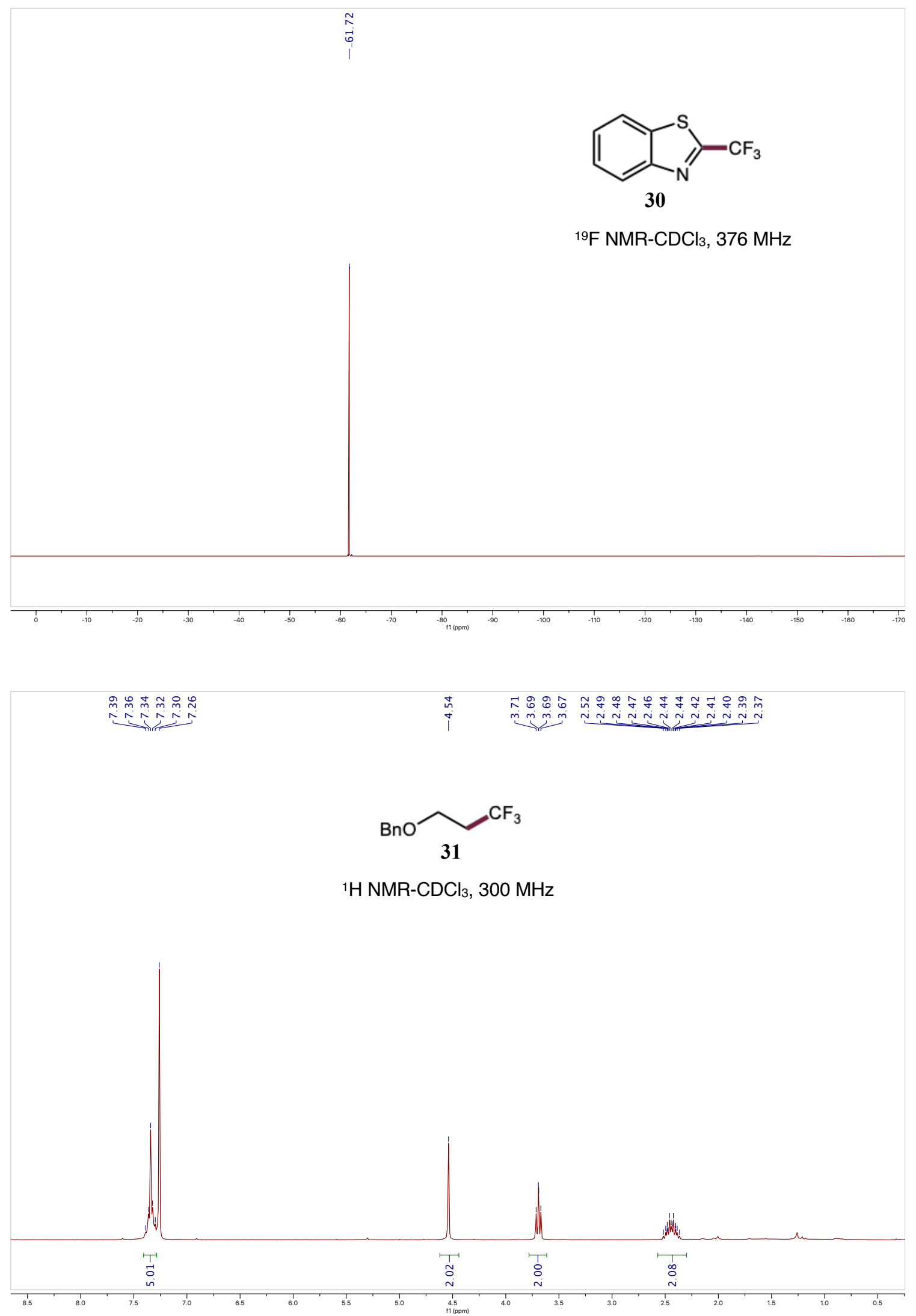



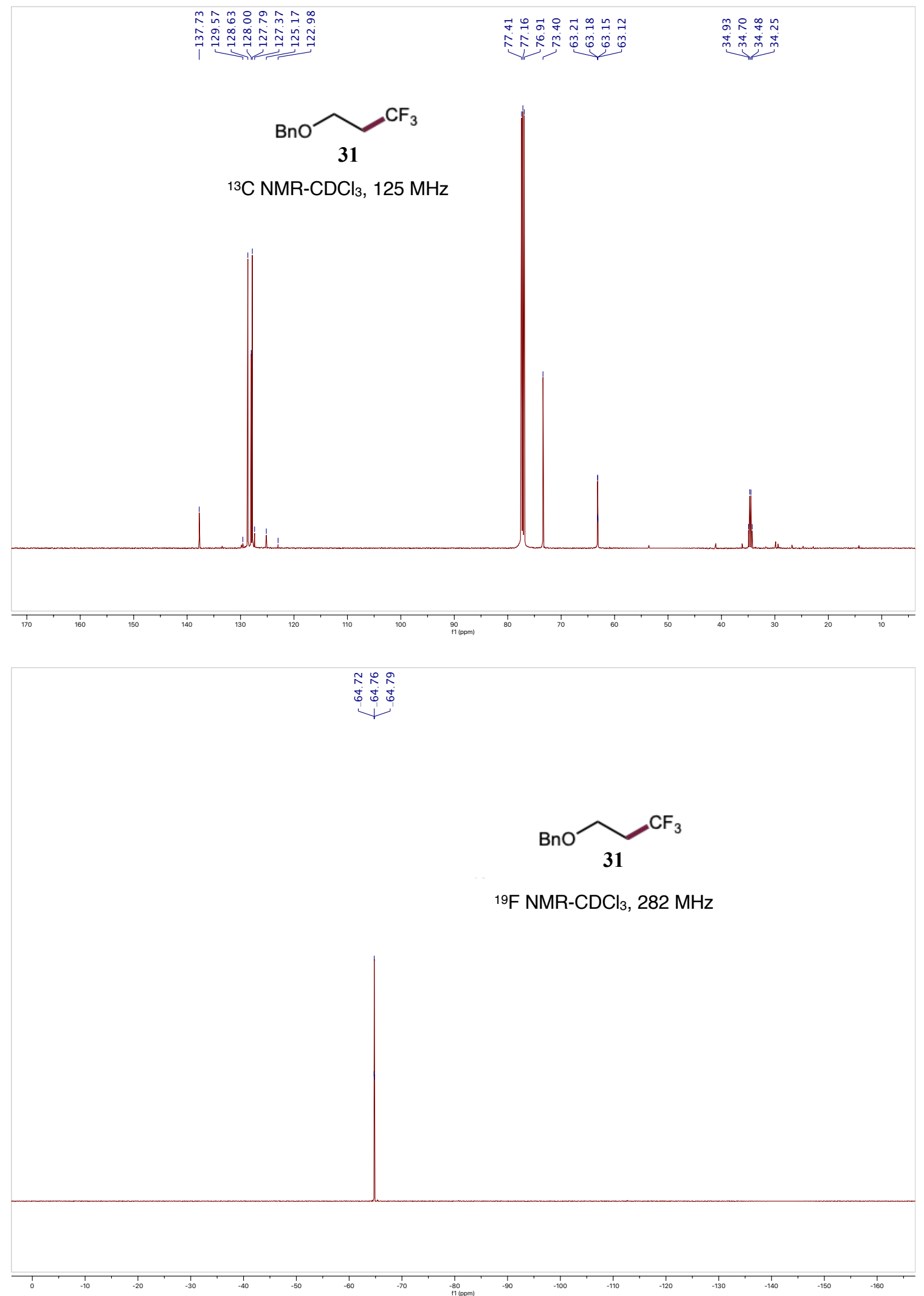

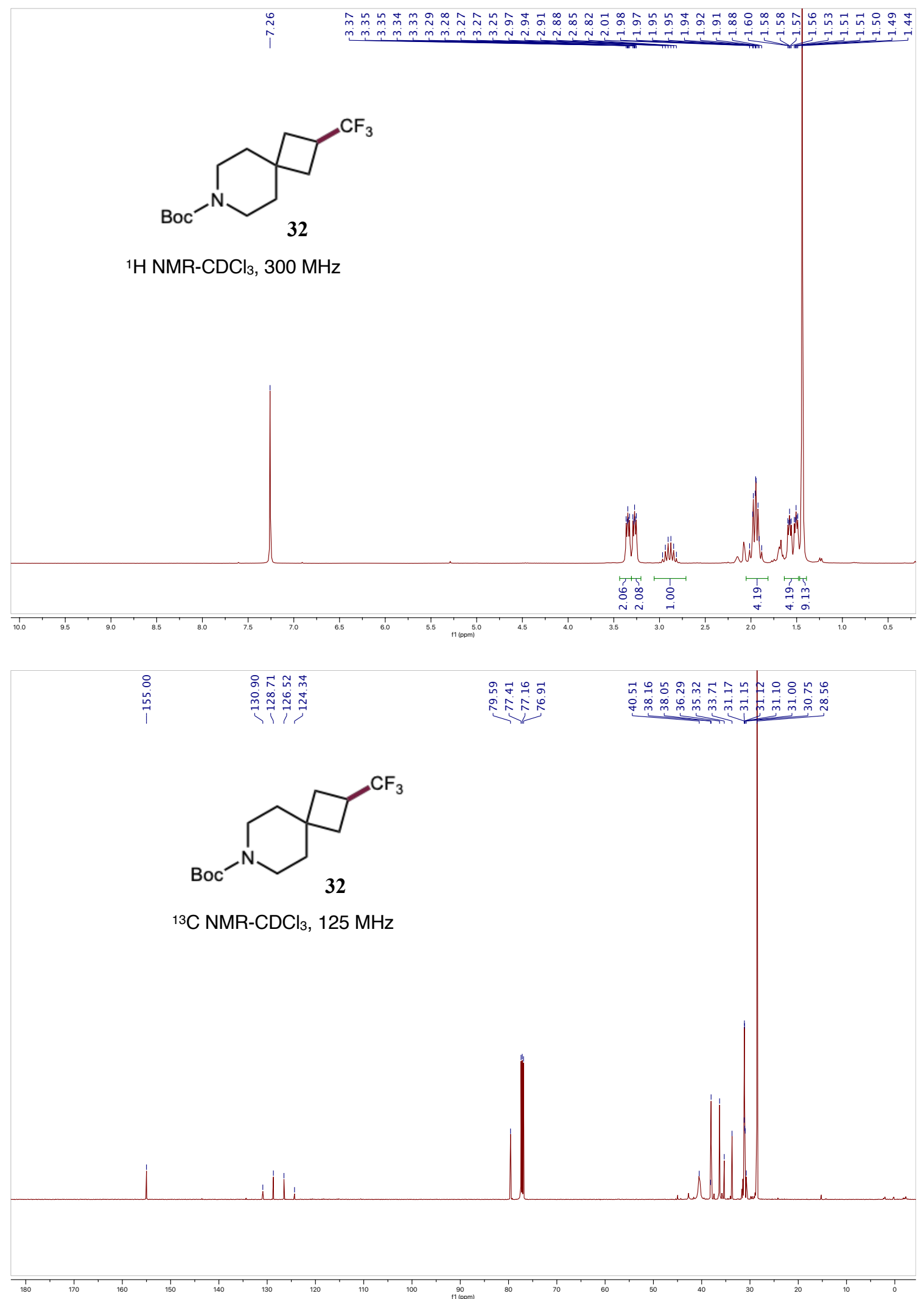


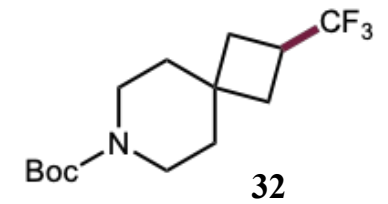

${ }^{19} \mathrm{~F} \mathrm{NMR-CDCl} 3,282 \mathrm{MHz}$

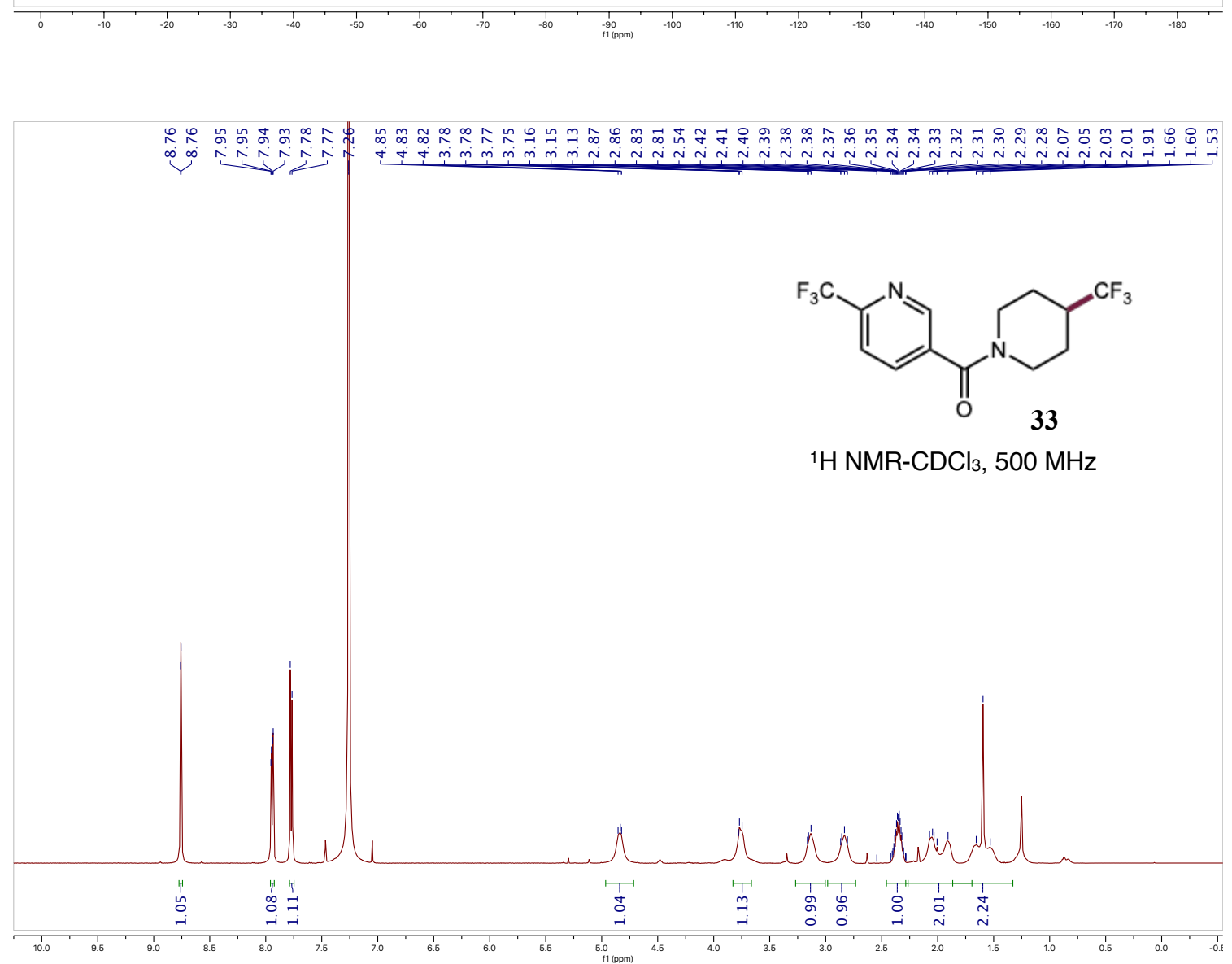



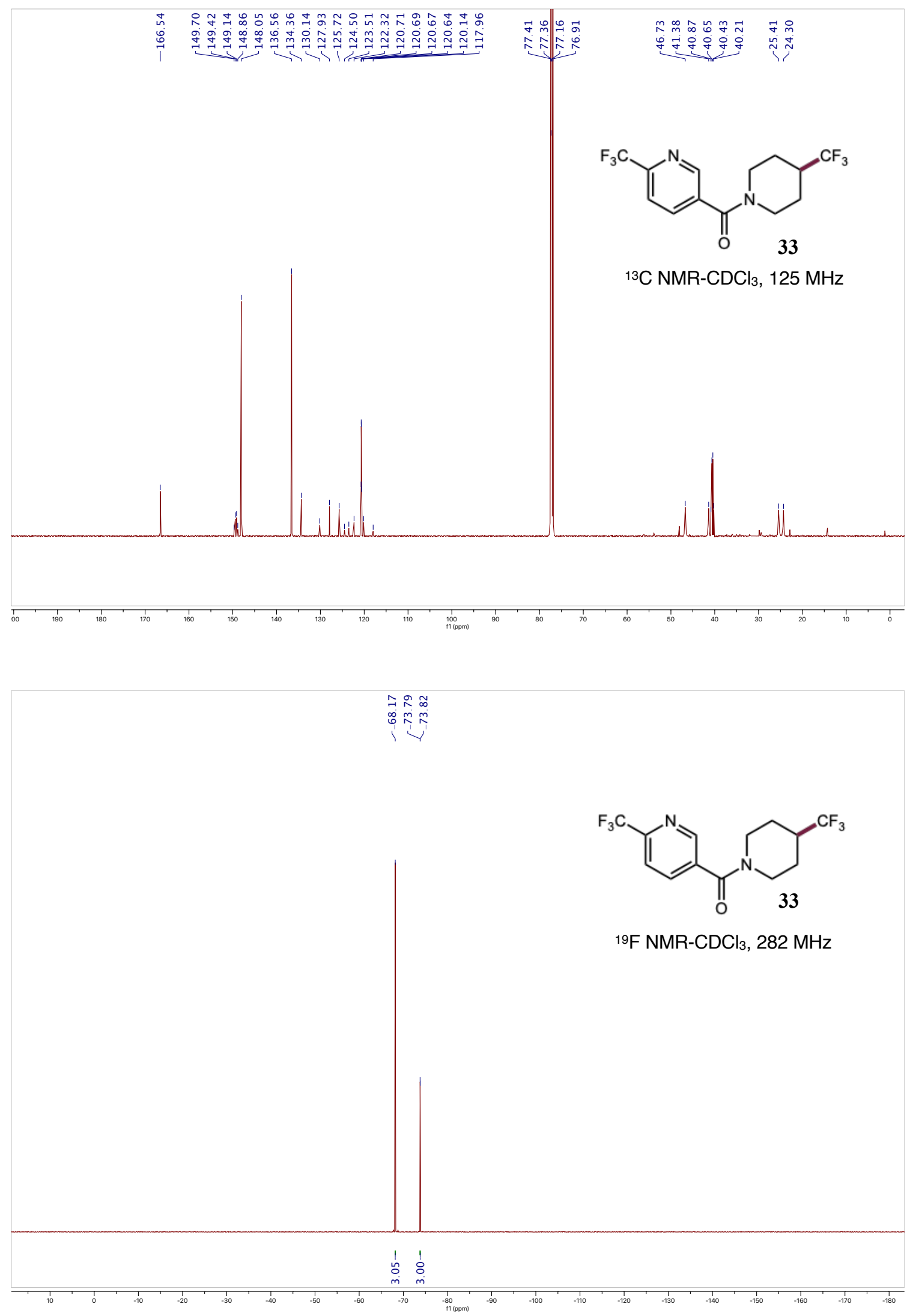


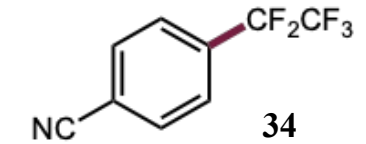

${ }^{1} \mathrm{H} \mathrm{NMR-CDCl}{ }_{3}, 400 \mathrm{MHz}$

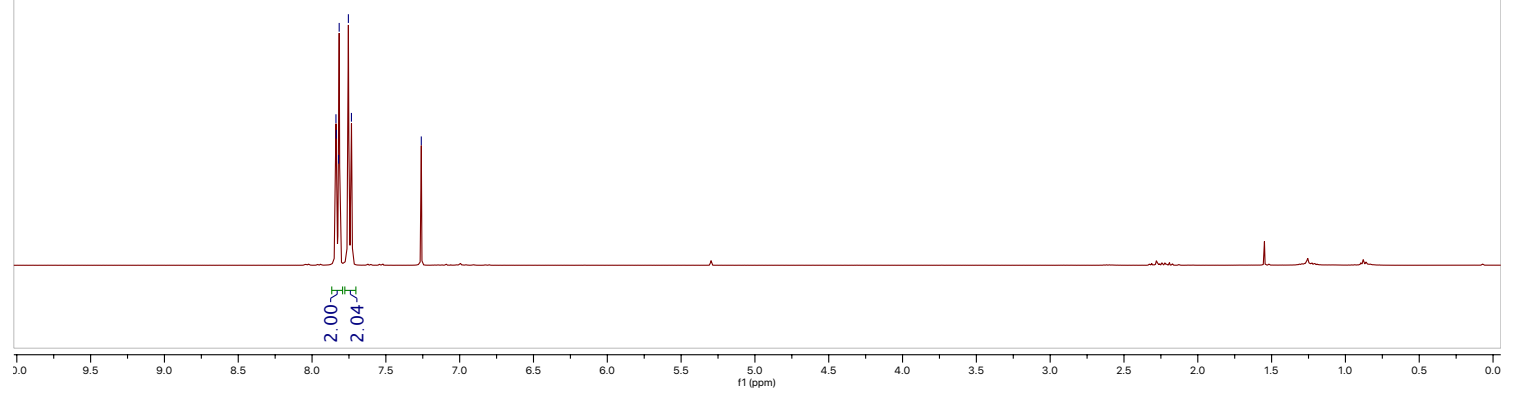

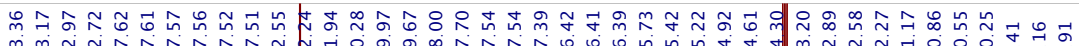

m.
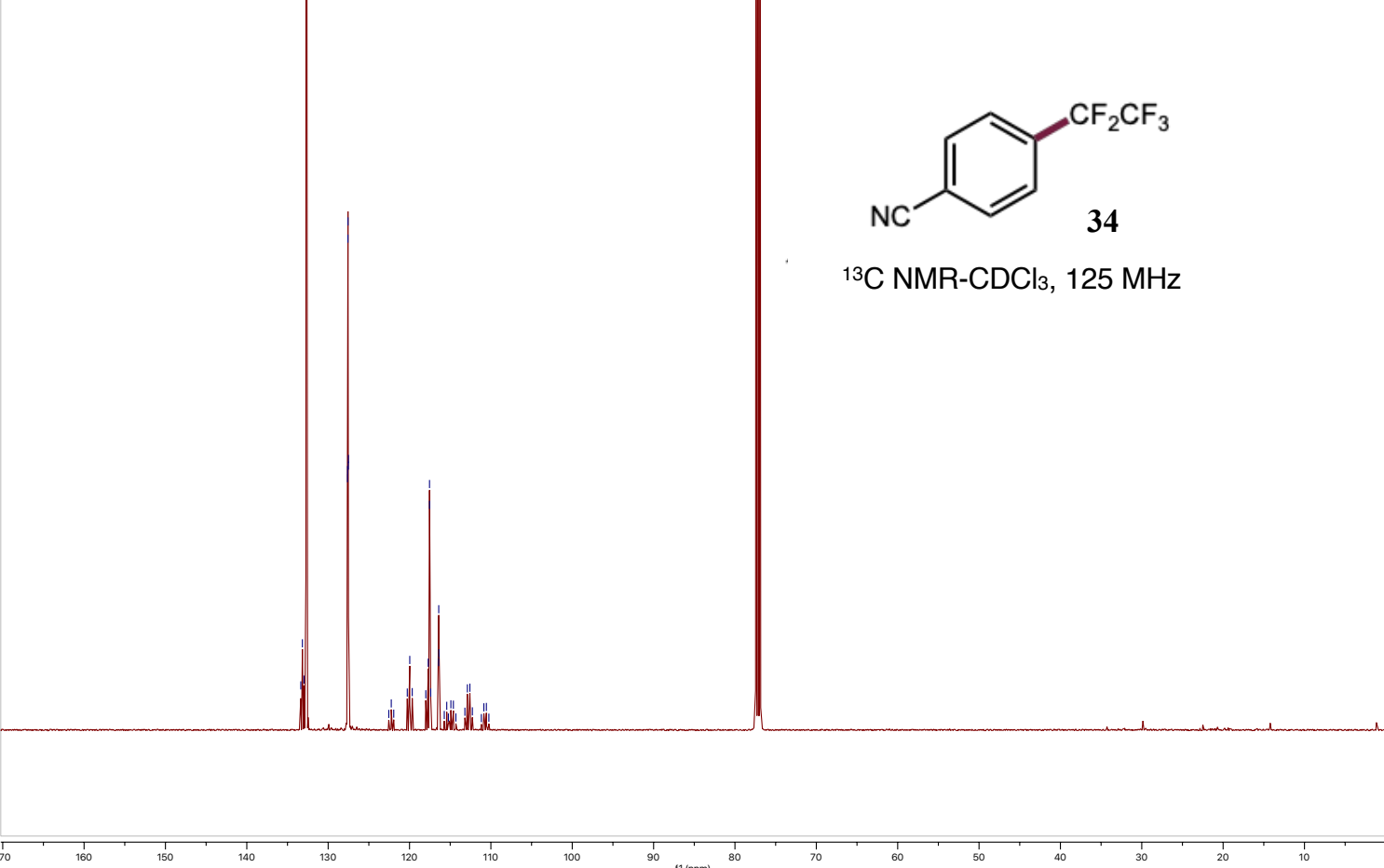


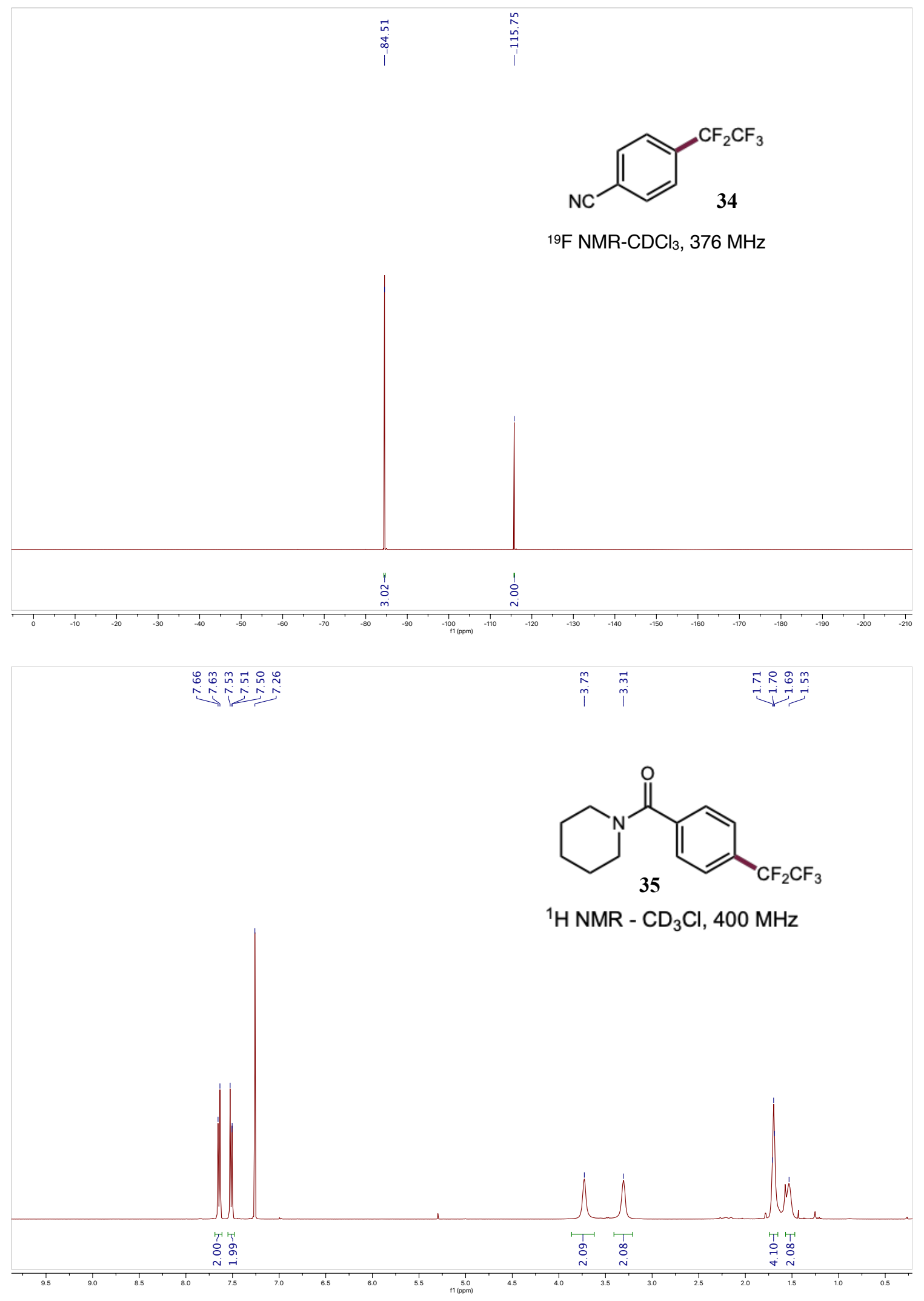



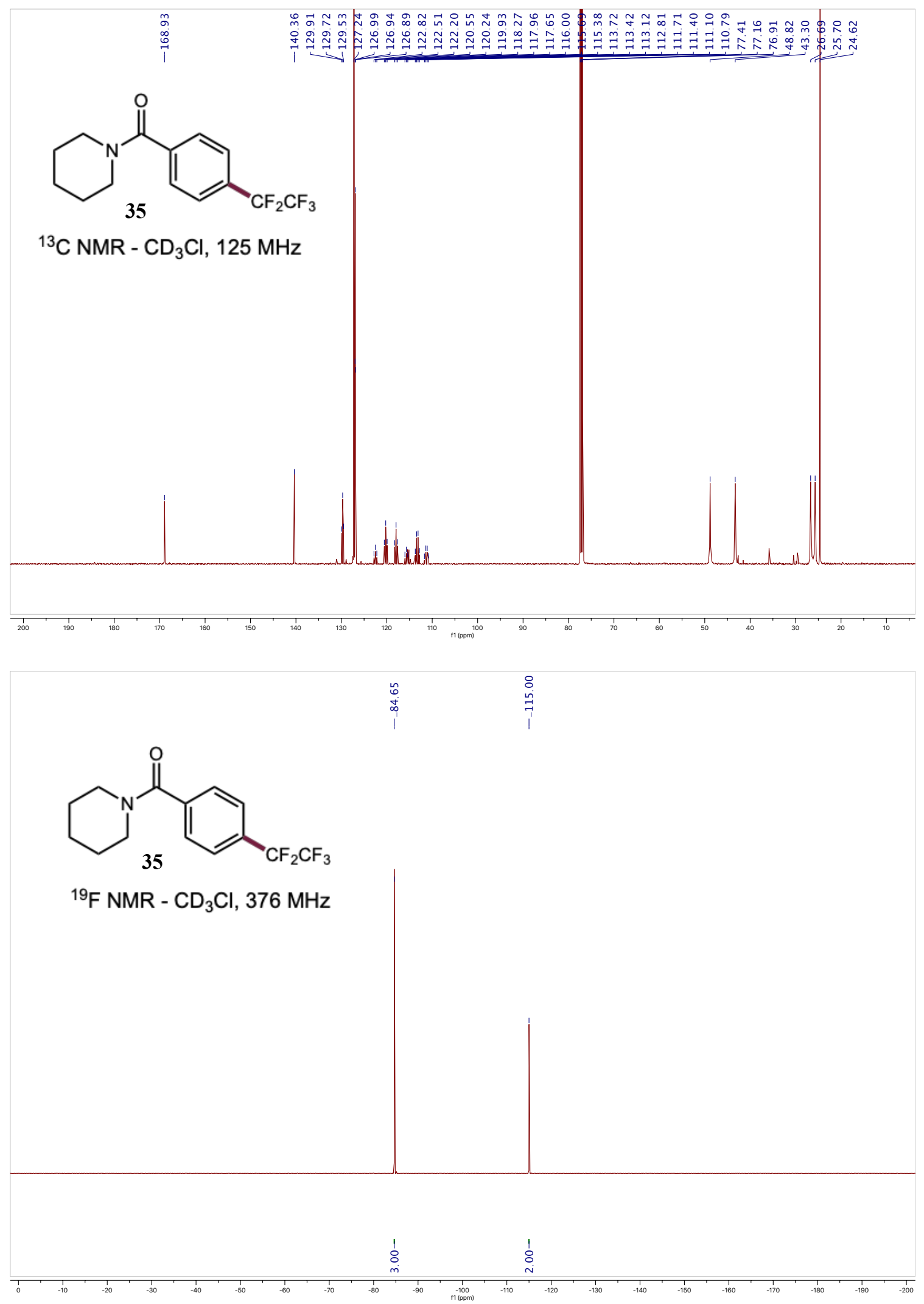


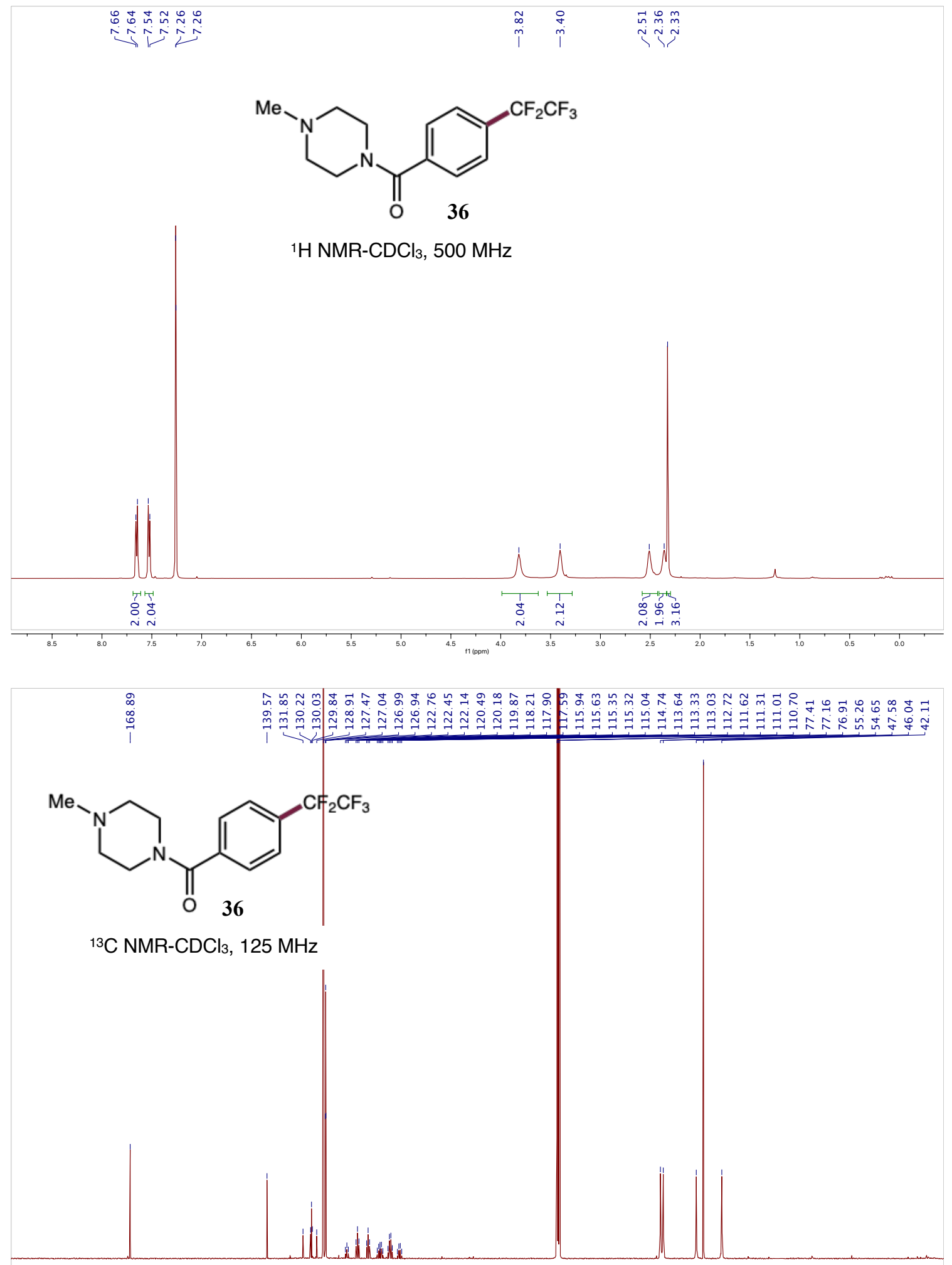




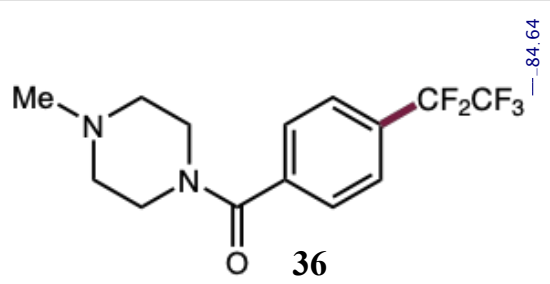

${ }^{19} \mathrm{~F} \mathrm{NMR}-\mathrm{CDCl}_{3}, 282 \mathrm{MHz}$

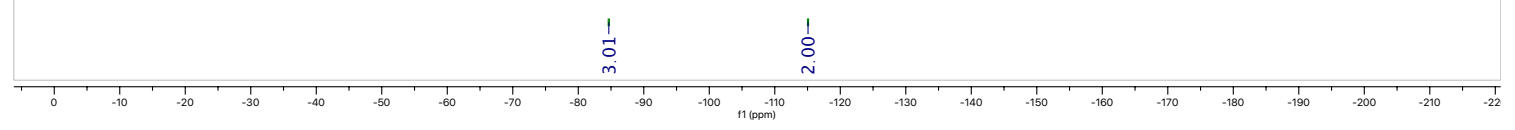

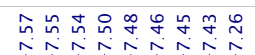

ninininisinas

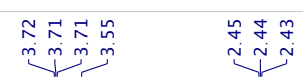<smiles>FC(F)(F)C(F)(F)c1cccc(CN2CCOCC2)c1</smiles>

${ }^{1} \mathrm{H} \mathrm{NMR}-\mathrm{CDCl}_{3}, 500 \mathrm{MHz}$

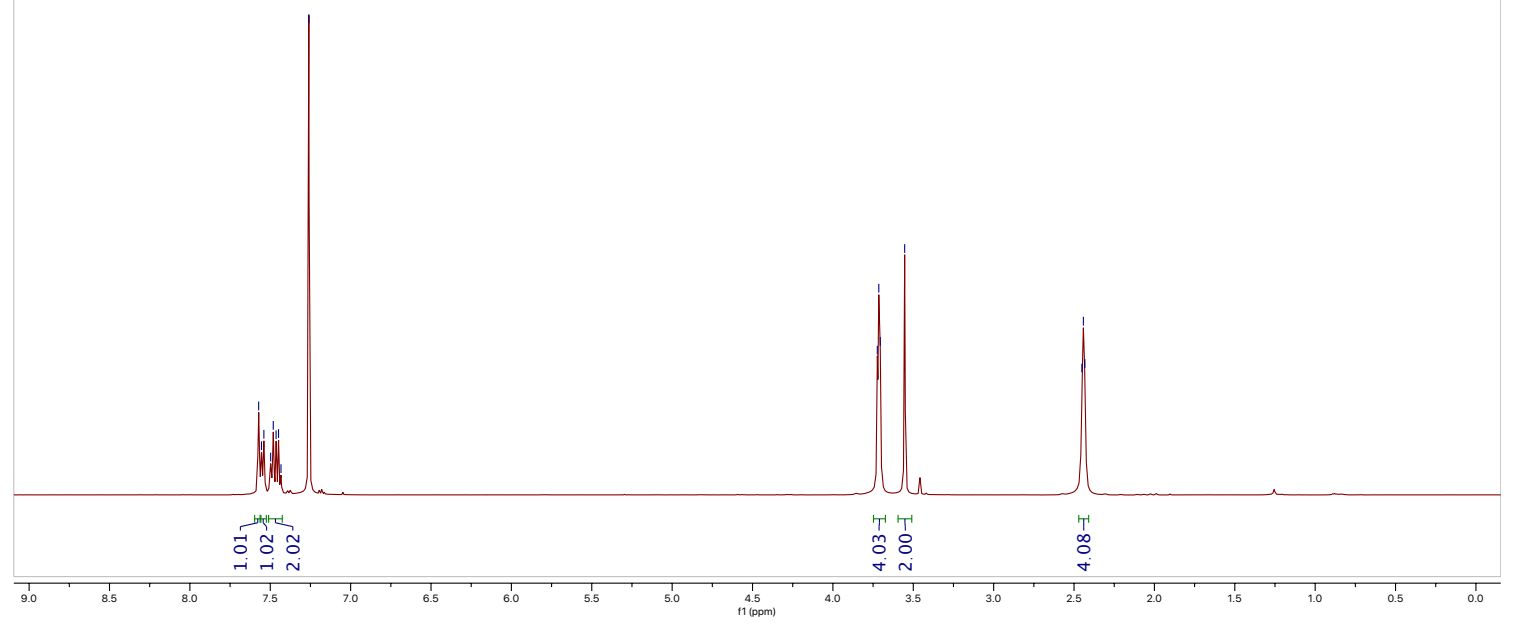



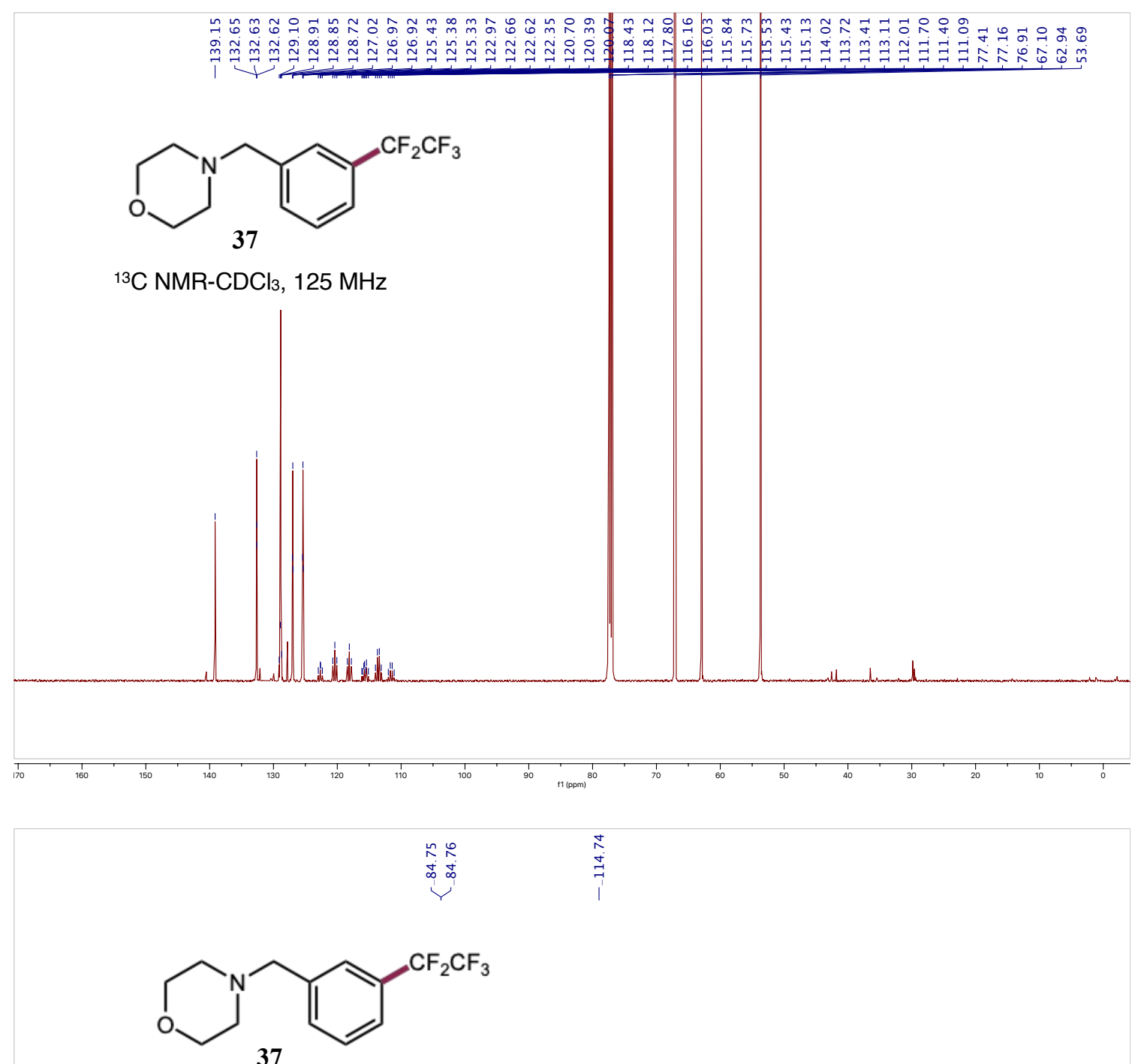

${ }_{19} \mathrm{~F} \mathrm{NMR-CDCl}, 282 \mathrm{MHz}$

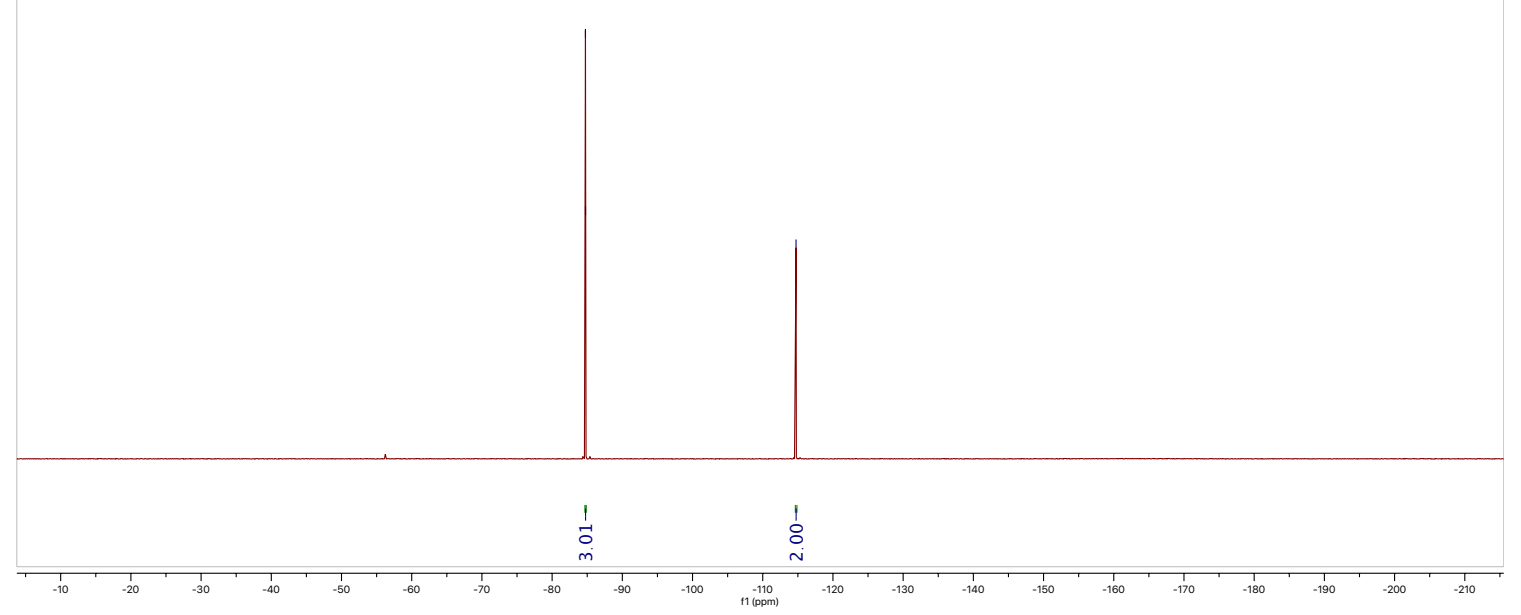




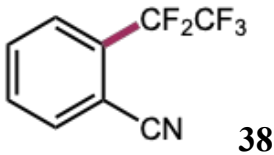

${ }^{1} \mathrm{H}$ NMR-CDCl $3,400 \mathrm{MHz}$

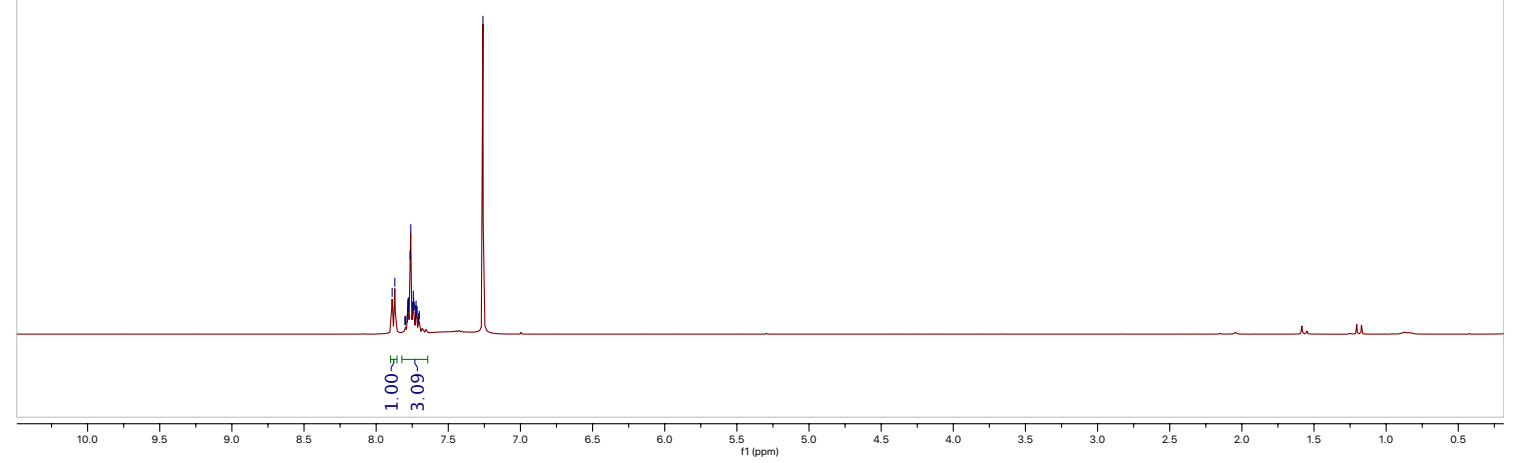

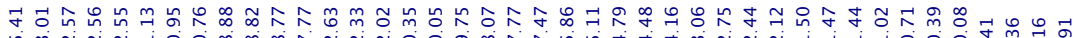

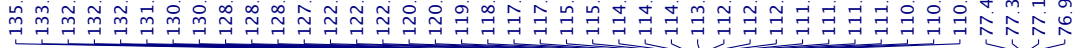

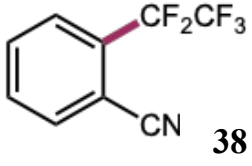

${ }^{13} \mathrm{C}$ NMR-CDCl $3,125 \mathrm{MHz}$

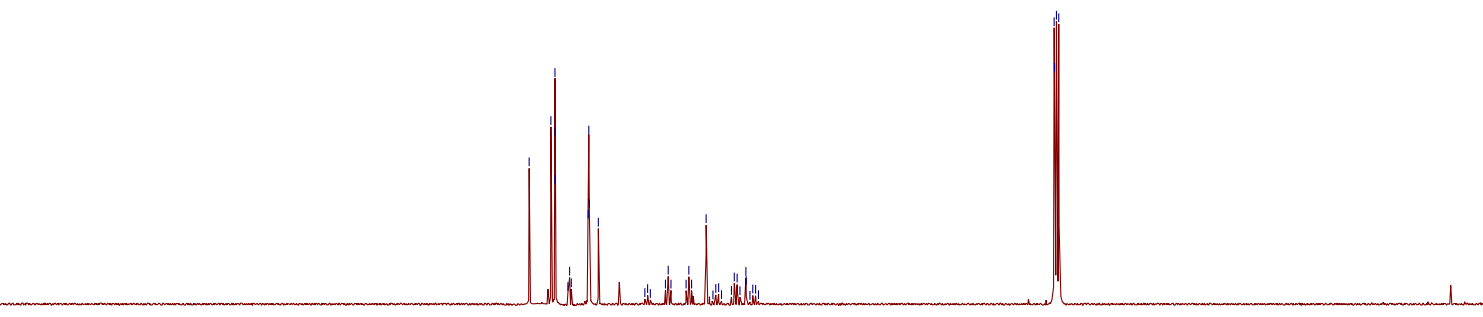



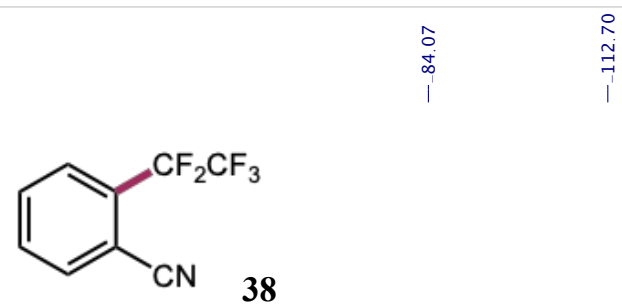

${ }^{19} \mathrm{~F} \mathrm{NMR-CDCl} 3,376 \mathrm{MHz}$
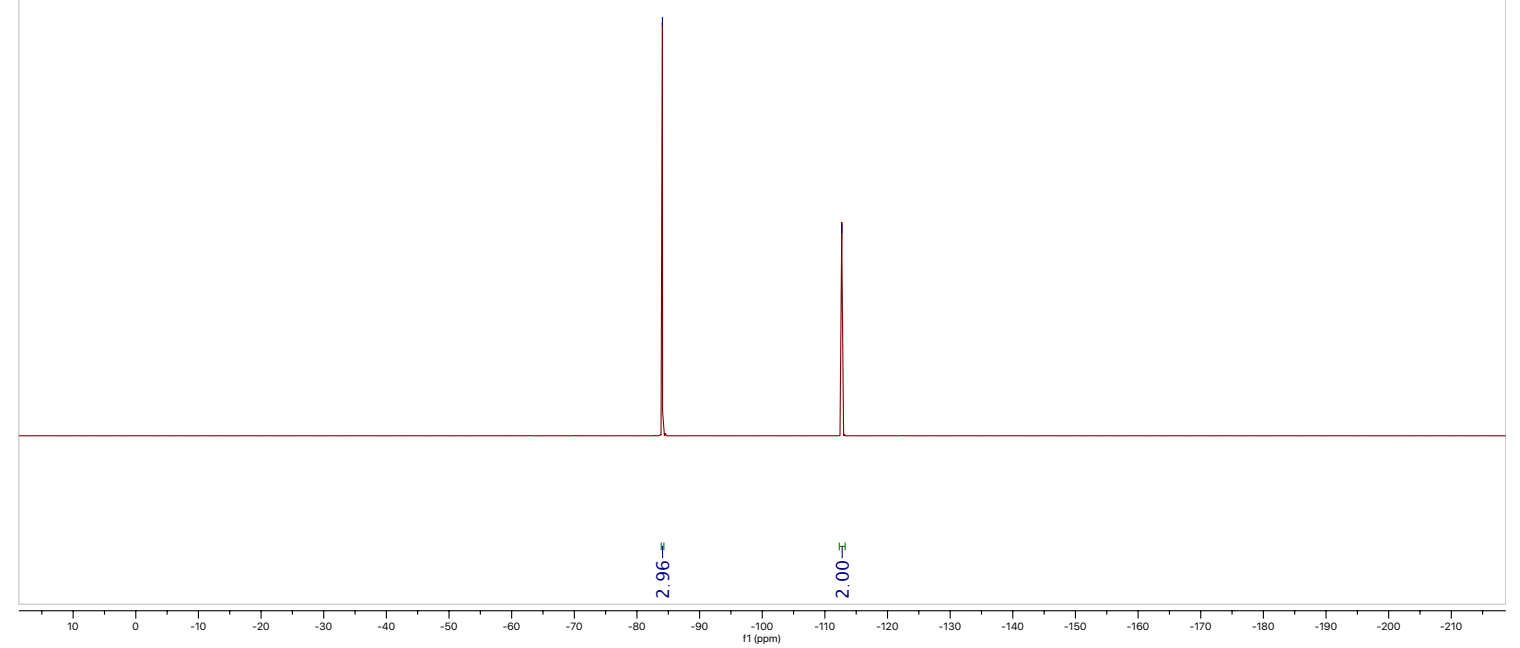

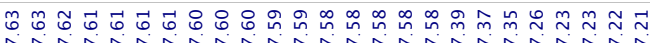

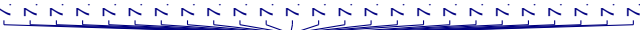

$\stackrel{\vec{m}}{i}$<smiles>CC(=O)Oc1ccccc1C(F)(F)C(F)(F)F</smiles>

${ }^{1} \mathrm{H} \mathrm{NMR-CDCl} 3,500 \mathrm{MHz}$

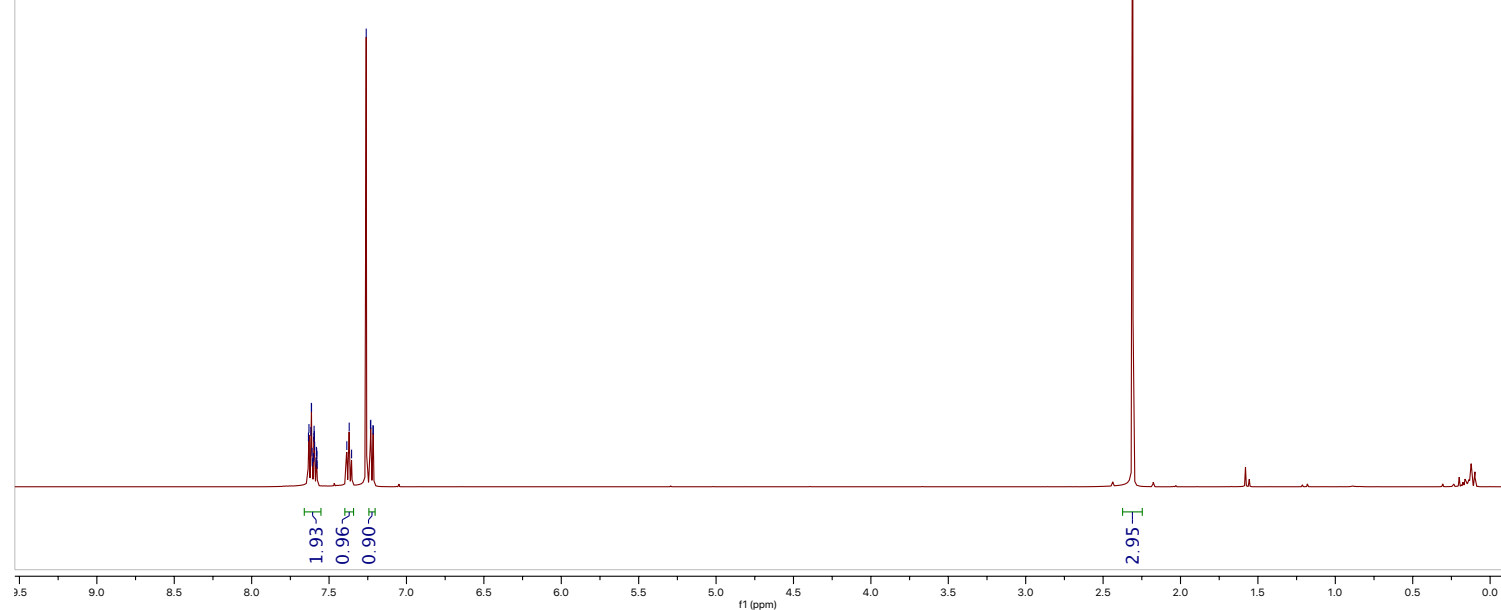



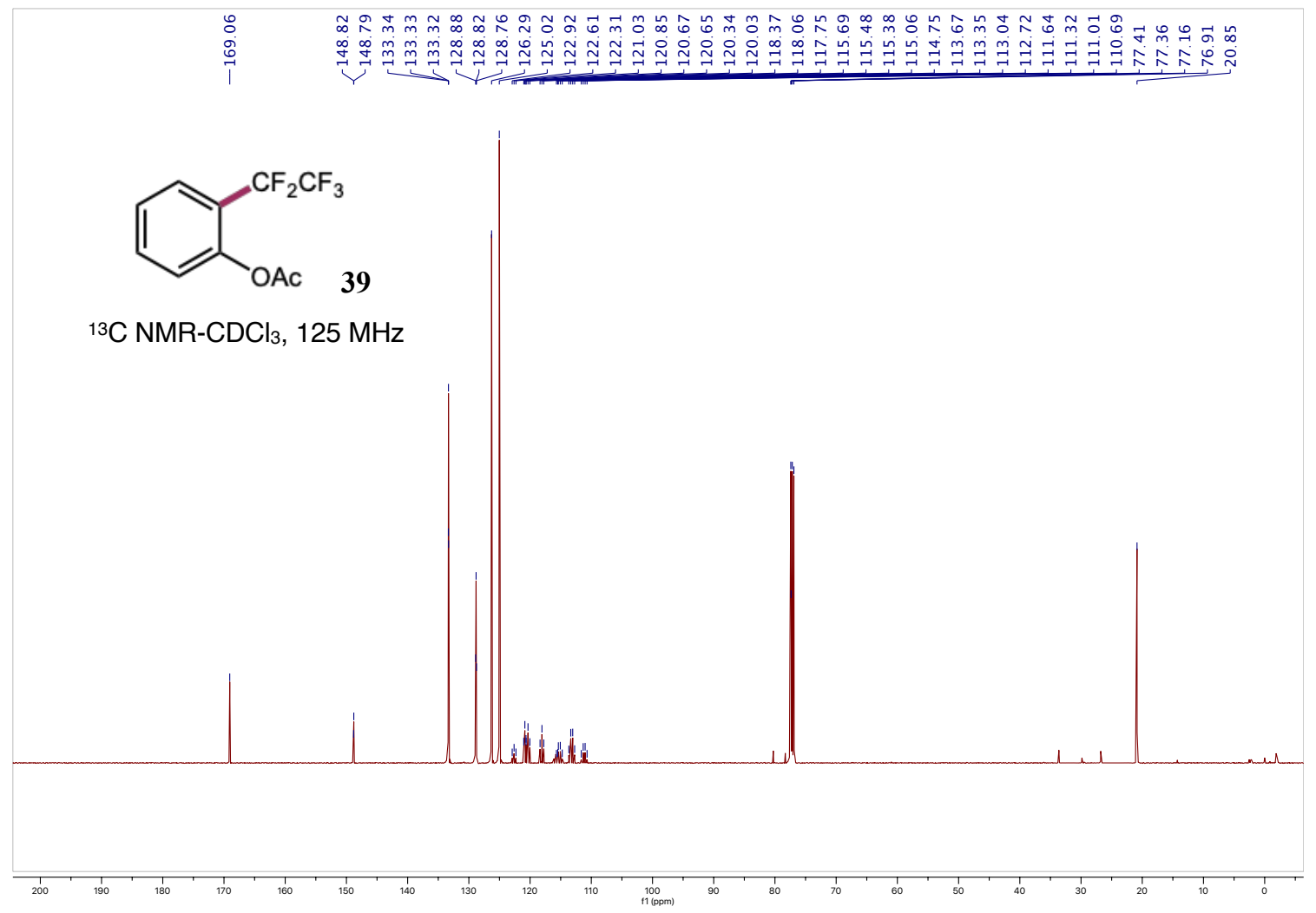

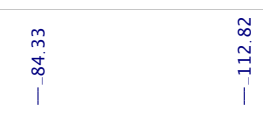

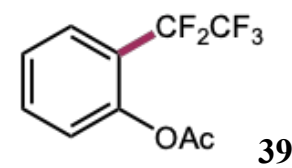

${ }_{19}^{19} \mathrm{NMR}-\mathrm{CDCl}_{3}, 376 \mathrm{MHz}$

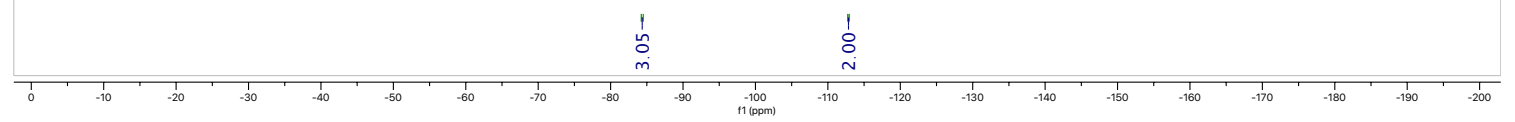



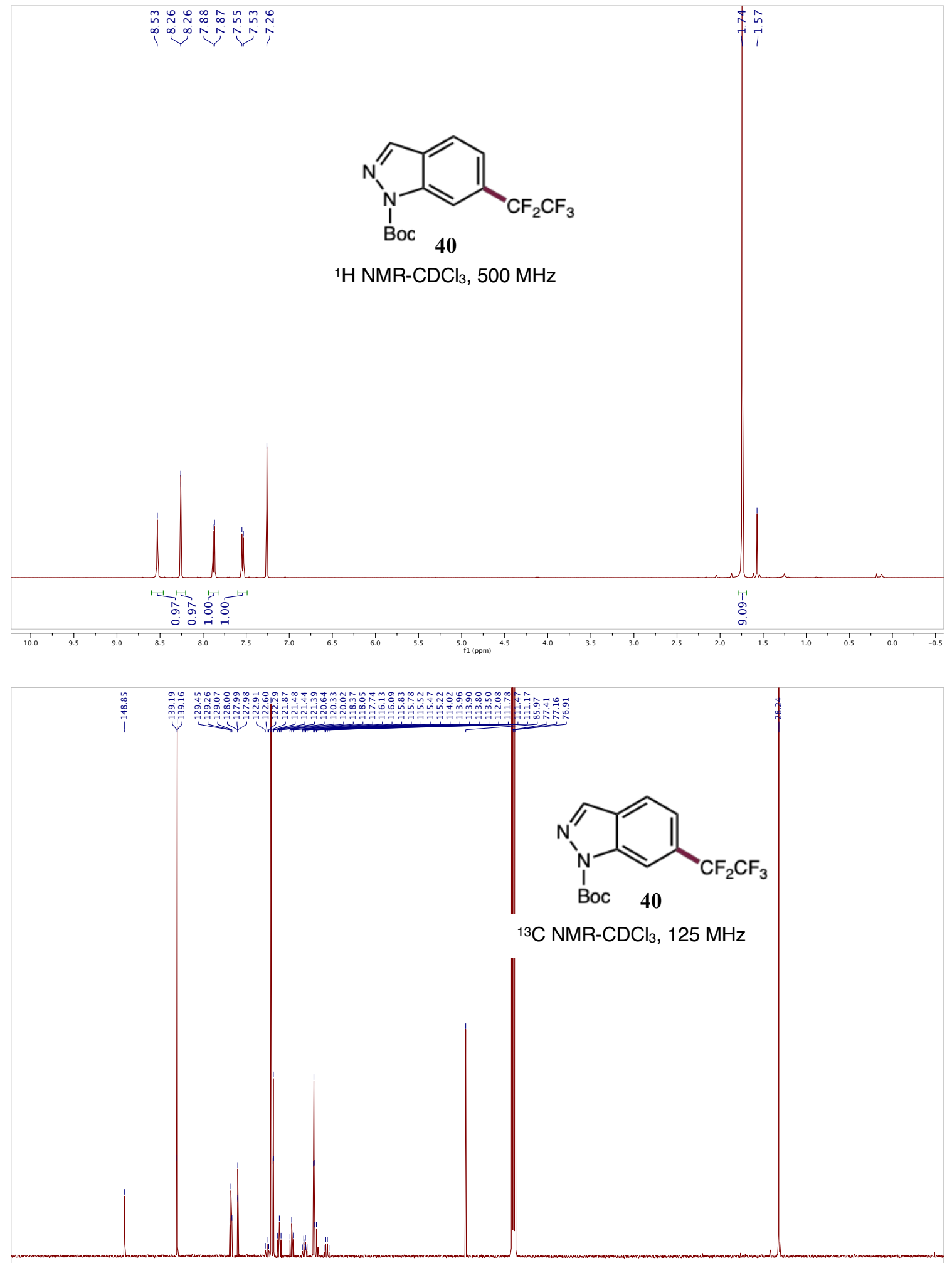


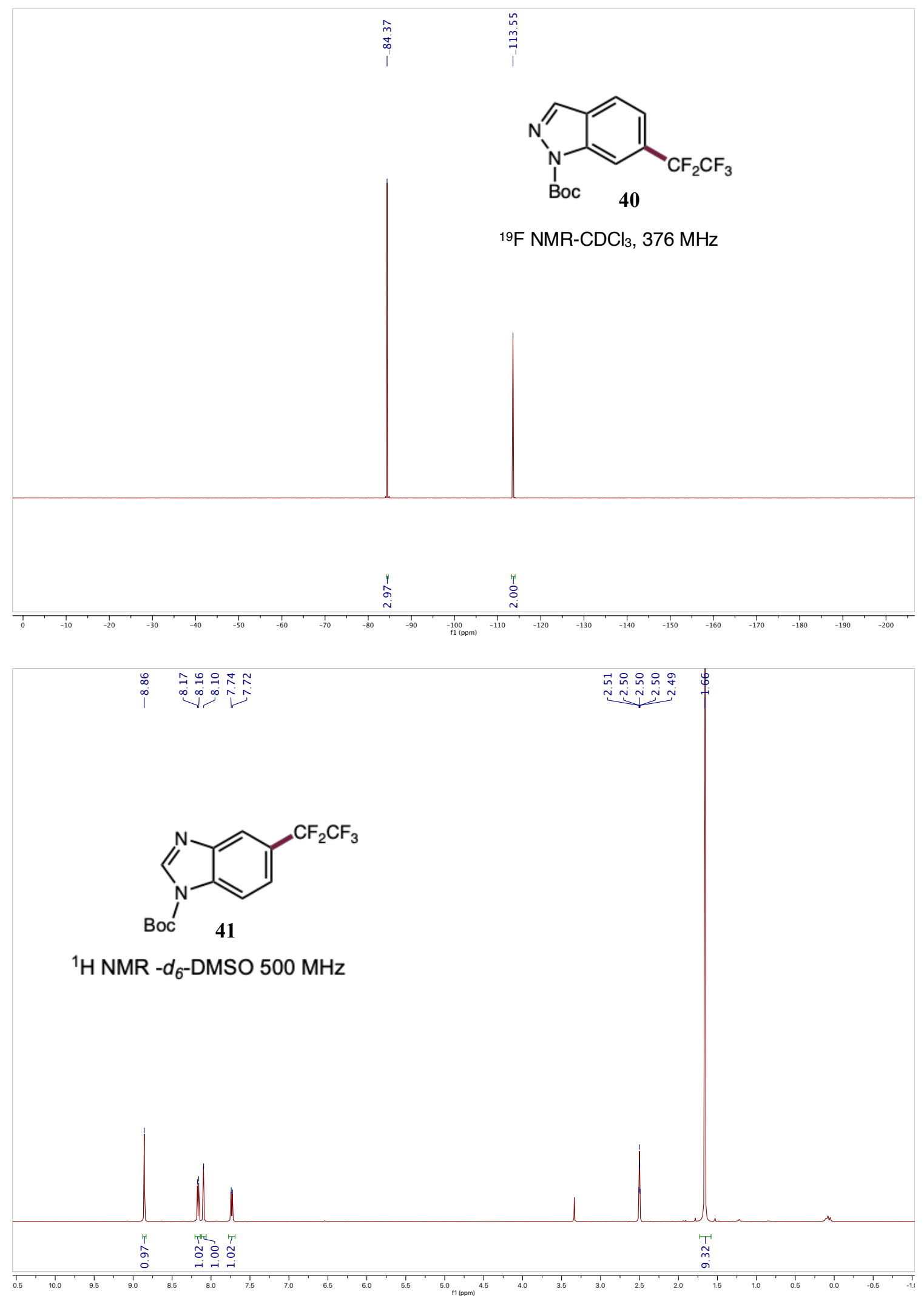



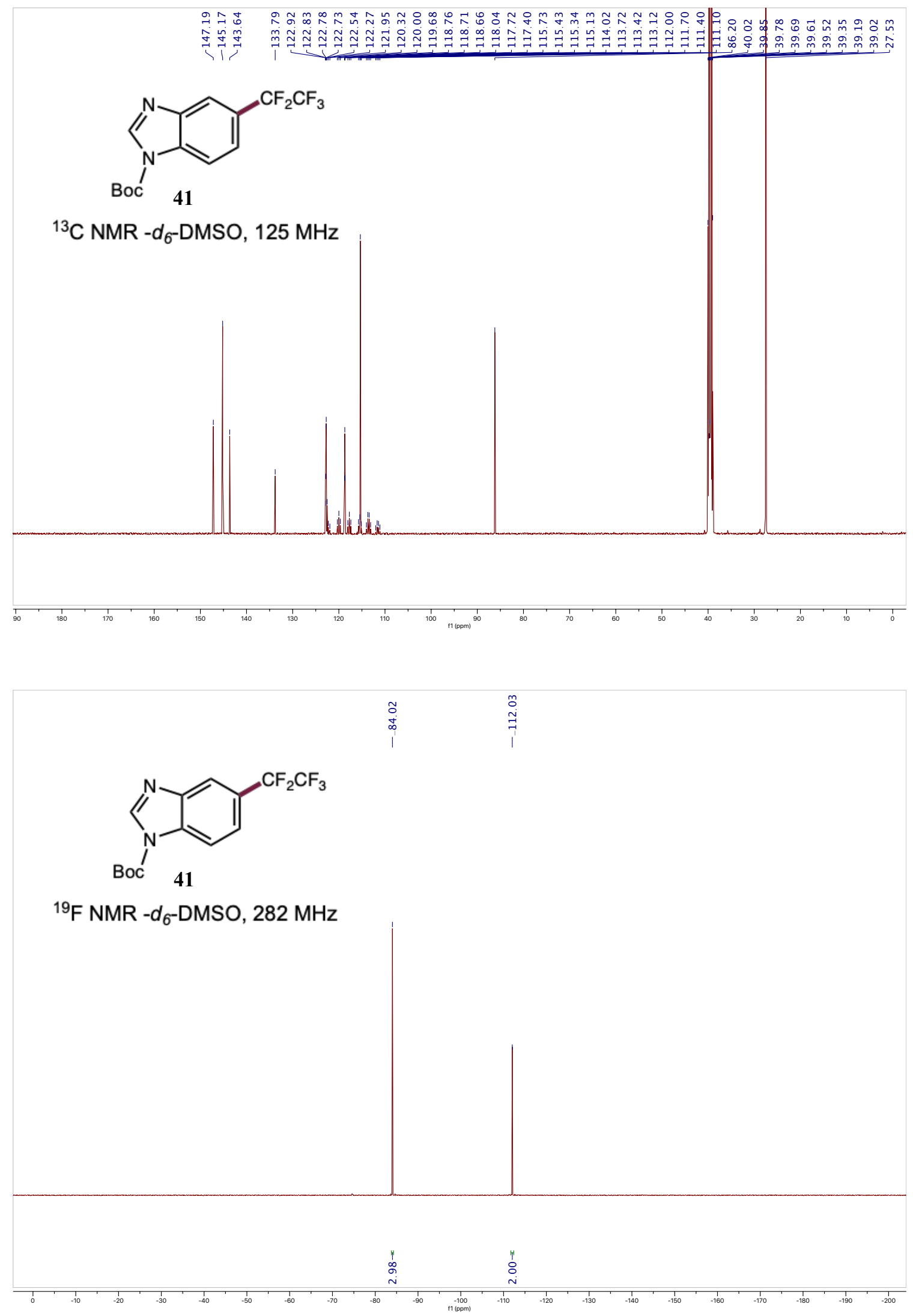

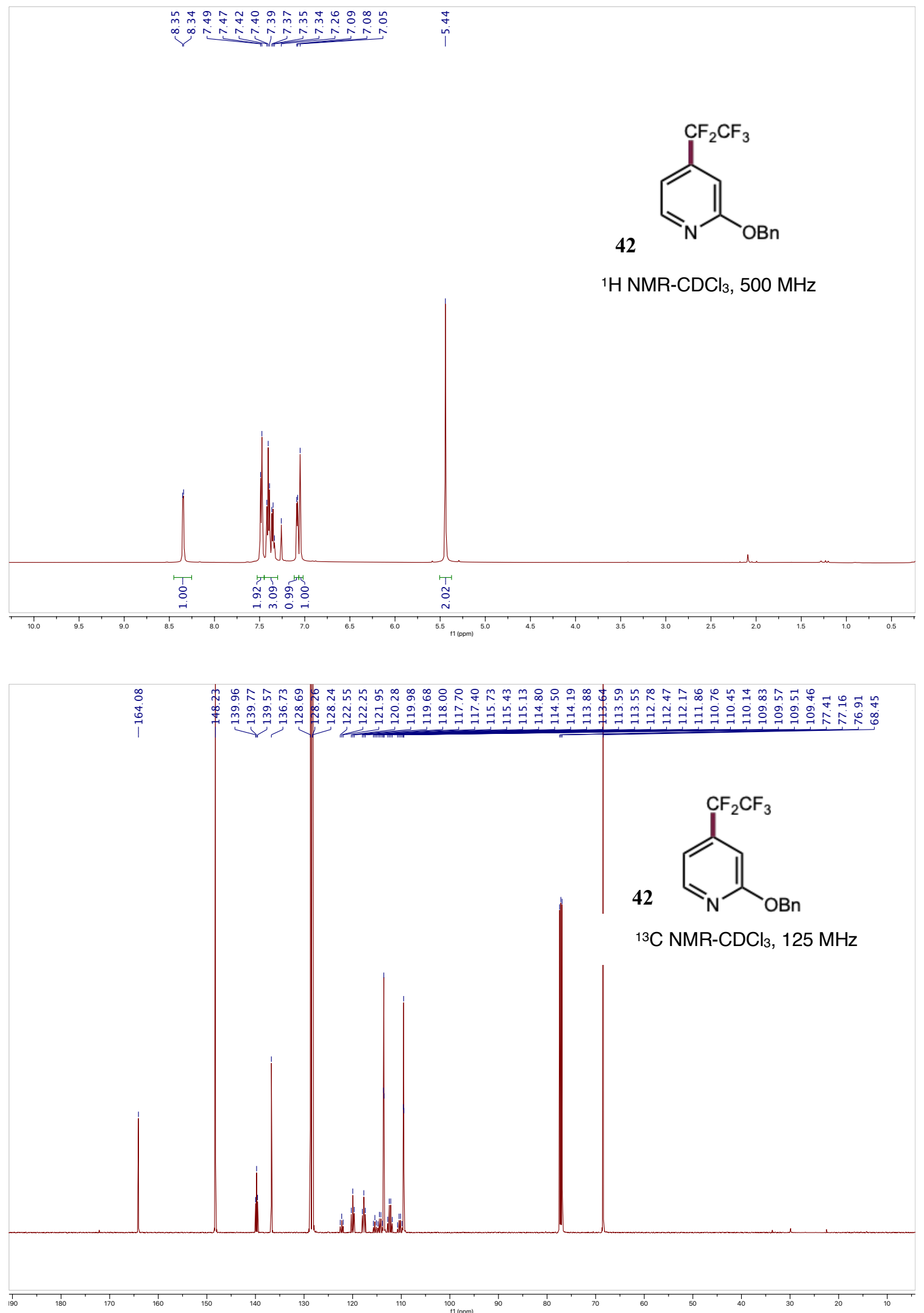


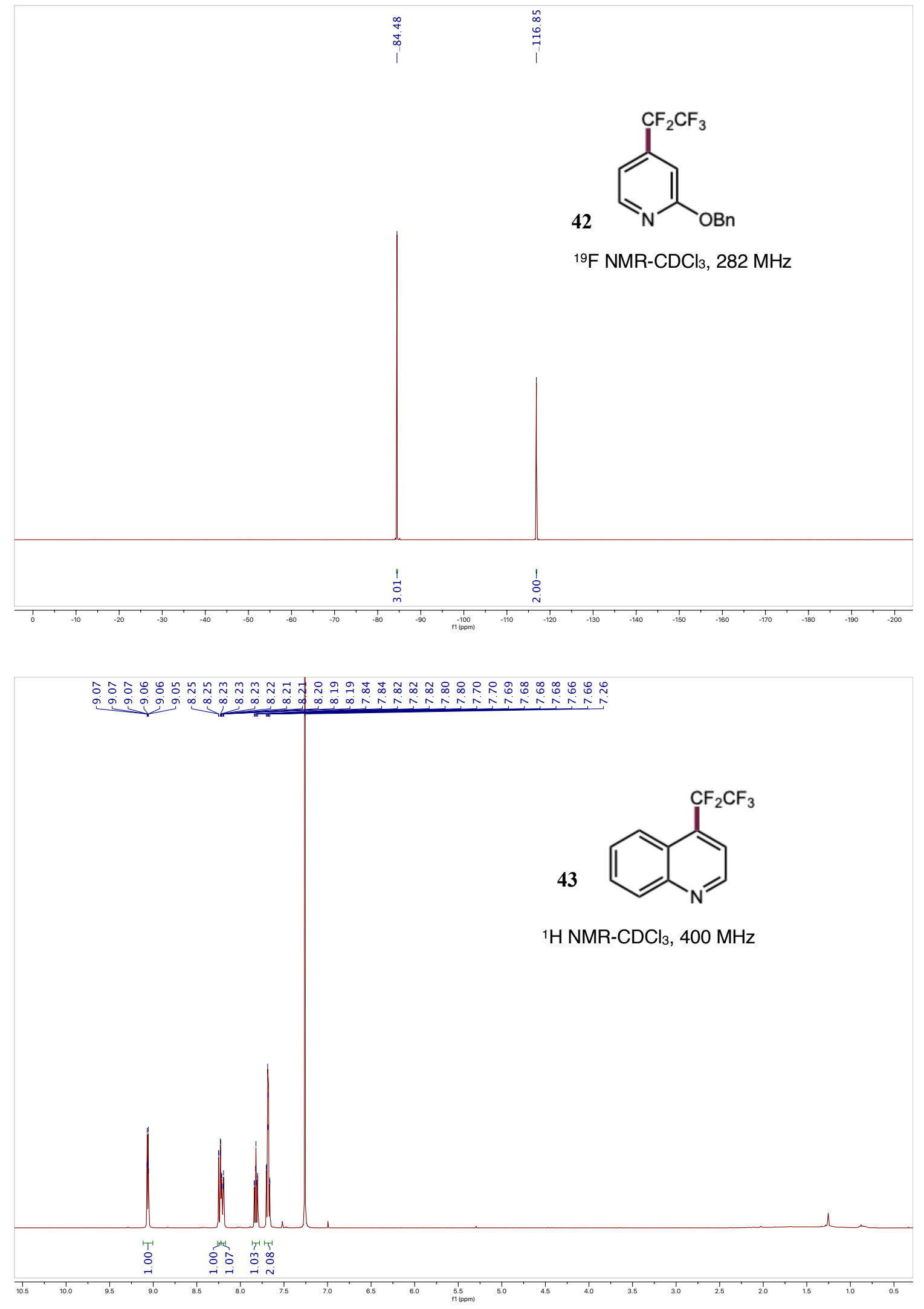



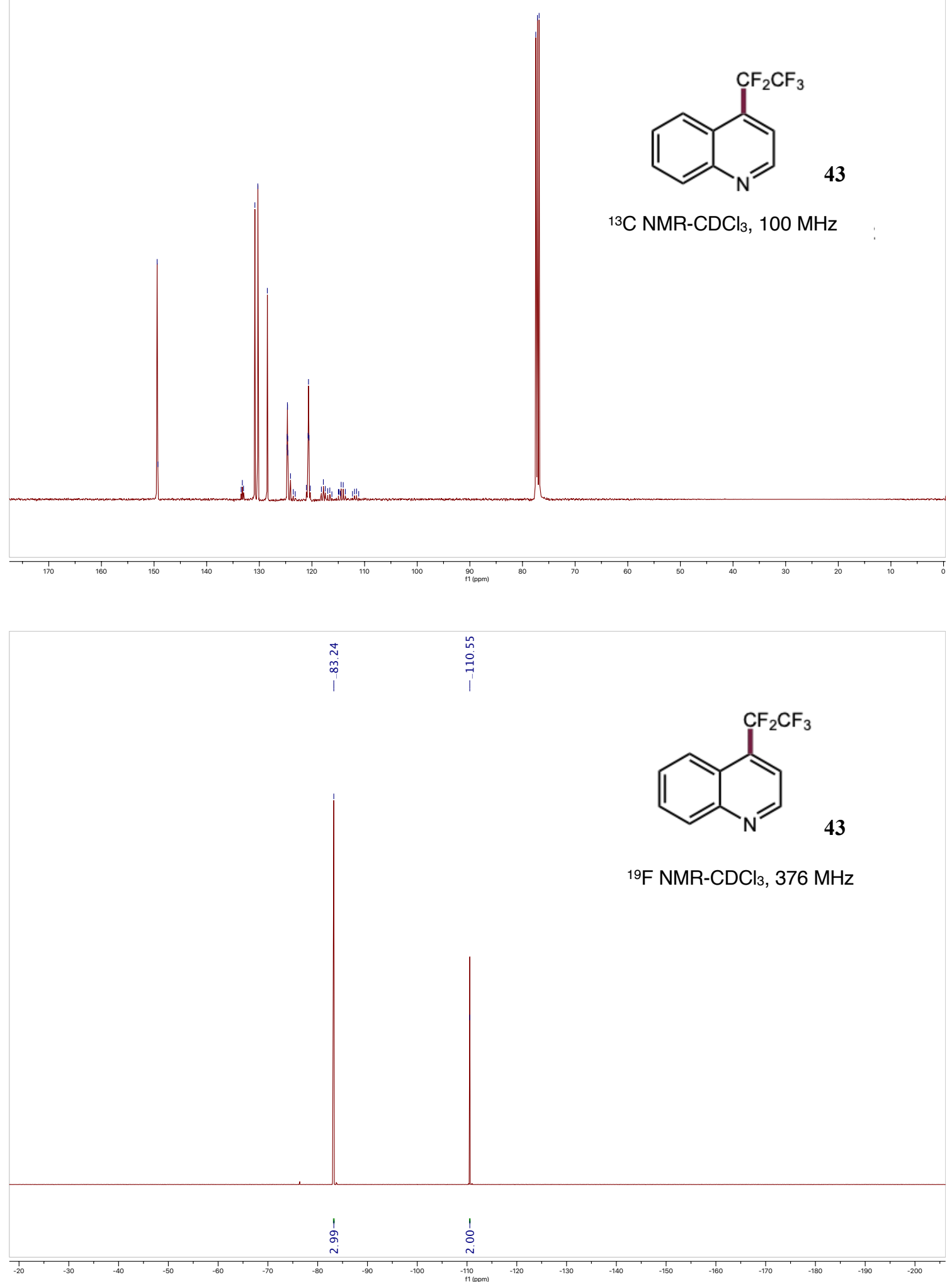


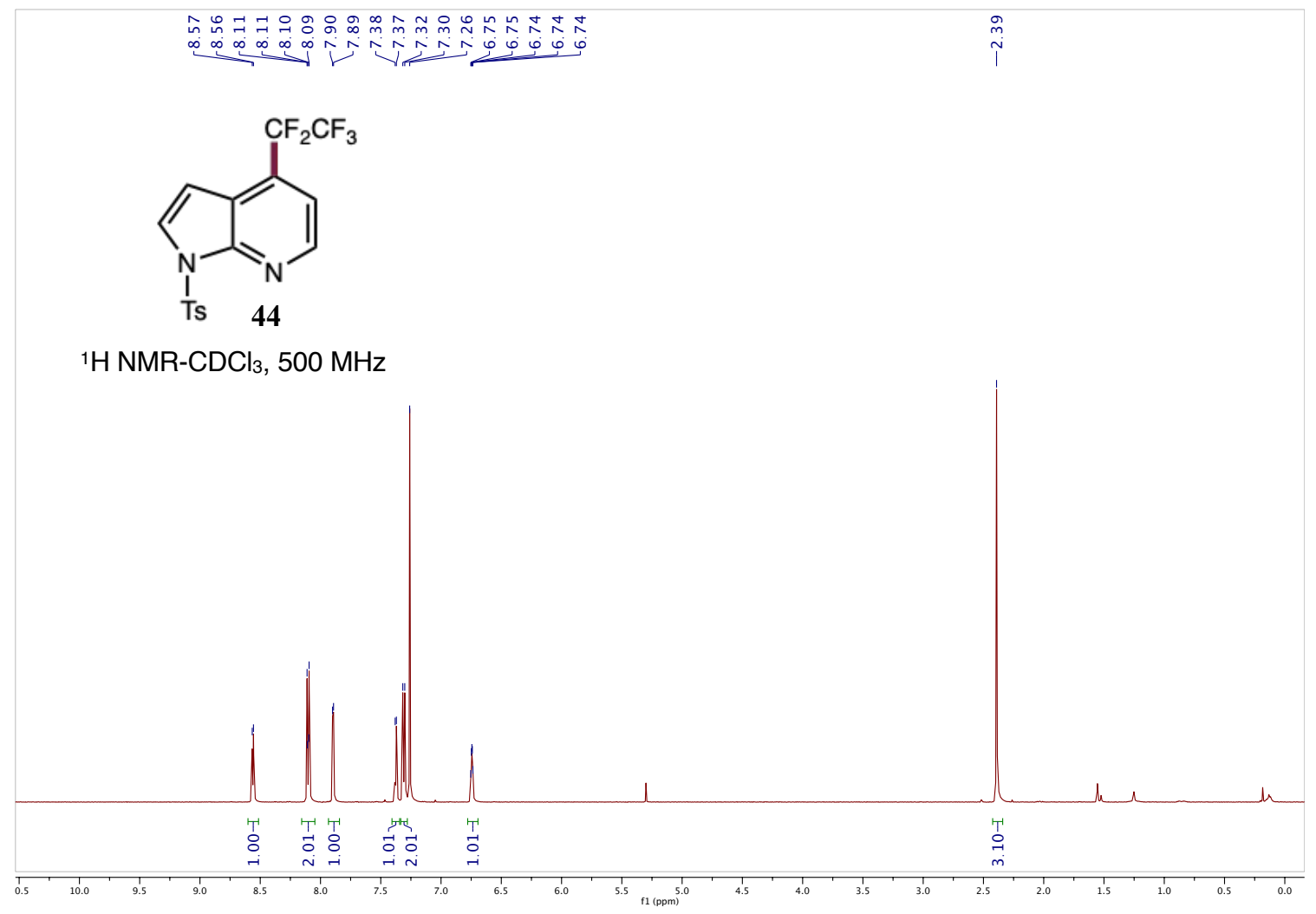

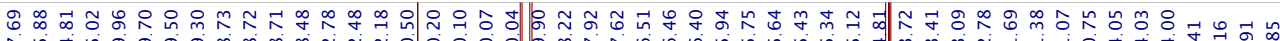

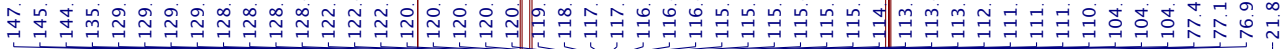
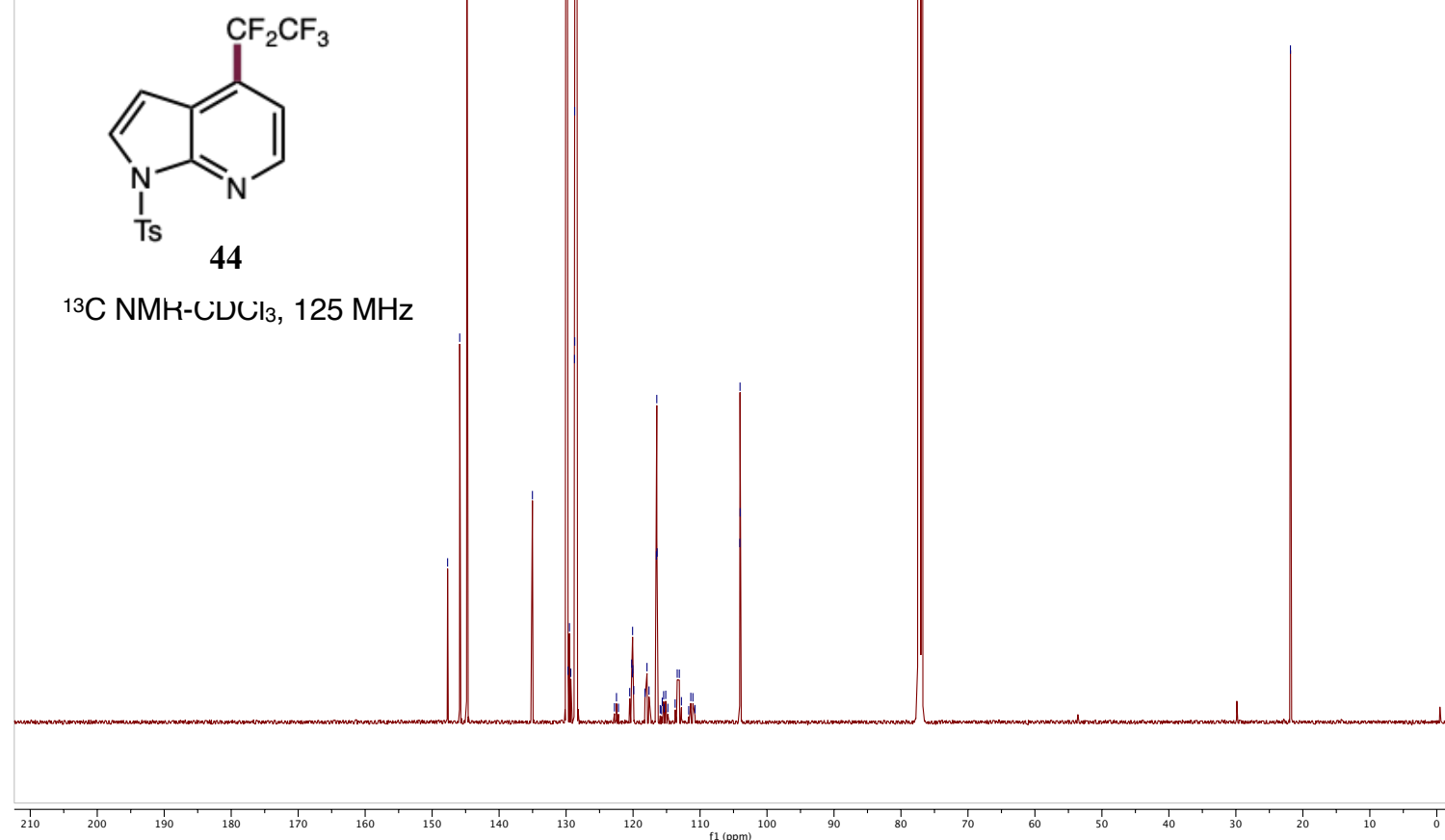


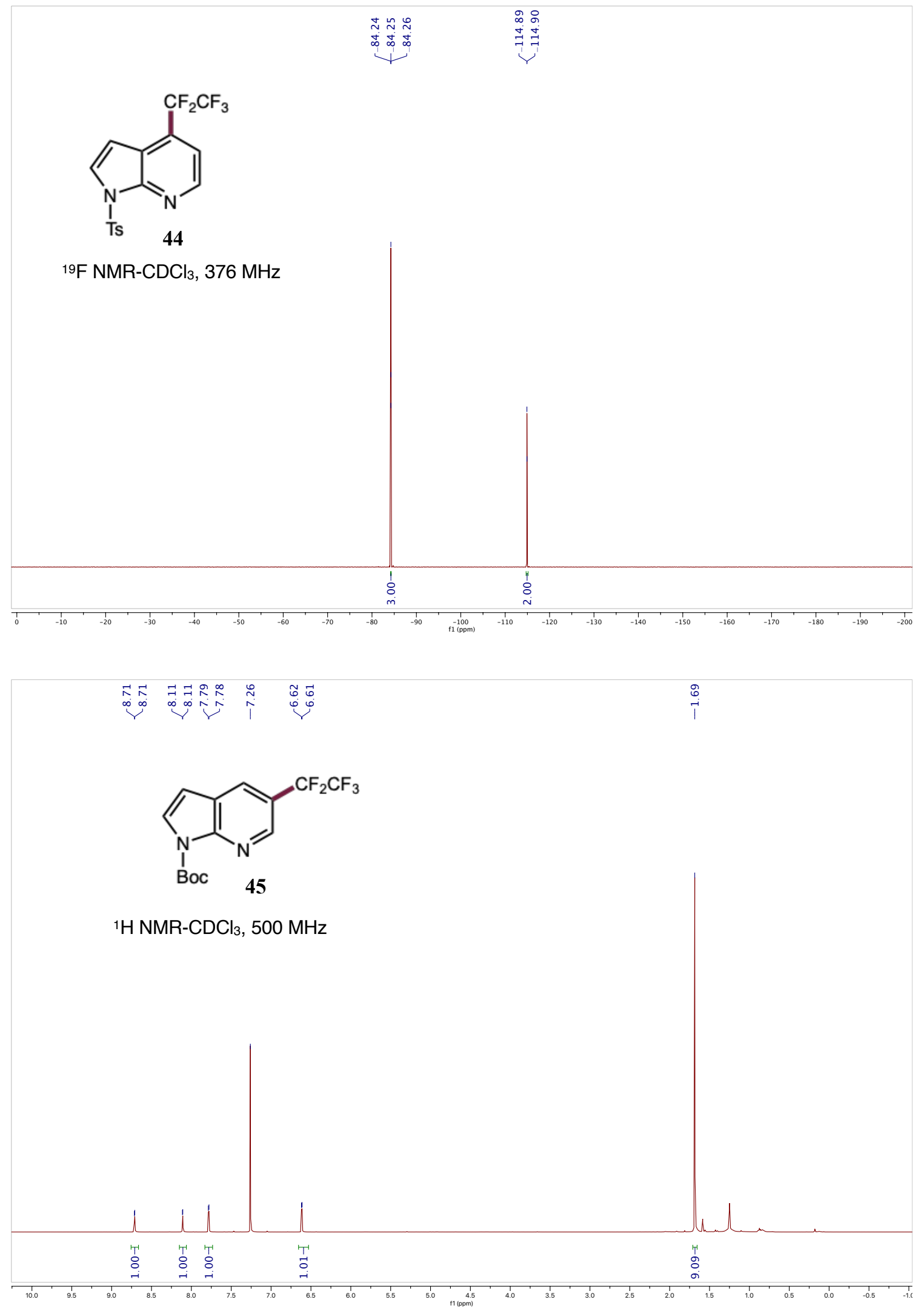



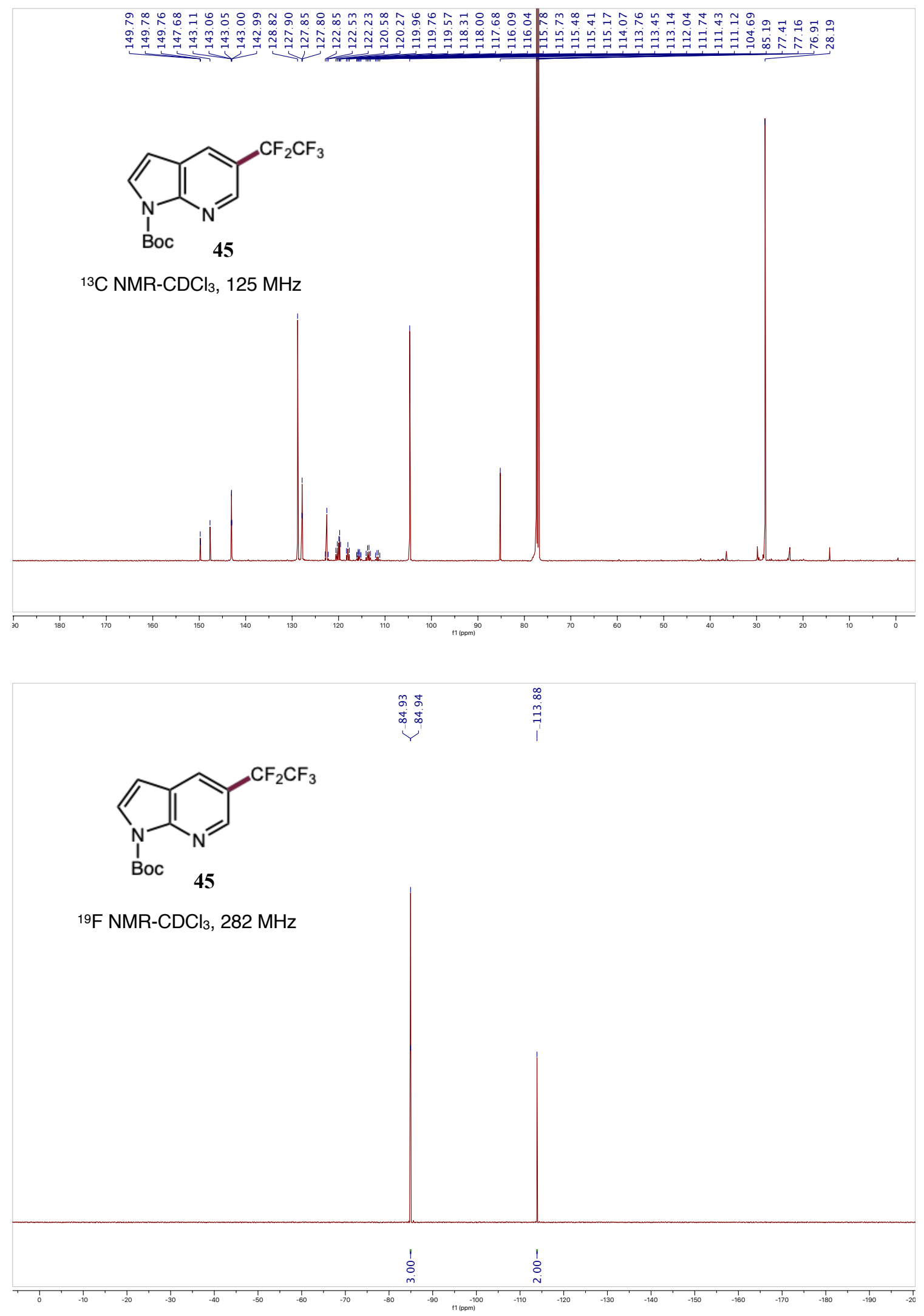

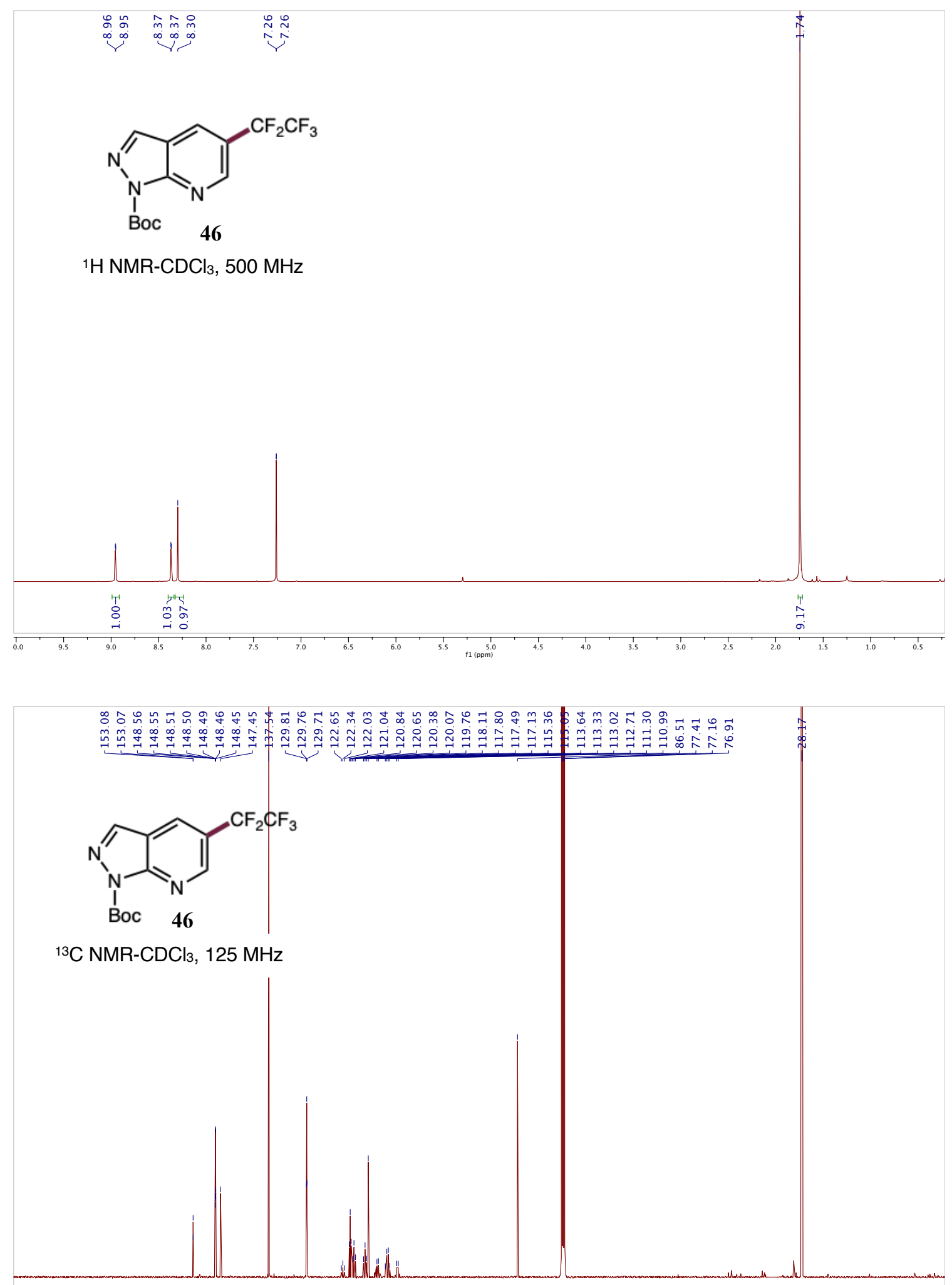


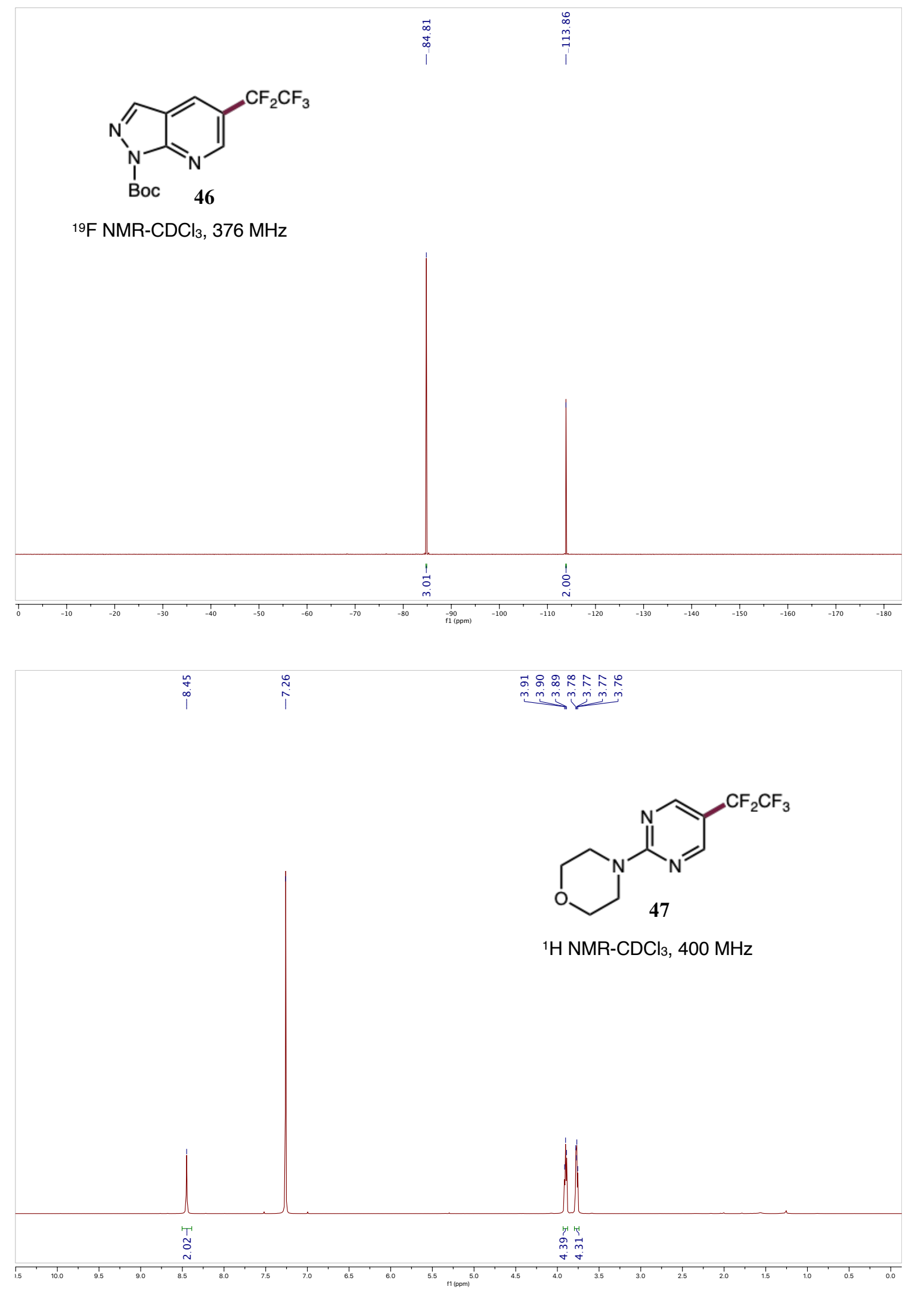



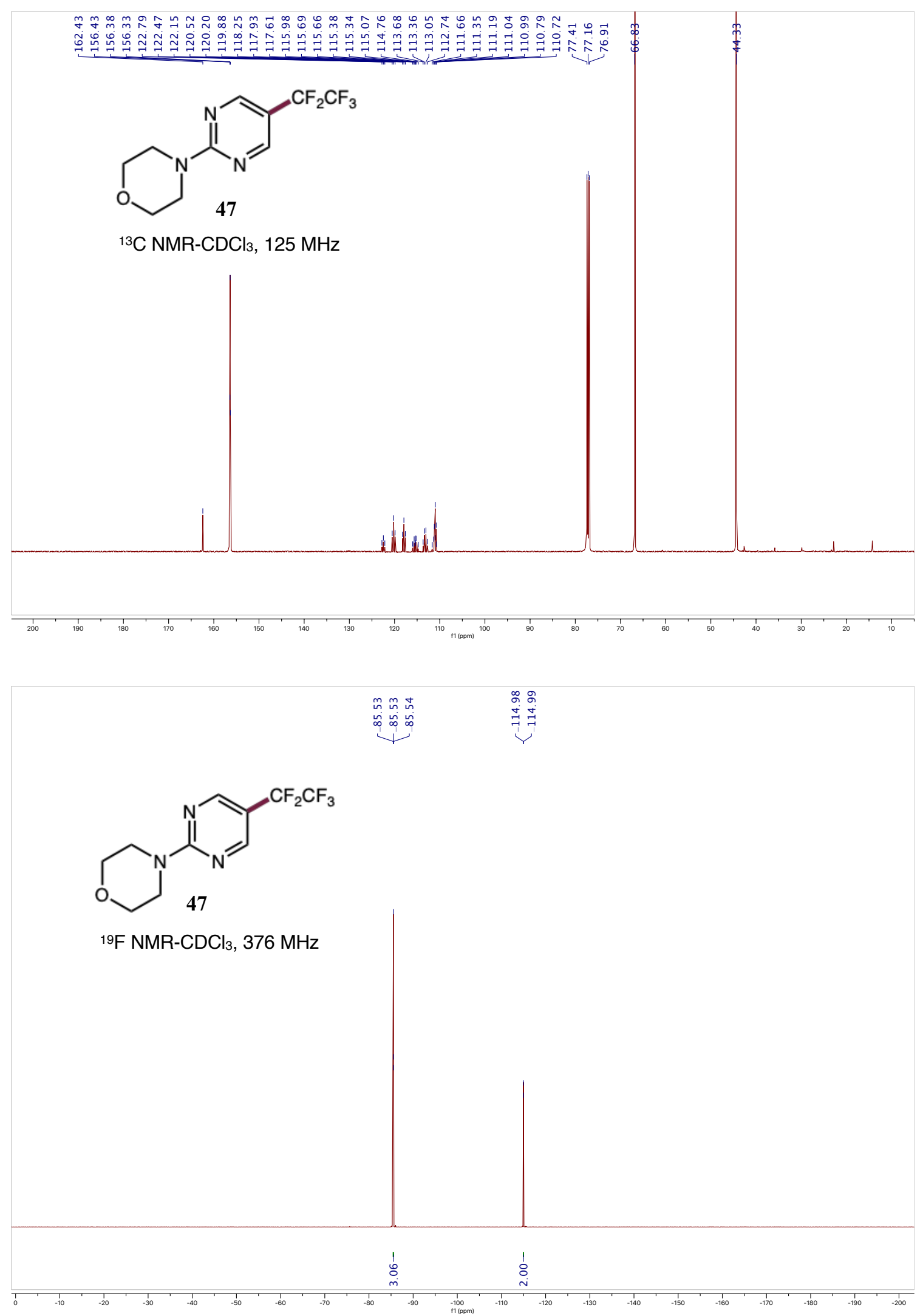

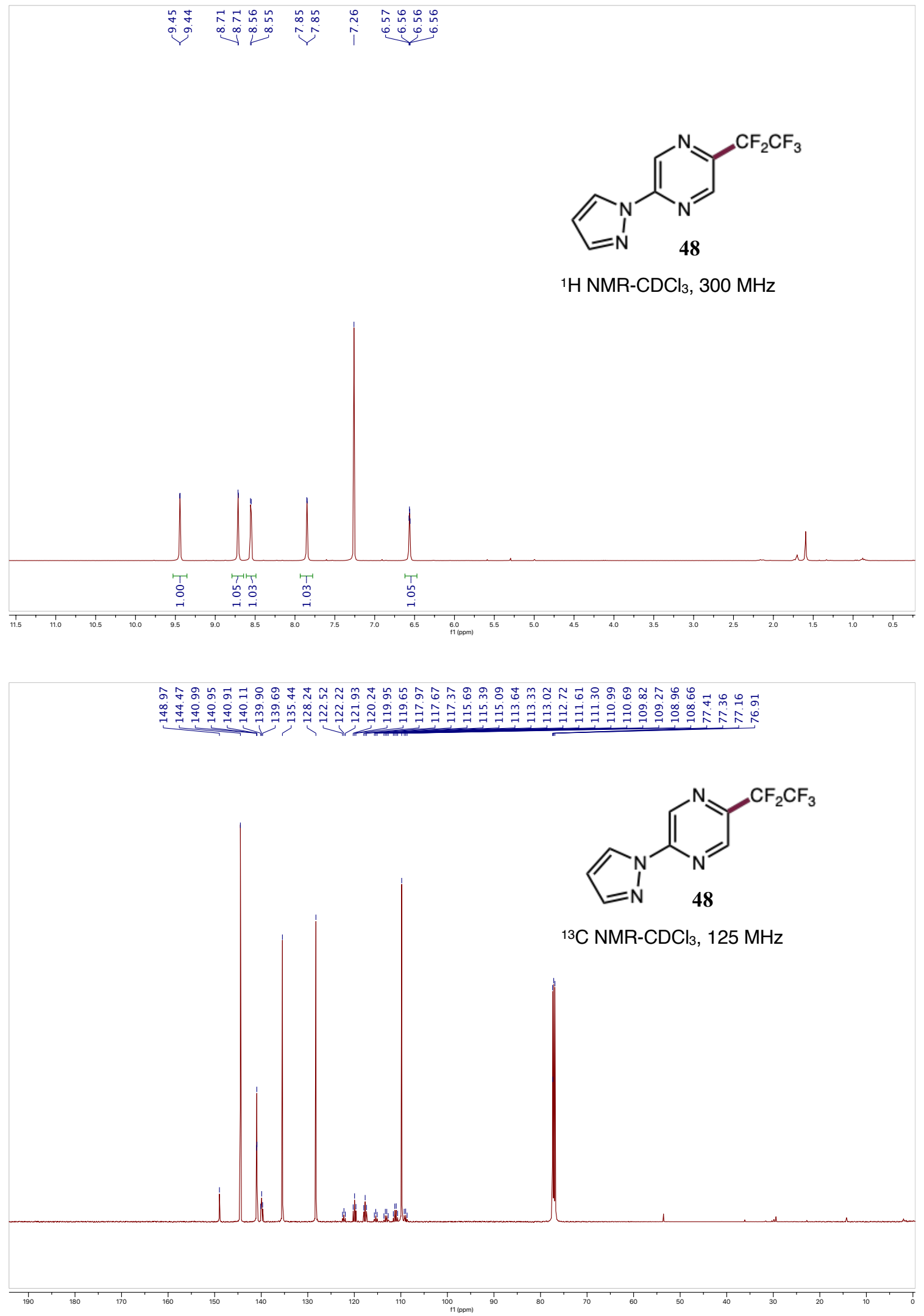


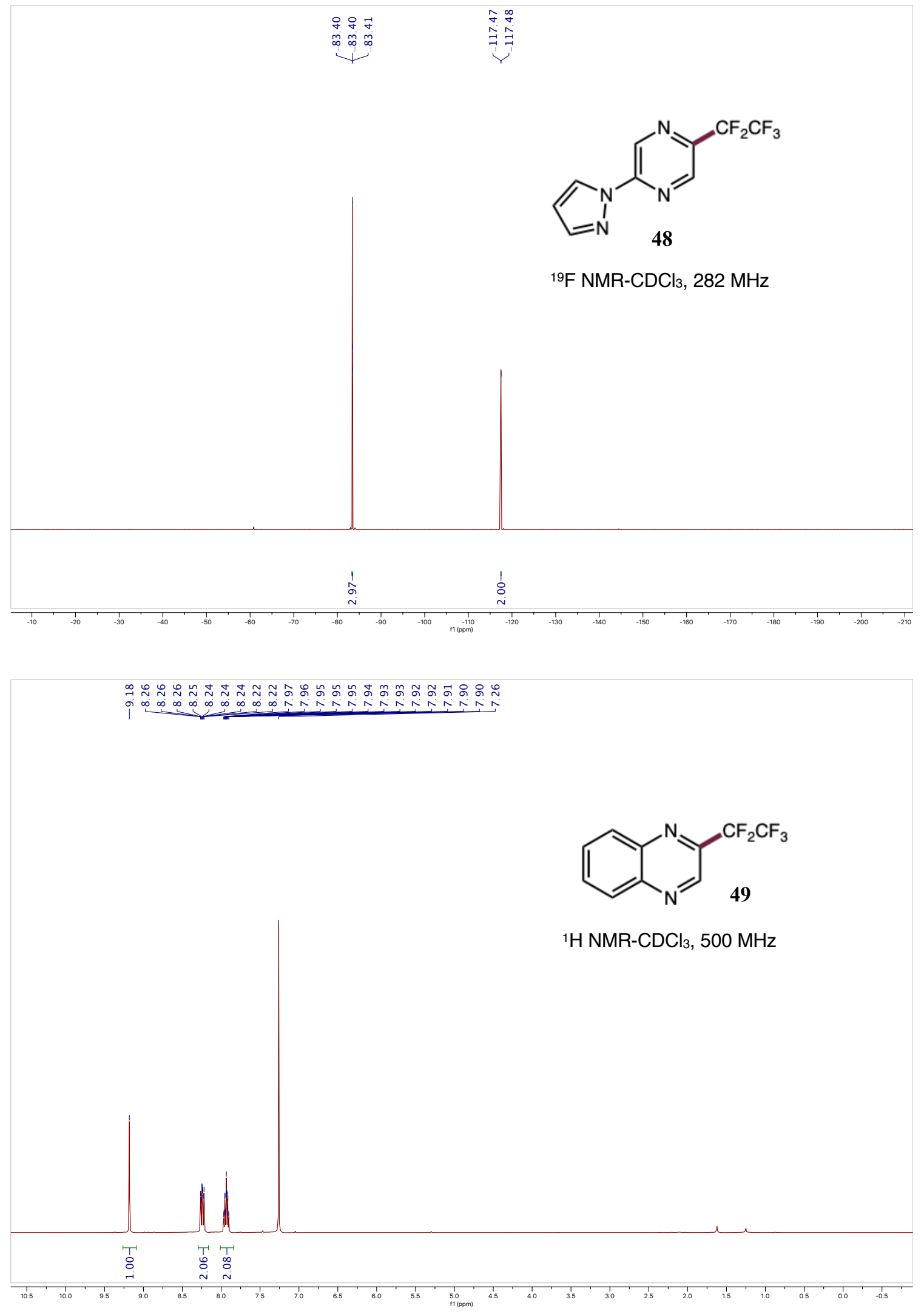



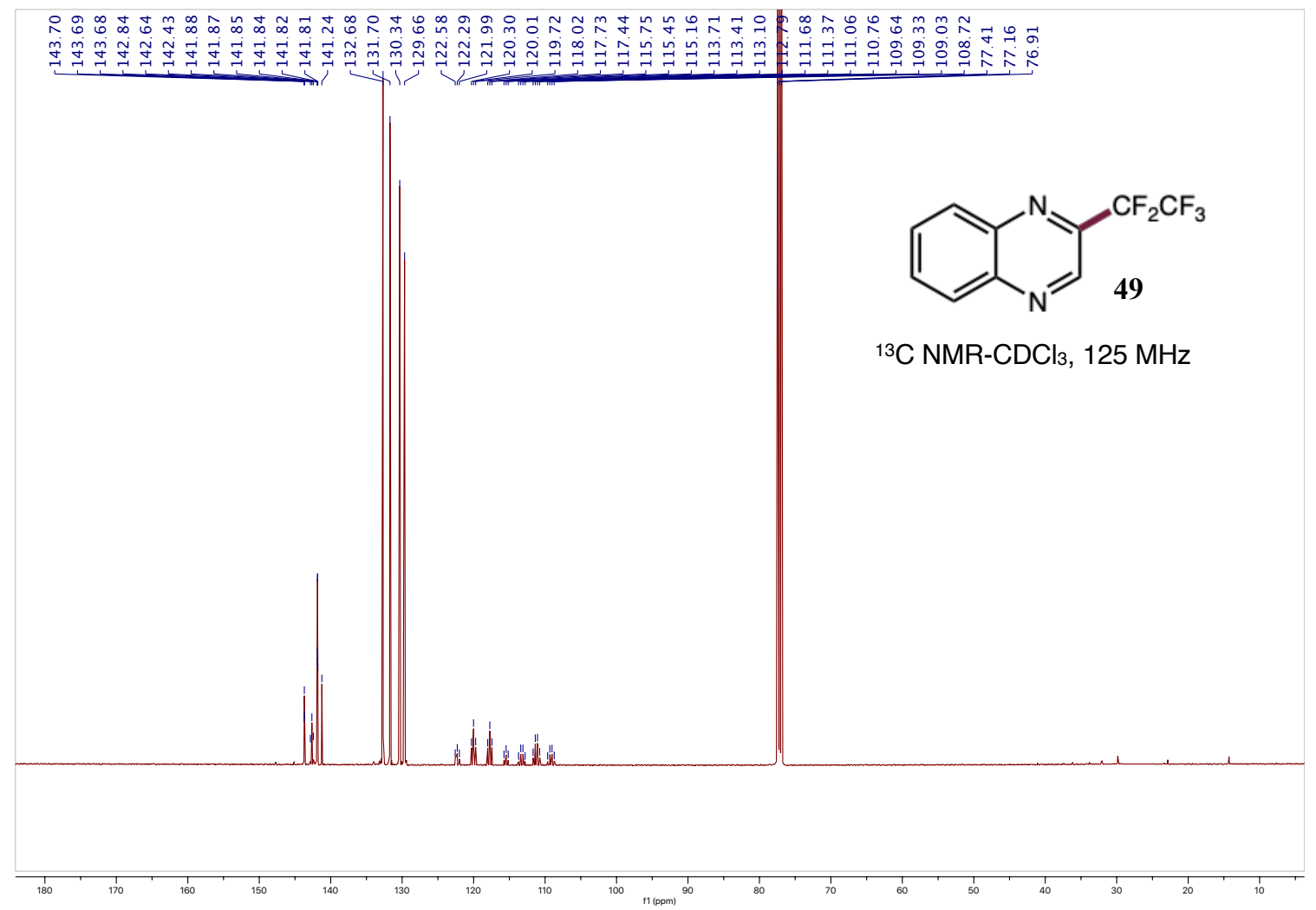

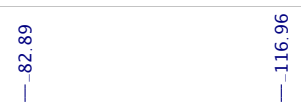

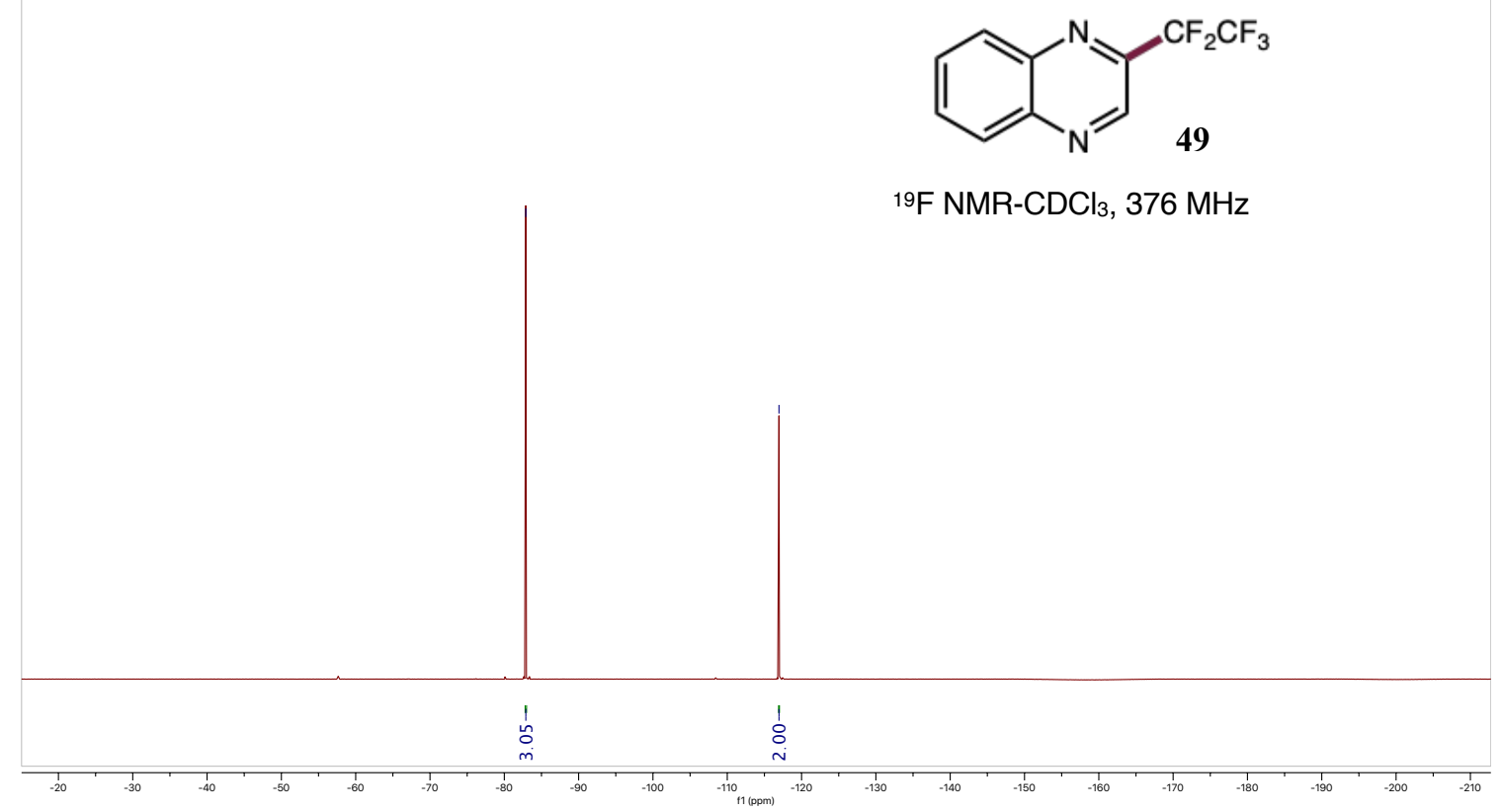




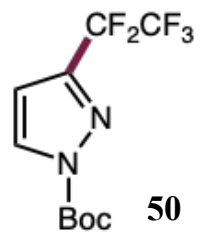

${ }^{1} \mathrm{H} \mathrm{NMR}-\mathrm{CDCl}_{3}, 500 \mathrm{MHz}$

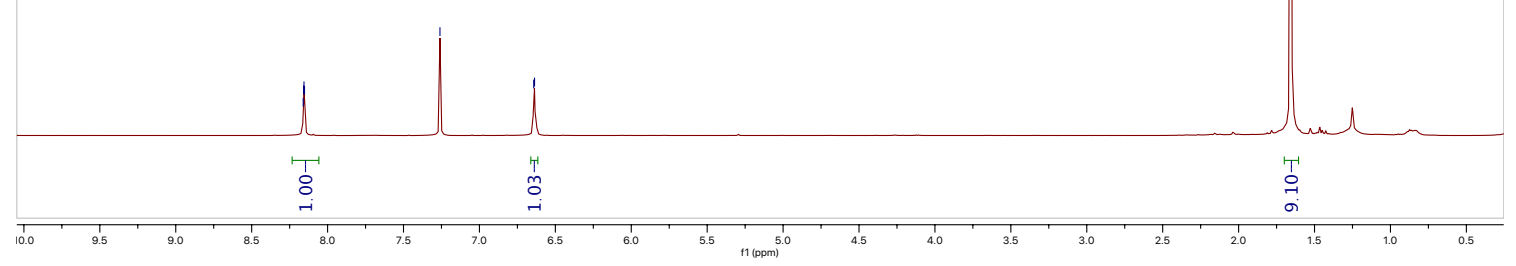

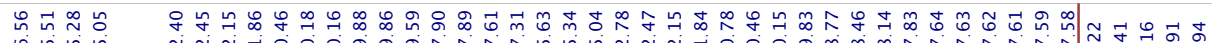

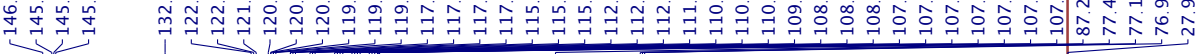

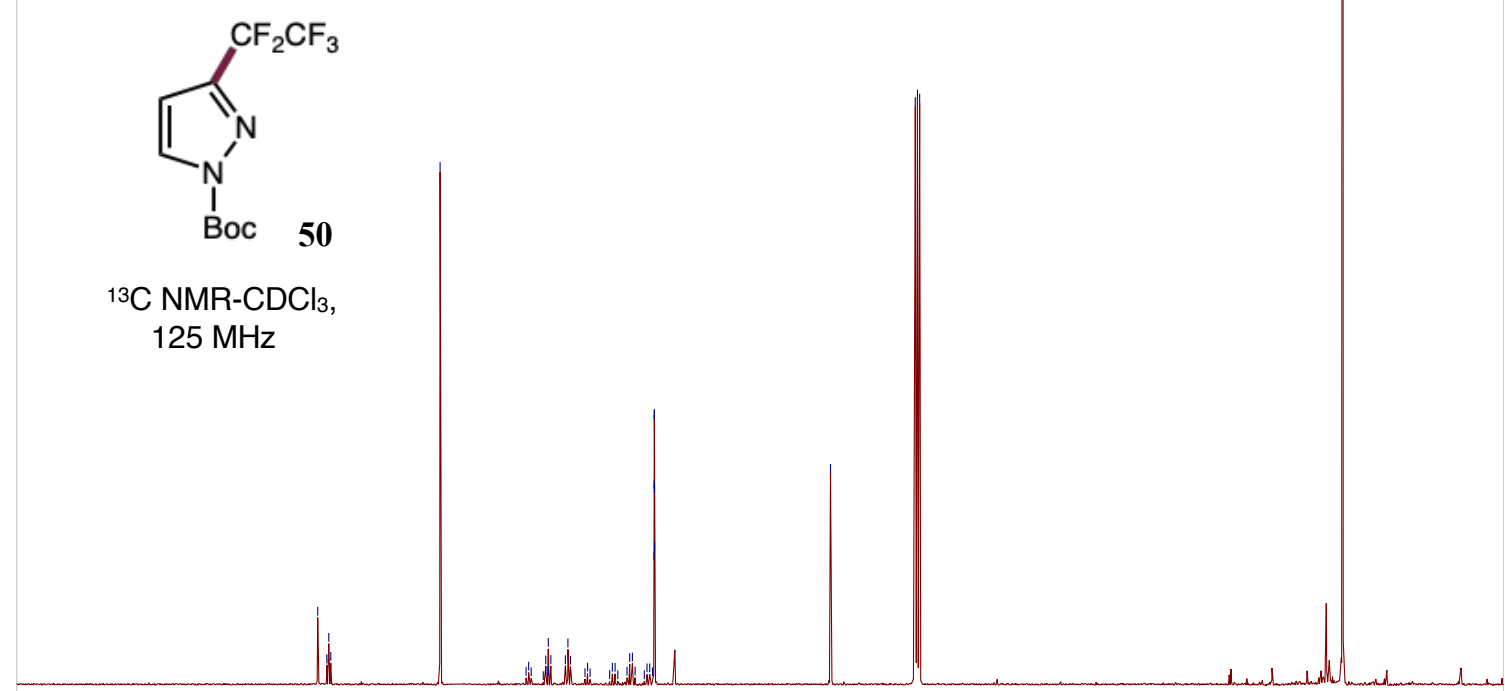




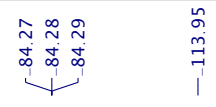

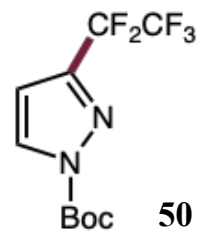

${ }^{19} \mathrm{~F} \mathrm{NMR-CDCl} 3,282 \mathrm{MHz}$

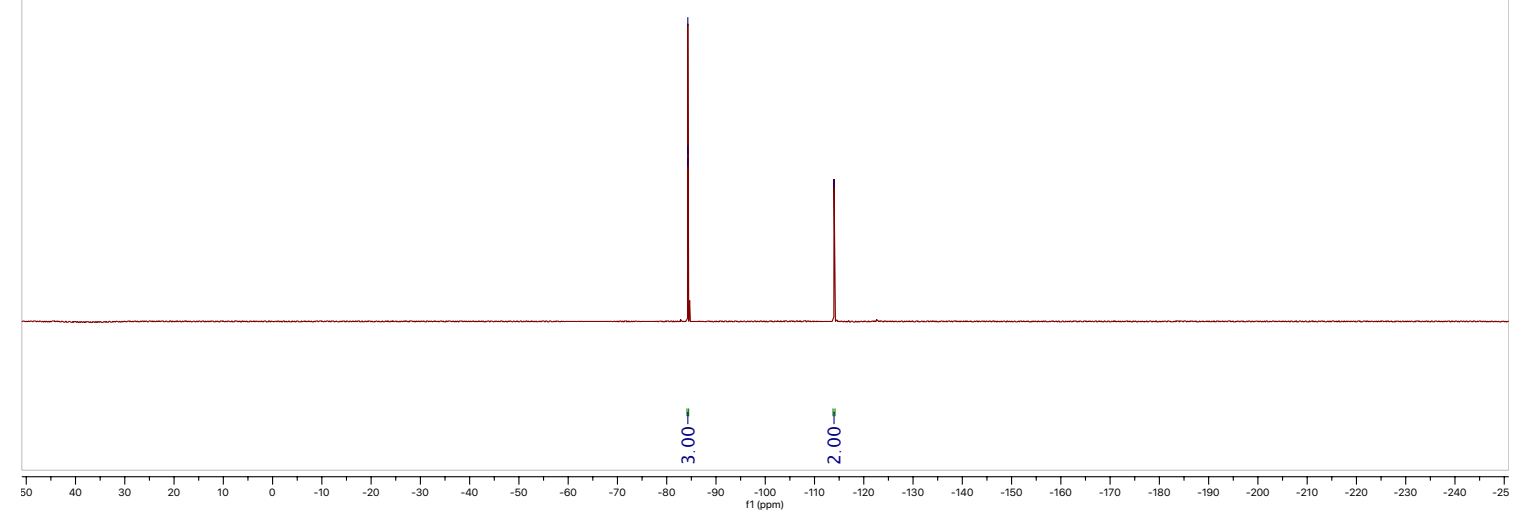

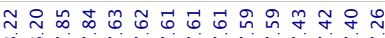

Niñ

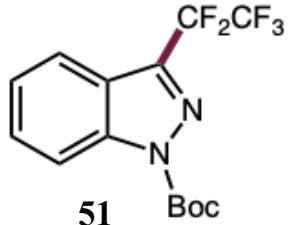

${ }^{1} \mathrm{H} \mathrm{NMR}-\mathrm{CDCl}_{3}, 500 \mathrm{MHz}$

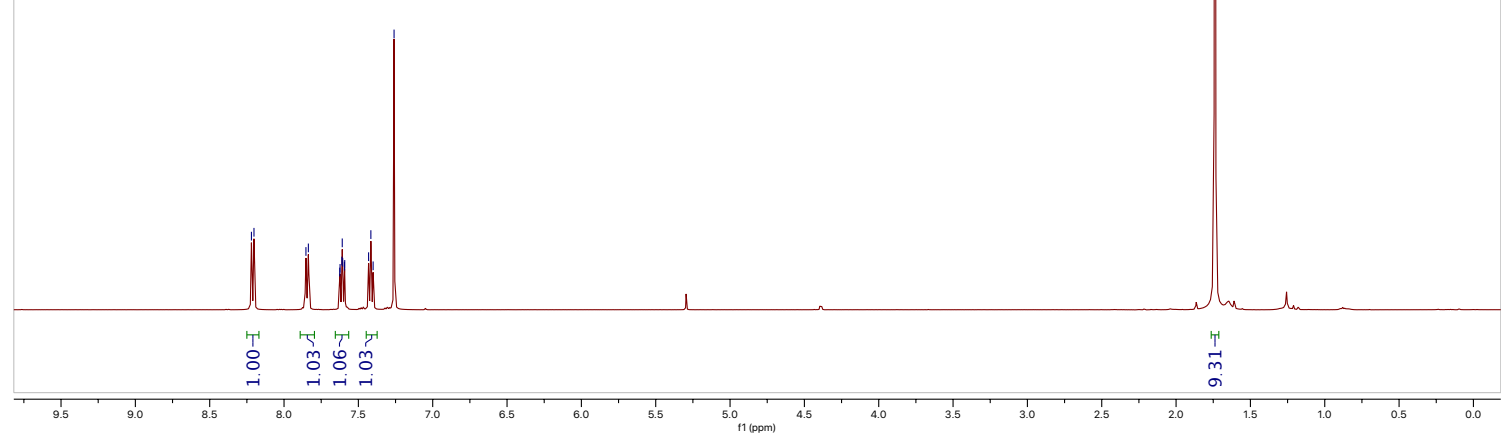



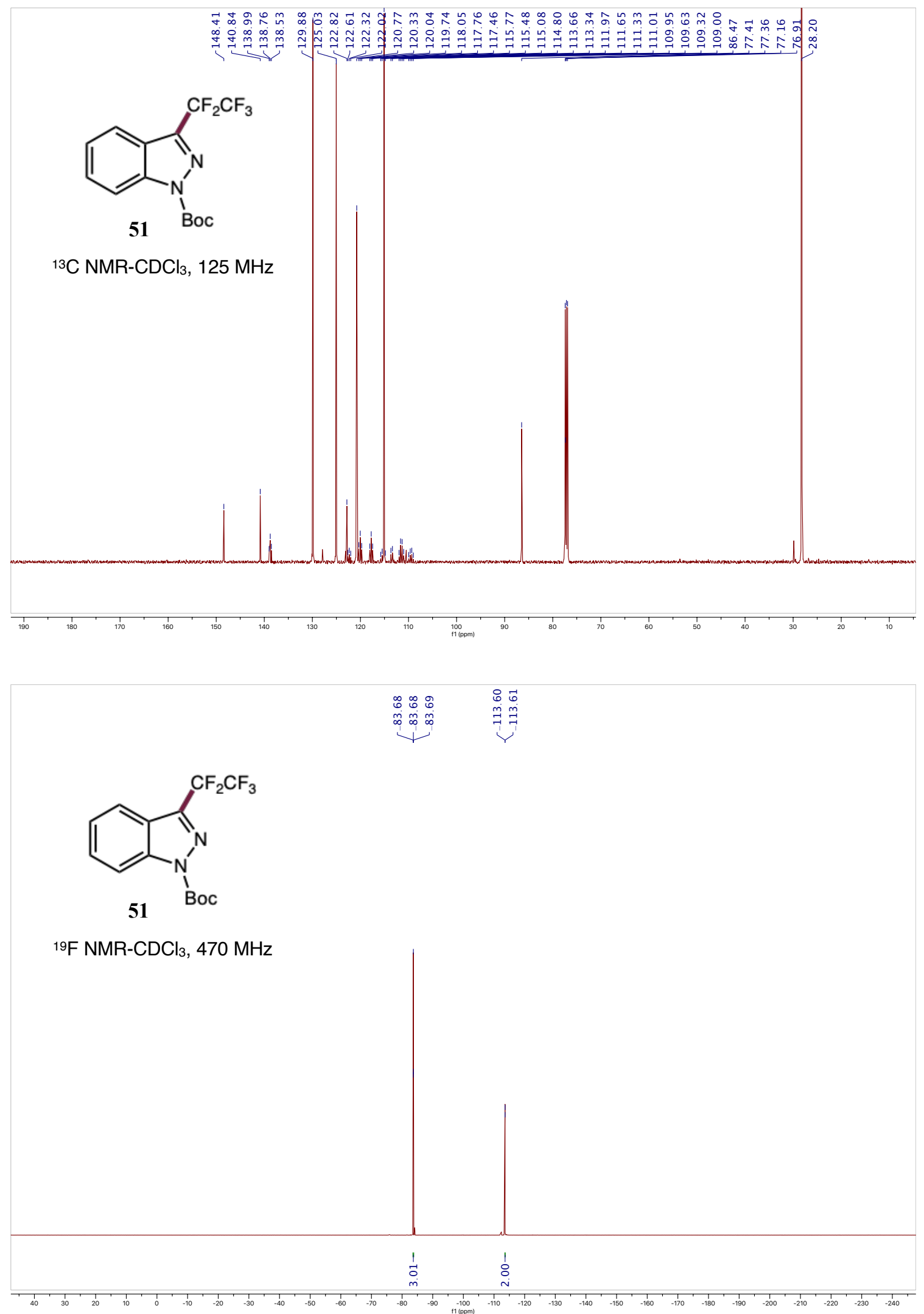

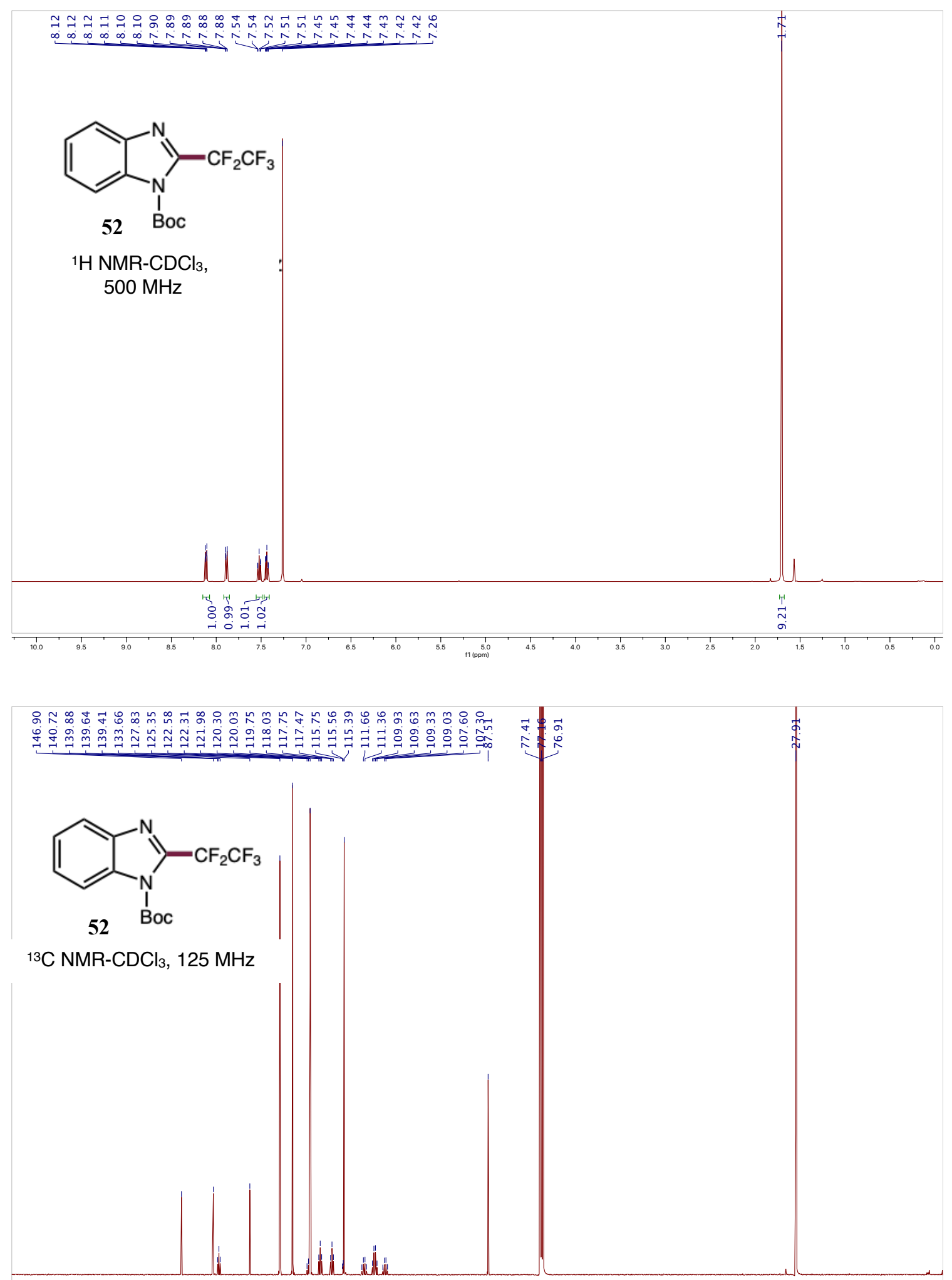


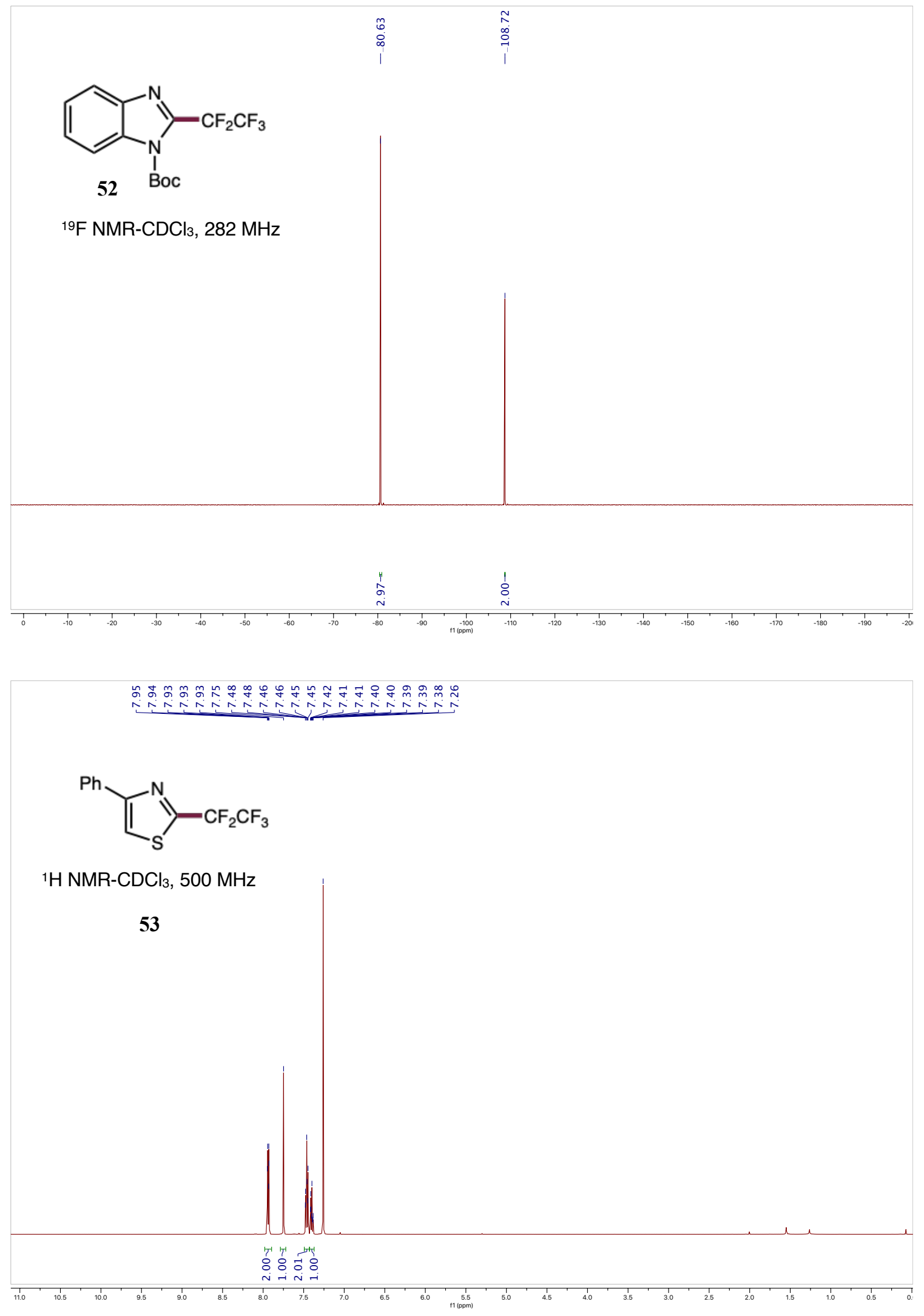



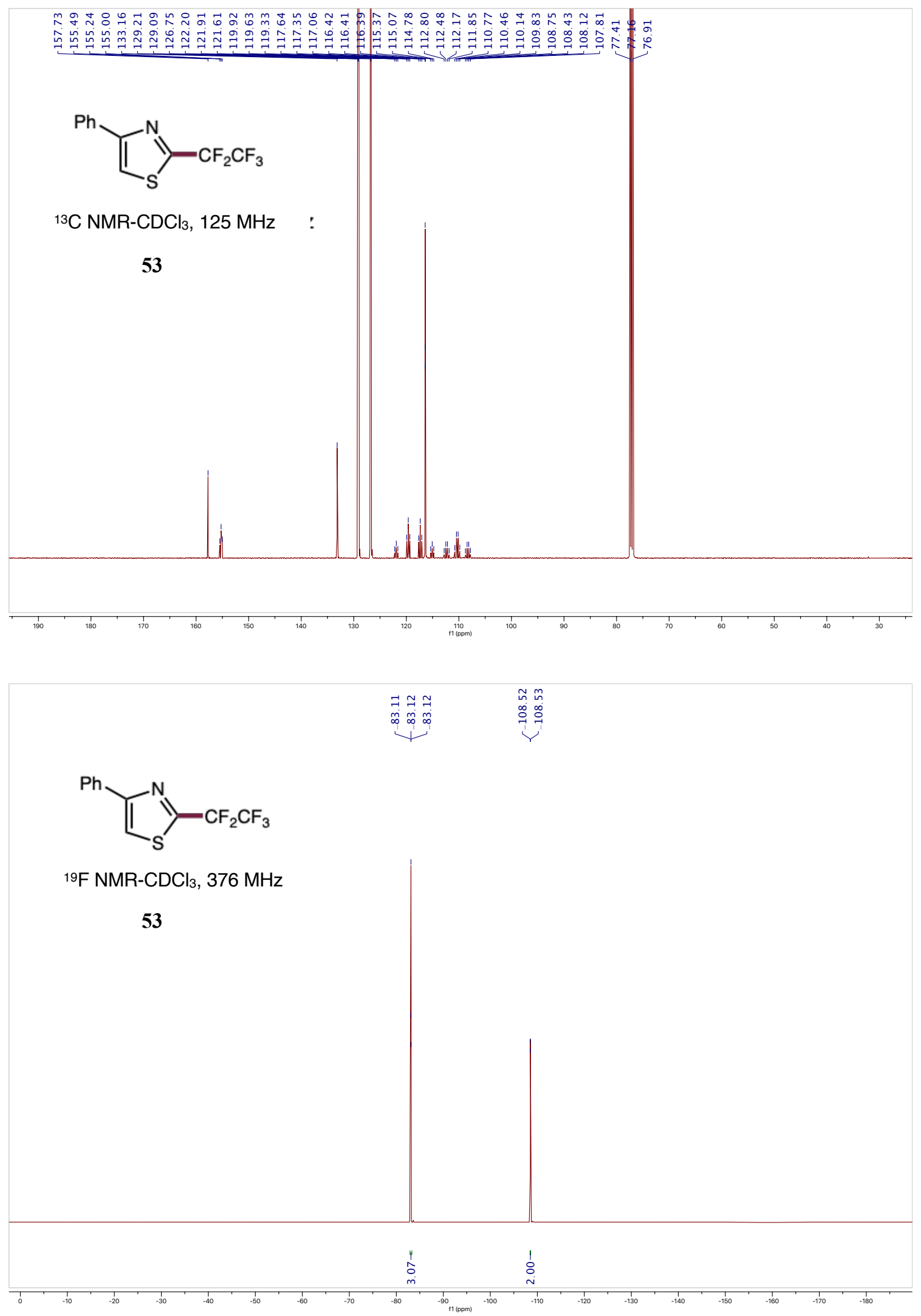


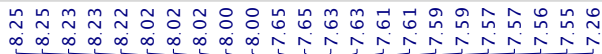

$\left(-\mathrm{CF}_{2} \mathrm{CF}_{3}\right.$

${ }^{1} \mathrm{H} \mathrm{NMR}-\mathrm{CDCl}_{3}, 400 \mathrm{MHz}$

54

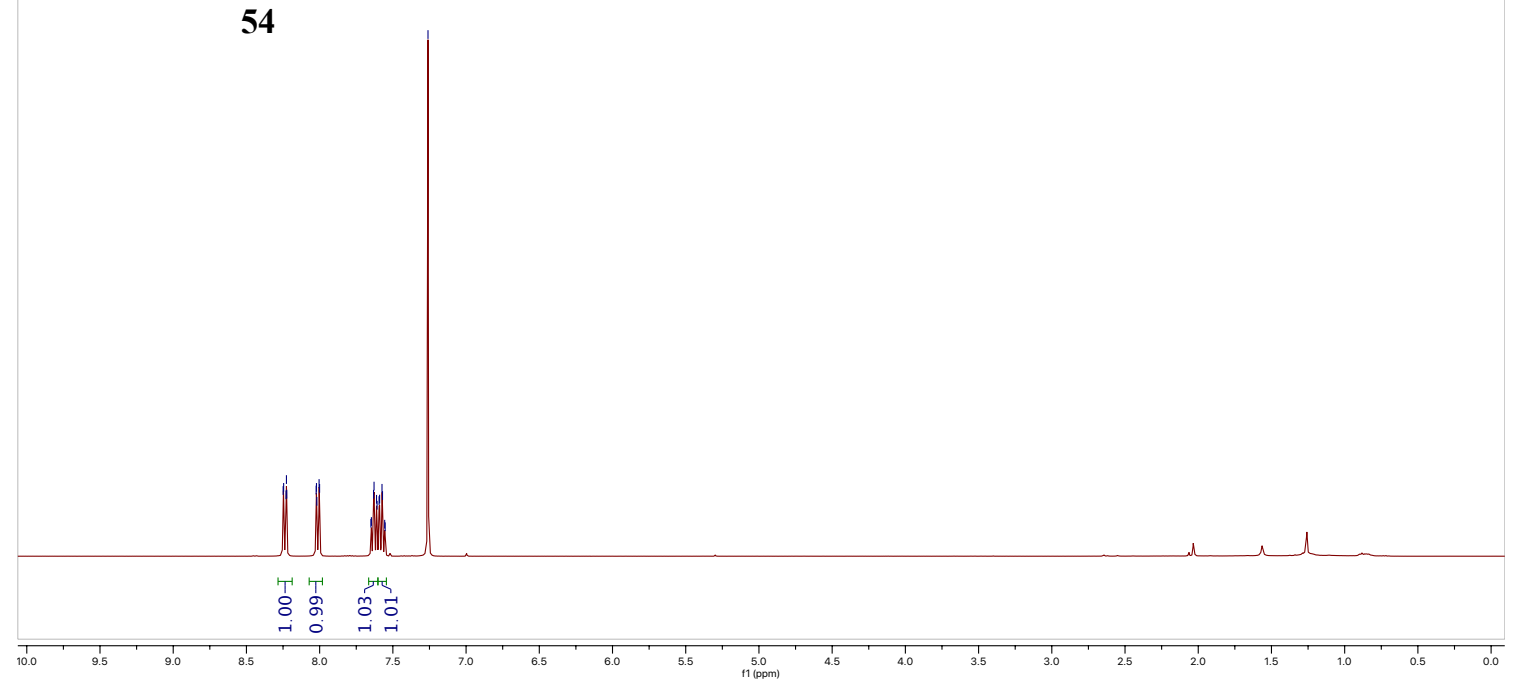

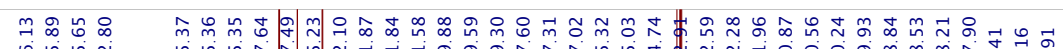

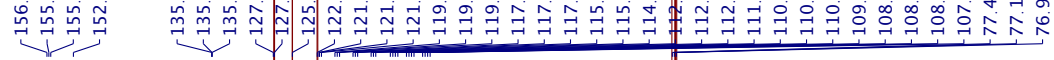<smiles>FC(F)(F)C(F)(F)c1nc2ccccc2s1</smiles>

${ }^{13} \mathrm{C} \mathrm{NMR-CDCl} 3,125 \mathrm{MHz}$ 54
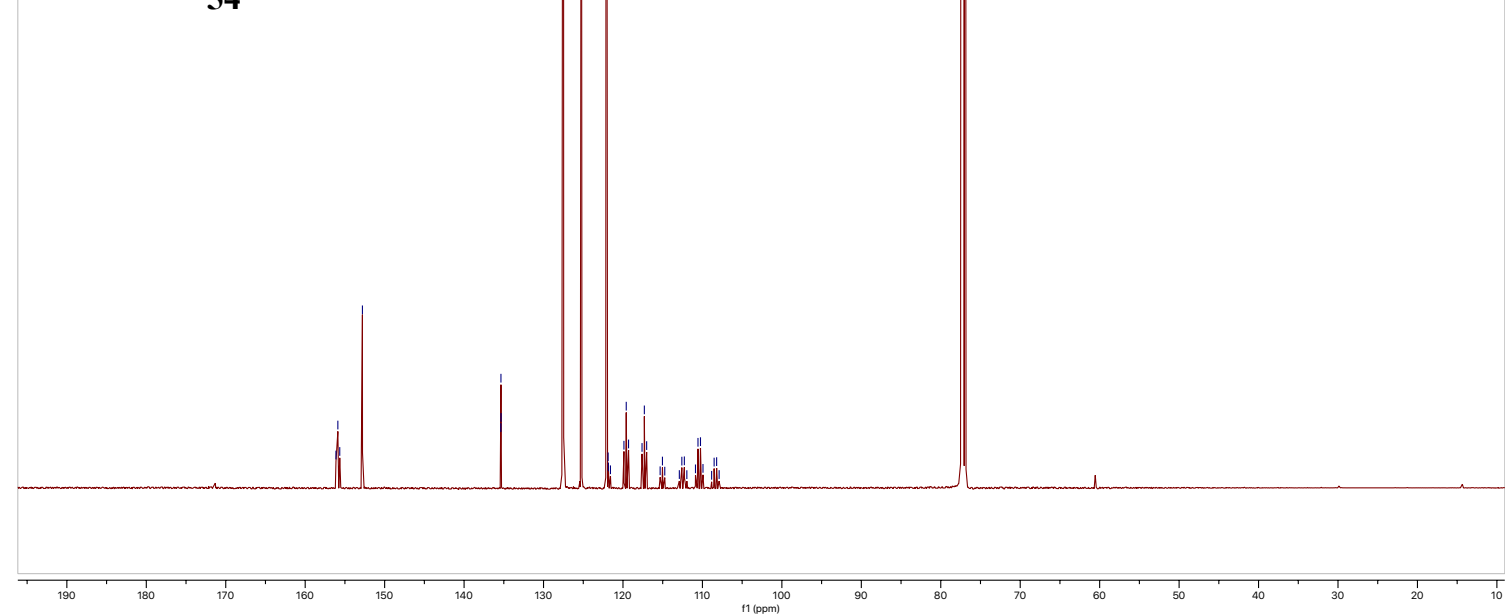


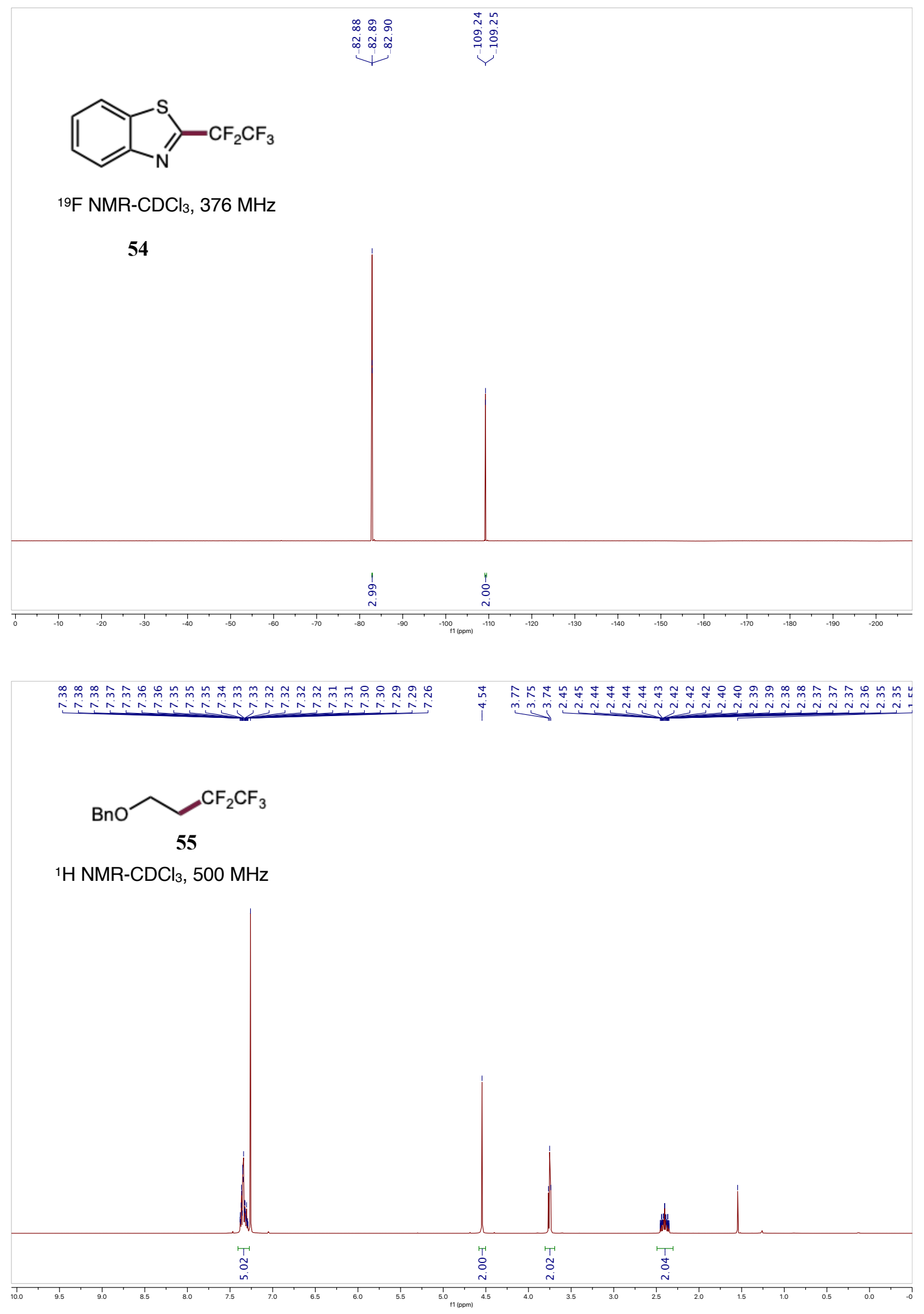



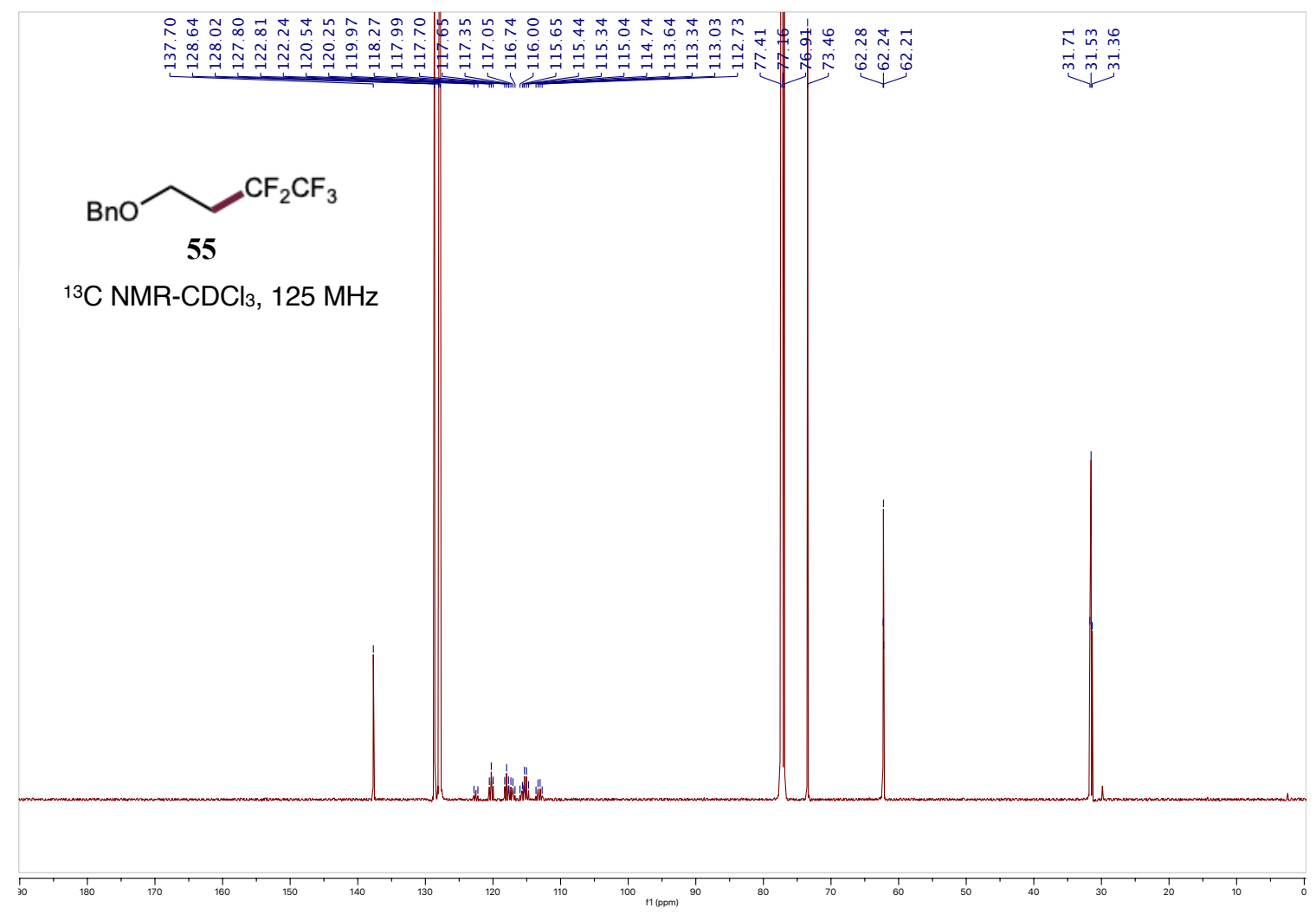

1
$\infty$
$\infty$
$\infty$
$\infty$

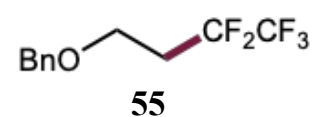

${ }^{19} \mathrm{~F}$ NMR-CDCl $3,470 \mathrm{MHz}$

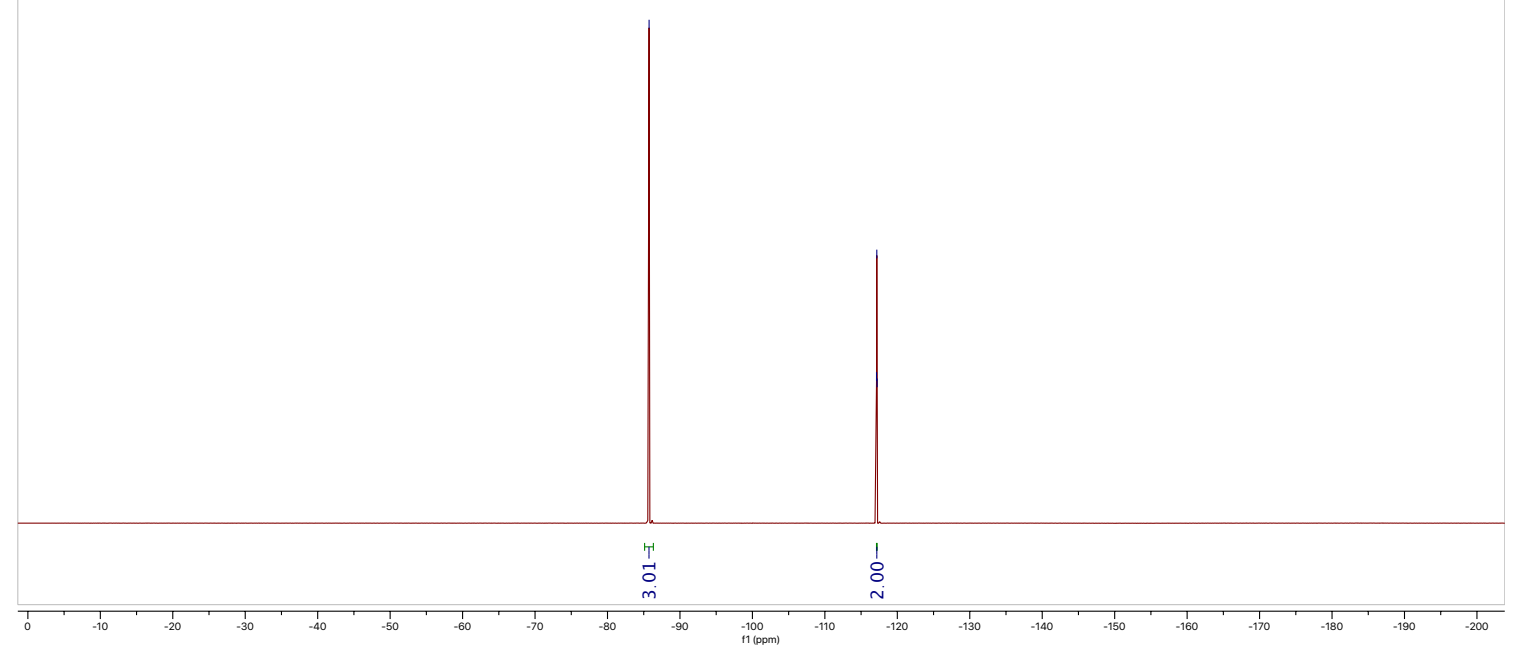




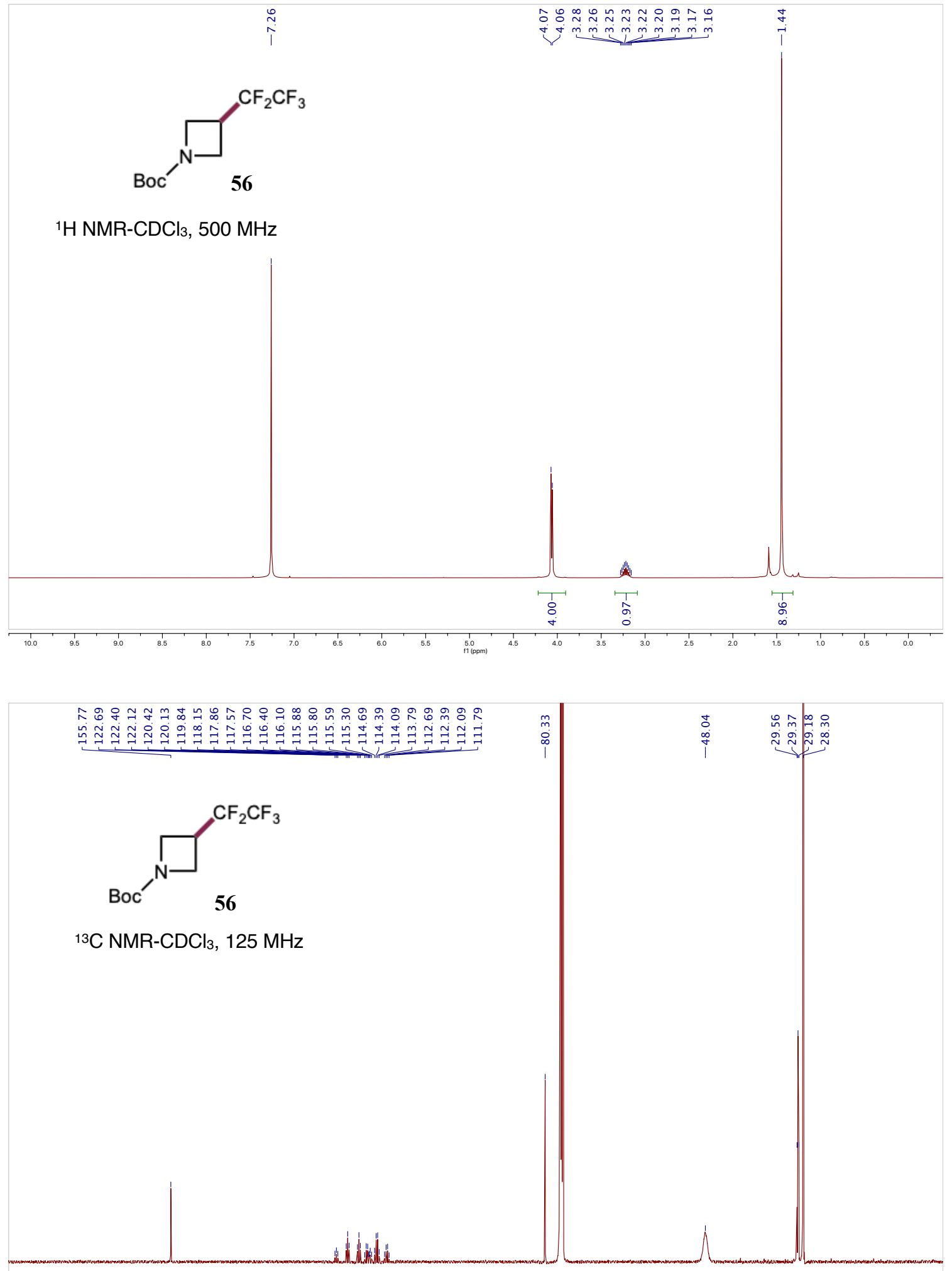



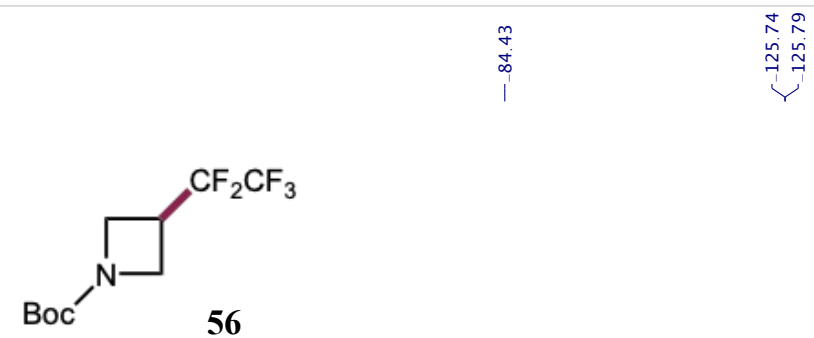

19F NMR-CDCl $3,282 \mathrm{MHz}$

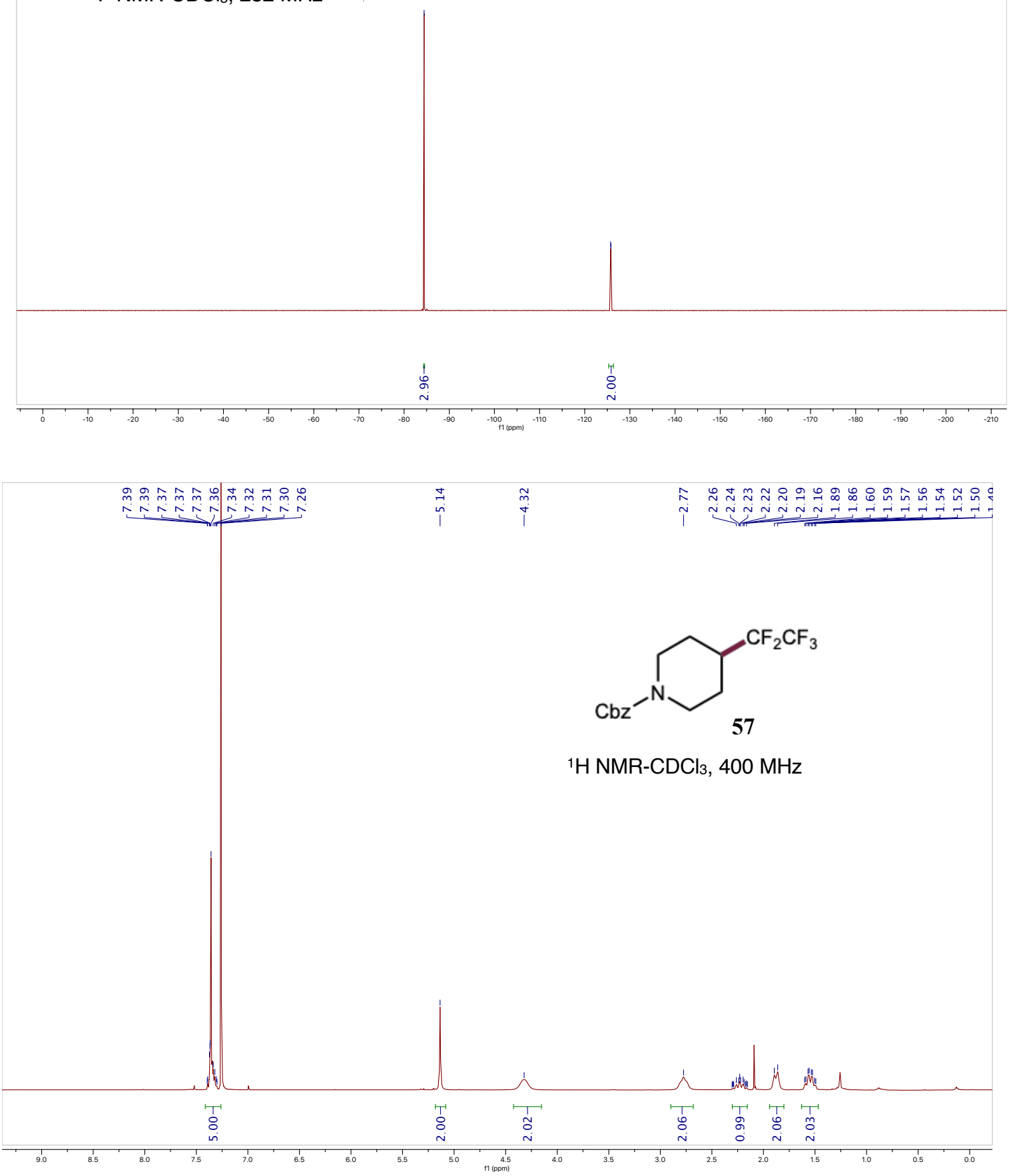



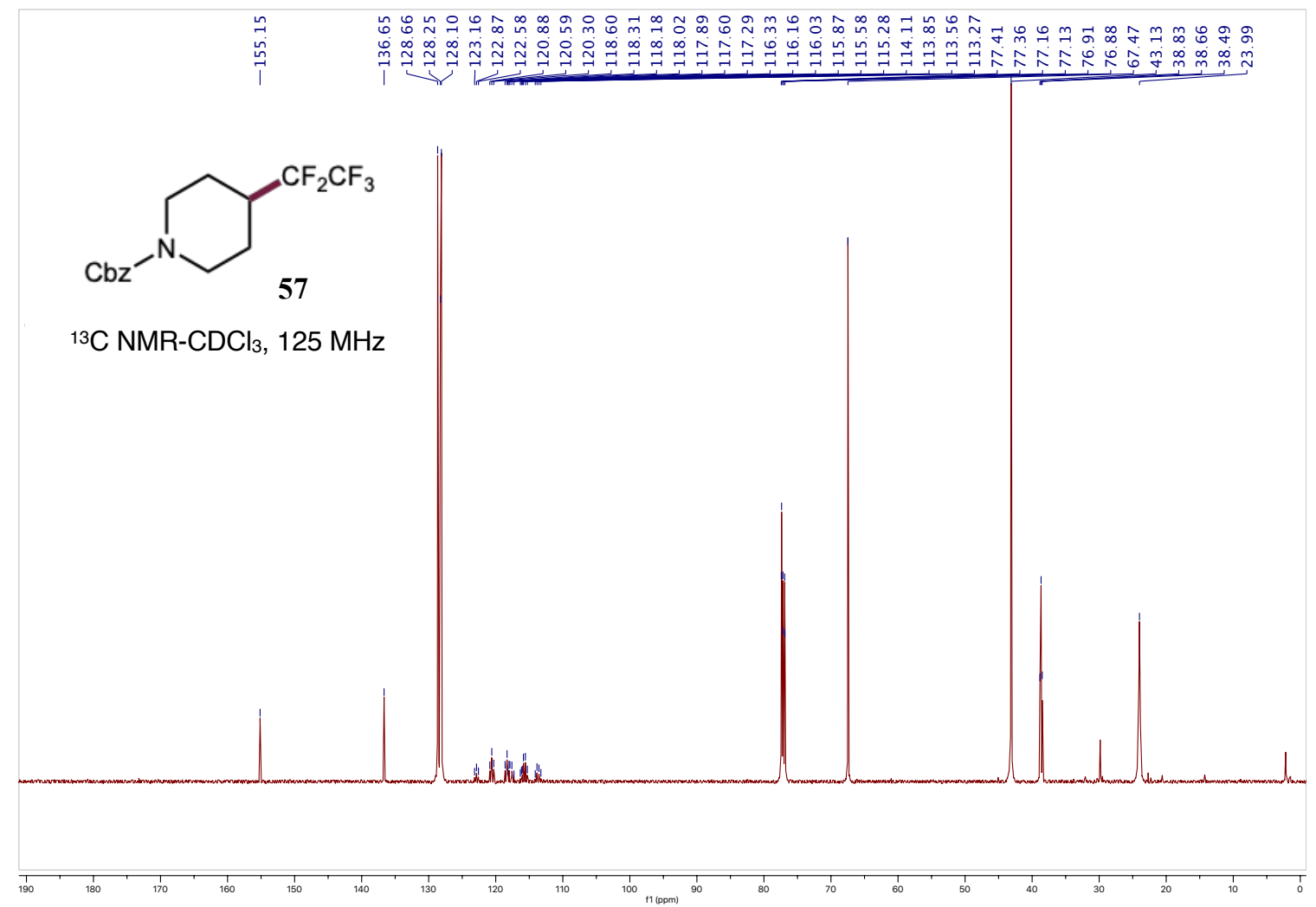

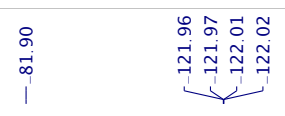

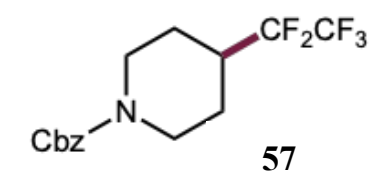

19F NMR-CDCl $3,282 \mathrm{MHz}$

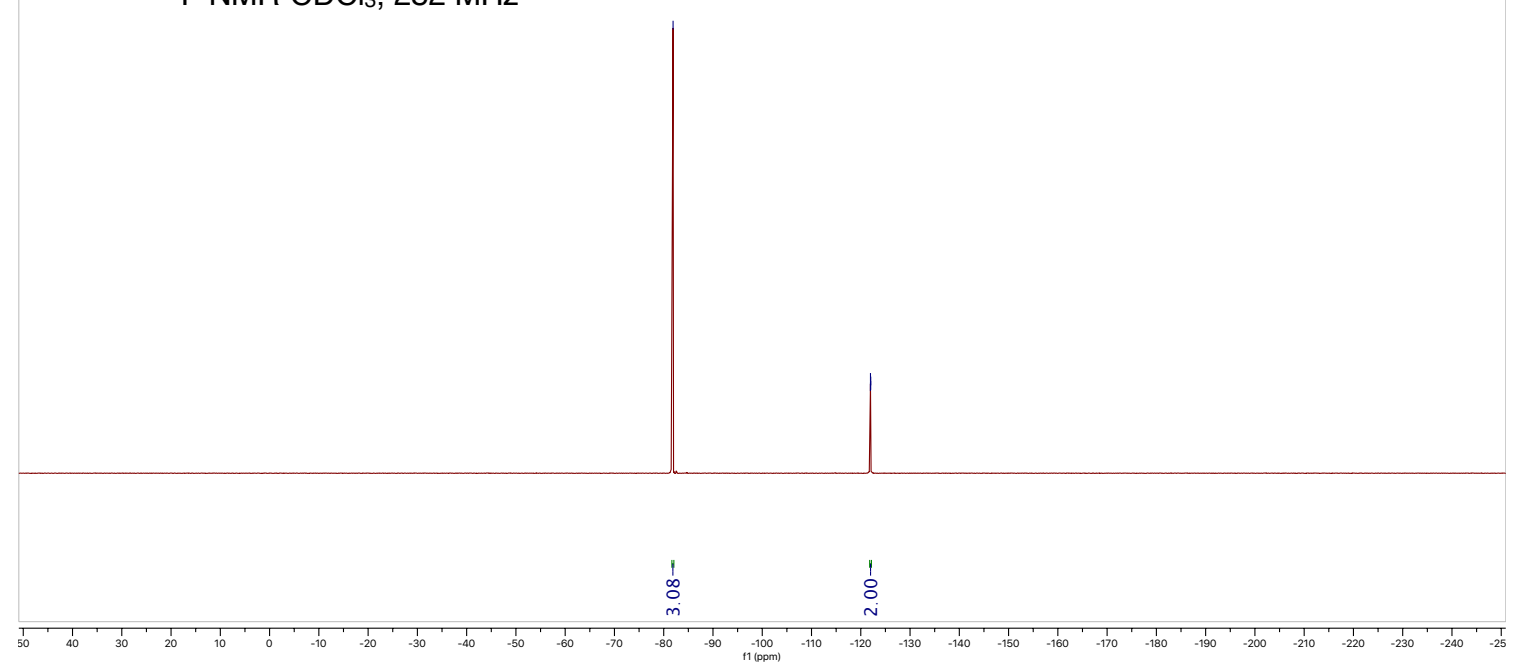




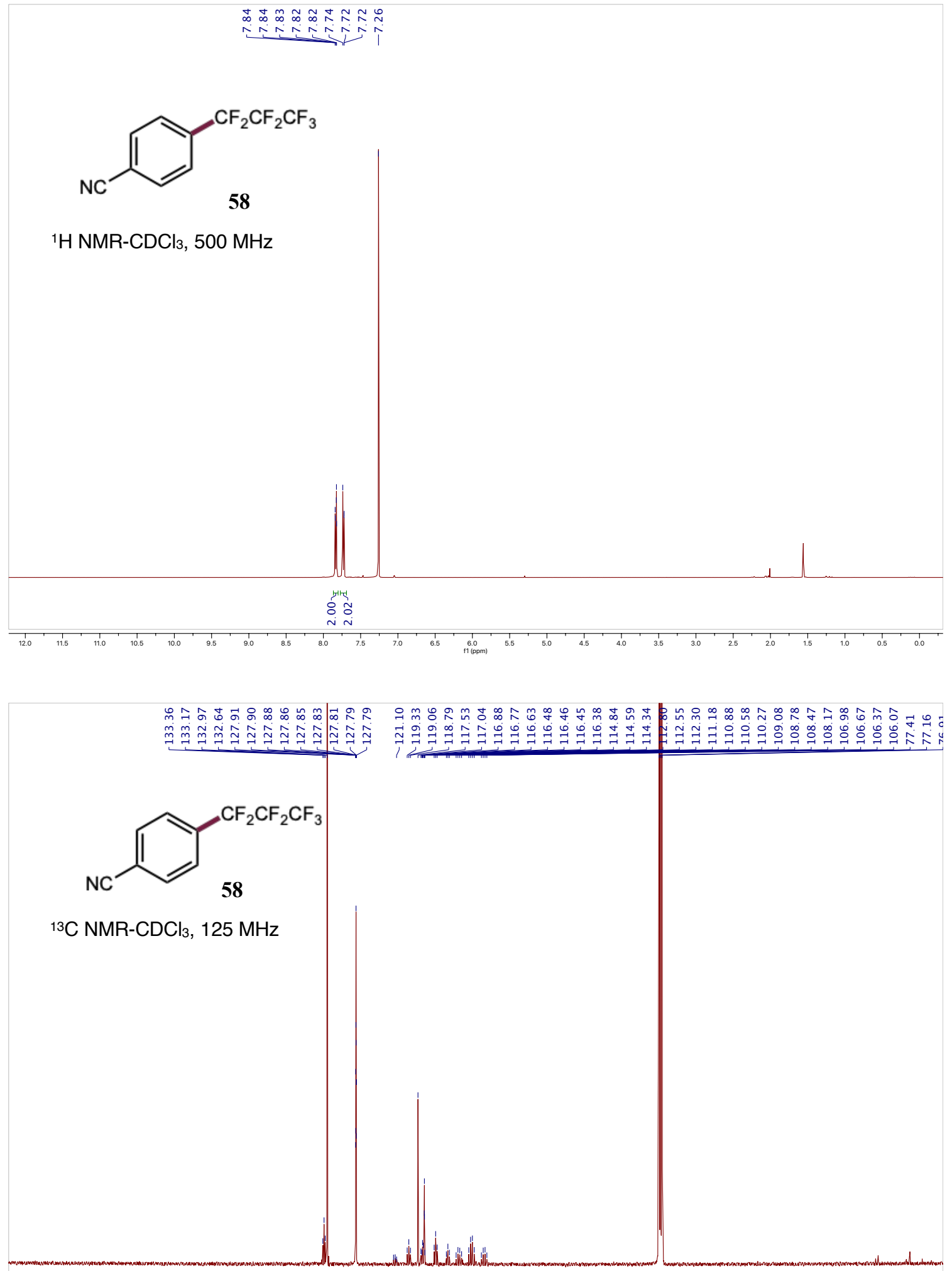




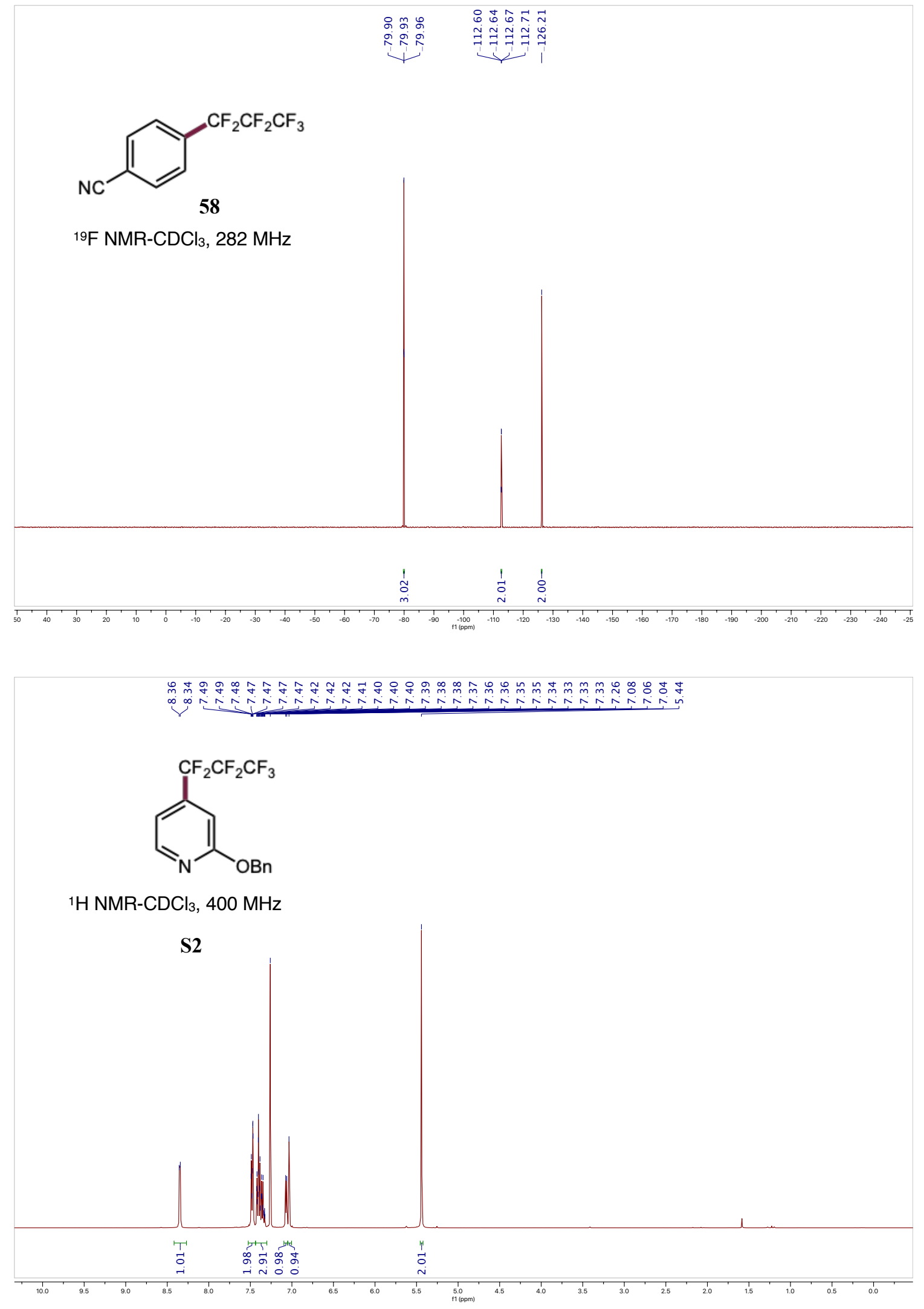



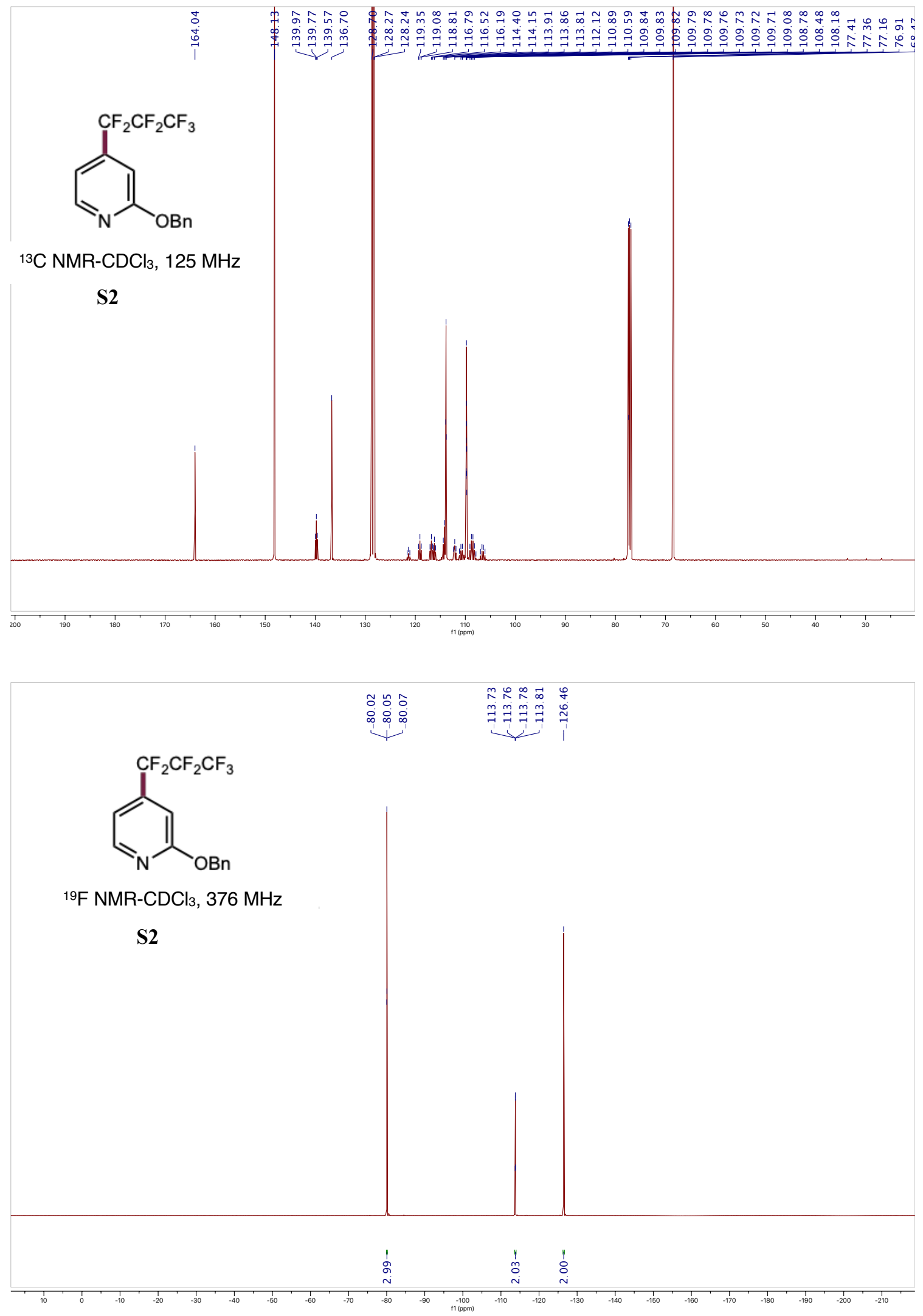

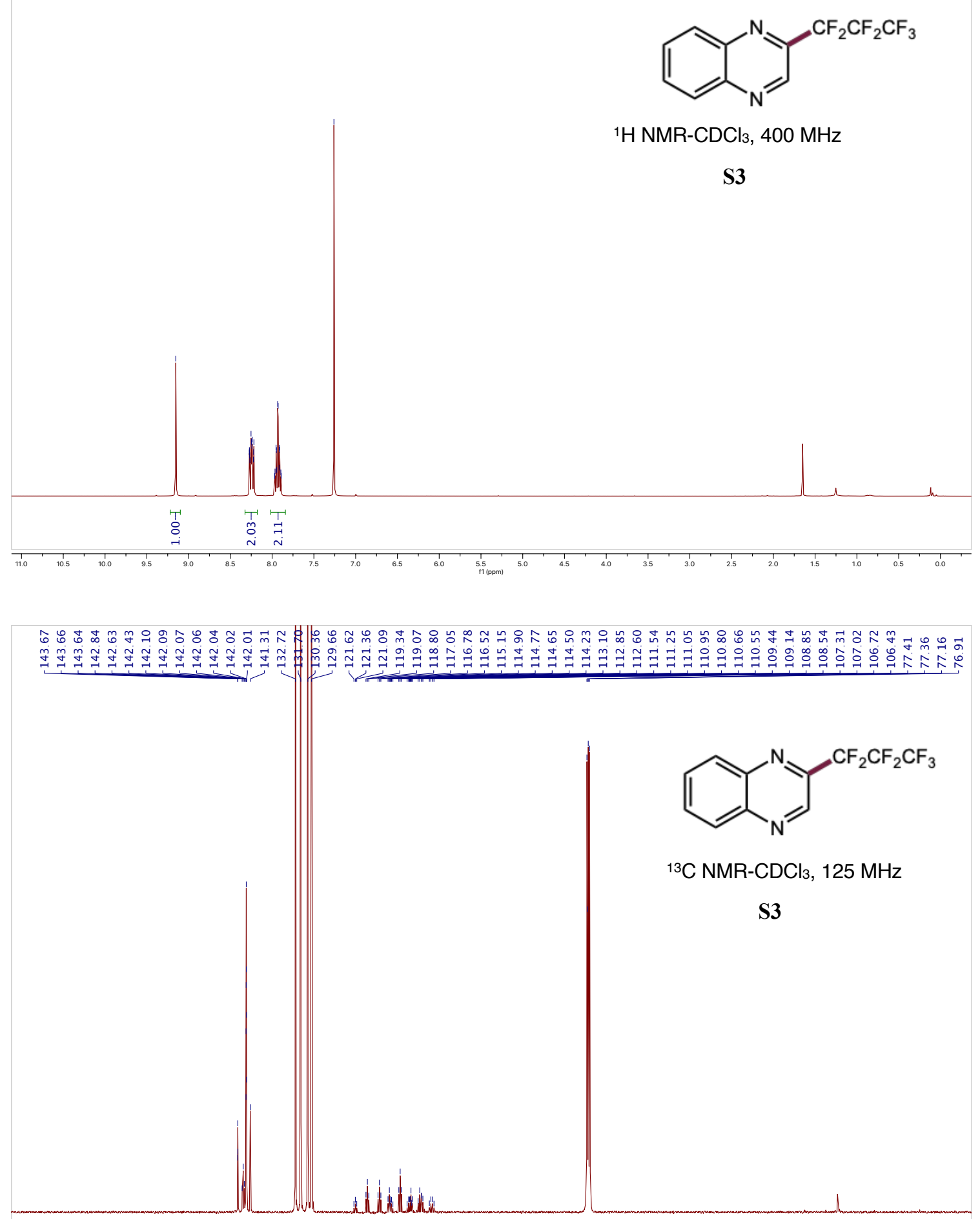


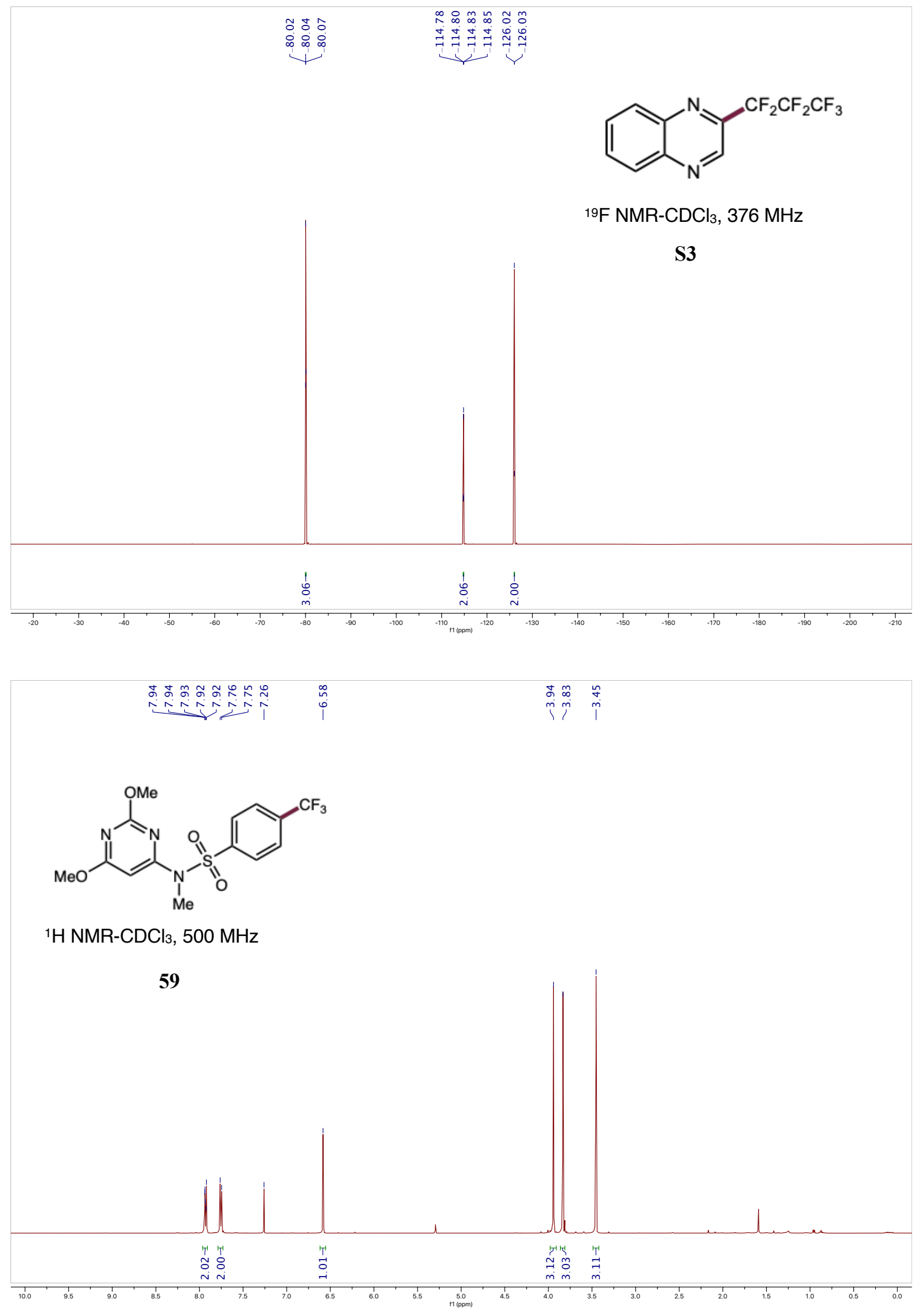



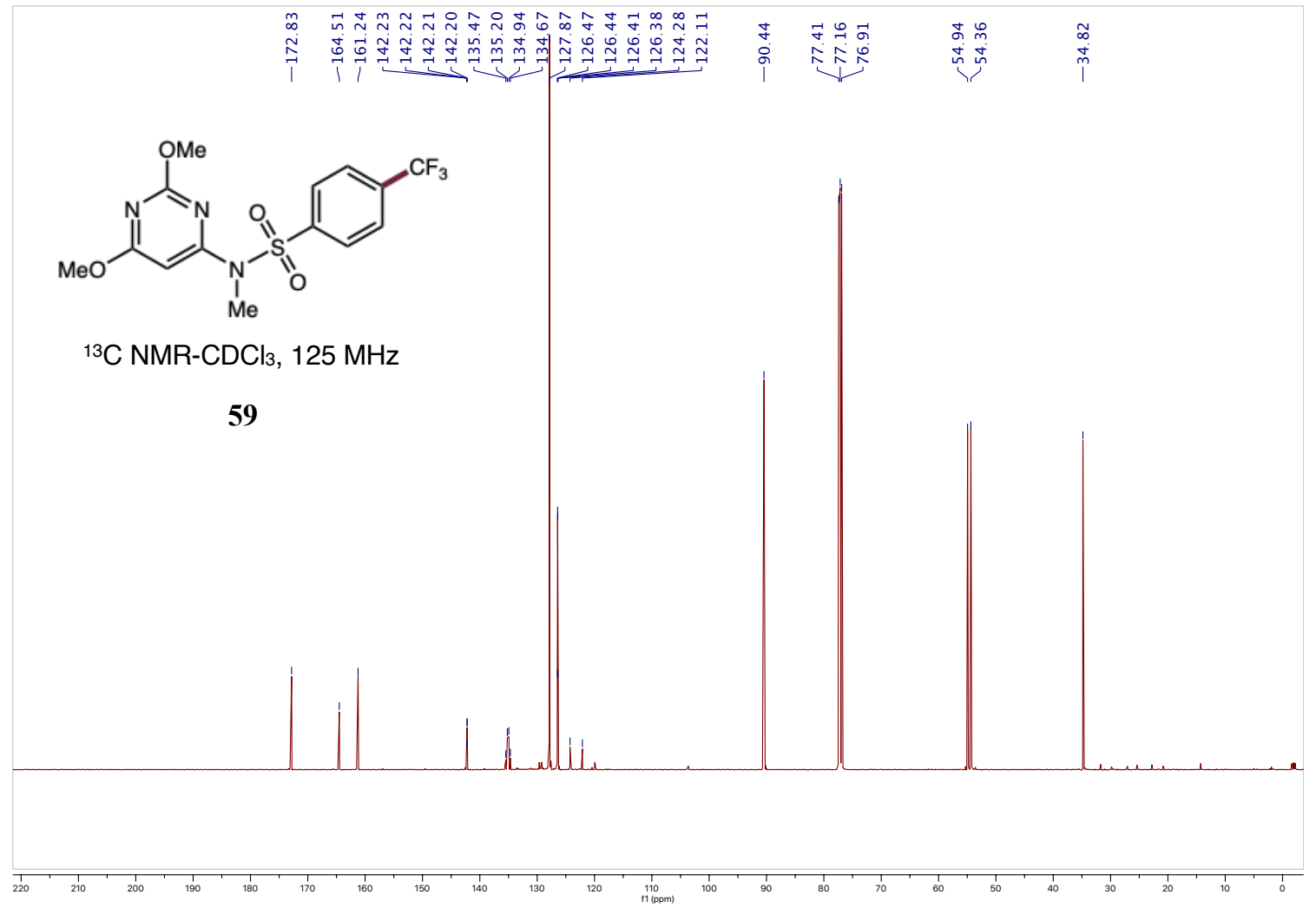

$\stackrel{+}{\sim}$
$n$
0
1

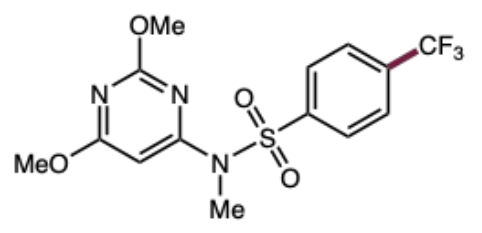

${ }^{19} \mathrm{~F} \mathrm{NMR-CDCl} 3,282 \mathrm{MHz}$

59 

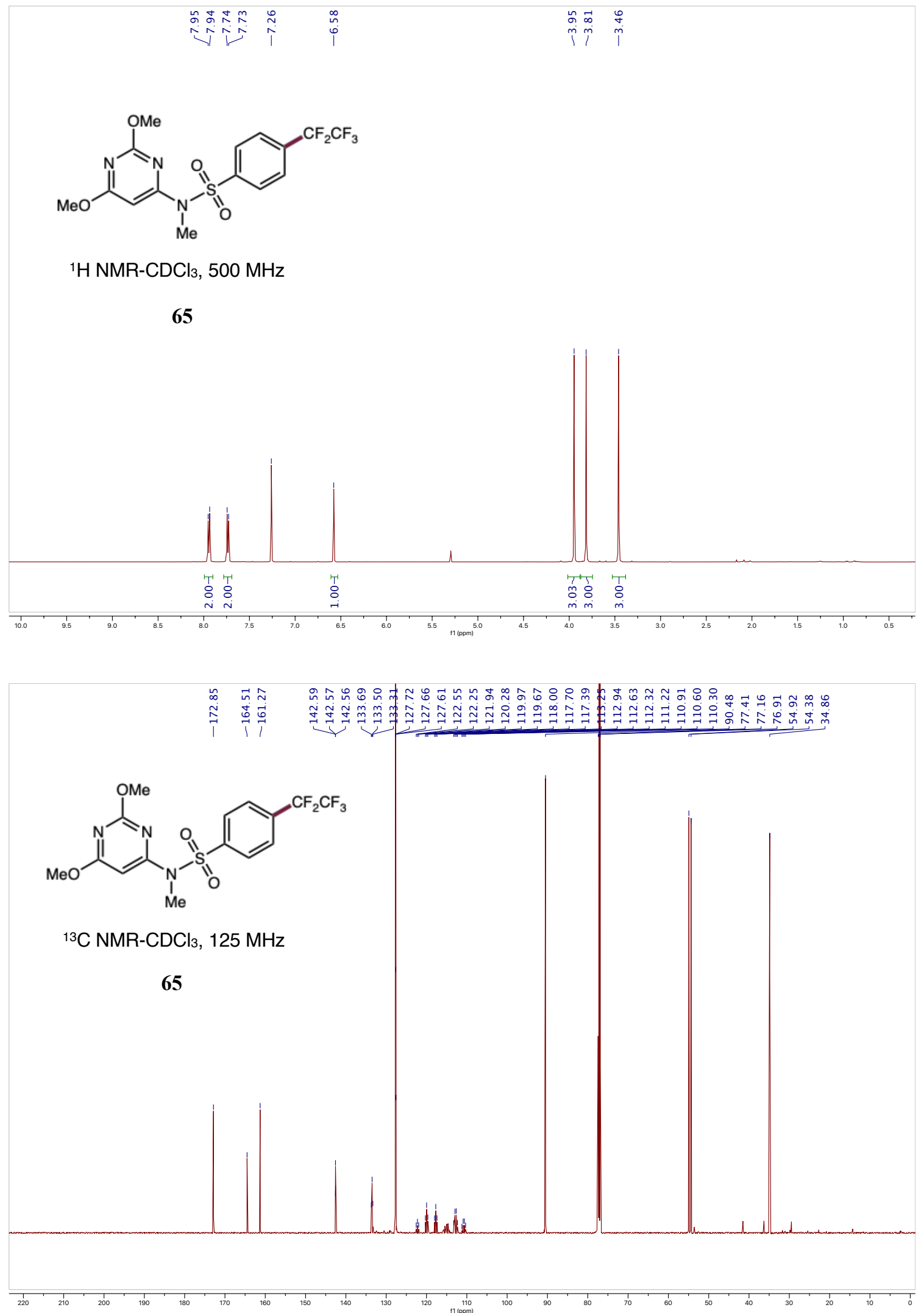


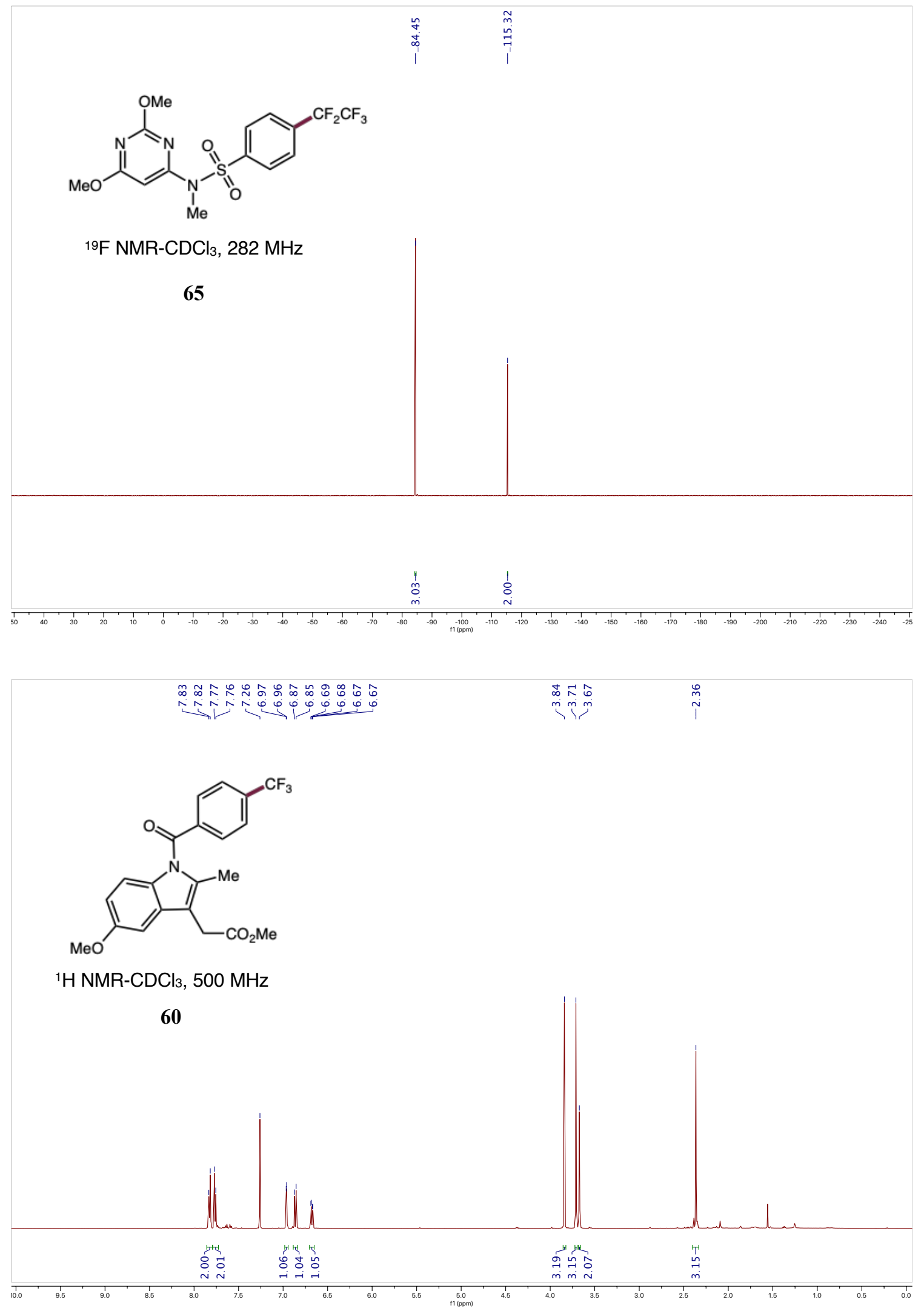



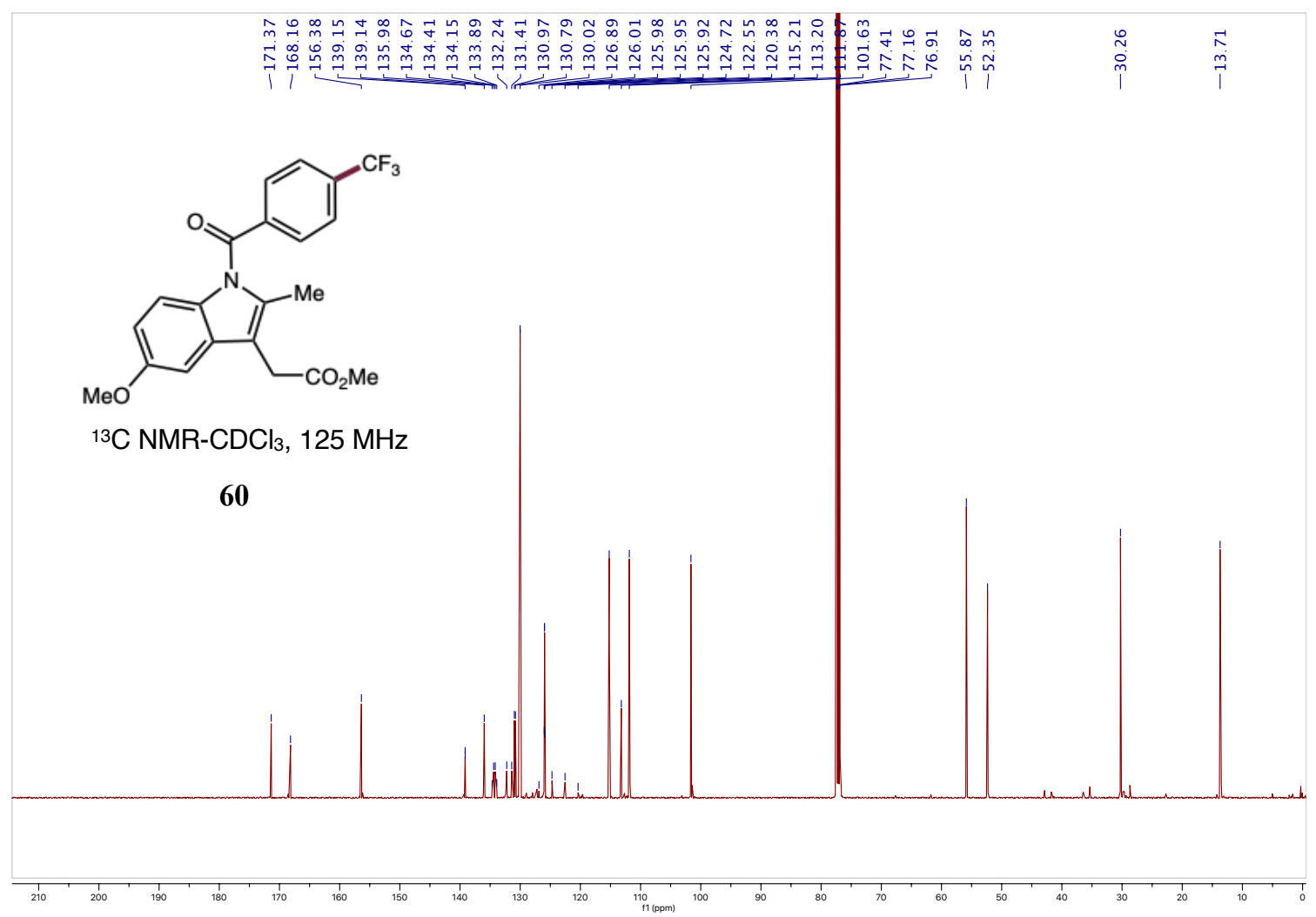

$\tilde{0}$
$\tilde{n}$
1
1

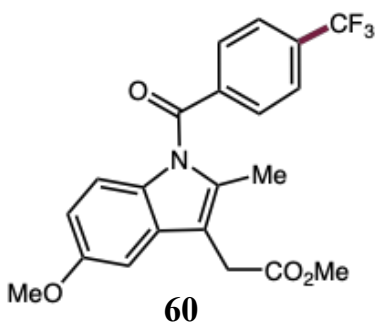

${ }^{19} \mathrm{~F} \mathrm{NMR-CDCl} 3,282 \mathrm{MHz}$

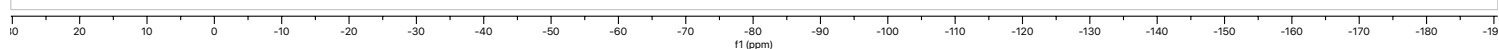




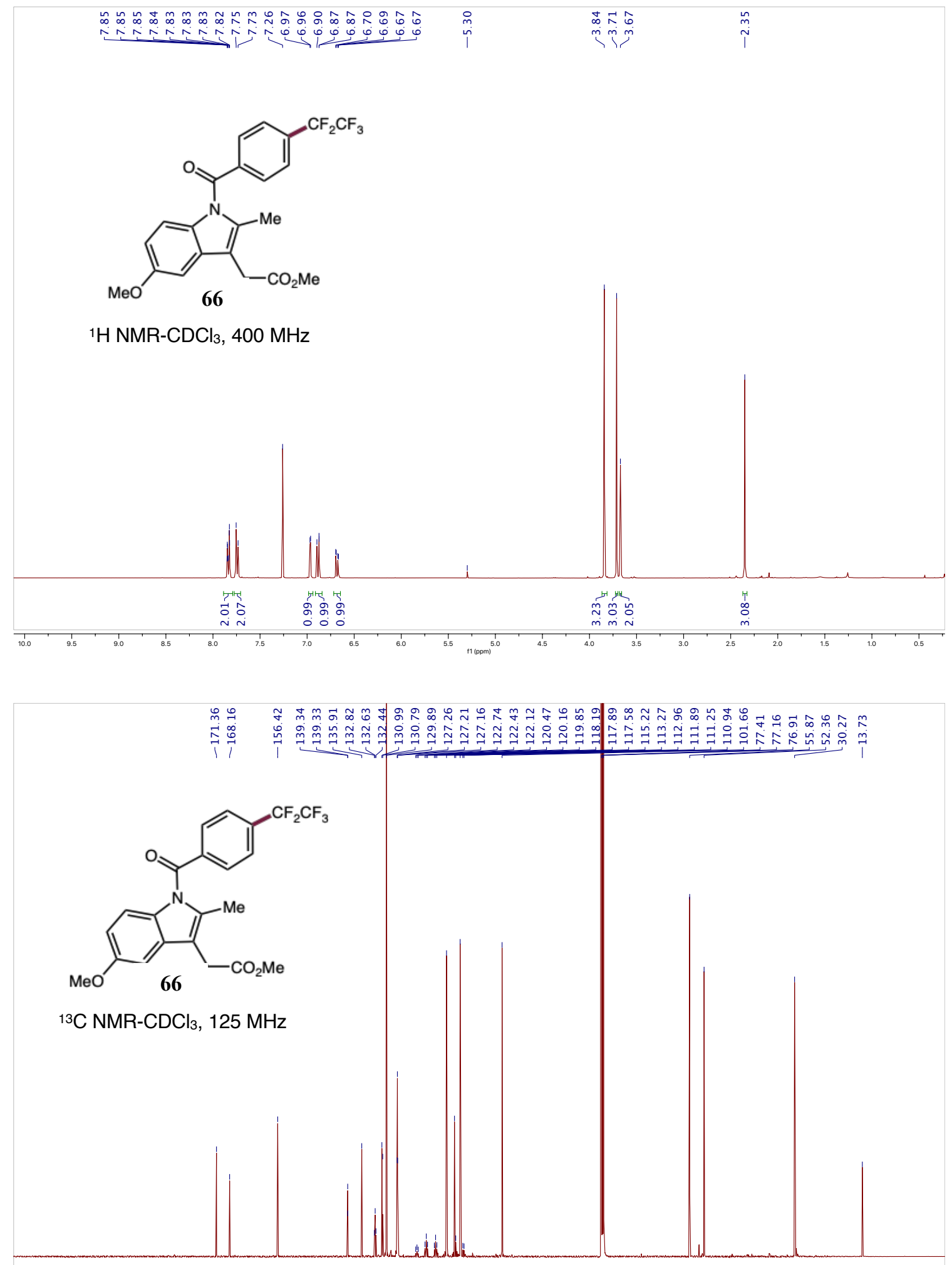




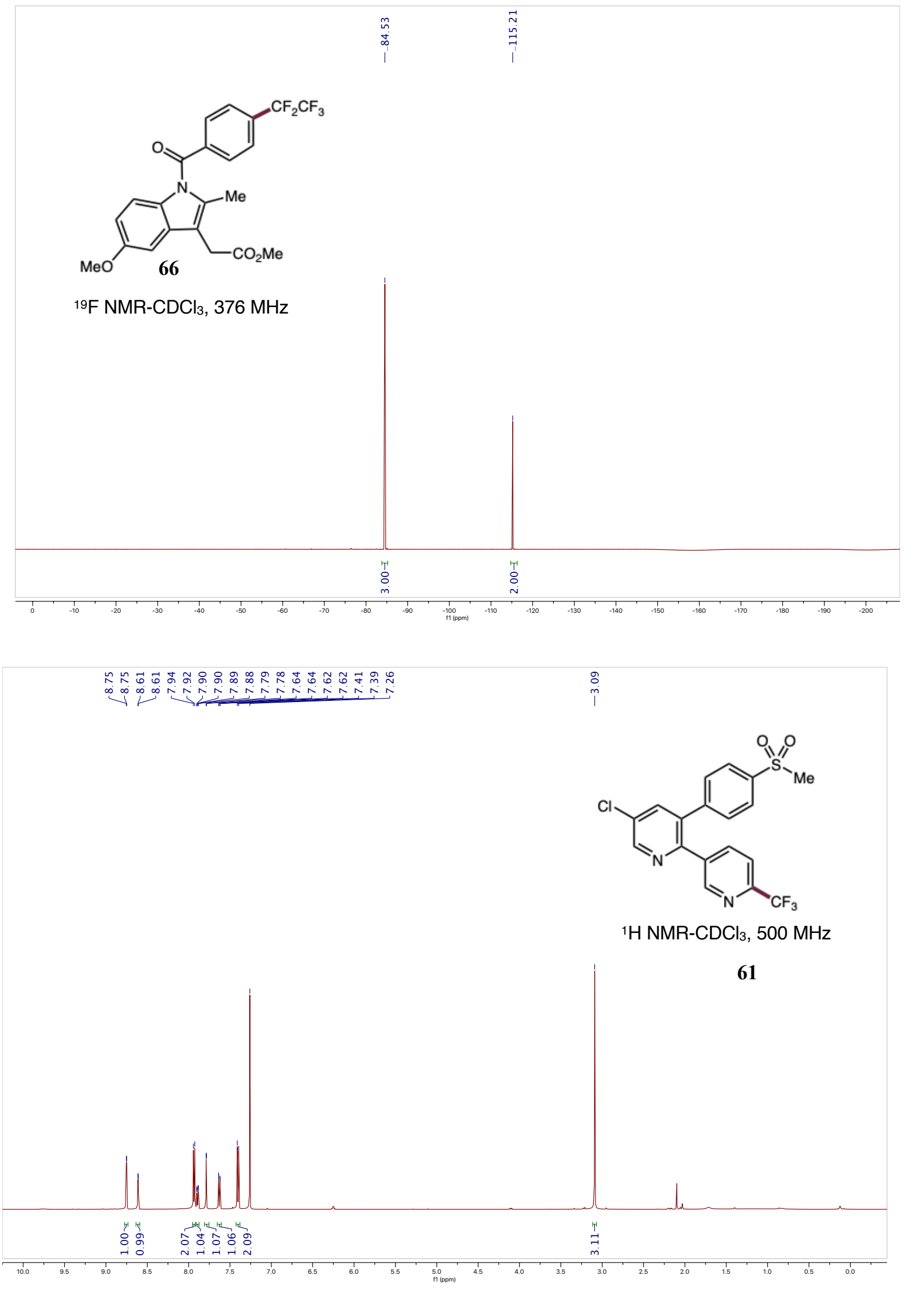



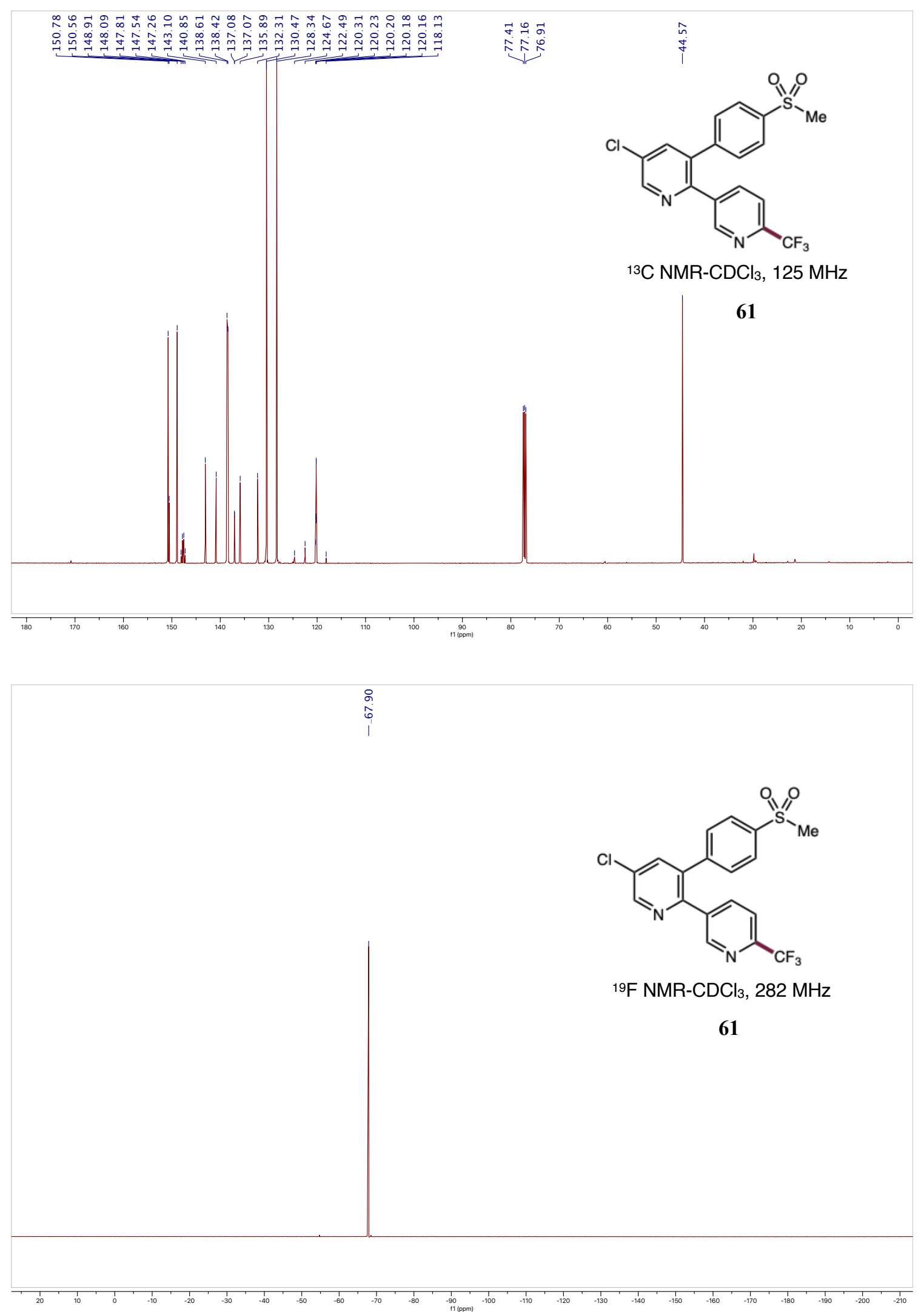


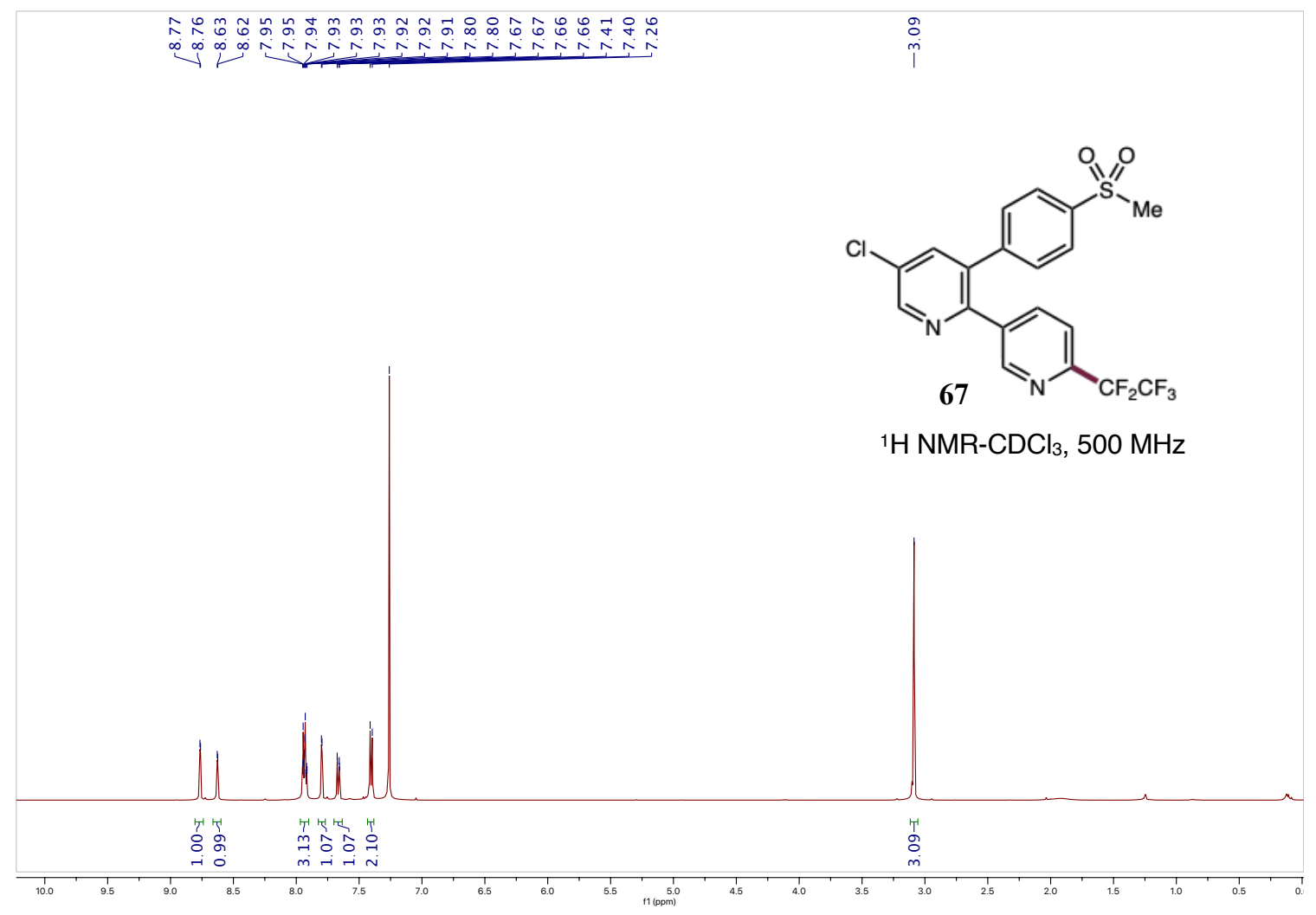

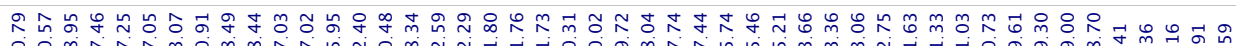

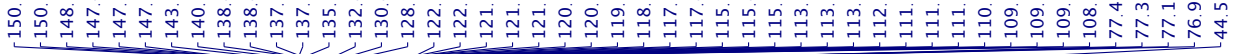

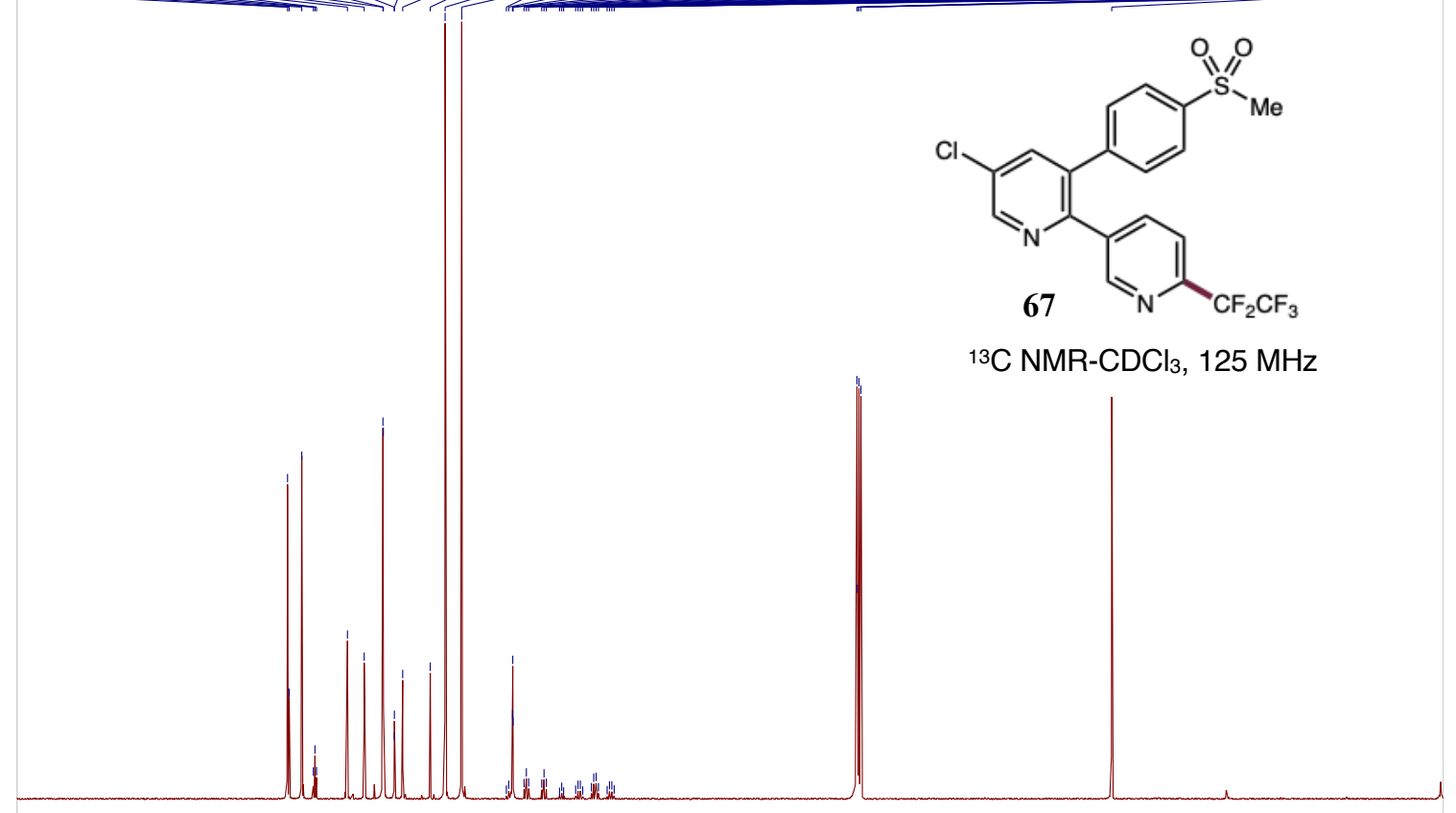



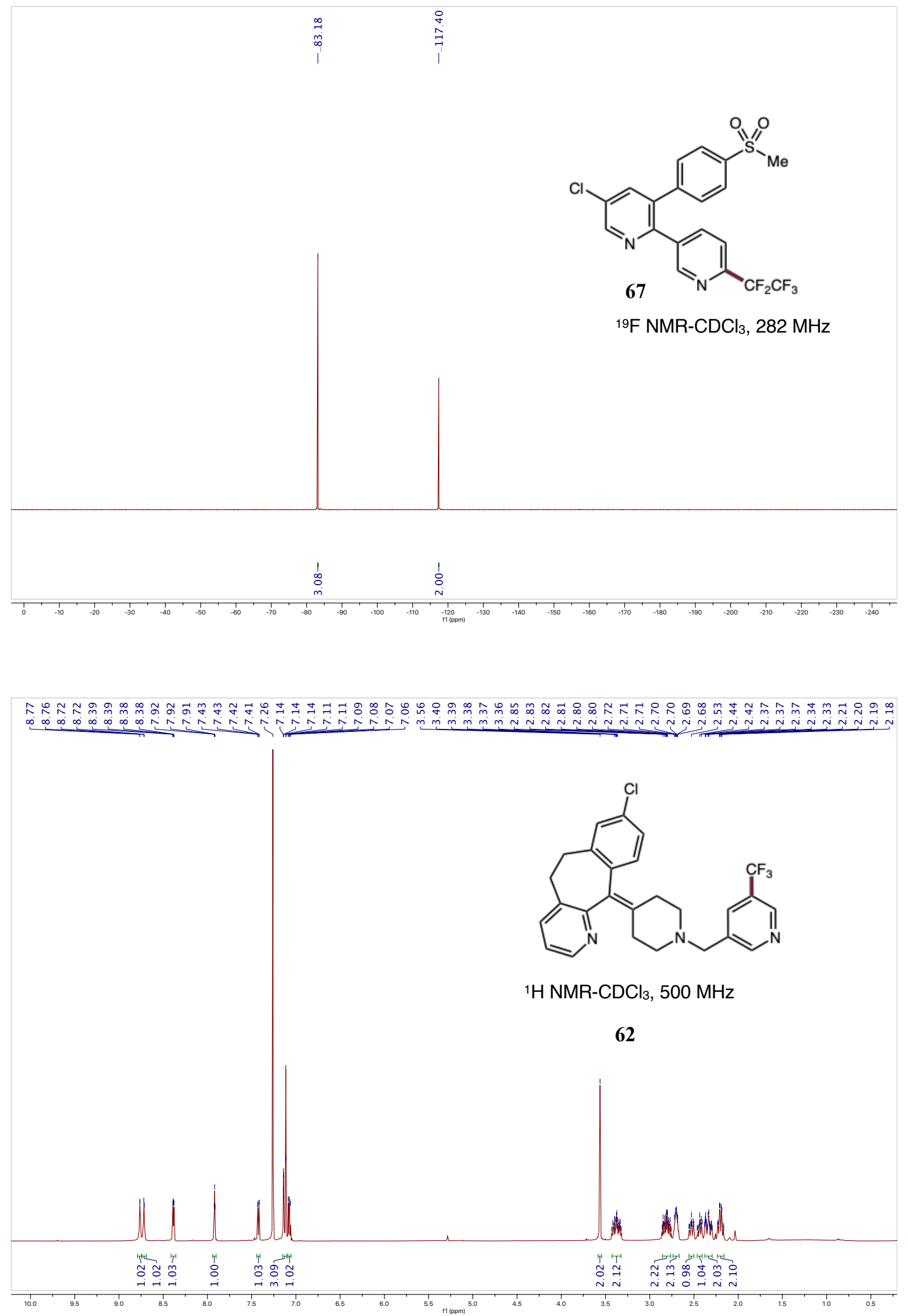

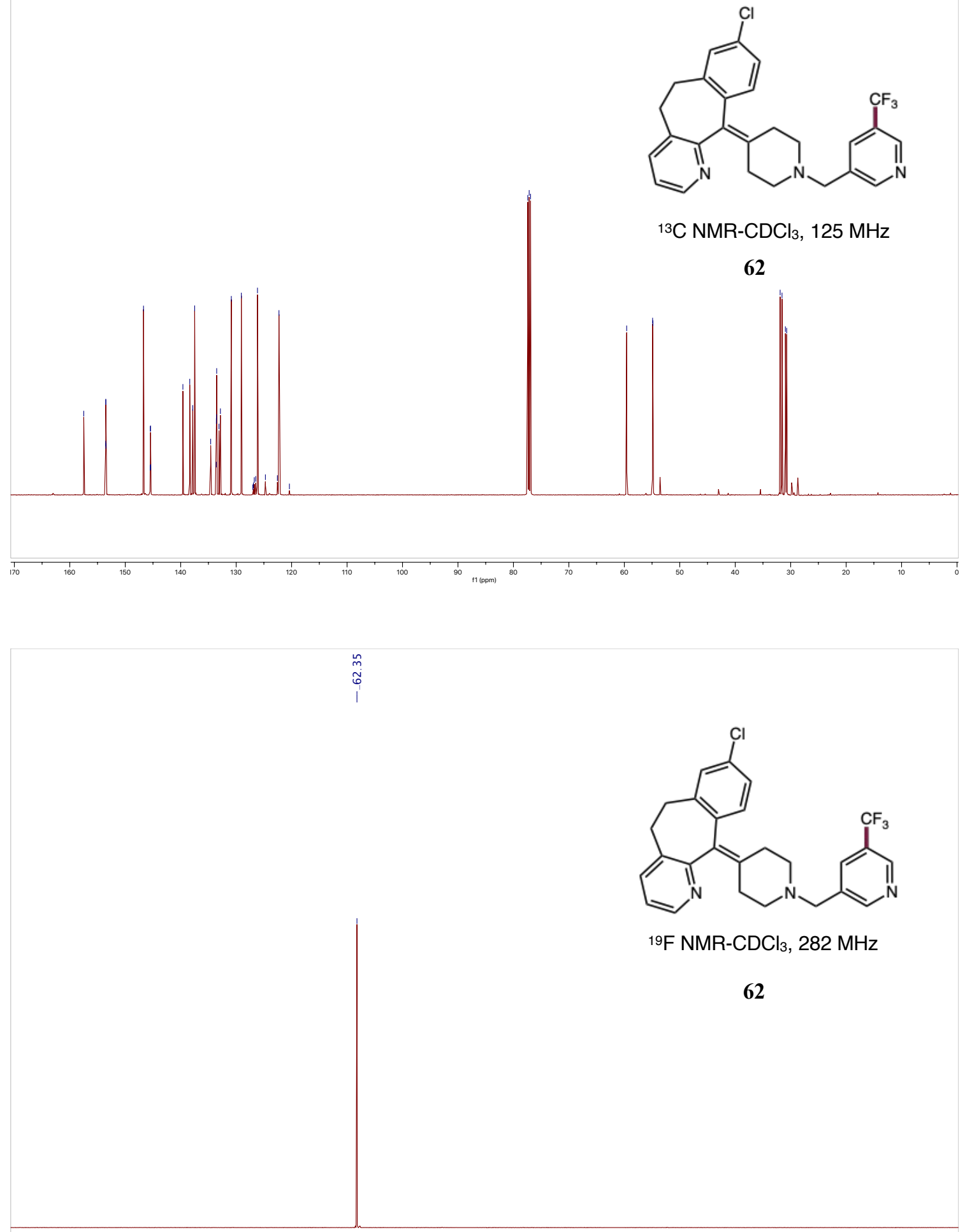


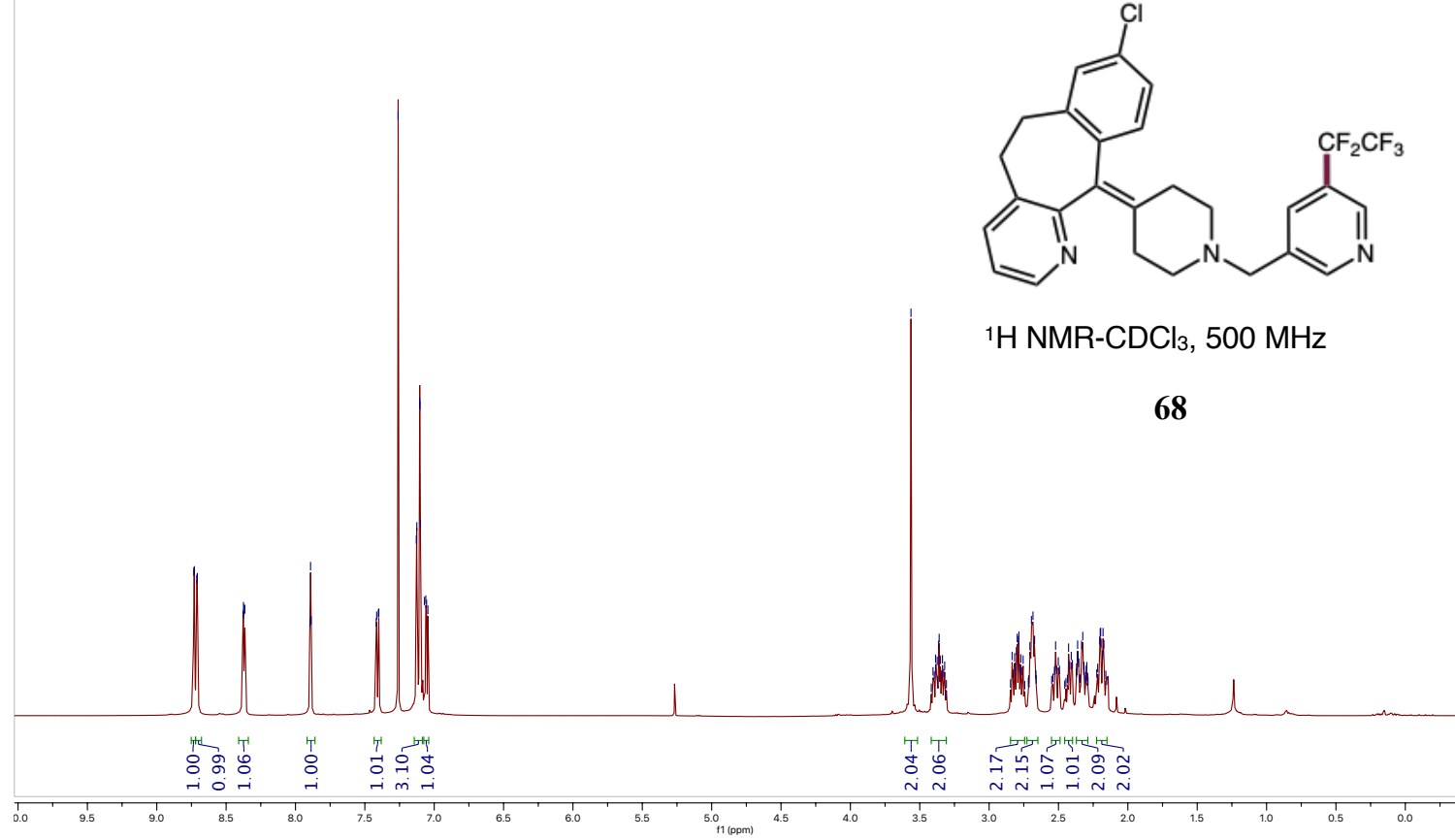

${ }^{1} \mathrm{H} \mathrm{NMR}-\mathrm{CDCl}_{3}, 500 \mathrm{MHz}$

68

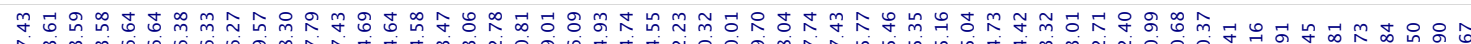

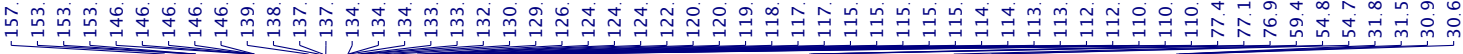
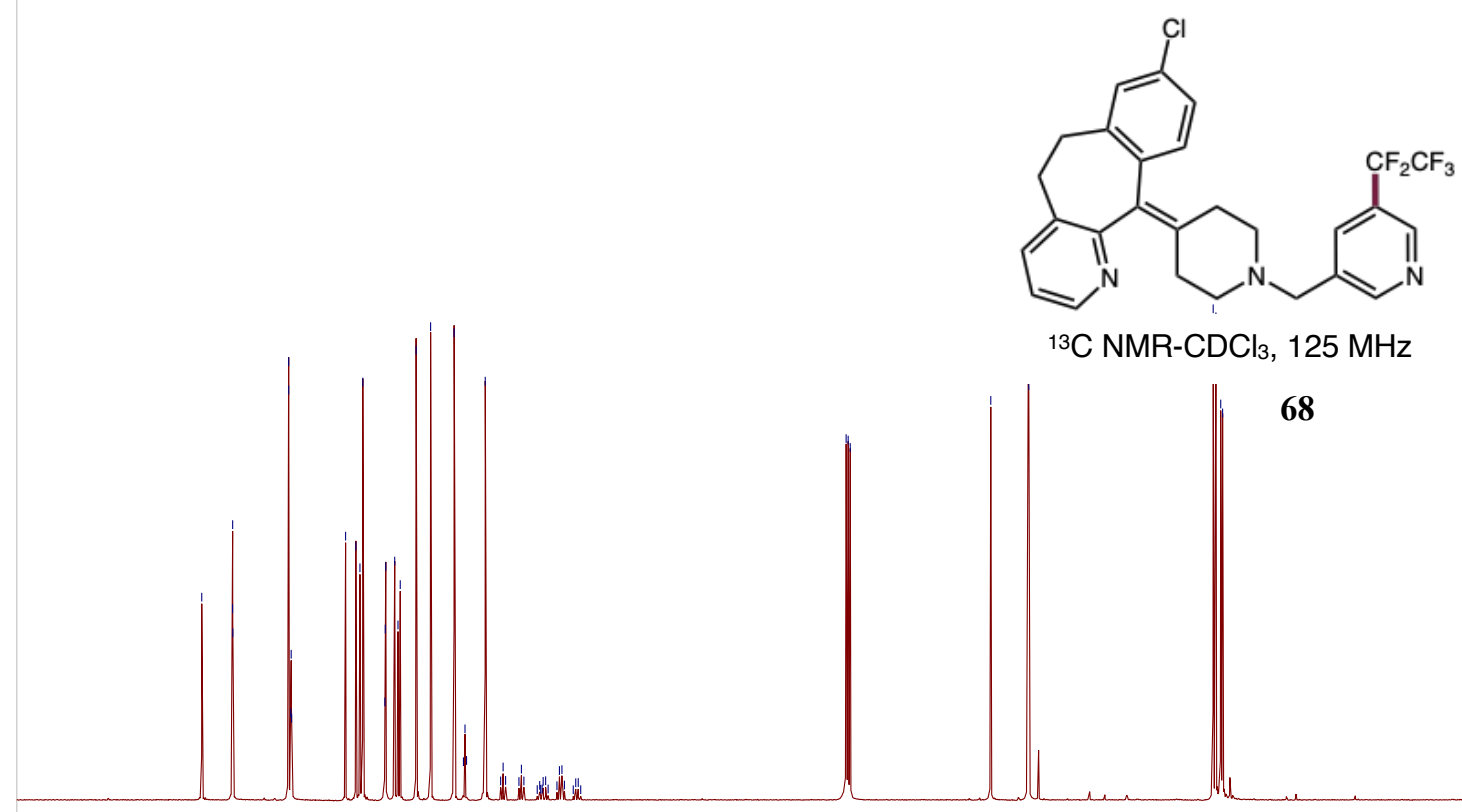

${ }^{13} \mathrm{C} \mathrm{NMR}-\mathrm{CDCl}_{3}, 125 \mathrm{MHz}$

68 


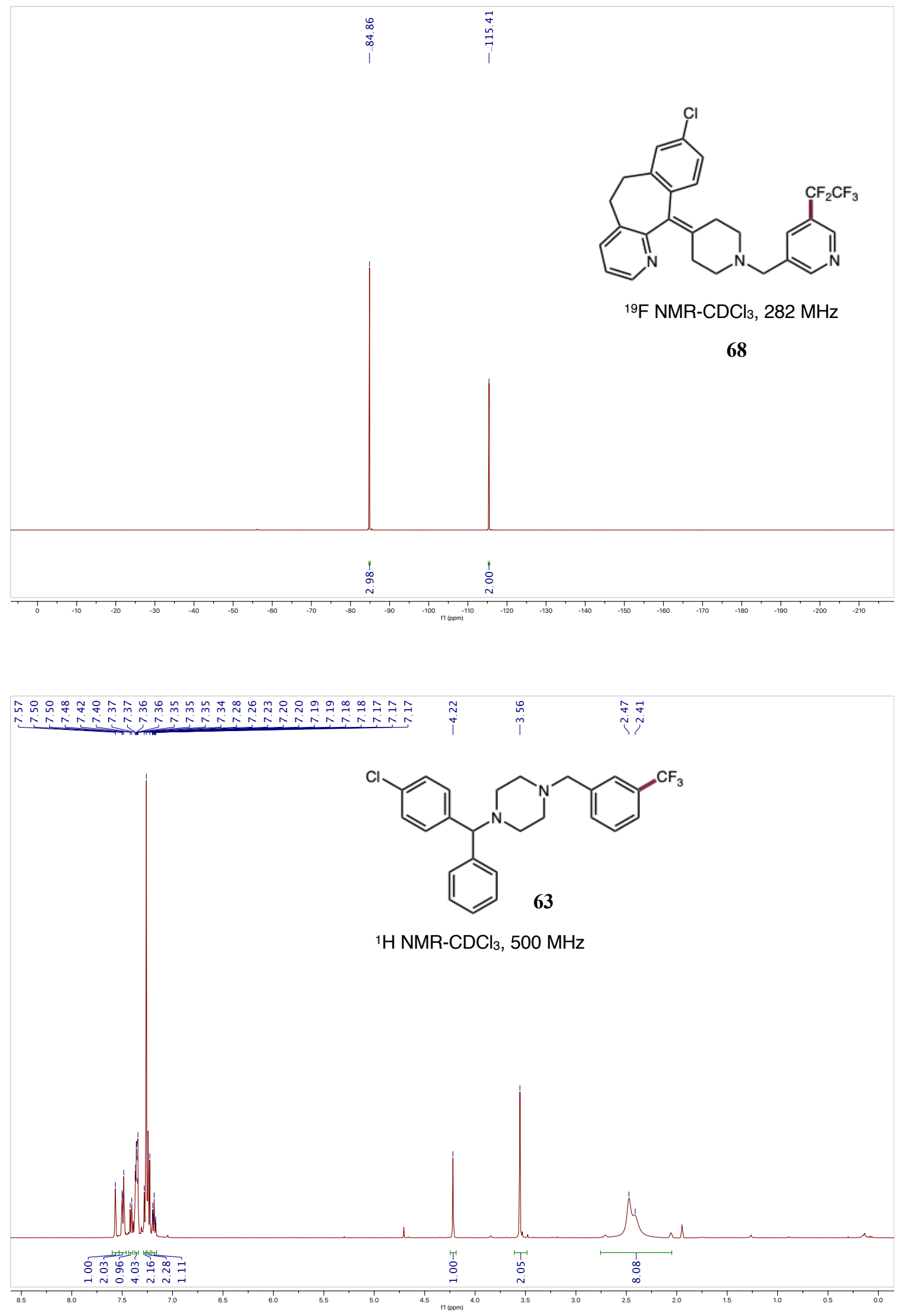




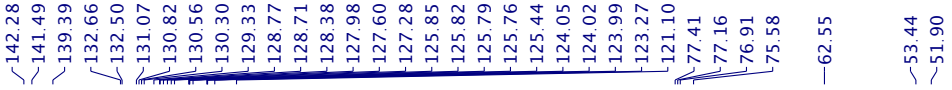
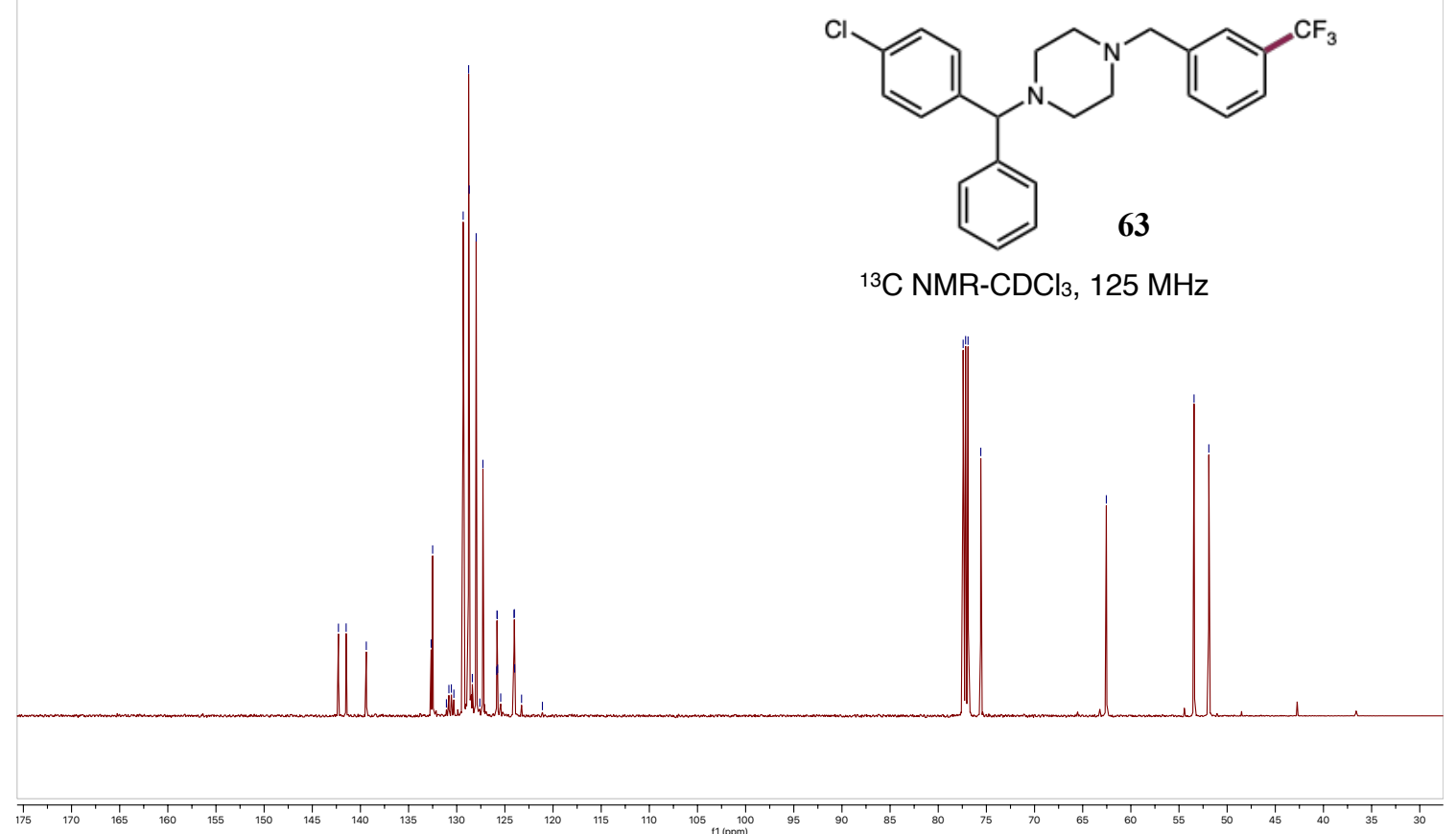

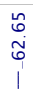<smiles>FC(F)(F)c1cccc(CN2CCN(C(c3ccccc3)c3ccc(Cl)cc3)CC2)c1</smiles>

${ }_{19} \mathrm{~F} \mathrm{NMR-CDCl} 3,470 \mathrm{MHz}$
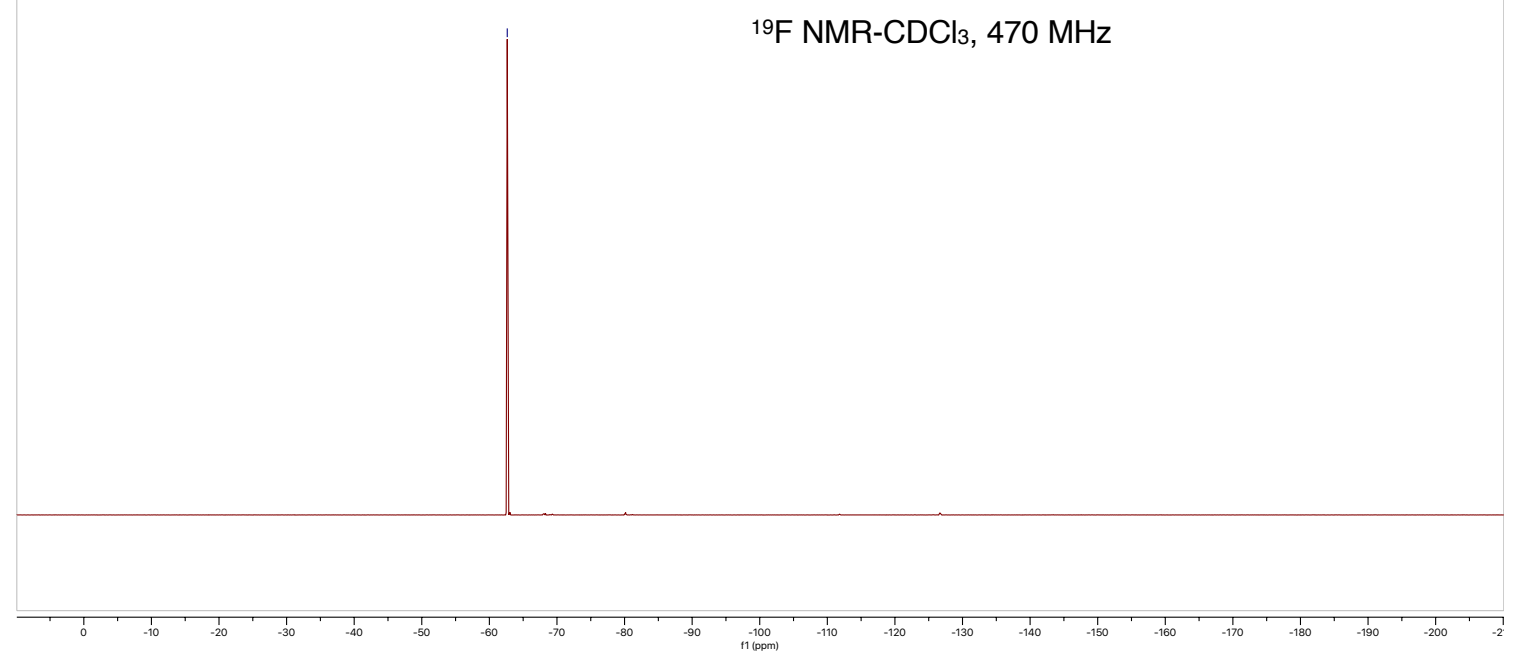

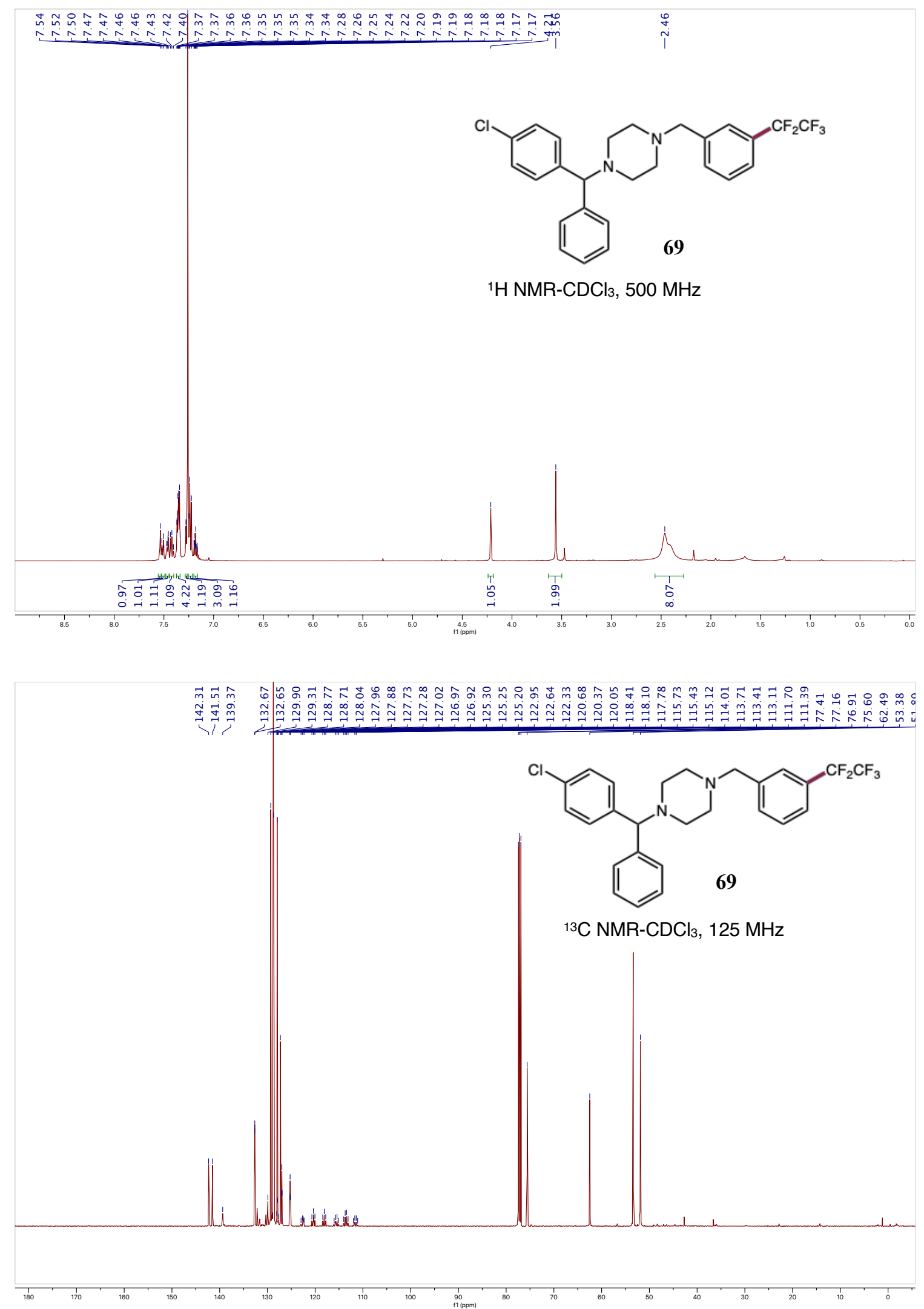


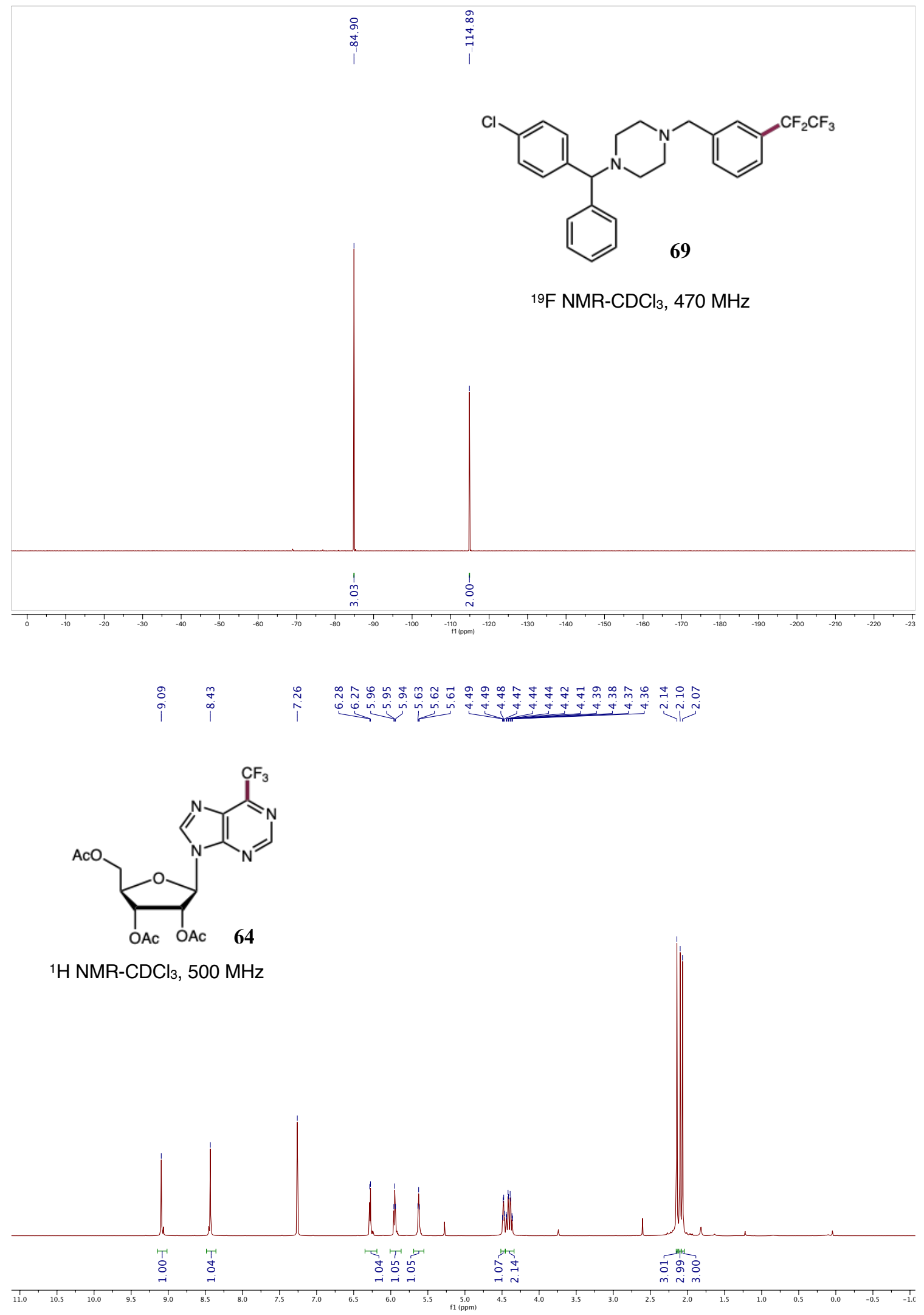




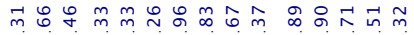

월

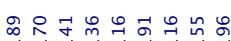

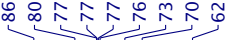

-

$\underbrace{i}$

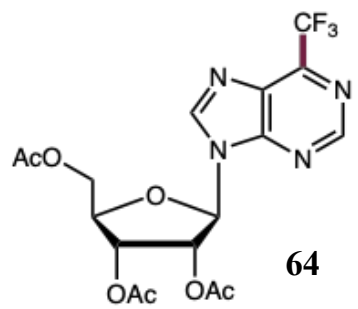

${ }^{13} \mathrm{C} \mathrm{NMR-CDCl} 3,125 \mathrm{MHz}$

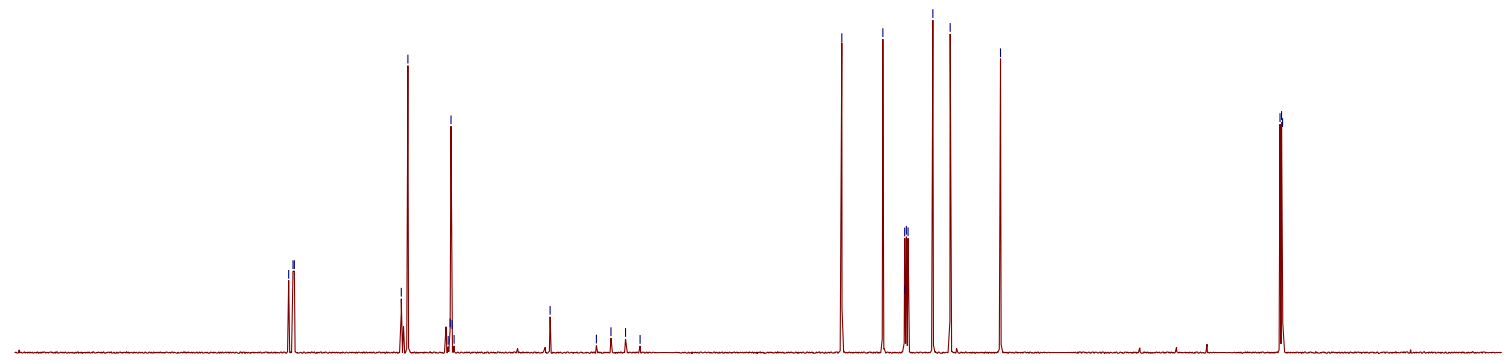

$\underset{210}{1} \quad 200$

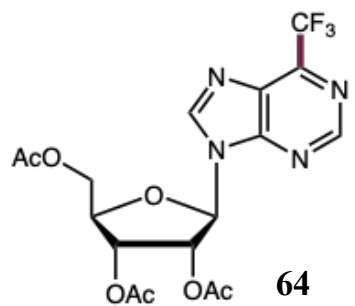

$\sim$
0
0

${ }^{19} \mathrm{~F} \mathrm{NMR-CDCl} 3,470 \mathrm{MHz}$

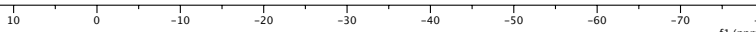



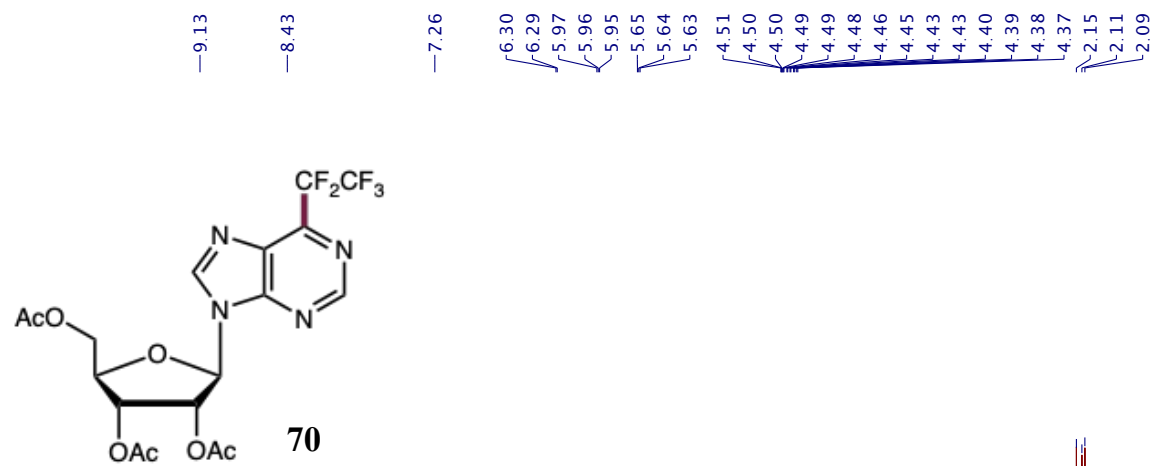

${ }^{1} \mathrm{H} \mathrm{NMR-CDCl}{ }_{3}, 500 \mathrm{MHz}$

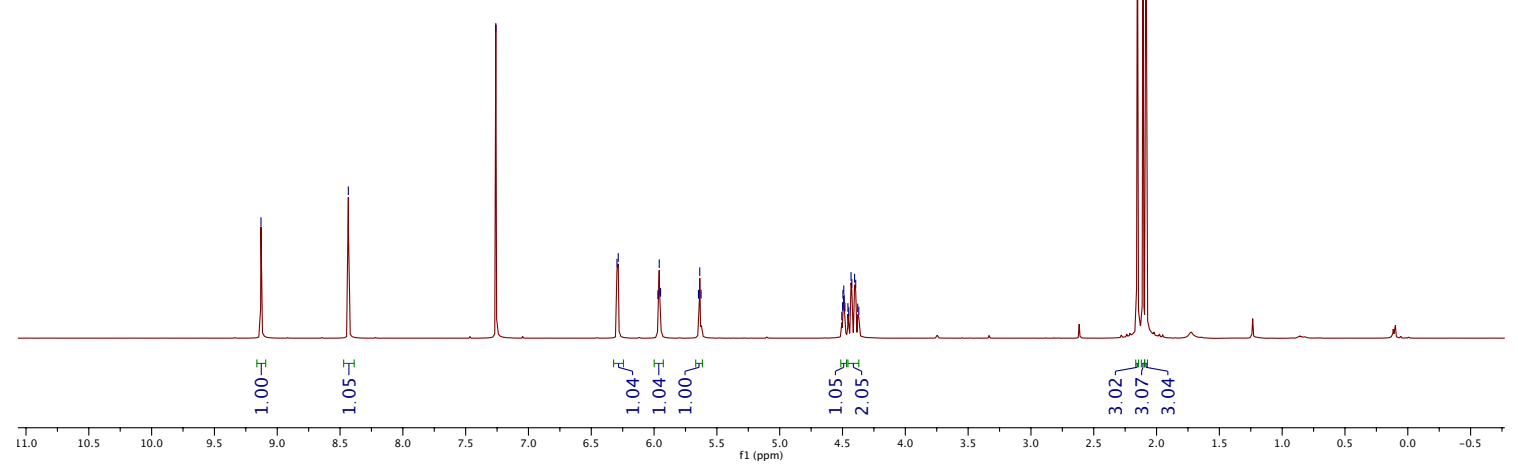

m

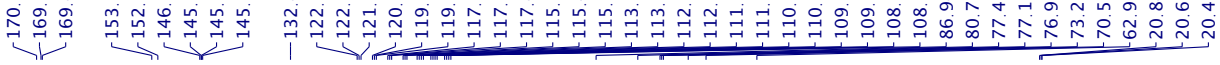

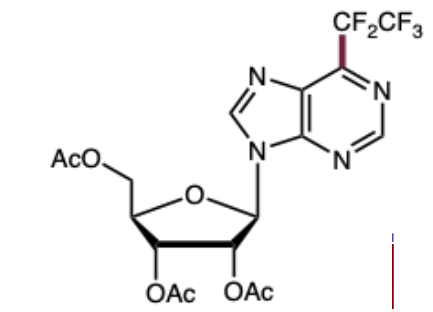

${ }^{13} \mathrm{C} \mathrm{NMR-CDCl} 3,125 \mathrm{MHz}$

70

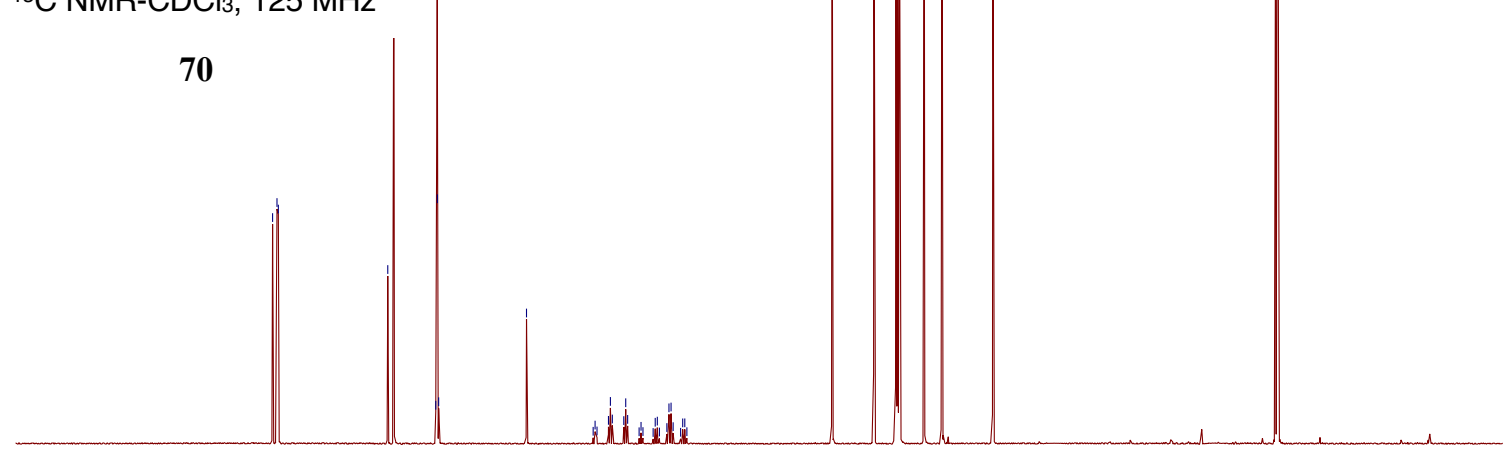




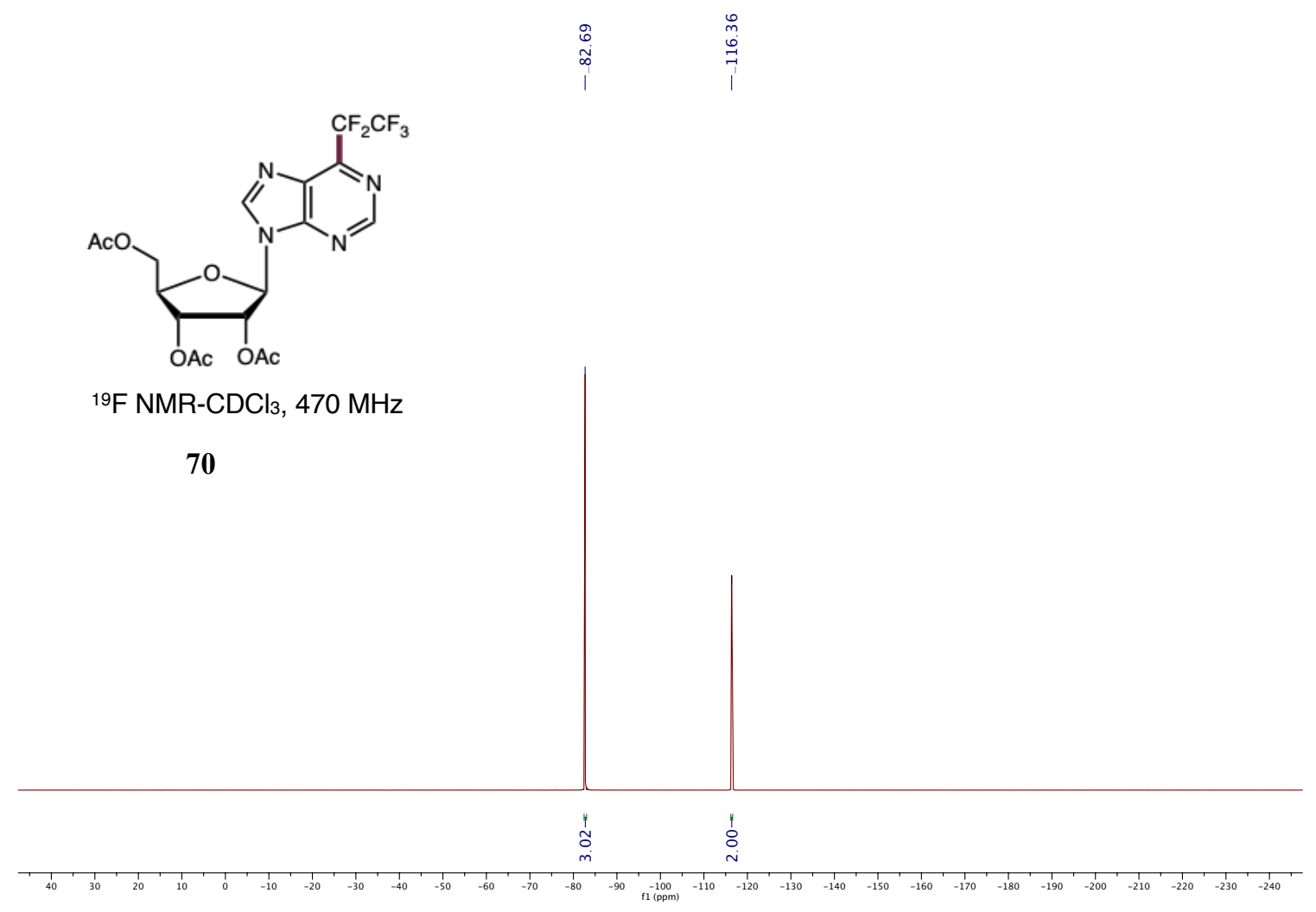




\section{6) References}

1. Pangborn, A. B.; Giardello, M. A.; Grubbs, R. H.; Rosen, R. K.; Timmers, F. J. Safe and Convenient Procedure for Solvent Purification. Organometallics 1996, 15, 1518.

2. Goldsmith, J. I.; Hudson, W. R.; Lowry, M. S.; Anderson, T. H.; Bernhard, S. Discovery and High-Throughput Screening of Heteroleptic Iridium Complexes for Photoinduced Hydrogen Production. J. Am. Chem. Soc. 2005, 127, 7502.

3. Lentz, C.; Schott O.; Auvray, T.; Hanan, Garry.; Elias, B. Photocatalytic Hydrogen Production Using a Red-Absorbing Ir(III)-Co(III) Dyad. Inorg. Chem. 2017, 56, 10875.

4. Sakai, H. A.; Liu, W.; Le, C.; MacMillan, D. W. C. Cross-Electrophile Coupling of Unactivated Alkyl Chlorides. J. Am. Chem. Soc. 2020, 142, 11691.

5. Bacauanu, V.; Cardinal, S.; Yamauchi, M.; Kondo, M.; Fernandez, D. F.; Remy, R.; MacMillan, D. W. C. Metallaphotoredox difluoromethylation of aryl bromides. Angew. Chem. Int., Ed. 2018, $57,12543$.

6. Terrazas, M.; Ariza, X.; Farràs, J.; Yang, J. M. G.; Vilarrasa, J. A direct, efficient method for the preparation of $\mathrm{N}^{6}$-protected ${ }^{15} \mathrm{~N}$-labeled adenosines. J. Org. Chem. 2004, 69, 5473.

7. Le, C. C.; Wismer, M. K.; Shi, Z.-C.; Zhang, R.; Conway, D. V.; Li, G.; Zachal, P.; Davies, I. W.; MacMillan, D. W. C. A general small-scale reactor to enable standardization and acceleration of photocatalytic reactions. ACS Cent. Sci. 2017, 3, 647. 